\title{
Educazione Linguistica.
} Language Education

Vol. 9 - Num. 1

Edizioni Marzo 2020 Ca'Foscari 



\section{EL.LE \\ Educazione Linguistica. Language Education}

Edizioni Ca' Foscari - Digital Publishing Fondazione Università Ca' Foscari Venezia Dorsoduro 3246, 30123 Venezia URL http://edizionicafoscari.unive.it/it/edizioni/riviste/elle/ 


\title{
EL.LE. \\ Educazione linguistica. Language Education
}

\author{
Rivista quadrimestrale
}

Comitato scientifico Simona Bartoli Kucher (UniversitätGraz, Österreich) Antonella Benucci (Università per Stranieri di Siena, Italia) Marilisa Birello (Universitat Autònoma de Barcelona, España) Fabio Caon (Università Ca' Foscari Venezia, Italia) Enrico Cecconi (University of Bath, UK) Carmel M. Coonan (Università Ca' Foscari Venezia, Italia) Diego Cortes (California State University, Long Beach, USA) Bruna Di Sabato (Università degli Studi Suor Orsola Benincasa, Napoli, Italia) Radica Nikodinovska (Methodius University, Skopje, Makedonija) Matteo Santipolo (Università dagli Studi di Padova, Italia) Graziano Serragiotto (Università Ca' Foscari Venezia, Italia) Maria Yashina (Moscow Business University, Russia) Nives Zudič (Univerza na Primorskem, Koper, Slovenija

Comitato di redazione Paolo Balboni (Università Ca' Foscari Venezia, Italia) Carlos Alberto Melero Rodríguez (Università Ca' Foscari Venezia, Italia) Marco Mezzadri (Università di Parma, Italia) Anna Lia Proietto Basar (Yıldız Teknik Üniversitesi, İstanbul, Türkiye) Rita Scotti (Juraj Dobrila University of Pula, Croatia) Enrico Serena (Ruhr-Universität Bochum, Deutschland) Antonio Ventouris (Aristotle University of Thessaloniki, Greece)

Revisori DaliaAbdullah (Ain Shams University, Cairo, Egypt) Andrea Balbo (Università degli Studi di Torino, Italia) Elena Ballarin (Università Ca' Foscari Venezia, Italia) Mirela Boncea (West University of Timisoara, Romania) Elisabetta Bonvino (Università degli Studi Roma Tre, Italia) Giovanna Carloni (Università degli Studi di Urbino «Carlo Bo», Italia) Vanessa Castagna (Università Ca'Foscari Venezia, Italia) Veronique Castellotti (Université «François-Rabelais», Tours, France) Stefania Cavagnoli (Università degli Studi Roma «TorVergata», Italia) Paola Celentin (Università degli Studi diVerona, Italia) Cristiana Cervini (Alma Mater Studiorum Università di Bologna, Italia) Edith Cognigni (Università di Macerata, Italia) Daria Coppola (Università per Stranieri di Perugia, Italia) Elisa Corino (Università degli Studi di Torino, Italia) Michele Daloiso (Università degli Studi di Parma, Italia) Mariapia D’Angelo (Università «G. d'Annunzio» Chieti-Pescara, Italia) Maddalena de Carlo (Università diCassino, Italia) Paolo Della Putta (Università del Piemonte Orientale, Italia) Anna de Marco (Università della Calabria, Italia) Paola Desideri (Università degli Studi «G. D’Annunzio» Chieti Pescara, Italia) Vesna Deželjin (University of Zagreb, Croatia) Pierangela Diadori (Università per Stranieri di Siena, Italia) Emilia Di Martino (Università degli Studi Suor Orsola Benincasa, Napoli, Italia) Giuliana Fiorentino (Università degli Studi del Molise, Italia) Francesca Gallina (Università di Pisa, Italia) Roberta Grassi (Università degli Studi di Bergamo, Italia) Giulia Grosso (Università per Stranieri di Siena, Italia) Amina Hachoulf (Badji Mokhtar University, Annaba, Algeria) Elisabetta Jafrancesco (Università degli Studi di Firenze, Italia) Marie Christine Jamet (Università Ca' Foscari Venezia, Italia) Zorana Kovacevic (University of Banja Luka, Bosnia and Herzegovina) Matteo La Grassa (Università per Stranieri di Siena, Italia) Terry Lamb (University of Westminster, London, UK) Liliana Landolfi (Università degli Studi di Napoli «L'Orientale», Italia) Maslina Ljubicic (University of Zagreb, Croatia) Ivan Lombardi (University of Fukui, Japan) Geraldine Ludbrook (Università Ca' Foscari Venezia, Italia) Maria Cecilia Luise (Università degli Studi di Udine, Italia) Sabrina Machetti (Università per Stranieri di Siena, Italia) Sandra Mardešić (University of Zagreb, Croatia) Carla Marello (Università degli Studi di Torino, Italia) Marcella Maria Mariotti (Università Ca' Foscari Venezia, Italia) Giuseppe Maugeri (Università Ca' Foscari Venezia, Italia) Patrizia Mazzotta (Università degli Studi di Bari «Aldo Moro», Italia) Marcella Menegale (Università Ca' Foscari Venezia, Italia) Darja Mertelj (University of Ljubljana, Slovenia) Nikita Mihaljevic (University of Split, Croatia) Eliana Moscarda Mirković (Juraj Dobrila University of Pula, Croatia) Anthony Mollica (Brock University, St. Catharines, Ontario, Canada) Johanna Monti (Università degli Studi di Napoli «L'Orientale», Italia) David Newbold (Università Ca' Foscari Venezia, Italia) Paolo Nitti (Università degli Studi dell'Insubria, Italia) Alberta Novello (Università degli Studi di Padova, Italia) Cristina Onesti (Università degli Studi di Torino, Italia) Gabriele Pallotti (Università degli Studi di Modena e Reggio Emilia, Italia) Elisabetta Pavan (Università degli Studi di Padova, Italia) Ruggiero Pergola (Università degli Studi di Bari «Aldo Moro», Italia) Gianfranco Porcelli (Università Cattolica del Sacro Cuore, Milano, Italia) Rosa Pugliese (Alma Mater Studiorum Università di Bologna, Italia) Simonetta Puleio (Universität Stuttgart, Deutschland) Federica Ricci Garotti (Università degli Studi di Trento, Italia) Fabio Ripamonti (University of South Bohemia in České Budějovice, Czech Republic) Flora Sisti (Università degli Studi di Urbino «Carlo Bo», Italia) Camilla Spaliviero (Università Ca' Foscari Venezia, Italia) Antonio Taglialatela (Università degli Studi della Tuscia, Viterbo, Italia) Giulia Tardi (Università degli Studi di Firenze, Italia) Simone Torsani (Università degli Studi di Genova, Italia) Victoriya Trubnikova (Università degli Studi di Padova, Italia) Ada Valentini (Università degli Studi di Bergamo, Italia)

Direttore responsabile Paolo Balboni

Redazione | Head office Università Ca' Foscari Venezia| Dipartimento di Studi Linguistici e Culturali Comparati|Centro di Ricerca sulla Didattica delle Lingue | Ca' Bembo | Dorsoduro 1075 - 30123 Venezia, Italia | elle@unive.it

Editore Edizioni Ca' Foscari|Fondazione Università Ca' Foscari Venezia| Dorsoduro 3246, 30123 Venezia, Italia|ecf@unive.it

(c) 2020 Università Ca' Foscari Venezia

(c) 2020 Edizioni Ca' Foscari - Digital Publishing per la presente edizione

\section{(c) (1)}

Quest'opera è distribuita con Licenza Creative Commons Attribuzione 4.0 Internazionale This work is licensed under a Creative Commons Attribution 4.0 International License

Certificazione scientifica delle Opere pubblicate da Edizioni Ca' Foscari - Digital Publishing: tutti i saggi pubblicati hanno ottenuto il parere favorevole da parte di valutatori esperti della materia, attraverso un processo di revisione anonima sotto la responsabilità del Comitato scientifico della rivista. La valutazione è stata condotta in aderenza ai criteri scientifici ed editoriali di Edizioni Ca' Foscari.

Scientific certification of the works published by Edizioni Ca' Foscari - Digital Publishing: all articles published in this issue have received a favourable opinion by subject-matter experts, through an anonymous peer review process under the responsibility of the Scientific Committee of the journal. The evaluations were conducted in adherence to the scientific and editorial criteria established by Edizioni Ca' Foscari. 
Vol. 9 - Num. 1 - Marzo 2020

\section{Sommario}

\section{A Non-Culture-Bound Theory of Language Education}

Didattica dell'italiano L2 ad apprendenti adulti analfabeti Uno studio di caso all'interno di CPIA, SPRAR e CAS della Provincia di Venezia

Annalisa Brichese, Camilla Spaliviero, Valeria Tonioli

Apprendimento dell'italiano in Camerun

Comprendere e usare le espressioni idiomatiche e altre locuzioni fisse

The Use of the Verb Run in English Learner-Narratives An Analysis of Verb Constructions Influenced by Different L1s Martina Irsara, Ulrike Domahs

Strategie motivazionali per la classe di lingue Rassegna e confronto delle ricerche empiriche sugli apprendenti Giacomo Cucinotta

Canzoni per l'insegnamento linguistico-culturale del tedesco e per la didattica della traduzione

Variabilità fonetico-sintattica nella Commercial Contemporary Music come oggetto di studio per le classi avanzate di inglese come L2

Lo sviluppo della competenza traduttiva e l'uso della traduzione nella didattica delle lingue moderne 



\section{EL.LE}

Vol. 9 - Num. 1 - Marzo 2020

\section{A Non-Culture-Bound Theory of Language Education}

Paolo Balboni

Università Ca’ Foscari Venezia, Italia

Abstract Educational linguistics is a dyadic science. The noun, linguistics, is a broad term which includes neuro-, psycho-, socio-, pragma-, ethno-linguistics and communication studies: areas where national 'schools' non longer exist. Educational, on the contrary, is a culture-bound term: language teaching is carried out according to laws which concern syllabi, exams and certifications, the language(s) of instruction, the teaching of the host language to migrant students, teacher training programmes etc. These juridical and administrative acts are meant for the local educational systems. We propose that it is possible to find a number of principles and models (we call them "hypotheses") which can be accepted by culture-bound educational decision-makers, thus increasing consistency within language teaching and research throughout the world.

Keywords Educational linguistics. Edulinguistics. Language education. Language teaching. Communicative competence. Intercultural communication. Semiotic competence.

Summary 1 Educational Linguistics, a Dyadic Science. - 2 A Theory of Language Education Based on 'Models'. - 3 A Theoretical Framework Based on Eight 'Models'or 'Hypotheses'. 


\section{Educational Linguistics, a Dyadic Science}

Educational linguistics, edulinguistics, applied linguistics, language pedagogy, didactologie des langues-cultures, glottodydaktica, Sprachunterricht, and so on are dyadic names for a dyadic science. From the educational linguists' point of view, the dyad is "LANGUAGE + education", while decision-makers and authorities in educational systems view it as "EDUCATION + language". The opposition can be defined as Educational linguistics vs. Language pedagogy.

Since the 1990s, epistemological research on the nature of edulinguistics has flourished. The noun, linguistics, has become a broad term which includes neuro-, psycho-, socio-, pragma-, ethno-linguistics as well as communication studies. ${ }^{1}$ Conversely, the adjective, educational, still remains rather vague and indistinct in meaning. Bibliographies are compiled from essays and books written by scholars like the Author of the present article who come from the field of linguistics. As a consequence, linguistics (in the broader sense indicated above) has been a transnational and transcultural science for the last century. This has resulted in a widespread consistency among American, European, Asian and African edulinguistic research. Differences exist, of course, but they are mostly philosophical (innatism vs. emergentism; form vs. use; lexicalized grammar vs. grammaticalized lexis, and so on) rather than culture-bound and based on national traditions.

However, if we move from language education research to actual language teaching, the situation is quite different. In fact, teaching is carried out according to laws, acts and decrees which not only concern native and non-native language syllabi but also the language(s) of instruction, the teaching of the host language to migrant students, exams and certifications, initial and in-service training programmes for language teachers, and so on. These juridical and administrative acts are meant for the national or local educational systems and, as such, are strictly culture-bound.

We propose that it is possible to find a number of principles, which we call 'hypotheses', that can be accepted not only by globalized edulinguists, but by culture-bound educational decision-makers as well, thus increasing consistency in language teaching research and practice in the world. In order to support this thesis, a theory of language education and teaching is needed (a deeper analysis of the problem is in Balboni 2018, which is the basis of this essay).

1 A general idea of the range of contents of educational linguistics can be found in fundamental books such as Clapham 1997; Spolsky, Hult 2007; Hornberger 2008; Chapelle 2012; Balboni 2018. 


\section{A Theory of Language Education Based on 'Models'}

A theory aims at providing a reference paradigm that is considered true until a paradigm shift occurs. If a non-culture-bound paradigm exists, as we think it does, each culture-bound educational system has the possibility of checking its traditional way of teaching native and non-native languages against the 'true' principles of the framework. This, in turn, initiates a process of increasing consistency between local traditions and the universal framework.

In order to be considered true, a paradigm has to be built on reliable 'hypotheses', that is, on statements that are hypo, 'below', the thesis. If hypotheses are wrong or even highly questionable, the main thesis, the paradigm, is not reliable.

In order to produce true hypotheses - 'true' according to current criteria of verifiability and falsifiability - we have chosen logical models as our main instrument.

In fact, the history of scientific research is the history of the quest for true knowledge. Many instruments have been used to this end such as theorems, which are based on empirical verification (it is possible to check with a metre that in a right triangle the squares built on the catheti have the same area as the square built on the hypothenuse); syllogisms, which are based on a strict formal structure ("men $[\mathrm{A}]$ are mortal [B]; Socrates [C] is a man [A]; thus Socrates [C] is mortal $[\mathrm{A}]$ ": $\mathrm{A}=\mathrm{B} ; \mathrm{C}=\mathrm{A}$; thus $\mathrm{C}=\mathrm{B}$ ); and the laws of physics, of phonetics, of chemistry and so on, which are usually considered true on the basis of empirical experience. Yet, Einstein reminded the world of science that empirical evidence might be misleading (in 1915 Einstein claimed that time was not a constant datum - which was empirically demonstrated only a century later).

Einstein opened the way to a post-Galilean way of conceiving scientific research. According to him, the traditional way (observing, making hypotheses, and then verifying them) was weak as human senses are not always reliable when they observe a phenomenon (for example, we all see the sun circling the earth). A reliable truth, on the other hand, is free from empirical verification as it is valid in itself. This is because its logical form is valid. It is true per se, as algebraic equations and chemical formulae are. They are logical models.

The Theory of Models is linked to Alfred Tarsky who felt language is an inadequate instrument to be employed in the search for truth. This is because language is ambiguous, changes over time and space, and is too open to interpretation. In fact, from the 1950s onwards, Tarsky became more and more interested in mathematics, a path that Wittgenstein had anticipated some decades earlier, and models, as a result, have become increasingly numerical and diagrammatic.

Theorems and syllogisms deal with very small sections of truth and are defined by the interaction of very few factors and statements. 
Models, as we use this term, describe events that are more complex than theorems and syllogisms. Further, they use language systematically in their description alongside numbers, graphic symbols and diagrams. Yet, language use is limited to the two types of statements defined by cognitive psychology: declarations, simple sentences usually based on verbs such as be, be made of, and have, which are very easy to verify or falsify; and procedures, based on the if... then... sequence, where if introduces one or more declarations and then introduces the consequence.

Our definition of 'model' is based on a series of declarations (see Balboni 2011a, 2011b), but only two fundamental declarations are considered in this essay, that is:

a. models are universal, i.e., they describe all possible occurrences of a phenomenon or of a notion.

For instance, the model of communicative competence indicated in Hypotheses 5 and 6 below claims that it describes communicative competence in all cultures and languages, anytime and anywhere. It may be used in a non-culture-bound syllabus only if it satisfies this claim; and,

b. models can be simple or complex.

Simple models are forms of declarative knowledge, based on a topic, a copula and the core of the statement ("verbs are a class of words that function as the main elements of predicates"). Simple models work on a single level: the Pythagorean theorem, for instance, does not require further models to explain the relation among the squares of its sides.

Complex models, on the other hand, are forms of procedural knowledge. They link statements via an if... then... mechanism ("if languages are made up of different varieties, then a syllabus must indicate which varieties to present and when"). Further, complex models are hierarchical and work on different levels. For example, the model of communicative competence includes other models (of language competence, of comprehension, etc.) which work as secondary or tertiary level models.

Language, including the language used in the two declarations above, may be ambiguous. Ambiguity, both in writing and in comprehending a statement, can be reduced by the use of graphs and diagrams, as discussed in Allwein and Barwise's Logical Reasoning with Diagrams (1996; see also Jonhson-Laird 2002; Holyoak, Morrison 2012; further reading about the Model Theory can be found in Rothmaler 2000). 


\section{A Theoretical Framework Based on Eight 'Models' or 'Hypotheses'}

We claim that the eight models outlined below can be internationally accepted and, that is, there are no cultures or traditions that can deem these models alien to their nature. A slightly different and more in-depth description of the models and the framework's underlying hypo-theses can be found in Balboni 2018.

\section{Hypothesis 1. Logical Models Are a Non-Culture-Bound Way of Finding Truth}

This framework is based on a number of models which are assumed to be right until alternative models are suggested. The nature of the models is outlined in section 2 above.

\section{Hypothesis 2. A Non-Culture-Bound Definition of Language Education}

The whole framework pivots on the following logical graph:

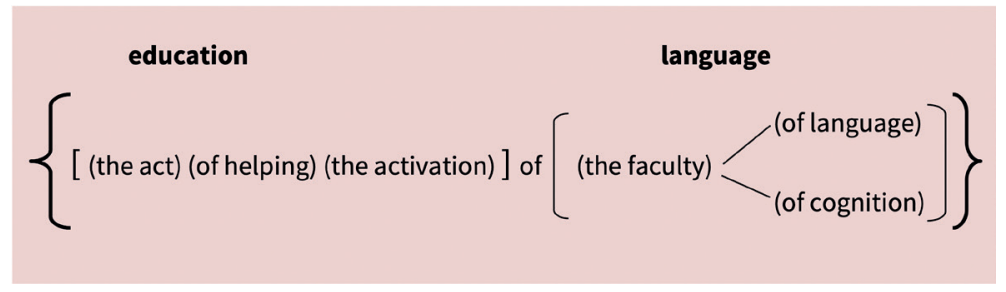

Figure 1 The graph of language education

The graph can be summarized in Alexander Von Humboldt's words: "A language cannot be taught. One can only create conditions for learning to take place". According to the model, language education and, consequently, language teaching are seen as

a. helping the educational system (school systems, curriculum and syllabus designers, textbook authors, teachers, language assistants) provide for

b. the person, however old, who is genetically equipped with the faculty to acquire both native and non-native languages (a definition of 'Faculty of language' can be found in Chomsky, Hauser, Fitch 2002; 'Faculty of cognition', instead, refers to cognitive linguistics). This person, or learner (a child in his 
or her mother's arms, a student, a migrant worker, etc.) is the subject of language teaching, the one who learns a language with the help of others who know the target language and assist his or her acquisition.

The graph, Von Humboldt's principle and the items ' $a$ ' and ' $b$ ' above all concern human nature and human beings, independently from the cultural contexts they are born or grow up in. Language education is implemented in two different language teaching contexts, i.e. (1) native and almost-native language teaching and (2) non-native language teaching:

a. Native and almost-native language teaching:

- L1, the mother tongue: this is not just the mother's tongue, it is the language or languages of the home environment in which the child grows, thus becoming the language(s) of thought (a definition of 'native speaker' can be found in Davies 2003). Teaching the mother language aims at systemizing and improving the quality of a language which, when speakers start their formal education, has already been spontaneously acquired in its oral form. An International Mother Tongue Education Network is growing to support the use of mother tongue(s) in multilingual contexts and in schools where English as a Lingua Franca is used as the language of instruction;

- L2, second language; L2 does not refer to the second language learnt by a person, but to a language present in the environment where it is taught, the first language being the person's native language. Some examples are Catalan for Spaniards and Spanish for Catalans in Barcelona, and French for foreign students in Paris. The student is immersed in the L2 alongside the mother tongue. This means that $\mathrm{L} 2$ teachers have no control of what and how much their students acquire spontaneously (sometimes with errors) in daily life. When L2 proficiency becomes high, let's say B2 according to the CEFR, the nature of L1 and L2 teaching is very similar, i.e. it concerns systemizing and improving the quality of the language rather than acquiring it;

- Ethnic language: this is a peculiar form of L2, that is, a language spoken in a person's community of origin who has not acquired it as a mother tongue but who nonetheless hears it spoken in the family and community environment. For example, the children of immigrants often grow up as host language speakers, yet they hear the ethnic language spoken at home or in radio or TV programmes. They spontaneously develop receptive skills, and sometimes practice speaking when they visit relations during family holidays. Sometimes ethnic 
or community languages are called heritage languages (definitions of 'ethnic language' can be found in Van Deusen-Scholl 2003 and in Brinton, Kagan, Bauckus 2008).

Ethnic and second language teaching contexts are similar in that both these languages are spoken in social contexts. However, the spontaneously acquired ethnic language is often an obsolete or local variety. Thus, when students decide to take a course in order to perfect their ethnic language, what they have picked up, not being representative of the standard variety, may be more of a hindrance than a help;

- Language of instruction: these are used in multilingual countries, as is the case of English in India or French in Maghreb, and in private schools where a non-native language, usually English, is used. It is apparent that the choice of non-native languages as the medium of instruction is a political issue. Many Mother Tongue Education Movements around the world claim that most children in the world receive instruction in a non-native language which hinders effective learning. On the other hand, there is a strong CLIL movement, especially in Europe, which supports the idea that content and language integrated learning enhances the acquisition of the language and does not hinder the acquisition of content.

We shall deal with the role of language as the main instrument of instruction in Hypothesis 2 (see Vollmer 2006a and 2006b).

b. Non-native language teaching

While the objective in group ' $a$ ' is to perfect and systemize a language which has already been acquired spontaneously although in differing degrees, this group includes contexts where the objective is to acquire a non-native language from scratch - or almost from scratch, as in the case of lingua francas used in mass and social media.

Non-native language teaching concerns:

- FL, foreign language: the objective of FL teaching is not to perfect an acquired language, but to start the acquisition process. At the beginning of a course acquisition, aimed at effective communication, prevails. The role of learning emerges at later stages in order to make communication not only effective but also formally correct and socio-culturally appropriate. The Council of Europe, the Chinese Confiucius Institute, TESOL, and other international institutions have defined levels of communicative competence in foreign languages. They all share a common trait and that is, that there is a survival level, or threshold level (B1 in the European model) representing the turning point between acquisition and learning. In FL teaching the role of the teacher is peculiar: he or she 
is the only native speaker available to students. The teacher is the living language model, and it is he or she who chooses other authentic language models among the many possibilities offered by the internet, DVD's, songs, films, ITC's and so on. The whole input is in the teacher's hands, as is the choice of activities and, in most cases, assessment.

The use of second and foreign as synonyms creates a lot of confusion as they represent two quite different teaching contexts (Achard, Niemeier 2004). A contribution of this framework may be the proposal of a common terminology in this field, so that this confusion is reduced;

- LF, lingua franca: A lingua franca is used to facilitate international communication and uses a simplified form, but not an oversimplified form as pidgins do. Two thousand years ago the LF was Latin, in the 19th century it was French, today it is English. The growth of English as a Lingua Franca (ELF) is rapidly changing the way it is taught, even though the label 'English as a Foreign Language' (EFL) is still commonly used (two survey articles on research concerning lingua franca teaching are Seidhofer 2004 and Jenkins, Cogo, Dewey 2011; Polyudova 2014 discusses the teaching of English as a lingua franca).

In the ELF context the aim of the student is not to resemble a native speaker, but rather to be understood by everybody, to communicate successfully albeit with little formal accuracy, especially with regards to 'useless' markers such as the third person ' $s$ ', the sequence of tenses, or the past tense of certain irregular verbs; for the same reason lexis is reduced as synonyms are held to be just as 'useless';

- Classical language: Koran Arabic, ancient Greek, Latin and Sanskrit are classical languages. Their prestige comes from ancient texts and traditions, even though they are the mother tongues of no native speaker. They are often referred to as 'dead languages', but the texts written in these languages are still fully alive and still talk to modern men and women. The cultural dimension, whether explicit in the text, or implicit in the lexis, is fundamental in classical language study, and it has no similar role in other language education contexts. If we continue to study Latin and Greek it is to have direct access to a cultural heritage and to the culture from which we originate as Euro-Americans and not because of a special interest in the aorist or the deponent verb forms (as concerns the integration between classical languages and modern languages in language education, see Balboni 2012).

All these contexts, however different, contribute to the same project, that is, to help activate a person's faculty of language. 


\section{Hypothesis 3. Language Education as the Core of General Education, whatever the Cultural Setting}

Human bodies are meant to be instruments of communication involving voice, sight, hearing, body movements and postures. The human mind, moreover, has created many artificial codes.

The semiotic faculty of human beings (see Hypothesis 2) allows them to create and interpret signs, that is, semiotic structures that unite a meaning, signifié in Ferdinand De Saussure's words, and a physical significant such as a sound, a movement, a noise, and so on.

As far as language education is concerned, the core of the model is the role of language as the metalanguage of all other codes and of language itself: language can describe and discuss all codes, while the opposite is impossible. The educational consequence of this model, provided it is true, is extremely important. Since educational communication uses language as its main tool, alone or together with other codes, language education has a key role in the whole educational process, that is, in making self-actualization possible (Hypothesis 4). In other words, language education is the core of general education, whether it is carried out in the mother tongue, in a language of instruction or in a foreign language as in CLIL methodology.

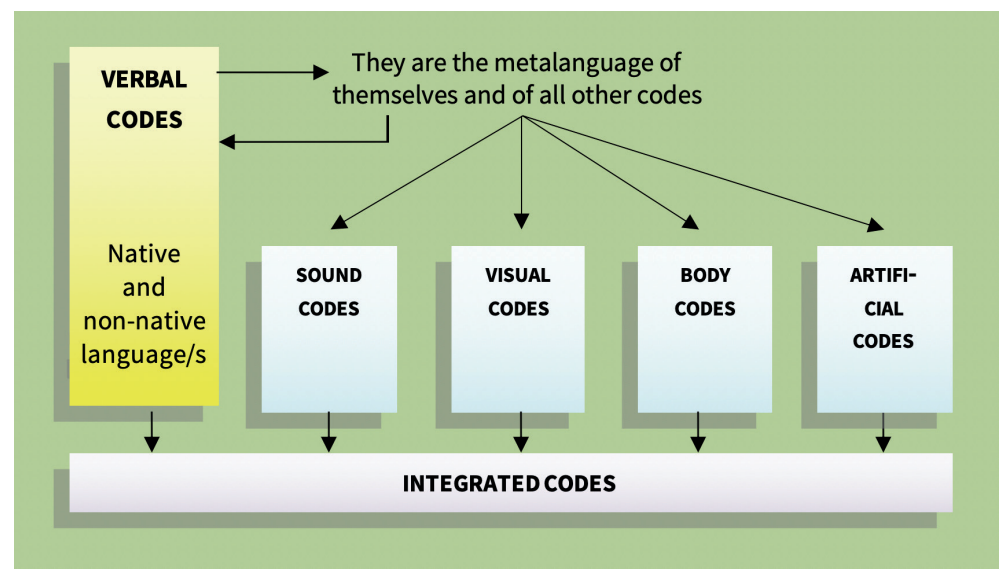

Figure 2 The diagram of semiotic competence

An ethical corollary derives from the model: if it is true that language is the metalanguage of all school subjects, then curricula, syllabi, school organization and language teaching must ensure that all young people are provided not only with mastery of everyday basic interpersonal communication skills (BICS), but also with the highest possible cognitive and academic language proficiency (CALP) in the var- 
ious LSPs, the languages for the specific purposes of communication in mathematics, art, grammar, philosophy, history and so on (studies in educational semiotics can be found in Pesce 2009; Whitson 2009 and Danesi 2012, who focusses on semiotics in language education).

LSPs are fundamental not only as far as learning is concerned, but they also allow for socialization (another objective of language education, Hypothesis 4). In fact, professional and scientific communities are also discourse communities where the LSP communicates that the user belongs to that particular community (new perspectives for LSP teaching under this perspective can be found in Engberg et al. 2007; Gautier 2014; Garzone, Heaney, Riboni 2016).

\section{Hypothesis 4. Language (Education) as a Non-Culture-Bound Condition for Self-Actualization}

Figure 3 draws a map of human relations: human beings interact

a. with themselves in their language of thought (ME, in the diagram),

b. with people they know (relations, work colleagues, friends) or they just meet occasionally (seminars, negotiations, dinners, etc.): people they talk to (YOU, in the diagram), and

c. with the rest of the world, people and facts they talk about (WORLD, in the diagram); it may be today's world or the world of the past, it may be the actual world or the fictional world of literature, and so on.

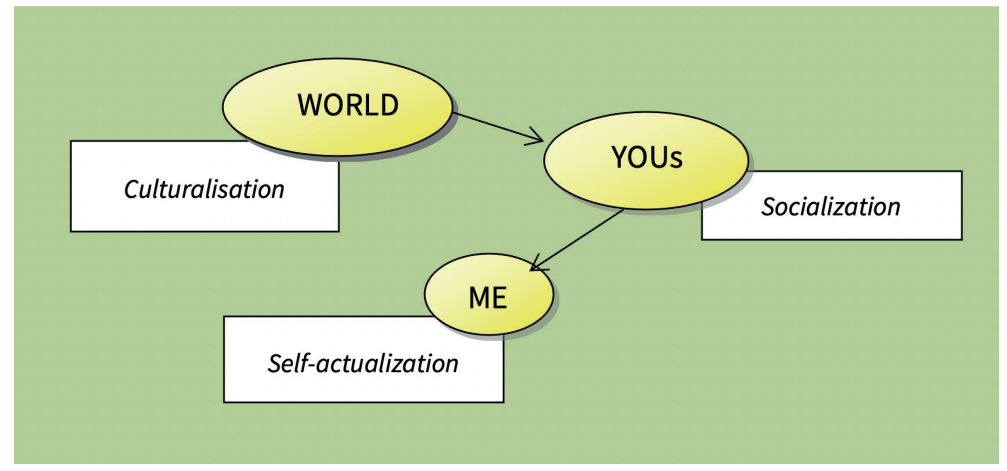

Figure 3 The diagram of human relations

If this model is correct, then it is possible to use it as a foundation in order to build a theory about the objectives of language education, regardless of where it is carried out and independent from culture and tradition. The three goals are: 
a. culturalization. This is the ability of a person to tune in to the culture he or she lives in (inculturation), or has contacts with (acculturation in a second, foreign, ethnic, classical culture). Language is the instrument used in all societies to convey cultural values and behaviours to children and to newcomers: this means that language proficiency is fundamental to culturalization;

b. socialization becomes possible after a child or a newcomer accepts the basic cultural values and models of society (culturalization). The social needs of people, both from an emotional and practical point of view, are met through language, the mastery of which is necessary in order to socialize;

c. self-actualization: this is the fulfilment of one's life project. Only a person who is accepted in the culture he or she lives in (culturalization), and who can socialize with other members of society (socialization) may have the opportunity to aim at self-actualization.

Item 'c' above, self-actualization, is the philosophical core of the whole framework: language is necessary, a conditio sine qua non, for people to pursue self-actualization, the fulfilment of their life project. Language is necessary even to think of a life project.

As a consequence, when translating education into teaching, i.e., when planning syllabi, organizing courses and implementing teaching, the ethical imperative of fulfilling these three objectives is the lodestar.

\section{Hypothesis 5. A Non-Culture-Bound Model of Communicative Competence and Performance}

The meaning of 'knowing a language' is intuitive, yet intuition may produce the sun-circling-the-earth effect. Intuition is, above all, based on tradition and, that is, on a cultural idea of 'knowing' and, as a consequence, a model has to be provided as it is the core of a theory of language education.

Communicative competence (we shall use the traditional expression, although it should be Communicative Competence and Performance) is made up of two components, competence within the mind and performance within the world. Both are governed by the linguistic, extra-linguistic, socio-cultural, and pragmatic conventions accepted by a people, the so-called 'rules'. Acquiring and, subsequently, learning these mental 'rules' make up the first set of objectives which concern language teaching, independently from the language being taught and from the school system in which it is taught.

Another set of objectives is necessary for performance to take place; the development of those cognitive abilities and linguistic 
skills necessary to comprehend, produce, interact, translate, summarize, etc. (the bidirectional arrow uniting the mind and world boxes in figure 4).

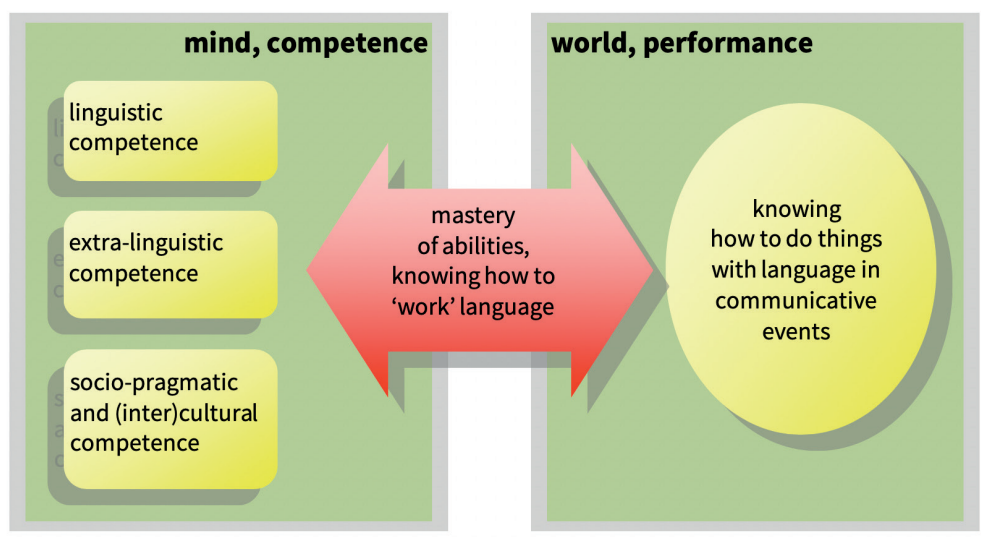

Figure 4 A model of communicative competence and performance

If the model of communicative competence and performance above is right, then:

a. all codes and not just the verbal code are objectives of language teaching: in fact, gestures, expressions, body postures and objects are used in communication together with or even instead of language itself;

b. sociolinguistic, pragmalinguistic and (inter)cultural 'grammars' are objectives of language teaching;

c. the development of language abilities, that is, the cognitive processes underlying language skills, is an objective of language education (this dichotomy can be found in Widdowson 1998).

This hypothesis is central to a theory of language education and teaching. It describes the object, communicative competence, by means of a logical model while in most, if not all, school systems 'knowing a language' is defined according to local traditions. As a consequence, the results of language teaching differ from country to country, and study, work and international mobility are made more difficult. $^{2}$

2 The notion of communicative competence is Hymes', although it was also used by other authors in the early 1970s. There have been many different models of communicative competence. Canale and Swain (1980) set the standard definition for their decade, Bachman (1990) for the following one. Bagarić, and Mihaljević Djigunović (2007) survey the early evolution of the notion of communicative competence. 


\section{Hypothesis 6. A Non-Culture-Bound Model of Intercultural Communicative Competence and Performance}

When participants in a communicative event belong to different cultures, potential intercultural critical points must be detected so that communication does not lead to conflict. Making students aware of the existence of intercultural critical points and of the way to detect and face them is an objective of non-native language teaching.

This hypothesis applies differently to second, foreign, ethnic and classical language teaching as well as to the teaching of lingua francas, such as English today:

a. in second, foreign, ethnic and classical language teaching the student belongs to language/culture ' $\mathrm{A}$ ' and studies language/ culture ' $B$ '. Here the critical points of intercultural communication between ' $\mathrm{A}$ ' and ' $\mathrm{B}$ ' can be known and the appropriate behaviour can be taught;

b. in lingua franca teaching, student ' $A$ ' learns the lingua franca in order to use it in communicative events with people from all languages/cultures. In this case, critical points cannot be detected a priori and cannot be taught. The objective in this case is to raise students' awareness of some of the general categories, and to teach them to observe intercultural communication in a lifelong learning perspective so that they may classify their personal experience according to the model. In other words, they must learn how to write their own textbook of intercultural competence, on the basis of the existing intercultural maps.

The intra-linguistic communicative competence and performance diagram in Hypothesis 5 must be integrated with a map of potential critical points. Figure 4 offers an example of an intercultural communication observation grid; the full model can be found in Balboni, Caon 2014 


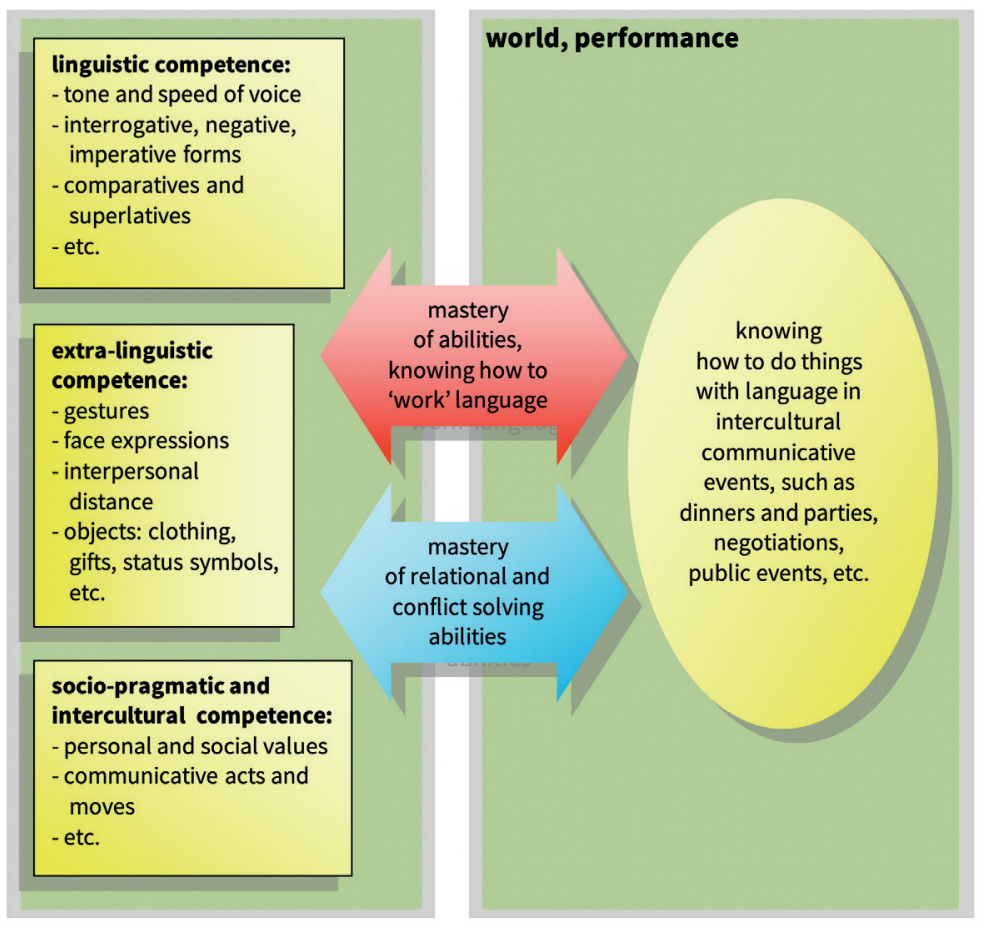

Figure 5 A model of intercultural communicative competence and performance

Both figure 4 and figure 5 describe models, the assumption being that they describe communicative competence and performance in any place or point in time. They are not culture-bound and thus universal as all models claim to be.

As a consequence, all traditional syllabi which do not include, for instance, extralinguistic competence or communicative moves among their objectives should start a revision process, however slow and delicate it may be, as educational systems and education decision-makers are usually rather defensive when radical transformations are proposed.

\section{Hypothesis 7. An Epistemological Model for Educational Linguistics}

A theory must make its epistemological foundations clear and explicit so that users may evaluate both the premises and how it has been built. Two issues need to be solved: 
a. where does the knowledge used to build the framework come from? Is there a universal thesaurus of knowledge the edulinguist may use? Are there any non-culture-bound elements in the repertoire of available knowledge? (Hypothesis 7); and,

b. how is such knowledge organized? Are all the elements on the same plane or is there a hierarchy? (Hypothesis 8).

Today, it is commonly accepted that Edulinguistics draws from four research areas as can be seen from the tables of contents of manuals and readers concerning language education and teaching (see footnote 1). These are: (a) linguistics and all its branches; (b) socio-cultural sciences which study the object of language/culture teaching (Hypotheses 5 and 6); (c) neuro- and psychological sciences which study the subject of language education, that is, those whose faculty of language must be helped to emerge (Hypothesis 2) and who aim at selfactualization (Hypothesis 4); and, (d) pedagogy and teaching methodology which provide the educational knowledge necessary to teach.

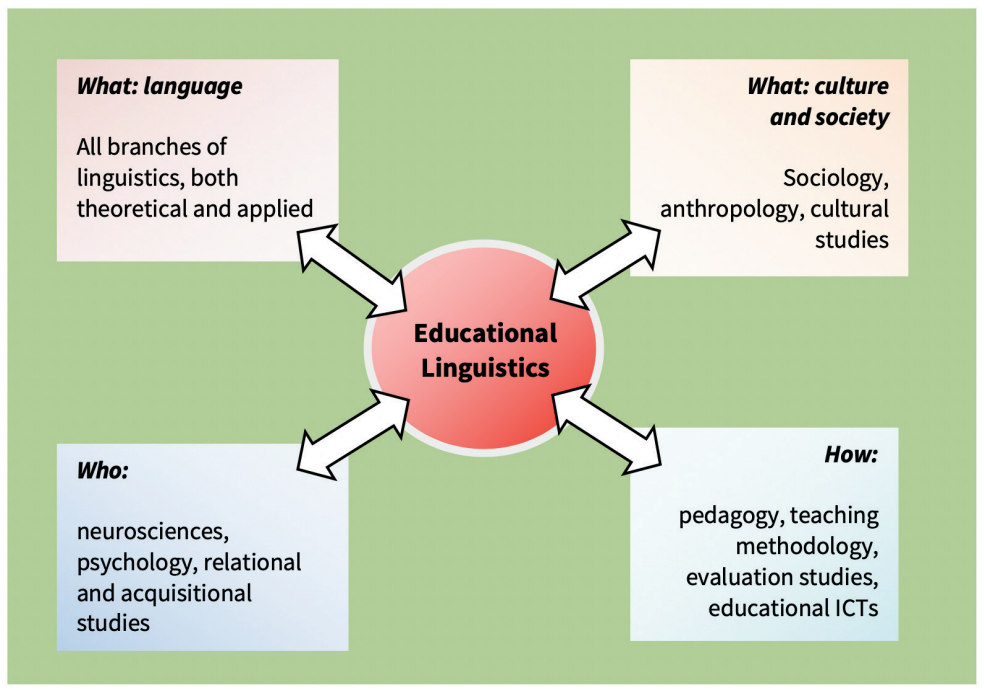

Figure 6 The epistemological diagram of educational linguistics

The traditional view is that information flows from the four research areas towards educational linguistics on the basis of the application principle: the linguist decides what to apply to language education and teaching, and how. This approach leads to applied linguistics, applied psycholinguistics, and language pedagogy. In application, the decision-maker falls outside the realm of educational linguistics. The opposite principle, implication, claims that the decision-makers, who 
analyse their needs and objectives and check whether the knowledge they need is available in the outer research areas, are the educational linguists.

Implication is fundamental for a non-culture-bound framework. Once edulinguists agree on the principles, they may cancel cultural traits from the information they draw from outer sciences. These are especially relevant for the educational box in the diagram.

In picture 6 the bidirectional arrows connecting the four research areas to educational linguistics state that knowledge flows from the outer areas to the inner sphere, and feedback or new research questions flow the opposite way.

\section{Hypothesis 8. A Non-Culture-Bound Model of Research and Action in Educational Linguistics}

Once knowledge has been gathered (Hypothesis 7), it must be organized according to a hierarchical principle indicating which elements are of primary importance, and which ones are secondary.

Traditional models have three levels: approach (what is language education? what does it aim at?); method (how is language education organized, in order to be implemented?); and technique (what is actually done in a class) (Anthony 1963; Puren 2010). In such a tripartite structure, the border between method and technique is rather blurred and the relation between educational linguistics and pedagogical and methodological studies is even hazier. Our theory chooses to consider only two hierarchical levels, approach and method, leaving teaching techniques out of its scope both in order to avoid ambiguity and because teaching techniques depend heavily on culture and tradition.

A theory claiming to be a universal reference point must leave any practical implementation to those who actually teach the language, provided they act consistently with the organizational lines of the method and the principles of the approach.

The graph in figure 7 translates the above into a visual diagram. 


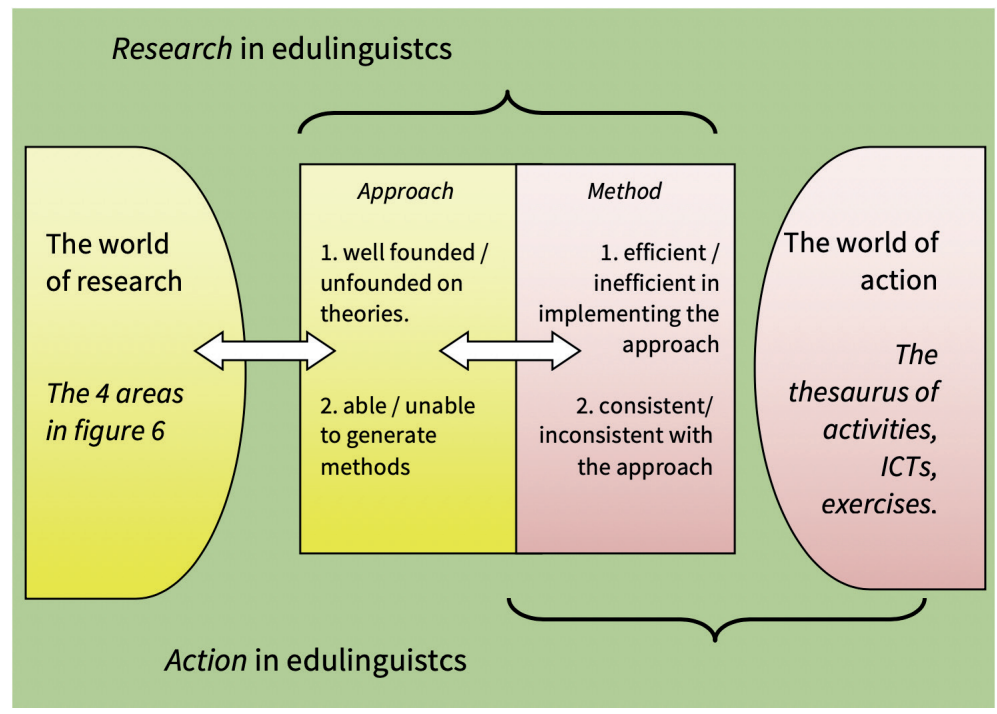

Figure 7 Research and action in Educational Linguistics

The model reads as follows:

a. there is a world of research outside the field of edulinguistic research;

b. approach: this is a theory of language education and not of language teaching. It provides definitions of the following: education, its general aims and specific objectives; language; the learner; and, the teacher;

c. method links language education to language teaching. It provides guidelines for syllabus design, for the nature and the formats of teaching materials, for the role, nature and procedures of classroom teaching and of testing, evaluation and certification as well as for ICT use;

d. the brace over the two boxes includes approach and method as the field of language education and language teaching research (that is, the field of this theory); this area is potentially free of culture-bound elements;

e. the brace at the bottom of the diagram includes the field of language teaching action (not research). Suggestions and guidelines deriving from methodology as do those teaching techniques, which come from the thesaurus of teaching techniques, feed into this field and fall outside the scope of this theory. This area is heavily culture-bound. 


\section{Bibliography}

Achard, M.; Niemeier, S. (eds) (2004). Cognitive Linguistics, Second Language Acquisition, and Foreign Language Teaching. Berlin; New York: Mouton De Gruyter.

Allwein, G.; Barwise, J. (eds) (1996). Logical Reasoning with Diagrams. New York; Oxford: Oxford University Press.

Anthony, E.M. (1963). "Approach, Method and Technique". English Language Teaching, 17(2), 63-7. https://doi.org/10.1093/elt/xvii.2.63.

Bachman, L.F. (1990). Fundamental Considerations in Language Testing. Oxford: Oxford University Press.

Bagarić, V.; Mihaljević Djigunović, J. (2007). "Defining Communicative Competence". Metodika, 8(1), 94-103.

Balboni, P.E. (2011a). Verità, conoscenza, etica nell'educazione linguistica (Truth, Knowledge, Ethics in Language Education). Perugia: Guerra.

Balboni, P.E. (2011b). Language Teaching Research Based on the Theory of Models. Perugia: Guerra.

Balboni, P.E. (2012). Fare educazione linguistica: insegnare italiano, lingue straniere, lingue classiche (Implementing Language Education. Teaching Italian L1/L2, Foreign Languages, Classical Languages). Torino: UTET Università.

Balboni, P.E. (2018). A Theoretical Framework for Language Education and Teaching. Newcastle Upon Tyne: Cambridge Scholars.

Balboni, P.E.; Caon, F. (2014). "A Performance-Oriented Model of Intercultural Communicative Competence”. Journal of Intercultural Communication, 35. http://www.immi.se/intercultural/nr35/balboni.html.

Brinton, D.M.; Kagan, O.; Bauckus, S. (eds) (2008). Heritage Language Education. A New Field Emerging. New York: Routledge.

Clapham, C. et al. (eds) (1997). Encyclopedia of Language and Education. Dortrecht: Kluwer.

Canale, M.; Swain, M. (1980). "Theoretical Bases of Communicative Approaches to Second Language Teaching and Testing”. Applied Linguistics, 1(1), 1-47. https://doi.org/10.1093/applin/i.1.1.

Chapelle, C.A. (ed.) (2012). The Encyclopedia of Applied Linguistics. Hoboken (NJ): Wiley Online Publications. https://onlinelibrary.wiley.com/ doi/book/10.1002/9781405198431.

Chomsky, N.; Hauser, M.D.; Fitch, T.W. (2002). "The Faculty of Language: What Is It, Who Has It, and How Did It Evolve?". Science, 298(5598), 1569-79. https://doi.org/10.1126/science.298.5598.1569.

Danesi, M. (2012). Semiotics in Language Education. Boston: Mouton De Gruyter. Davies, A. (2003). Native Speaker. Myth and Reality. Clevedon: Multilingual Matters. Engberg, J. et al. (eds) (2007). New Directions in LSP Teaching. Berlin: Peter Lang.

Garzone, G.; Heaney, D.; Riboni, G. (eds) (2016). "Focus on LSP Teaching. Developments and Issues". Annual Monograph Issue of Lingue Culture Mediazioni. Languages Cultures Mediations, 1.

Gautier, L. (2014). "Des langues de spécialité à la communication spécialisée. Un nouveau paradigme de recherche à l'intersection entre sciences du langage, info-com et sciences cognitives?" (From LSP to Specialized Communication. A New Research Paradigm Across Language Sciences, Information-Communication Research and Cognitive Sciences). Etudes interdisciplinaires en sciences humaines, 1, 225-45. 
Holyoak, K.J. (2012). "Analogy and Relational Reasoning". Holyoak, Morrison 2012, 234-59.

Holyoak, K.J.; Morrison, R.G. (eds) (2012). The Oxford Handbook of Thinking and Reasoning. New York: Oxford University Press.

Hornberger, N.H. (ed.) (2008). Encyclopedia of Language and Education. New York: Springer.

Jenkins, J.; Cogo, A.; Dewey, M. (2011). "Review of Developments in Research into English as a Lingua Franca". Language Teaching, 44(3), 281-315. https://doi.org/10.1017/S○261444811000115.

Johnson-Laird, P.N. (2002). "Pierce, Logic Diagrams, and the Elementary Operations of Reasoning". Thinking and Reasoning, 8(1), 69-95. https://doi. org/10.1080/13546780143000099.

Pesce, S. (2009). "From Semiotics of Teaching to Educational Semiotics: The Case of French-Speaking Research in Education". Semetsky 2009, 99-114. https://doi.org/10.1163/9789460912252_008.

Polyudova, E. (ed.) (2014). Acquiring Lingua Franca of the Modern Time. Current Issues and Strategies in ESL Studies. Newcastle Upon Tyne: Cambridge Scholars.

Puren, C. (2010). "Domaines de la didactique des langues-cultures. Entrées libres" (Domains in Educational Linguistics. Free Entrance). http://www. gerflint.fr/Base/AmeriqueduNord1/Domaines.pdf.

Rothmaler, P. (2000). Introduction to Model Theory. Amsterdam: Gordon and Breach.

Seidlhofer, B. (2004). "Research Perspectives on Teaching English as a Lingua Franca". Annual Review of Applied Linguistics, 24, 209-39. https://doi. org/10.1017/s@267190504000145.

Semetsky, I. (ed.) (2009). Semiotics Education Experience. Rotterdam: Sense.

Spolsky, B.; Hult, F.M. (eds) (2007). The Handbook of Educational Linguistics. Boston: Blackwell.

Van Deusen-Scholl, N. (2003). "Toward a Definition of Heritage Language: Sociopolitical and Pedagogical Considerations". Journal of Language, Identity, and Education, 2(3), 211-30. https://doi.org/10.1207/ s15327701jlie0203_4.

Vollmer, H.J. (2006a). Vers un instrument européen commun pour la / les langue(s) de scolarisation (Towards a Common European Instrument for the Language(s) of Instruction). Strasbourg: Conseil de l'Europe.

Vollmer, H.J. (2006b). Langues d'enseignement des disciplines scolaires (Languages of Instruction and School Subjects). Strasbourg: Conseil de l'Europe.

Whitson, J.A. (2009). "Curriculum as Semiotic Formation. From Signifying Discourses, to the Formation of Human Being". Semetsky 2009, 83-98. https://doi.org/10.1163/9789460912252_007.

Widdowson, H.G. (1998). "Skills, Abilities, and Contexts of Reality". Annual Review of Applied Linguistics, 18, 323-33. https://doi.org/10.1017/ s0267190500003615. 



\title{
EL.LE
}

Vol. 9 - Num. 1 - Marzo 2020

\section{Didattica dell'italiano L2 ad apprendenti adulti analfabeti Uno studio di caso all'interno di CPIA, SPRAR e CAS della Provincia di Venezia}

\author{
Annalisa Brichese \\ Università Ca' Foscari Venezia, Italia \\ Camilla Spaliviero \\ Università Ca' Foscari Venezia, Italia \\ Valeria Tonioli \\ Università Ca' Foscari Venezia, Italia
}

\begin{abstract}
This article presents the results of a case study carried out in the Provincial Centres for Adult Education (CPIA), in the Protection System for Refugees and Asylum Seekers (SPRAR) and in the Extraordinary Reception Centres (CAS) in the province of Venice. The article aims at providing a picture of the Italian courses offered to CPIA illiterate students who are beneficiary of CAS and SPRAR. In particular, the article explores the teachers' profiles, the materials, the contents and the methodologies, the assessment methods and the feedback strategies. Quantitative data were collected through questionnaires.
\end{abstract}

Keywords Teaching Italian as L2. Illiterate students. Adult education. Case study. Migrant students.

Sommario 1 Introduzione. - 2 Metodologia della ricerca. - 2.1 Lo studio di caso. - 2.2 I partecipanti. - 2.3 La raccolta delle informazioni. - 3 Analisi dei dati. - 3.1 Esperienza e formazione specifica. - 3.2 Metodologia utilizzata. - 3.3 Contenuti e risorse. - 3.4 I metodi di valutazione/testing. - 3.5 La gestione del feedback nell'oralità e nella scrittura - 4 Discussione e conclusioni - 4.1 II profilo del docente; 4.2 La metodologia didattica. - 4.3 Contenuti e volumi. - 4.4 I metodi di valutazione/testing. - 4.5 La gestione del feedback nell'oralità e nella scrittura.

\begin{tabular}{lll} 
& \multicolumn{2}{l}{ Peer review } \\
& Submitted & $2019-04-18$ \\
Edizioni & Accepted & $2019-09-28$ \\
Ca'Foscari & Published & $2020-07-30$
\end{tabular}

\section{Open access}

(C) 2020 | (9) Creative Commons Attribution 4.0 International Public License

Citation Brichese, A.; Spaliviero, C.; Tonioli, V. (2020). "Didattica dell'italiano L2 ad apprendenti adulti analfabeti. Uno studio di caso all'interno di CPIA, SPRAR e CAS della Provincia di Venezia". EL.LE, 9(1), 25-56. 


\section{Introduzione}

Nel presente contributo intendiamo presentare un'indagine condotta nei Centri Provinciali per l'Istruzione degli Adulti (CPIA), nei servizi erogati dal Sistema di Protezione per Richiedenti Asilo e Rifugiati (SPRAR) e nei Centri di Accoglienza Straordinaria (CAS) all'interno della Provincia di Venezia con l'obiettivo di ricercare:

a. informazioni su esperienza e formazione specifica dei docenti di italiano come seconda lingua (d'ora in poi L2) ad adulti analfabeti nella propria lingua madre (d'ora in poi L1);

b. la metodologia utilizzata dai docenti all'interno dei corsi con studenti analfabeti;

c. i contenuti e le risorse utilizzate dai docenti nei corsi summenzionati;

d. i metodi di valutazione/testing adoperati;

e. i metodi di correzione dell'errore utilizzati.

Nello specifico abbiamo ritenuto necessario indagare i seguenti ambiti:

- la formazione degli operatori che conducono corsi di italiano L2 a studenti adulti analfabeti all'interno di CPIA, SPRAR e CAS. La ragione di tale interesse di ricerca è dovuta al fatto che i profili degli operatori che insegnano lingua all'interno dei succitati contesti sono eterogenei. Non esistono, infatti, linee guida nazionali con le quali si identifichino le competenze specifiche dei facilitatori di italiano L2 che lavorano con questo specifico target di apprendenti adulti;

- la metodologia adottata in classe dai facilitatori poiché sono assenti a livello nazionale linee guida relative ai metodi da impiegare nell'insegnamento dell'italiano L2 per apprendenti analfabeti nella propria L1.

Solo per quanto concerne i rifugiati, ${ }^{1}$ si può far riferimento unicamente al Toolkit del Consiglio d'Europa. Supporto linguistico per rifugiati adulti nel quale vengono forniti alcuni strumenti operativi e proposte didattiche ad operatori del settore. Sono poi presenti studi specifici condotti in Italia sulla didattica ad adulti richiedenti asilo o titolari di protezione internazionale (tra cui Shapiro, Farrelly, Curry 2018; Machetti, Rocca 2017; Zimmari 2014) ma che non possono fungere da linee guida nazionali;

Il contributo è frutto di un lavoro congiunto tra le autrici. Tuttavia, Annalisa Brichese ha curato i paragrafi 2.2, 2.3, 3.1, 3.2, 3.3, 4.1, 4.2, 4.3; Camilla Spaliviero ha curato i paragrafi 3.4, 3.5, 4.4, 4.5; Valeria Tonioli ha curato i paragrafi 1, 2 e 2.1.

1 Intesi in senso ampio e onnicomprensivo, comprendendo sia i richiedenti asilo che i migranti già titolari di protezione internazionale. 
- le risorse impiegate in aula e i contenuti presentati durante il percorso di apprendimento della L2 poiché, come vedremo confermato anche dallo studio di caso, le proposte di manuali didattici specifici per questo target di apprendenti non sono numerose;

- i metodi di valutazione e di correzione dell'errore utilizzati. Rispetto a quest'ultimo punto, nel manuale per la gestione dello SPRAR si afferma l'importanza di strutturare un percorso di valutazione delle competenze e delle conoscenze dei beneficiari. Tuttavia ne viene presentata l'importanza senza fornire dettagli rispetto a come strutturare un percorso valutativo trasversale a livello nazionale:

Il progetto dovrà altresì attrezzarsi per un effettivo monitoraggio circa il percorso di apprendimento dell'italiano dei beneficiari. Dovrà dunque essere elaborato uno strumento per valutare le competenze di italiano acquisite, predisponendo anche un libretto delle competenze linguistiche e del percorso di apprendimento di ciascun beneficiario, dove registrare le frequenze e il livello di apprendimento conseguito. (SPRAR 2015, 47)

La professione di docente di italiano come lingua seconda con target di studenti analfabeti in lingua madre è per sua natura complessa perché è richiesto agli insegnanti di possedere competenze specifiche in molti ambiti. Alfabetizzare in italiano L2 significa occuparsi contemporaneamente di:

- adulti;

- studenti di seconda lingua;

- immigrati e/o rifugiati;

- lettori e scrittori emergenti.

Per ciascuno dei summenzionati ambiti esistono studi specifici a cui i docenti devono far riferimento (si vedano, tra tanti, Knowles et al. 1998; Kern, Schultz 2005; Kurvers 2007; Tarone, Bigelow, Hansen 2009; Vinogradov 2009; Bigelow, Schwarz 2010; Vinogradov, Bigelow 2010; Boon 2014; Borri et al. 2014). Si pensi, ad esempio, alle diverse nozioni di alfabetizzazione, ai significati che assume la definizione di lettori e scrittori emergenti, al ruolo dell'apprendimento strumentale della letto-scrittura rispetto all'acquisizione della seconda lingua. Tutti questi elementi riflettono la natura e l'ambiguità esistente ancora oggi nel campo dell'alfabetizzazione iniziale.

Nonostante la complessità evidenziata, la ricerca sull'identità degli insegnanti alfabetizzatori è carente. Pochi studi hanno esplorato i molteplici ruoli che tali docenti devono ricoprire e il modo in cui essi si formano nell'ottica del Lifelong Learning. Le indagini sull'insegnamento e l'apprendimento che includono il concetto di alfabe- 
tizzazione iniziale si concentrano più spesso sugli studenti che sugli insegnanti (Colliander 2018, 35).

Secondo Vinogradov (2013), l'insegnante di L2, che lavora con studenti analfabeti, dovrebbe possedere:

- la comprensione e conoscenza generale del concetto di alfabetizzazione in questo contesto specifico;

- la conoscenza della lingua oggetto di studio;

- la conoscenza dei processi di acquisizione linguistica;

- l'esperienza specifica di gestione di gruppi composti da immigrati e rifugiati.

Colliander (2018) ricorda che, in questo senso, le competenze dell'insegnante di L2 che si occupa di studenti analfabeti in L1 sembrano assomigliare a quelle di un qualsiasi docente di seconda lingua ma, in realtà, ciò che rende più complesso l'insegnamento a studenti adulti non alfabetizzati è il fatto che i docenti non hanno avuto esperienza di ciò che significa imparare a leggere e scrivere in una lingua non materna da adulti.

L'ancora scarsa ricerca in quest'ambito d'insegnamento specifico porta i docenti alfabetizzatori, in alcuni casi, ad adattare le metodologie legate all'alfabetizzazione iniziale dei bambini applicandole agli studenti adulti (Vinogradov 2013). Gli insegnanti dovrebbero invece considerare le risorse degli studenti - le loro conoscenze, la loro lingua e le loro esperienze - e collegare le attività di insegnamento di alfabetizzazione iniziale a questi aspetti (Vinogradov, Bigelow 2010).

Su questa base, considerata l'eterogeneità del panorama a livello nazionale e l'assenza di uno studio di caso specifico nelle aree della Provincia di Venezia, le domande di ricerca che sono state formulate e impiegate durante l'indagine sono:

a. Qual è il profilo del docente che insegna italiano ad analfabeti?

b. Quali abilità vengono maggiormente sviluppate nella classe di L2 con studenti analfabeti?

c. Quali metodi di letto-scrittura vengono utilizzati all'interno di tali corsi?

d. Come vengono strutturate le attività?

e. Quali argomenti vengono trattati?

f. Quali materiali vengono utilizzati?

g. Come vengono testate le competenze in ingresso, in itinere e in uscita degli studenti analfabeti?

h. Come viene gestita in classe la correzione degli errori compiuti dagli apprendenti analfabeti?

Come requisito preliminare, i docenti intervistati dovevano aver lavorato in corsi di italiano L2 rivolti specificatamente a studenti analfabeti o in corsi multilivello in cui fossero presenti utenti non scolarizzati in L1. 
Dopo aver individuato le domande di ricerca è stata avviata l'indagine così come presentato nel paragrafo successivo.

\section{Metodologia della ricerca}

Per realizzare l'indagine abbiamo impiegato una ricerca descrittiva di tipo qualitativo ed esplorativo in cui, secondo Creswell (2009, 26-7), «il ricercatore tenta di costruire un sapere basato su quanto raccolto dai partecipanti e dall'osservazione compiuta durante la propria indagine». Inoltre, la ricerca descrittiva è necessaria per raccogliere dati e mappare uno specifico contesto di riferimento. Nel caso specifico dell'indagine in oggetto, si intendeva fornire una fotografia dei corsi di italiano rivolti a studenti del CPIA e beneficiari di CAS e SPRAR della Provincia di Venezia. Per svolgere tale ricerca abbiamo condotto uno studio di caso (Flick 2014) in cui raccogliere dati quantitativi tramite la somministrazione di un questionario online in Google drive. La scelta di somministrare un questionario prevalentemente a risposta chiusa è stata determinata dalla necessità di raggiungere quanti più facilitatori e docenti possibile, poter inserire i dati e tabulare i risultati ottenuti all'interno di una tabella.

\subsection{Lo studio di caso}

Secondo Stake (1995), lo studio di caso è una strategia d'indagine nella quale il ricercatore esplora in profondità un programma, un evento, un'attività, un processo di uno o più individui.

Nello specifico della ricerca da noi condotta, si sono indagati la metodologia, le tecniche, i contenuti e i materiali impiegati dai facilitatori e docenti di italiano L1 a beneficiari e studenti analfabeti di CPIA, SPRAR e CAS.

Nel panorama italiano e internazionale è possibile trovare riscontro di alcuni studi relativi alla didattica della lingua seconda con adulti analfabeti in L1 con l'obiettivo di:

- indagare la percezione degli studenti (Elmeroth 2010; Martín Fernández 2013);

- condurre studi empirici descrittivi ed esplorativi nati dall'osservazione all'interno di classi di adulti analfabeti sulla didattica impiegata nell'insegnamento di una L2;

2 Si ricordi, tra tanti, Burt, Kreeft Peyton, Schaetzel 2008; Cardoso-Martins, Rodrigues, Ehri 2003; Choi, Ziegler 2015; Colliander 2018; Cronen et al. 2011; Eme 2011; Fulanete Corrêa, Cardoso-Martins, Rodrigues 2010; Tammelin-Laine, Martin 2014. 
- condurre ricerche specifiche sulla didattica ad adulti richiedenti asilo e rifugiati (Benseman 2012);

- esplorare le strategie di gestione del feedback nell'oralità e nella scrittura; ${ }^{3}$

- indagare le strategie di lettura in giovani analfabeti nella propria L1 (Benítez, Borzone 2012);

- indagare e analizzare gli errori commessi da 15 studenti adulti analfabeti che stanno imparando a leggere e scrivere (Calcagno et al. 2016);

- presentare sillabo e descrittori per la didattica della L2 ad analfabeti adulti (Borri, Minuz 2016).

\subsection{I partecipanti}

I partecipanti coinvolti nello studio sono stati complessivamente 32 e sono tutti facilitatori di italiano L2 che lavorano all'interno di CAS e SPRAR e docenti di italiano L2 impiegati nei CPIA della Provincia di Venezia.

Dal sito della prefettura ${ }^{4}$ è stato raccolto l'elenco degli enti gestori affidatari di progetti CAS e SPRAR che risultano essere in tutto 28, suddivisi nei diversi comuni della provincia di Venezia.

Tutte le sedi CAS e SPRAR sono state contattate telefonicamente al fine di:

- definire se i centri di accoglienza organizzano direttamente i corsi all'interno delle proprie strutture oppure si appoggino al CPIA nei mesi da ottobre a maggio e ai corsi organizzati da altri enti (comuni, associazioni di volontariato del territorio, ecc.) durante il periodo estivo;

- richiedere gli indirizzi mail del referente dei corsi di italiano e/o dei facilitatori di italiano L2 che conducono le lezioni.

Delle 28 sedi contattate, 3 sono risultate irreperibili sia telefonicamente sia via mail e 3 non organizzano direttamente i corsi all'interno delle proprie strutture ma si appoggiano ad altri dispositivi offerti dal territorio.

Dal sito dei Centri Provinciali per l'istruzione agli Adulti ${ }^{5}$ sono state reperite le sedi dei CPIA territoriali, complessivamente 7, distribuiti tra Venezia (che comprende anche la sede carceraria), Mestre, Marghera, Dolo, Spinea, Mirano e San Donà di Piave.

3 Ad esempio, si veda in ordine cronologico, Comings, Soricone 2005; Faux 2006; Kreeft Peyton, Schaetzel, Burt 2007; Mathews-Aydinli 2008; Tarone, Bigelow, Hansen 2009; Vinogradov 2009.

4 http://www.prefettura.it/venezia/contenuti/Enti_gestori-638571.htm.

5 https://cpiavenezia.edu.it. 
Tutti i 14 docenti d'italiano L2 che lavorano con studenti analfabeti sono stati contattati via mail.

\subsection{La raccolta delle informazioni}

La raccolta delle informazioni è avvenuta tramite un questionario in drive che è stato inviato tramite mail ai contatti reperiti attraverso le modalità presentate in 2.2 .

Il questionario è stato aperto il 9 aprile e chiuso il 30 giugno per consentire a tutti i facilitatori e docenti coinvolti di compilarlo compatibilmente con gli impegni lavorativi di ciascuno. I questionari sono anonimi anche se vi è richiesta specifica di indicare il luogo in cui i docenti operano.

Per ognuna delle domande e coerentemente con gli obiettivi della ricerca (cf. § 1), sono stati strutturati specifici item all'interno di un questionario.

\section{$3 \quad$ Analisi dei dati}

In totale sono state raccolte 32 risposte dei partecipanti che hanno risposto al questionario anonimo online. Di seguito si riportano i dati ricavati dalle domande relative ai seguenti obiettivi della ricerca:

- raccogliere informazioni su esperienza e formazione specifica (punto 1);

- individuare la metodologia utilizzata dai docenti all'interno dei corsi (punto 2);

- individuare contenuti e risorse utilizzate dai docenti nei corsi. (punto 3);

- individuare i metodi di valutazione/testing adoperati (punto 4);

Individuare le strategie di gestione del feedback nell'oralità e nella scrittura (punto 5).

\subsection{Esperienza e formazione specifica}

La prima sezione del questionario è dedicata alla formazione dei docenti e facilitatori di L2 in didattica della lingua a stranieri e/o a stranieri analfabeti. Di seguito si analizzano i dati raccolti. 
Dove lavora?

Con questa domanda ci si propone di avere una panoramica sulla distribuzione dei docenti che hanno aderito allo studio nelle diverse Istituzioni pubbliche e private che offrono corsi di italiano a studenti analfabeti, come si può osservare dalla figura 1 ,

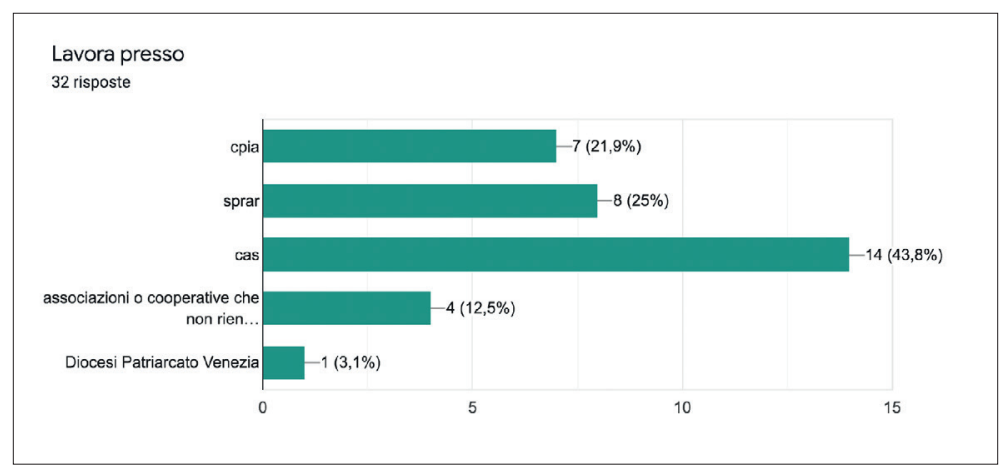

Figura 1 Risposte del questionario: luogo di lavoro dei facilitatori e/o docenti di italiano

La maggior parte degli informatori (14; 43,8\%), lavora presso un CAS mentre il 25\% in uno SPRAR. Nei CPIA si trova il 21,9\% di coloro che hanno risposto al questionario mentre il 15,6\% degli informatori è impiegato in associazioni, cooperative o Diocesi del Patriarcato di Venezia che non fanno parte delle 3 categorie sopra elencate. Sommando le risposte si arriva ad un totale di 34 risposte anziché 32: ciò significa che o 2 informatori lavorano contemporaneamente in due centri diversi o che un intervistato si divide fra 3 centri differenti.

\section{Da quanto tempo insegna italiano L2 a studenti analfabeti?}

Con questa domanda si vuole sondare l'esperienza lavorativa specifica nell'ambito oggetto d'indagine. Come emerge dalla figura 2, la maggioranza degli informatori (75\%) insegna da meno di 3 anni mentre il 12,5\% insegna dai 7 ai 9 anni. Il 6,3\%, invece, insegna da 4 a 6 anni e solo il restante $6,2 \%$ da oltre 10 anni [fig. 2]. 
Da quanto tempo insegna italiano $\mathrm{L} 2$ a studenti analfabeti? 32 risposte

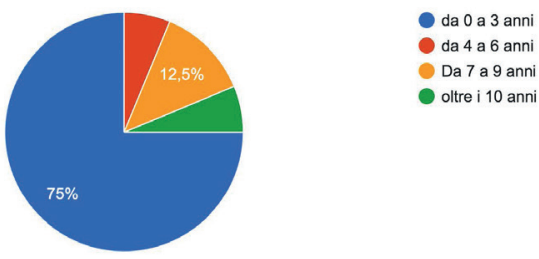

Figura 2 Risposte del questionario: esperienza lavorativa specifica in contesto d'insegnamento dell'italiano L2 a studenti analfabeti

Ha una formazione specifica in italiano L2?

Attraverso questa domanda si vuole sondare se i docenti abbiano una formazione specifica sulla didattica a studenti stranieri in contesto di apprendimento di lingua seconda. Dai dati visibili in figura 3, emerge che l' $81,3 \%$ ha una formazione specifica sull'insegnamento dell'italiano L2 mentre il 18,8\% non ha alcuna formazione in questo settore [fig. 3].

Ha una formazione specifica in italiano L2?

32 risposte

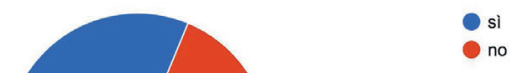

Figura 3 Risposte del questionario: formazione specifica dei docenti nell'insegnamento dell'italiano L2

Ha una formazione specifica nella didattica dell'italiano a studenti analfabeti?

Tramite questa domanda, infine, si vuole verificare non solo che i docenti abbiano una formazione specifica sulla L2 ma che abbiano approfondito gli studi sulla didattica ad adulti analfabeti in lingua madre. Dalla figura 4 emerge che il 59,4\% degli informatori non ha una formazione in didattica dell'italiano come L2 rivolta a studenti analfabeti mentre il 40,6\% ha una formazione specifica in questo settore [fig. 4]. 


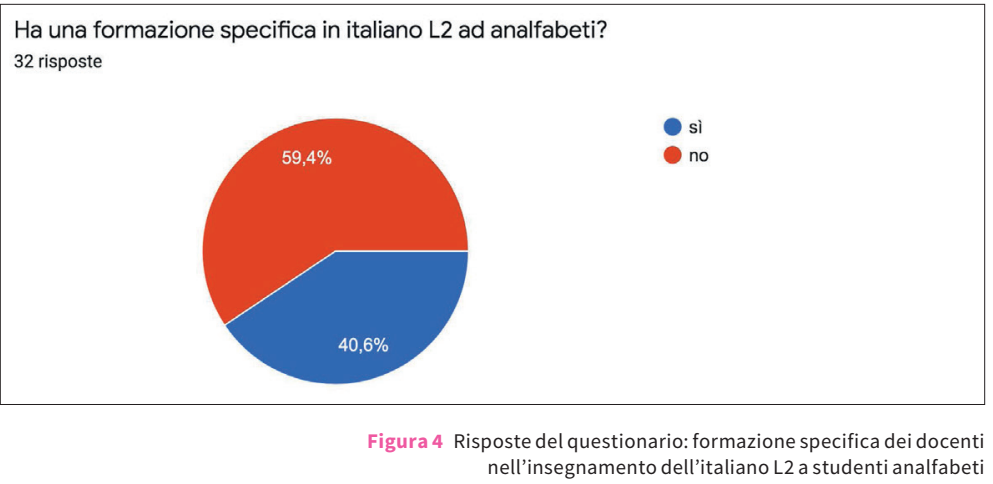

\subsection{Metodologia utilizzata}

La seconda parte del questionario era invece rivolta ad indagare la metodologia impiegata in classe da parte dei docenti e/o facilitatori di L2 con studenti analfabeti. Di seguito si riportano tutte le domande relative a questa sezione con le relative analisi dei dati.

\section{Quale modalità di lavoro utilizza maggiormente in classe con studenti analfabeti?}

Con questa domanda si vuole rilevare con quale modalità prevalente il docente organizzi la lezione. In particolare se prediliga un lavoro in plenum, a gruppi, a coppie o individuale. Come si può osservare nella figura 5, il 43,8\% degli informatori organizza la lezione a partire da una spiegazione in plenaria per poi passare al lavoro individuale; il 28,1\% lavora prima in plenaria e poi a gruppi; il 21,9\% lavora prima in plenaria e poi a coppie. Del 6,2\% degli informatori che risponde «altro», il 3,1\% precisa che la scelta dipende dalla composizione del gruppo classe, che spesso è misto (per genere e livello linguistico) mentre il restante 3,1\% segue individualmente gli studenti per rispondere in modo più adeguato ai diversi ritmi di lavoro nell'esecuzione delle attività e tipologie di difficoltà e alla criticità legata alla presenza discontinua degli studenti a lezione [fig. 5]. 


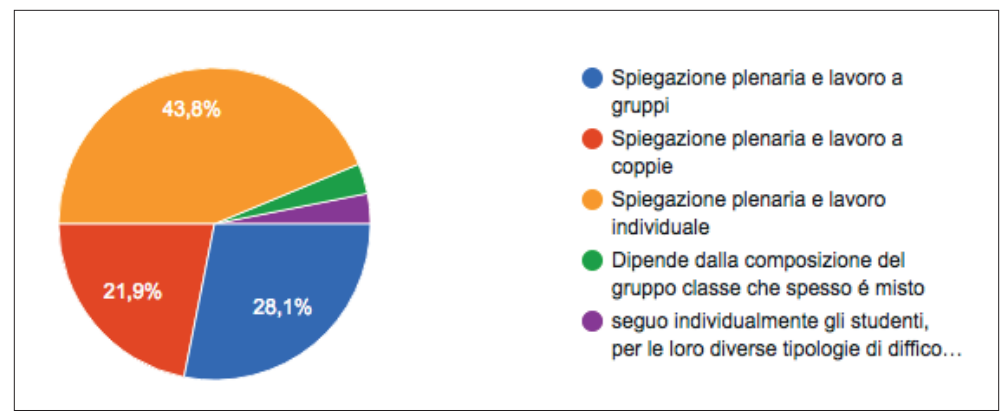

Figura 5 Risposte del questionario: modalità di lavoro prevalente nelle classi con studenti analfabeti

Su quale abilità ritiene che gli analfabeti debbano lavorare di più all'inizio del percorso (di acquisizione linguistica)?

Con questa domanda si vuole indagare se ci sia un'abilità che, secondo il campione degli informatori, necessiti di maggior attenzione e tempo ad inizio del percorso di acquisizione linguistica. In particolare se lo studente analfabeta neoarrivato abbia bisogno di sviluppare primariamente le abilità orali (interazione, produzione e comprensione orali) o le abilità scritte (interazione, produzione e comprensione scritte).

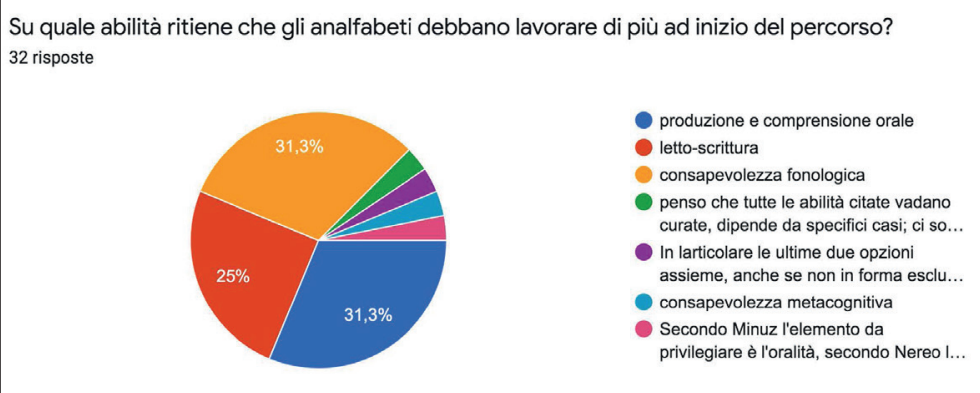

Figura 6 Risposte del questionario: abilità prevalente da sviluppare in aula con studenti analfabeti ad inizio del percorso di L2

Dalla figura 6 possiamo dedurre che il 62,6\% degli informatori si divide tra un $31,3 \%$ che, inizialmente, privilegia un lavoro che sviluppi le abilità di comprensione e produzione orale e un altro 31,3\% un lavoro che sviluppi invece la consapevolezza fonologica. Il 25\% degli informatori ritiene sia fondamentale concentrarsi sulle competenze tecniche di letto-scrittura fin dall'inizio del percorso di apprendimento. Il restante $8,4 \%$ ha risposto «altro» fornendo motivazioni diverse. Alcuni specificano infatti che: 
- sia importante lavorare su abilità integrate;

- sia fondamentale privilegiare la letto-scrittura e la consapevolezza fonologica anche se non in forma esclusiva;

- sia necessario dare spazio alla consapevolezza metacognitiva. Un solo intervistato non prende una posizione definita affermando che anche la letteratura di settore non è concorde su questo tema.

\section{Quale abilità ritiene debba essere maggiormente potenziata in un percorso per analfabeti?}

Con questa domanda si vuole sondare se il docente ritenga necessario sviluppare o potenziare maggiormente le abilità orali, scritte 0 la consapevolezza fonologica. Come si può osservare nella figura 7 , il 68,8\% degli informatori ritiene che il percorso di alfabetizzazione per studenti analfabeti debba concentrarsi principalmente sul potenziamento della letto-scrittura; il 15,6\% sulla consapevolezza fonologica; il 12,5\% sulla produzione e comprensione orale. Il restante 3,1\% risponde «altro» specificando che lo sviluppo di tutte le abilità deve essere integrato in modo graduale [fig. 7].

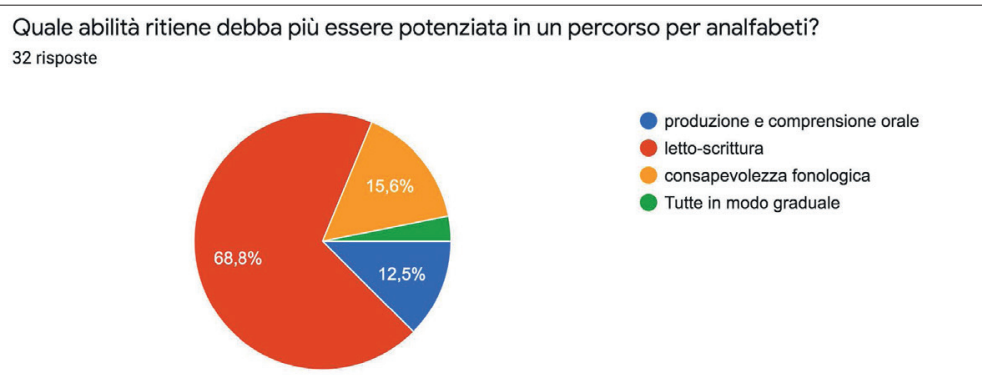

Figura 7 Risposte del questionario: abilità da potenziare in un percorso di L2 per studenti analfabet

Come attiva (cioè introduce, presenta) la parte dedicata alla lettura e alla scrittura? (sono possibili più risposte)

Tramite questa domanda, che prevede la possibilità di indicare più risposte, si indaga come il docente introduca le attività di acquisizione della lettura e della scrittura. In particolare se si utilizzino materiali autentici oppure parole note, sillabe o lettere scritte alla lavagna o presenti nel libro di testo adottato. La maggior parte degli informatori (22; 68,8\%) introduce le attività di letto-scrittura attraverso parole note scritte alla lavagna o contenute nel libro di testo; il 62,5\% utilizza materiale autentico di vario tipo; il $43,8 \%$ usa sillabe scritte alla lavagna o presenti nel libro di testo; il 21,9\% scrive singole lette- 
re alla lavagna; il 15, 5\% risponde «altro» specificando che utilizza:

- materiale autentico per la ricerca e il riconoscimento di sillabe;

- immagini proiettate col PC;

- materiale autentico didattizzato;

- acrostici; lo 'scarabeo' sillabico; cartelloni;

- materiale costruito appositamente in base ai bisogni-interessi-etnia degli studenti.

I dati sono visibili in figura 8.

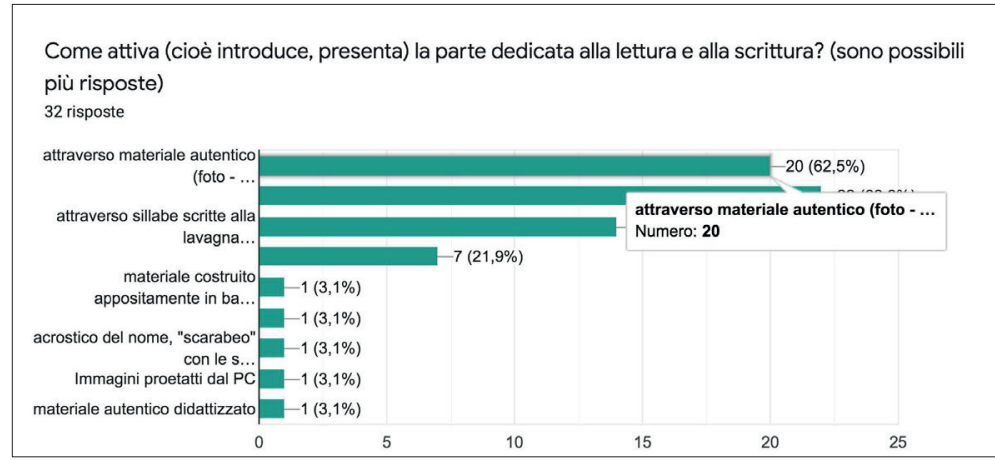

Figura 8 Risposte del questionario: introduzione in classe alla lettura e scrittura per studenti analfabeti

Come lavora sulla decodifica (cioè riconoscere e saper leggere)? Metta una crocetta

Con questa domanda si vuole sondare attraverso quale metodo (analitico-sintetico, globale, integrato) il docente lavori sull'abilità di lettura.

Come lavora sulla decodifica (cioè riconoscere e saper leggere)?

32 risposte

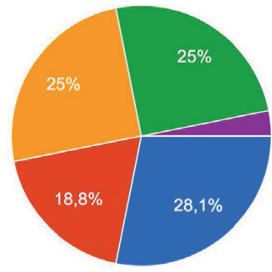

dall'intera parola alla sillaba (non $\mathrm{mi}$ concentro subito sulla lettera singola)

dall'intera parola alla sillaba e alla letter

- dalla sillaba all'intera parola (non $\mathrm{mi}$ concentro sulla lettera singola ma parto dalla sillaba)

dalla lettera alla sillaba allintera parola

Dipende dal livelli di alfabetizzazione pregressa

Figura 9 Risposte del questionario: modalità di lavoro sulla decodifica con studenti analfabeti

Come emerge dalla figura 9, il 28,1\% degli informatori lavora sulla decodifica a partire dalla parola, per poi passare all'analisi di una o più 
sillabe in essa contenuta/e; il 25\%, presenta prima la sillaba per poi arrivare alla lettura della parola intera; il 18,8\% inizia dalla lettura della parola, per poi concentrarsi prima sulle sillabe e poi sulle lettere che la compongono; il 25\% introduce la lettura della lettera, passando poi per la sillaba e arrivando alla fine all'intera parola; il 3,1\% risponde «altro», affermando che dipende dai livelli di alfabetizzazione pregressa [fig. 9].

\section{Come lavora sulla scrittura? Metta una crocetta}

Tramite questa domanda si indaga attraverso quale metodo (analitico-sintetico, globale, integrato) il docente lavori sull'abilità di scrittura. Si presentano i dati emersi in figura 10.

Il $62,6 \%$ degli informatori si divide tra un $31,3 \%$ che lavora sulla scrittura a partire dalla sillaba per poi arrivare a comporre la parola intera e un altro $31,3 \%$ che si concentra prima sulla lettera, poi sulla sillaba e solo infine sulla parola; il 18,8\% lavora sulla scrittura a partire dall'intera parola, passando per la sillaba fino ad arrivare alla lettera; il 15,6\% passa dall'intera parola alla sillaba; il 3,1\% risponde «altro» specificando che lavora in modo integrato ovvero dalla parola alla sillaba e dalla sillaba alla parola, a seconda delle diverse fasi della lezione in cui si trova [fig. 10].

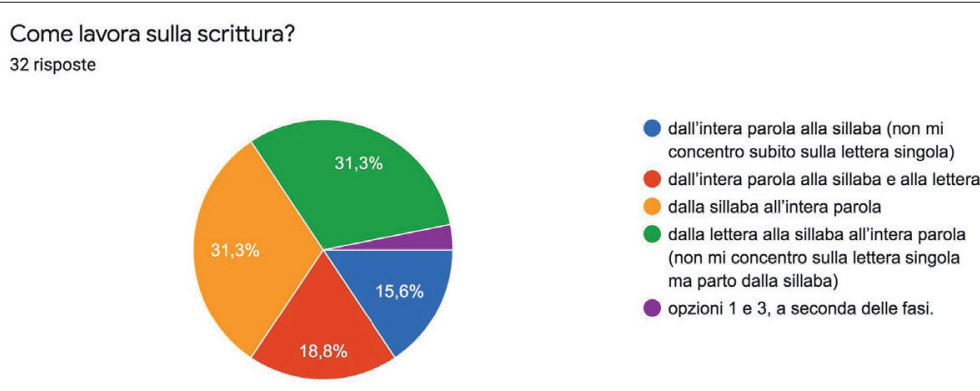

Figura 10 Risposte del questionario: modalità di lavoro sulla scrittura con studenti analfabeti

\subsection{Contenuti e risorse}

Quali argomenti tratta sempre in un corso per analfabeti? (sono possibili più risposte)

Con questa domanda, che prevede la possibilità di indicare più risposte, si intende indagare quali siano gli ambiti comunicativi che sono sempre affrontati in un corso per studenti analfabeti. Il totale degli informatori (32; 100\%) tratta sempre l'argomento relativo alla «presentazione di sé», come possiamo osservare nella figura 11. 
Quali argomenti tratta sempre in un corso per analfabeti? (sono possibili più risposte) 32 risposte

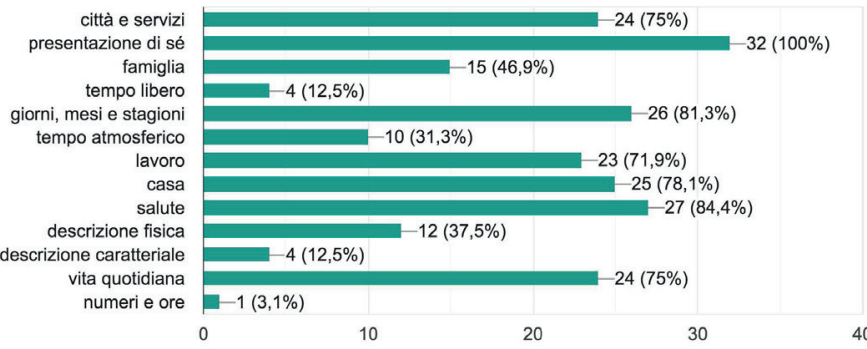

Figura 11 Risposte del questionario: ambiti comunicativi trattati in un corso di L2 con studenti analfabet

Mentre gli altri temi si suddividono, in ordine decrescente, come segue:

- $84,4 \%$ la salute;

- $81,3 \%$ i giorni, i mesi e le stagioni;

- 78,1\% la casa;

- 75\% la città e i servizi e un altro 75\% la vita quotidiana;

- $71,9 \%$ il lavoro;

- $46,9 \%$ la famiglia;

- $37,5 \%$ la descrizione fisica;

- $31,3 \%$ il tempo atmosferico;

- $12,5 \%$ la descrizione del carattere e un altro $12,5 \%$ il tempo libero;

- $3,1 \%$ i numeri e le ore.

Quali sono gli argomenti che, nella sua esperienza, sono più interessanti per gli studenti analfabeti? Ne scriva 4

Con questa domanda, che prevede la possibilità di indicare 4 risposte, si fa leva sull'esperienza personale dei docenti che hanno aderito all'indagine poiché si vuole indagare quali siano stati gli ambiti comunicativi di maggior interesse per corsisti adulti analfabeti.

Gli argomenti che hanno ottenuto un miglior riscontro, secondo un elenco in ordine decrescente, sono:

- le parti del corpo e salute (20 informatori);

- il lavoro (19 informatori);

- la città e i servizi (16 informatori);

- la presentazione di sé (15 informatori);

- la vita quotidiana e la casa (8 informatori);

- la famiglia e il cibo (6 informatori);

- i giorni e le stagioni (3 informatori); 
- la descrizione di sé e degli altri e il tempo libero e lo sport (2 informatori);

- documenti (1 informatore)

Solo un informatore fa una distinzione di genere indicando come, per le donne, gli argomenti più interessanti siano la famiglia, la vita quotidiana e la salute mentre, per gli uomini, il lavoro, la vita quotidiana, la salute e la città e i servizi.

Quali volumi usa in classi di studenti analfabeti?

(sono possibili più risposte)

Tramite questa domanda, che prevede la possibilità di indicare più risposte, si vuole ottenere una panoramica dei volumi per analfabeti più utilizzati per capire se i docenti li conoscano (tutti o in parte), se si costruiscano materiale ad hoc oppure se cerchino altre attività nel web, integrative o sostitutive dei materiali cartacei.

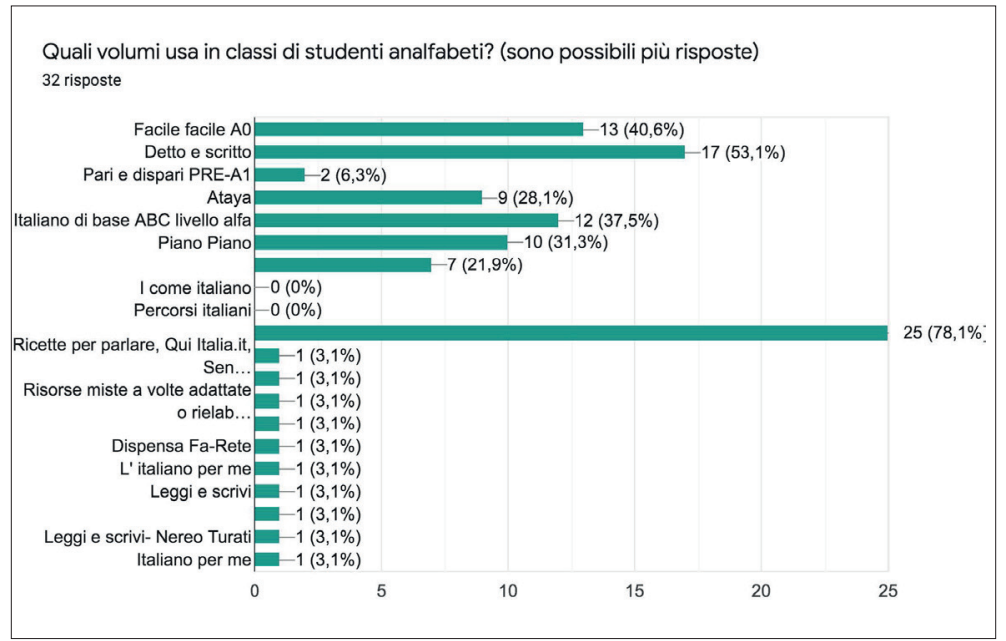

Figura 12 Risposte del questionario: volumi più utilizzati per studenti analfabet

Il 78,1\% degli informatori, come osserviamo nella figura 12, realizza materiale autentico autoprodotto mentre il 53,1\% utilizza il manuale Detto e scritto di Borri e Minuz (2013) e il 40,6\% Facile Facile. A0 di Mattioli e Cassiani (2010).

Il 37,5\% adotta Italiano di base ABC. Livello Alfa di Catanese (2016); il 31,3\% Piano Piano di Rickler e Borio (2019); il 28,1\% Ataya di Aloisi e Perna (2019); il 21,9\% Italiano di base. Livello preA1-A2 di Bol- 
zoni et al. (2016); il 6,3\% Pari e dispari. PreA1 di Borri et al. (2016).

Alla voce «altro» si indicano:

- Leggi e Scrivi - Percorsi e materiali didattici per l'insegnamento della lettura e della scrittura attraverso la lingua italiana ad adulti non madrelingua - volume 1 di Turati (2017) (9,3\%);

- L'italiano per me 1 di Casi (2008) (9,3\%);

- Evviva! Si impara l'italiano di Arrighi (2005) (3,1\%);

- Senza frontiere 1 di Flammini e Pasqualini (2015) (3,1\%);

- Qui Italia.it di Mazzetti e Falcinelli (2011) (3,1\%);

- la dispensa Fa_Rete realizzata dal Comune di Venezia (Brichese, Bortolon 2012) all'interno di un progetto FAMI $(3,1 \%)$;

- Ricette per parlare di Consonno e Bailini (2002) (3,1\%);

- un manuale realizzato dall'Istituto comprensivo Cadeo (3,1\%).

Solo 2 dei 32 informatori indicano come manuale unico Facile Facile A0 mentre 3 affermano di utilizzare unicamente materiale autoprodotto. I restanti 27 informatori utilizzano più manuali contemporaneamente.

\subsection{I metodi di valutazione/testing}

Con le seguenti domande indaghiamo se i docenti propongono agli studenti dei test d'ingresso, in itinere e finali ed eventualmente come si svolgono.

\section{Effettua un test d'ingresso?}

Con questa domanda comprendiamo se i docenti somministrano un test d'ingresso per conoscere le competenze iniziali degli studenti.

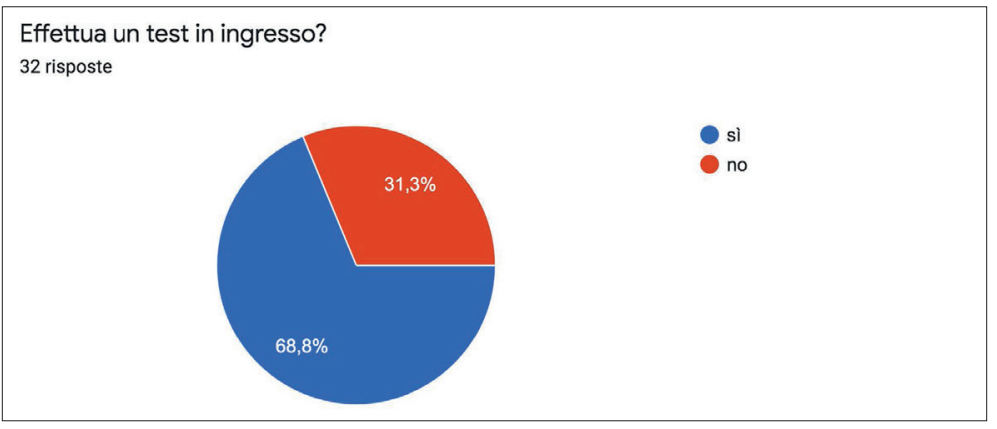

Figura 13 Test d'ingresso 
La maggioranza dei docenti (22; 68,8\%) afferma di somministrare un test d'ingresso agli studenti, invece i restanti 10 (31,3\%) riportano di non realizzarlo.

Se sì, in quale lingua lo somministra? (sono possibili più risposte)

Con questa domanda, che prevede più di una risposta possibile, scopriamo in quale lingua i docenti somministrano il test d'ingresso agli studenti.

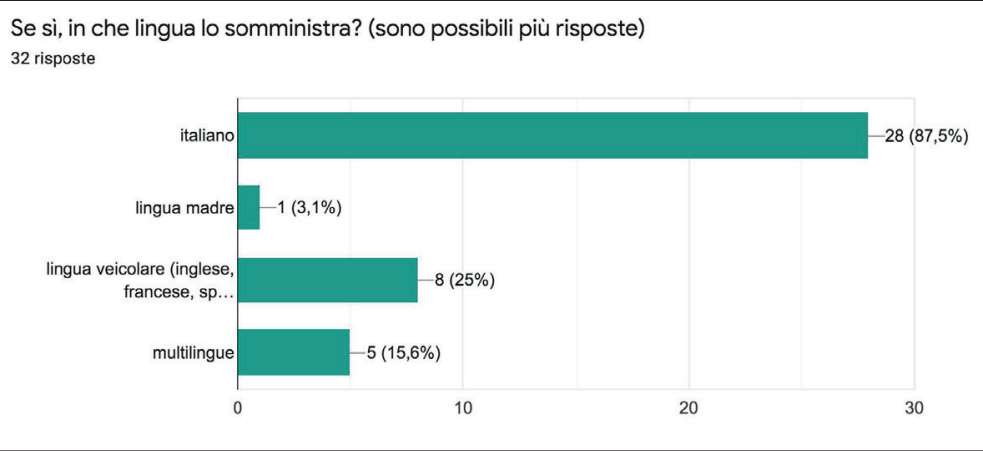

Figura 14 Lingua utilizzata nel test d'ingresso

La quasi totalità dei docenti $(28 ; 87,5 \%)$ propone il test d'ingresso in italiano, 8 docenti (25\%) lo realizzano nella lingua veicolare, 5 docenti $(15,6 \%)$ preferiscono sottoporre agli studenti un test multilingue e solo 1 docente $(3,1 \%)$ lo predispone nella L1 degli studenti.

\section{Quali abilità testa in ingresso? (sono possibili più risposte)}

Con questa domanda, che prevede più di una risposta possibile, esploriamo quali abilità (comprensione orale e scritta, produzione orale e scritta) i docenti testano in ingresso e in quale/i lingua/e avviene tale verifica [fig. 15].

La maggior parte dei docenti (29; 90,6\%) testa le abilità di produzione orale e di interazione orale. Rispettivamente 23 e 20 docenti (71,9\% e $62,5 \%)$ si focalizzano inoltre sulla scrittura e sulla lettura nella lingua italiana. Una minoranza testa la scrittura nella L1 degli studenti $(8 ; 25 \%)$, la scrittura nella lingua veicolare $(7 ; 21,9 \%)$, la produzione orale e l'interazione orale nella lingua veicolare (5; 15,6\%) e la produzione orale e l'interazione orale nella L1 degli studenti (4; 12,5\%). Infine, 3 docenti (9,4\%) verificano l'abilità di lettura nella lin- 


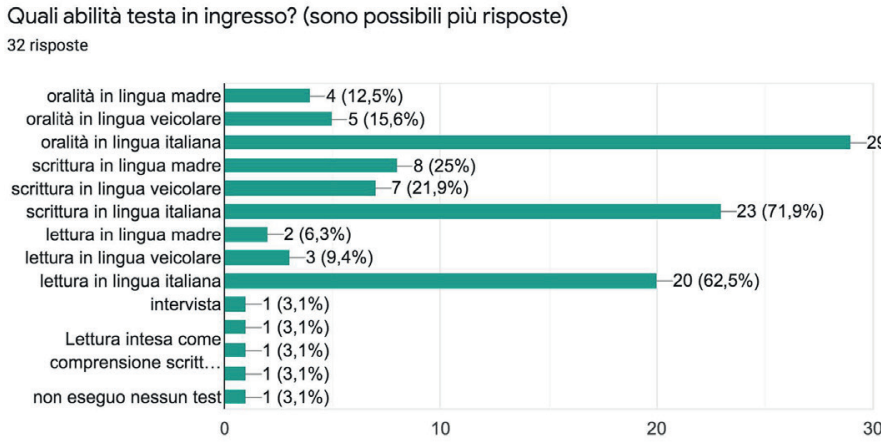

gua veicolare e 2 docenti (6,3\%) si concentrano sull'abilità di lettura nella L1 degli studenti. Alla voce «altro» un docente indica di testare anche il carattere e la motivazione; un docente cerca di capire se gli studenti abbiano delle competenze orali in altre lingue; un docente intervista gli studenti per ricostruire il livello d'istruzione pregressa; un docente svolge un test esclusivamente orale e un docente afferma di non svolgere nessun test.

\section{Effettua un test in itinere?}

Con questa domanda scopriamo se i docenti somministrano uno o più test intermedi per monitorare i progressi degli studenti.

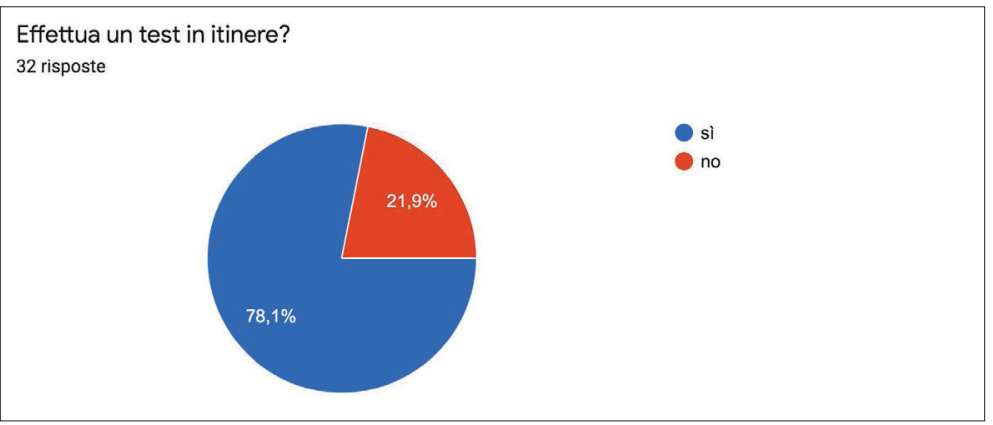

Figura 16 Test in itinere

La maggior parte dei docenti (25; 78,1\%) effettua dei test in itinere, invece i restanti 7 (21,9\%) non li somministrano. 
Effettua un test finale?

Con questa domanda indaghiamo se i docenti somministrano un test finale per verificare il raggiungimento degli obiettivi da parte degli studenti a conclusione del percorso.

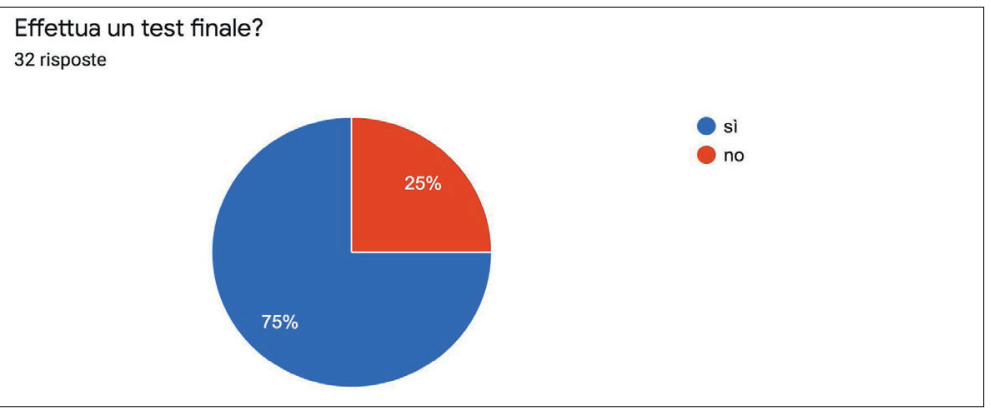

Figura 17 Test finale

La maggior parte dei docenti $(24 ; 75 \%)$ effettua un test di verifica conclusivo, invece i restanti 8 (25\%) non lo somministrano.

\subsection{La gestione del feedback nell'oralità e nella scrittura}

Con le domande di seguito scopriamo qual è l'atteggiamento dei docenti nei confronti degli errori orali e scritti degli studenti e come provvedono a correggerli.

Che cosa fa rispetto agli errori commessi dagli studenti nella produzione orale? (sono possibili più risposte)

Con questa domanda indaghiamo qual è l'atteggiamento dei docenti rispetto agli errori commessi dagli studenti nel corso della produzione orale [fig. 18].

La maggior parte dei docenti $(21 ; 65,6 \%)$ afferma di correggere solo gli errori che compromettono la comprensione del messaggio, 18 docenti $(56,3 \%)$ sostengono di correggere solo gli errori relativi alle parole studiate, 12 docenti (37,5\%) preferiscono chiedere agli studenti di correggere i compagni quando sentono un errore e solo 3 docenti $(9,4 \%)$ riportano di correggere tutti gli errori. Alla voce «altro» un docente scrive di correggere gli errori che si ripresentano più volte e un altro aggiunge di correggere gli errori a patto di non compromettere la fluenza dello studente. Nessuno sostiene di non correggere nessun errore. 


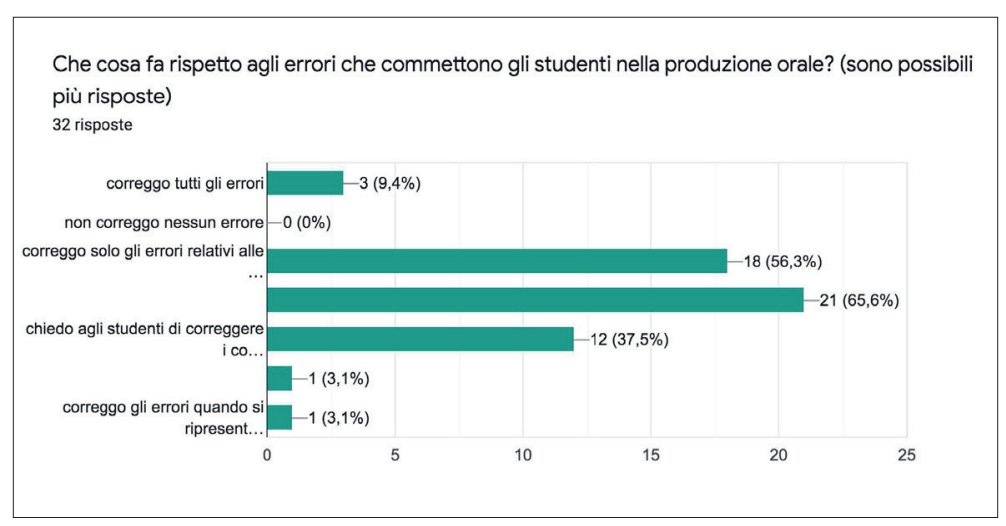

Figura 18 Gestione del feedback nella produzione orale

\section{Come corregge l'errore nella produzione orale?}

Con questa domanda scopriamo come è gestito il feedback dai docenti rispetto agli errori commessi dagli studenti nel corso della produzione orale.

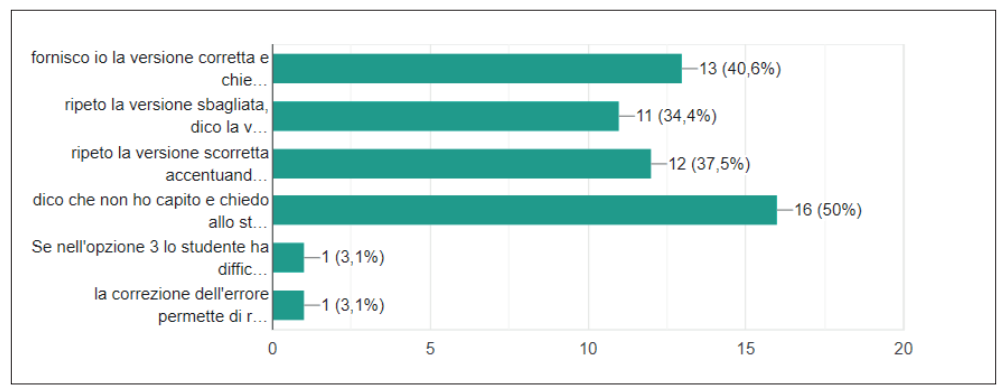

Figura 19 Correzione dell'errore nella produzione orale

Le risposte sono distribuite in maniera piuttosto omogenea. La metà dei docenti $(16 ; 50 \%)$ preferisce comunicare di non aver capito e chiede allo studente di ripetere, 13 docenti $(40,6 \%)$ forniscono la versione corretta e chiedono allo studente di ripeterla, 12 docenti (37,5\%) ripetono la versione scorretta accentuando con la voce solo la parte sbagliata affinché lo studente riesca a correggersi da solo, 11 docenti $(34,4 \%)$ ripetono la versione sbagliata, comunicano la versione corretta e chiedono allo studente di identificare qual è la versione giusta. Alla voce «altro» un docente considera la correzione dell'errore nella produzione orale come un'occasione per ripassare la grammatica e un altro scrive di ricorrere all'aiuto dei compagni. 
Che cosa fa rispetto agli errori commessi dagli studenti nella produzione scritta? (sono possibili più risposte)

Con questa domanda indaghiamo qual è l'atteggiamento dei docenti rispetto agli errori commessi dagli studenti nella produzione scritta.

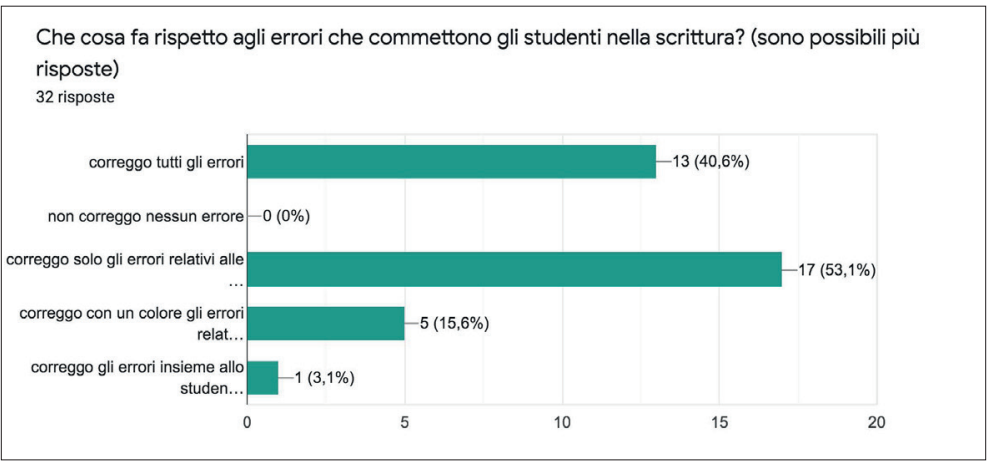

Figura 20 Gestione del feedback nella produzione scritta

Poco più della metà dei docenti $(17 ; 53,1 \%)$ dichiara di correggere solo gli errori relativi alle parole studiate e poco meno della metà (13; $40,6 \%)$ conferma di correggere tutti gli errori. Solo 5 docenti $(15,6 \%)$ ricorrono all'uso dei colori, correggendo con un colore gli errori nelle parole studiate e con un altro quelli nelle parole non studiate. Alla voce «altro» un docente afferma di correggere gli errori assieme allo studente. Nessuno sostiene di non correggere nessun errore [fig. 20].

Come corregge l'errore nella produzione scritta?

(sono possibili più risposte)

Con questa domanda scopriamo come è gestito il feedback dai docenti rispetto agli errori commessi dagli studenti nella produzione scritta [fig. 21].

Le risposte sono suddivise in modo abbastanza omogeneo. Più della metà dei docenti $(17 ; 53,1 \%)$ sostiene sia di leggere quanto scritto dallo studente, dividendo la parola in sillabe e individuando insieme l'errore, sia di fornire allo studente la versione corretta e di chiedere di confrontarla con la propria per trovare e correggere gli errori, copiando infine la versione corretta. Poco meno della metà (14; 43,8\%) afferma di chiedere allo studente di leggere quanto scritto. Solo 7 docenti $(21,9 \%)$ scelgono di segnare gli errori nella produzione dello studente e di invitarlo a correggersi da solo. Alla voce «altro» un docente scrive di leggere quanto scritto dallo studente e di chieder- 


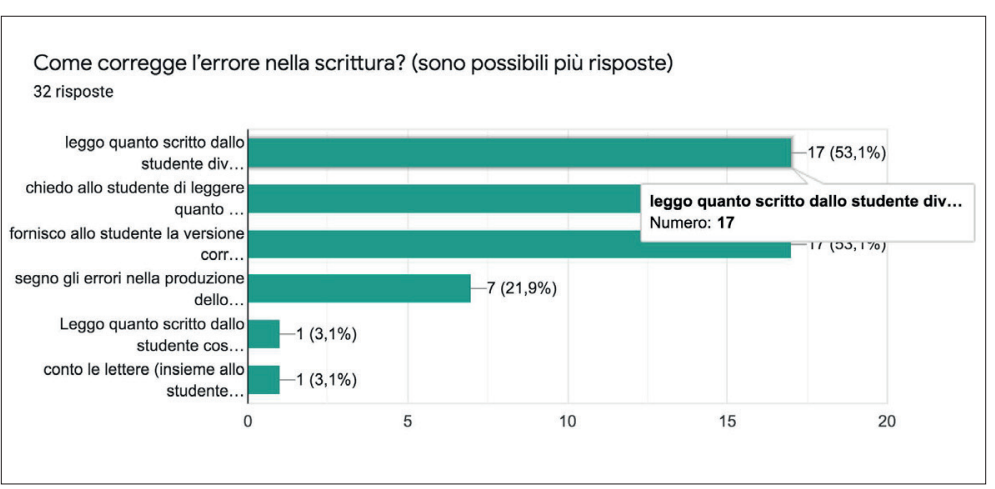

Figura 21 La correzione dell'errore nella produzione scritta

gli di confermare o meno la correttezza, un altro riporta di contare le lettere della parola corretta assieme allo studente per confrontarla con la propria produzione scorretta.

\section{Discussione e conclusioni}

A partire dall'analisi delle risposte scritte dei docenti, riportiamo di seguito la discussione sui risultati e tracciamo delle prime conclusioni che possono rappresentare un utile punto di partenza per l'elaborazione di ulteriori ricerche nell'ambito della didattica a studenti adulti analfabeti in italiano L2.

\subsection{Il profilo del docente}

I dati evidenziano una forte eterogeneità rispetto ai profili dei docenti di italiano L2 che operano all'interno delle realtà dei CAS (nei quali è impiegata la maggioranza degli intervistati), SPRAR e CPIA. Se da un lato la quasi totalità del campione ha risposto affermando di possedere una formazione specifica rispetto all'insegnamento dell'italiano a studenti stranieri, più della metà non ha approfondito, attraverso una formazione $a d$ hoc, le tematiche relative alla didattica per il target analfabeta oggetto della ricerca. Questo aspetto può essere correlato al dato che evidenzia come i 2/3 del campione lavori in questo settore da un massimo di 3 anni.

Inoltre, l'eterogeneità nella formazione, unitamente ad una mancanza di materiali didattici e ad una disformità nei test somministrati, evidenzia la necessità di uniformare il profilo del docente di italiano L2 per adulti stranieri analfabeti in lingua madre, predisponendo percorsi formativi specifici, fornendo indicazioni metodologiche e strumenti operativi per la didattica. 


\subsection{La metodologia didattica}

I dati evidenziano come ci sia ancora molta eterogeneità metodologica nella didattica rivolta a studenti adulti analfabeti.

Infatti, più di $1 / 3$ degli informatori ritiene che questa tipologia di apprendenti necessiti primariamente di esercitare le competenze di letto-scrittura più che le abilità di comprensione e produzione/interazione orali. Inoltre, già all'inizio del percorso di acquisizione linguistica, i 2/3 dei docenti ritiene necessario concentrarsi sugli aspetti della consapevolezza fonologica e della letto-scrittura anziché sull'arricchimento del lessico e sulle abilità in produzione e ricezione orali (restante 1/3 degli intervistati).

Invece, se i $2 / 3$ dei docenti attiva la letto-scrittura a partire da parole note o da materiale autentico precedentemente presentato globalmente, un 1/3 degli intervistati si concentra su singoli fonemi e grafemi, siano essi lettere o sillabe.

Le risposte si diversificano ulteriormente quando si tratta di sondare il metodo utilizzato dai docenti per lavorare sulla lettura e la scrittura.

Per la capacità di decodifica scritta, il 50\% degli intervistati predilige i metodi sintetico-analitici a partire dal lavoro sulle singole lettere o sillabe che poi vanno a comporre le parole, mentre il restante $46,9 \%$ del campione sceglie un approccio di tipo globale al testo per poi arrivare alla scomposizione di singoli suoni contenuti all'interno delle parole.

Per lavorare sulla codifica, invece, i $2 / 3$ del campione inizia con la scrittura di singole lettere o sillabe che vanno poi a comporre la/le parola/e mentre 1/3 degli intervistati propone, al contrario, un approccio che procede dalla scrittura di parole note.

I risultati dello studio di caso su un campione di 32 docenti e facilitatori della provincia di Venezia ha messo in luce la necessità di una formazione specifica sulla metodologia di lavoro con studenti adulti non scolarizzati in L1 poiché, come afferma Colliander:

That the LESLLA teacher teaches initial literacy is one thing that distinguishes her/him from other second language teachers in adult education. According to Williams and Snipper (1990, p. 12), teachers must understand how they conceptualize literacy themselves, in order to be able to develop the students' literacy. This may influence the methods they apply in their teaching and the expected outcomes. (Colliander 2018, 27)

Nell'ambito dell'educazione degli adulti, i docenti che si occupano di alfabetizzazione iniziale si distinguono dagli altri insegnanti di lingua seconda. È fondamentale che chi insegna ad analfabeti e bassamente scolarizzati in L1 definisca prima cosa intende per alfabetizzazione per poter poi sviluppare tale alfabetizzazione degli studenti 
a cui si rivolge. Questa riflessione avrà una conseguente ricaduta nell'applicazione in classe di approcci, metodi e tecniche coerenti col proprio concetto di alfabetizzazione e dei conseguenti risultati attesi.

Come afferma Borri (2019) alfabetizzare non significare solo insegnare una tecnica, ma favorire l'acquisizione di una competenza linguistico-comunicativa per agire nel mondo.

L'alfabetizzazione, dunque, segna l'accesso in una società altamente testualizzata in cui la lettura e la scrittura sono un potente mezzo di produzione, mediazione e circolazione di significati sociali e culturali e a volte sostituiscono le relazioni dirette. Consente inoltre, più nello specifico, l'accesso alla scolarizzazione fondamentale per l'accesso nel mondo del lavoro.

Tale concezione di alfabetizzazione induce a ripensare il concetto di acquisizione strumentale delle abilità di lettura e scrittura non più come sole abilità di codifica e decodifica in quanto esse comportano usi differenziati della lingua scritta in relazione a obiettivi, contesti e interlocutori diversi.

Lo studente deve perciò sviluppare contemporaneamente:

- competenze tecniche di letto-scrittura (ad esempio l'abbinamento grafema e fonema);

- competenze funzionali (ad esempio il sapersi orientare in un volantino di informazioni o leggere l'orario di un avviso);

- competenze socio-culturali e pragmatiche (ad esempio individuare e riconoscere la valenza regolativa di un segnale di divieto o di una parola).

\subsection{Contenuti e volumi}

Vi è un sostanziale accordo tra gli intervistati nel rilevare quali siano gli argomenti imprescindibili di un corso di lingua italiana per questo target di apprendenti, ovvero, tra i principali, le parti del corpo e la salute, il lavoro, la città e i servizi e la presentazione di sé. I docenti e facilitatori sembrano dare importanza al vissuto degli studenti, ai contesti ad essi noti e familiari e alla spendibilità immediata in contesto autentico e quotidiano di quanto appreso in classe.

Sembra essere, invece, prassi consolidata la costruzione da parte dei docenti di attività ad hoc e l'integrazione di diversi materiali estrapolati da più manuali presenti in commercio. Il dato ci dice che l'offerta didattica in commercio rivolta a questo specifico target di apprendenti non soddisfa pienamente le esigenze dei docenti che sono costretti, perciò, a realizzare da sé le attività oppure a reperire materiale dalla rete 0 a creare dei collage di attività diverse ripresa da più manuali.

Si sottolinea, inoltre, che fra i manuali elencati solo $1 / 4$ si rivolge, nello specifico, al target adulto analfabeta. 
Molti dei manuali indicati, infatti, non sono dedicati a classi composte da studenti analfabeti in lingua madre. Si vedano, ad esempio:

a. Italiano di base. Livello PreA1-A2 che in apertura dichiara di essere «un corso di lingua italiana finalizzato ad accompagnare studenti migranti nel processo di integrazione linguistico-culturale previsto dalla normativa vigente. Si rivolge a studenti che presentano in ingresso un livello preA1 ${ }^{6}$ o A1 e che intendono raggiungere i livelli A1 e A2 descritti dal Quadro comune Europeo di Riferimento per le lingue» (Bolzoni et al. 2016, 3);

b. Evviva! Si impara l'italiano di Arrighi (2005) invece è un percorso di apprendimento attivo della lingua italiana per l'Intercultura per alunni stranieri inseriti nelle ultime due classi della scuola elementare e nelle tre classi della scuola media, nonché frequentanti i Centri Territoriali non specifico per il target adulto analfabeta in L1;

c. Senza frontiere 1. A1 di Flammini e Pasqualini (2015) è indicato come un manuale con materiali didattici di qualità per chi deve insegnare ad immigrati. Il corso è composto da due volumi per i livelli A1-A2;

d. Qui Italia.it di Mazzetti, Falcinelli, Servadio, Santeusanio (2011) è un corso di apprendimento della lingua italiana per stranieri di livello elementare, A1 e A2;

e. Ricette per parlare di Consonno e Bailini (nella versione aggiornata, 2017) contiene attività per la produzione orale libera o guidata, dal livello principiante (A1) al livello avanzato (C1), da realizzare a coppie o in gruppo, basate su varie tecniche didattiche;

f. Facile Facile A0. Livello Principianti Assoluti di Mattioli e Cassiani (2010) è nato dopo i volumi A1 e A2, ma li precede nel percorso di acquisizione della lingua italiana a livello elementare. Nasce per rispondere all'esigenza, riscontrata 'sul cam$\mathrm{po}^{\prime}$, di fornire una guida ancor più semplice e graduale, rispetto al livello A1, per coloro che hanno difficoltà ad entrare in un processo di apprendimento rapido della nostra lingua.

6 In nota gli autori specificano che «il QCER non prevede descrittori di competenza preliminari al livello A1; adottando la dicitura preA1, questo testo indica un livello Pre-QCER, caratteristico di apprendenti alfabetizzati ma che non hanno alcuna o solo minima competenza in lingua italiana» (Bolzoni et al. 2016, 3). In realtà, nel 2018 è uscito il Compendio al Quadro (Companion Volume with New Descriptors 2018) in cui è inserito un nuovo livello, il pre-A1, che precede il livello A1 nel continuum dell'acquisizione linguistica in lingua seconda. 


\subsection{I metodi di valutazione/testing}

Dalla considerazione dei dati relativi ai metodi di valutazione/testing emerge che la maggior parte dei docenti effettua dei test d'ingresso $(22 ; 68,8 \%)$, in itinere $(25 ; 78,1 \%)$ e finali $(24 ; 75 \%)$ per valutare le preconoscenze degli studenti, monitorare i progressi e verificare il raggiungimento degli obiettivi didattici.

Riguardo al test d'ingresso, dai risultati si rileva che una quota significativa dei docenti $(28 ; 87,5 \%)$ lo somministra in italiano, invece solo un docente $(3,1 \%)$ lo propone nella L1 degli studenti. A nostro avviso, questo dato è significativo poiché un test in italiano può indicare se gli studenti sono alfabetizzati nei caratteri latini, ma non determina se e quanto essi siano scolarizzati nella L1. Pertanto, per determinare se uno studente è analfabeta nella propria L1 il test dovrebbe essere somministrato nella lingua madre o, se conosciuta, nella lingua veicolare. Inoltre, dai risultati si evince che la quasi totalità del campione $(29 ; 90,6 \%)$ si concentra sulla valutazione delle competenze nella produzione orale e nell'interazione orale, necessaria per ricostruire il background degli studenti e definirne il profilo linguistico. Questo è nuovamente possibile quando i test in ingresso sono svolti nella lingua madre o, se conosciuta, nella lingua veicolare.

Dai dati emerge una grande eterogeneità rispetto alla lingua in cui somministrare i test d'ingresso e alle abilità da testare. In effetti, ad oggi gli strumenti validati scientificamente per sondare le competenze pregresse degli studenti, capire se sono scolarizzati nella lingua madre e identificare quale livello hanno raggiunto risultano ancora scarsi. Una proposta di testing in ingresso per i profili dell'analfabeta totale e funzionale, in corso di validazione, è elaborata da Rocca (2019).

\subsection{La gestione del feedback nell'oralità e nella scrittura}

Dalle seppur esigue indagini riguardanti l'efficacia delle principali strategie di gestione del feedback nell'oralità e nella scrittura (Mackey, Oliver 2002; Sheen 2004; Lyster, Saito 2010; Grassi 2012) è possibile affermare che essa cambi a seconda del contesto didattico, dell'esperienza scolastica pregressa, dell'età e della tipologia di corso. D’altra parte, negli ultimi decenni sono state realizzate molteplici indagini sulle modalità di insegnamento dei facilitatori ad apprendenti adulti analfabeti e debolmente scolarizzati per evidenziare le competenze didattiche necessarie per poter intervenire adeguatamente (Comings, Soricone 2005; Faux 2006; Kreeft Peyton, Schaetzel, Burt 2007; Mathews-Aydinli 2008; Vinogradov 2009).

Rispetto alla gestione del feedback nell'oralità, oltre alla scelta di intervenire nel caso di fraintendimenti comunicativi o di in- 
certezze sui contenuti già affrontati, si sostiene l'auto-correzione tra compagni, incentivando così il tutoraggio tra pari. Particolarmente rappresentativo appare quanto scritto alla voce «altro» da uno dei docenti:

in genere cerco di correggere la maggior parte degli errori se questo non compromette la fluidità del discorso. Spesso chiedo l'aiuto dei compagni a seconda dell'attività. Solo in momenti di interazione semi-informale correggo solo gli errori che compromettono la comprensione del messaggio.

Si ritiene fondamentale specificare che il ricorso a metodologie a mediazione sociale, soprattutto con questa tipologia di apprendenti, richiede un'adeguata conoscenza tra compagni e dunque può risultare efficace se all'interno del gruppo classe si è sviluppata una buona armonia (Caon 2016).

I docenti impiegano una grande varietà di strategie per la gestione del feedback nell'oralità, tra cui la richiesta di chiarimento e la ripetizione, suggerite soprattutto con apprendenti adulti debolmente scolarizzati, e la riformulazione e la ripetizione 'per contrasto', indicate specialmente con apprendenti adulti analfabeti (Spaliviero 2019). Con la richiesta di chiarimento si chiede allo studente di ripetere o di riformulare quanto detto, invece con la ripetizione si riporta la produzione scorretta dello studente accentuando con la voce solo la parte sbagliata affinché lo studente riesca a correggerla da solo. Con la riformulazione si rielabora la produzione errata dello studente fornendo la versione corretta e si chiede allo studente di pronunciarla di nuovo, invece con la ripetizione 'per contrasto' si riporta la produzione scorretta dello studente e si propone anche la versione corretta, chiedendo allo studente di indicare l'opzione giusta per sensibilizzarlo al riconoscimento dei suoni. Anche se con minore frequenza, è indicato nuovamente il ricorso ai compagni nel ruolo di tutor. Scrive infatti un docente alla voce «altro»:

se nell'opzione 3 [ripeto la versione scorretta accentuando con la voce solo la parte sbagliata affinché lo studente riesca a correggerla da solo] lo studente ha difficoltà, per non indurre stress fornisco e/o faccio fornire la versione corretta dai compagni, accentuando il tono là dove 'cade' la parte corretta.

Riguardo alla gestione del feedback nella scrittura, la maggior parte dei docenti interviene per correggere l'errore solo se quest'ultimo riguarda le parole-obiettivo già affrontate. Con questa tipologia di studenti nello specifico, la necessità di selezionare quali errori sia prioritario correggere è connessa alla complessità dell'avviamento del percorso di scrittura e alle numerose abilità da possedere. 
Anche in questo caso, i docenti utilizzano molteplici strategie, come la correzione risolutiva, quella rilevativa e la lettura di quanto scritto dagli studenti con la suddivisione in sillabe per favorire l'individuazione dell'errore, dimostrando di favorire sia l'etero-correzione sia l'auto-correzione (Spaliviero 2019). Con la correzione risolutiva si fornisce allo studente la versione corretta della parola sbagliata e gli si chiede di confrontarla con la propria per capire cosa deve essere modificato, invece con la correzione rilevativa si segna l'errore senza fornire la versione corretta e si chiede allo studente di riflettere sull'errore e di correggersi da solo. La lettura e la suddivisione in sillabe di quanto scritto può realizzarsi con la procedura delle 'lettere in tabella', tale per cui si crea una tabella con tanti quadretti in orizzontale e in verticale quante sono le lettere della parola e si guida lo studente dal piano semantico a quello ortografico, facendolo riflettere anche a livello fonologico e fonetico. Riteniamo necessario sottolineare che queste strategie, ben differenziate nella definizione teorica, possono essere integrate nella prassi didattica a seconda della tipologia di attività e di apprendente. Scrive al riguardo un docente alla voce «altro»:

conto le lettere (insieme allo studente, se in grado di farlo) della parola corretta, scandisco fonema per fonema, conto le lettere della parola scorretta e poi confronto (assieme allo studente) le due versioni.

\section{Bibliografia}

Aloisi, E.; Perna, A. (2019). Ataya. Bergamo: Sestante Edizioni.

Arrighi, E. (2005). Evviva! S'impara l'italiano. Brescia: Vannini Editore.

Benítez, M. Elena; Borzone, A.M. (2012). «Estrategias de lectura y escritura en jóvenes y adultos con bajo nivel de alfabetización». Revista del Instituto de Investigaciones en Ciencias de la Educación, 31, 117-34.

Benseman, J. (2012). Adult Refugee Learners with Limited Literacy. Needs and Effective Responses. Wellington: English Language Partners.

Bigelow, M.; Schwarz, R.L. (2010). Adult English Language Learners with Limited Literacy. Washington, DC: National Institute for Literacy.

Bolzoni, A. et al. (2016). Italiano di base. Livello preA1-A2. Firenze: Alma Edizioni.

Boon, D. (2014). Adult Literacy Education in a Multilingual Context Teaching, Learning and Using Written Language in Timor-Leste. Tilburg: Tilburg University.

Borri, A. (2019). «Gli approcci e i metodi nell'alfabetizzazione degli adulti». Caon, Brichese 2019, 89-97.

Borri, A. et al. (2014). Italiano L2 in contesti migratori. Sillabo e descrittori dall'alfabetizzazione all'A1. Torino: Loescher.

Borri, A. et al. (2016). Pari e dispari preA1. Torino: Loescher.

Borri, A.; Minuz, F. (2013). Detto e scritto. Torino: Loescher. 
Borri, A.; Minuz, F. (2016). «Literacy and Language Teaching. Tools, Implementation and Impact». ItalianoLinguaDue, 2, 220-31. https://doi. org/10.13130/2037-3597/8184.

Brichese, A.; Bortolon, G. (a cura di) (2012). Fa_Rete. https://bit.ly/3jFt5Pw.

Burt, M.; Kreeft Peyton, J.; Schaetzel, K. (2008). «Working with Adult English Language Learners with Limited Literacy. Research, Practice, and Professional Development». Washington, DC: Center for Applied Linguistics. Caela NetworkBrief. http://www.cal.org/adultesl/pdfs/working-withadult-english-language-learners-with-limited-literacy.pdf.

Calcagno, S. et al. (2016). «Análise dos erros apresentados por adultos iletrados ao longo de um programa informatizado de ensino de leitura e escrita». Acta Colombiana de Psicología, 19(1), 137-50.

Caon, F. (a cura di) (2016). Educazione linguistica nella classe ad abilità differenziate. Torino: Loescher; Bonacci.

Caon, F.; Brichese, A. (a cura di) (2019). Insegnare l'italiano ad analfabeti. Torino: Loescher; Bonacci.

Cardoso-Martins, C.; Rodrigues, L.A.; Ehri, L.C. (2003). «Place of Environmental Print in Reading Development: Evidence From Nonliterate Adults». Scientific Studies of Reading, 7(4), 335-55. https://doi.org/10.1207/ S1532799XSSR0704_2.

Casi, P. (2008). L'italiano per me 1. Ancona: ELI.

Catanese, P. (2016). Italiano di base ABC. Livello Alfa. Firenze: Alma Edizioni.

Choi, J.; Ziegler, G. (2015). «Literacy Education for Low-Educated Second Language Learning Adults in Multilingual Contexts. The Case of Luxembourg». Multilingual Education, 5(1). https://doi.org/10.1186/s13616-@150024-7.

Colliander, H. (2018). Being and Becoming a Teacher in Initial Literacy and Second Language Education for Adults. Linköping: Linköping University.

Comings, J.P.; Soricone, L. (2005). The World Education Approach to Adult Literacy Program Design. Boston (MA): World Education.

Consiglio d'Europa (a cura di) (2017). Supporto linguistico per rifugiati adulti: il Toolkit. Strasburgo: Consiglio d'Europa. https://ec.europa.eu/epale/ it/resource-centre/content/toolkit-del-consiglio-deuropa.

Consonno, S.; Bailini, S. (2002). Ricette per parlare. Firenze: Alma.

Council of Europe (ed.) (2018). Common European Framework of Reference for Languages. Learning, Teaching, Assessment. Companion Volume with New Descriptors. Strasbourg: Council of Europe. https://www.coe.int/en/ web/common-european-framework-reference-languages.

Creswell, J.W. (2009). Research Design. Qualitative, Quantitative and Mixed Methods Approaches. Thousand Oaks; London; New Delhi: Sage.

Cronen, S.; Silver-Pacuilla, H.; Condelli, L. (2011). Conducting Large-Scale Research in Adult ESL. Challenges and Approaches for the Explicit Literacy Impact Study. Washington, DC: American Institute for Research.

Elmeroth, E. (2010). «From Refugee Camp to Solitary Confinement. IIliterate Adults Learn Swedish as a Second Language». Scandinavian Journal of Educational Research, 47(4), 431-49. https://doi. org/10.1080/00313830308593.

Eme, E. (2011). «Cognitive and Psycholinguistic Skills of Adults Who Are Illiterate. Current State of Research and Implications for Adult Education». Applied Cognitive Psychology, 25(5), 753-62. 
Faux, N.R. (2006). «Preparing Teachers to Help Low-Literacy Adult ESOL Learners». Van de Craats, J.; Kurvers, J.; Young-Scholten, M. (eds), Low-Educated Adult Second Language and Literacy Acquisition. Proceedings of the Inaugural Symposium. Utrecht: LOT, 135-42.

Faux, N.R. (ed.) (2007). Low-Educated Second Language and Literacy Acquisition. Research, Policy and Practice. Proceedings of the Second Annual Forum. Richmond (VA): The Literacy Institute at Virginia Commonwealth University.

Flammini, P.; Pasqualini, T. (2015). Senza frontiere 1. Atene: Edilingua.

Flick, U. (2014). An Introduction to Qualitative Research. Thousand Oaks; London; New Delhi: Sage.

Fulanete Corrêa, M.; Cardoso-Martins, C.; Rodrigues, L.A. (2010). «O conhecimento do nome das letras e a sua relação com o desenvolvimento da escrita: evidência de adultos iletrados». Psicologia. Reflexão e Crítica, 23(1), 161-5. https://doi.org/10.1590/S0102-79722010000100019.

Grassi, R. (2012). «ll 'peso' del contesto nell'apprendimento dell'italiano come lingua seconda». Grassi, R. (a cura di), Nuovi contesti d'acquisizione e insegnamento: l'italiano nelle realtà plurilingui. Perugia: Guerra, 15-34.

Kern, R.; Schultz, J.M. (2005).«Beyond Orality, Investigating Literacy and the Literary in Second and Foreign Language Instruction». The Modern Language Journal, 89(3), 381-92. https://doi.org/10.1111/j.15404781.2005.00312.x.

Knowles, M.S. et al. (1998). The Adult Learner. The Definitive Classic in Adult Education and Human Resource Development. Houston (TX): Gulf Publishing Company.

Kreeft Peyton, J.; Schaetzel, K.; Burt, M. (2007). «Professional Development for Practitioners Working with Adult English Language Learners with Limited Literacy». Faux 2007, 213-25.

Kurvers, J. (2007). «Development of Word Recognition Skills of Adult L2 Beginning Readers». Faux 2007, 23-43.

Lyster, R.; Saito, K. (2010). «Interactional Feedback as Instructional Input. A Synthesis of Classroom SLA Research». Language, Interaction and Acquisition, 1(2), 276-97. https://doi.org/10.1075/lia.1.2.07lys.

Machetti, S.; Rocca, L. (2017). «Integration of Migrants, from Language Proficiency to Knowledge of Society. The Italian Case». Beacco, J-C. et al. (eds), The Linguistic Integration of Adult Migrants. L'intégration linguistique des migrants adultes. Strasbourg: Council of Europe, 213-18. https://doi. org/10.1515/9783110477498.

Mackey, A.; Oliver, R. (2002). «Interactional Feedback and Children's L2 Development». System, 30(4), 459-77. https://doi.org/10.1016/s0346$251 \times(02) \odot 0049-\odot$.

Marrapodi, J. (2013). «What Doesn't Work for the Lowest Level Literacy Learners and Why?». Apples. Journal of Applied Language Studies, 7(1), 7-23.

Martín Fernández, P. (2013). «Alfabetizando arabófonos adultos. Un estudio de casos». Revista Nebrija de Lingüística Aplicada, 13. https://www.nebrija.com/revista-linguistica/alfabetizando-arabofonos-adultos.html.

Mathews-Aydinli, J. (2008). «Overlooked and Understudied? A Survey of Current Trendsin Research on AdultEnglish Language Learners».AdultEducation Quarterly, 58(3), 198-213. https://doi.org/10.1177\%2F0741713608314089.

Mattioli, L.; Cassiani, P. (2010). Facile Facile A0. Pesaro: Nina Edizioni.

Mazzetti, A.; Falcinelli, M. (2011). Qui Italia.it. Milano: Mondadori. 
Rickler, P.; Borio, M. (2017). Piano Piano. Milano: Guerini e Associati.

Rocca, L. (2019). «La gestione della fase di accoglienza dell'utenza vulnerabile: intervista e test». Caon, Brichese 2019, 69-87.

Shapiro, S.; Farrelly, R.; Curry, M.J. (2018). Educating Refugee-Background Students. Critical Issues and Dynamic Contexts. Bristol: Multilingual Matters.

Sheen, Y.Hee (2004). "Corrective Feedback and Learner Uptake in Communicative Classrooms across Institutional Settings». Language Teaching Research, 8(3), 263-300. https://doi.org/10.1191/1362168804lr146oa.

Spaliviero, C. (2019). «La gestione del feedback con apprendenti adulti analfabeti e debolmente scolarizzati». Caon, Brichese 2019, 183-9.

SPRAR (2015). Manuale operativo per l'attivazione e la gestione di servizi di accoglienza integrata in favore di richiedenti e titolari di protezione internazionale e umanitaria. Servizio centrale del Sistema di protezione per richiedenti asilo e rifugiati, Ministero dell'Interno. https://www.siproimi. it/wp-content/uploads/2016/06/SPRAR_-_Manuale_operativo_2015.pdf.

Stake, R. (1995). The Art of Case Study Research. London; Thousand Oaks; New Delhi: Sage.

Tammelin-Laine, T.; Martin, M. (2014). «The Simultaneous Development of Receptive Skills in an Orthographically Transparent Second Language». Writing Systems Research, 7(1), 39-57. https://doi.org/10.1080/17586801 .2014 .943148

Tarone, E.; Bigelow, M.; Hansen, K. (2009). Literacy and Second Language Oracy. Oxford: Oxford University Press.

Turati, N. (2017). Leggi e scrivi. Vicenza: CPIA Vicenza.

Villa Carpio Fernández, M.; Defior Citoler, S.A.; Justicia Justicia, F. (2002). «Habilidades fonológicas y lectura en adultos analfabetos». Revista galego-portuguesa de psicoloxía e educación. Revista de estudios e investigación en psicología y educación, 8(6), 179-218.

Vinogradov, P. (2009). Principled Preparation for Teachers of Adult Low-Literate Learners. Paper presented at the Sixth International Language Teacher Education Conference. Washington, DC: George Washington University.

Vinogradov, P. (2013). Professional Learning across Contexts for LESLLA Teachers. The Unlikely Meeting of Adult Educators in Kindergarten to Explore Early Literacy Instruction [PhD Dissertation]. Minneapolis: University of Minnesota.

Vinogradov, P.; Bigelow, M. (2010). «Using Oral Language Skills to Build on the Emerging Literacy of Adult English Learners». Washington, DC: Center for Applied Linguistics. Caela Network Brief. http://www.cal.org/adultesl/pdfs/briefs/using-oral-language-skills.pdf.

Zimmari, E. (2014). «Strategie didattiche e interventi d'aula per l'insegnamento ad adulti stranieri». Bollettinoltals, 55. https://www.itals.it/strategie-didattiche-e-interventi-d\%E2\%80\%99aula-l\%E2\%80\%99insegnamento-ad-adulti-stranieri. 


\title{
EL.LE
}

Vol. 9 - Num. 1 - Marzo 2020

\section{Apprendimento dell'italiano in Camerun Comprendere e usare le espressioni idiomatiche e altre locuzioni fisse}

\author{
Vicky Mazoua Megni Tchio \\ Università Ca' Foscari Venezia, Italia
}

\begin{abstract}
Linguistic research has demonstrated the importance of theories of language interference and positive or negative transfers from one language to another. Our study is based on written texts produced by the Italian language students of the ENS of the University of Maroua. The observed data indicated a clear influence of French and especially of its local variants over the use of idiomatic expressions. It is therefore legitimate to ask whether the lexical and syntactic deviations of French-speaking learners of Italian as a foreign language in Cameroon are only the result of a closeness of the two linguistic systems, or of an inadequate competence in the French language. The purpose of our work is to analyze the causes of such interference and possibly to draw useful strategies for the teaching of Italian in Cameroon.
\end{abstract}

Keywords Italian as foreign language. Idiomatic expressions. Interferences. Figurative competence.

Sommario 1 Introduzione. - 2 Tipologie e caratteristiche delle forme idiomatiche. 3 Espressioni idiomatiche e insegnamento/apprendimento dell'italiano LS. - 4 Contesto e strumenti di studio. - 5 Risultati. - 5.1 Problemi di comprensione e di traduzione delle espressioni idiomatiche. - 5.2 Problemi nella produzione delle espressioni idiomatiche. - 6 Proposte didattiche per l'apprendimento delle espressioni idiomatiche in italiano LS.

\section{Peer review}

Submitted 2019-01-09

Accepted 2020-04-23

Published 2020-07-30

\section{Open access}

(C) 2020 | (a) Creative Commons Attribution 4.0 International Public License 


\section{Introduzione}

Il contatto tra lingue dà luogo a fenomeni linguistici come l'interferenza, una sovrapposizione di diversi codici nella produzione linguistica individuale. Quando il contatto avviene tra lingue imparentate, la L1 sembra avere un'influenza maggiore rispetto al caso di lingue tipologicamente molto distanti. La grande somiglianza a livello strutturale dell'italiano e del francese, che hanno conservato molti tratti comuni dalla loro origine latina, agevola spesso la comprensione e la produzione da parte di apprendenti francofoni dell'italiano, ma può anche essere causa di transfer negativi.

Molte ricerche sono state condotte sulle interferenze e i transfer positivi e negativi degli apprendenti francofoni dell'italiano (Covino Bisaccia 1996; Spreafico 2006; Kuitche Talé 2013), alcune delle quali hanno preso in esame un campione specifico di apprendenti camerunesi francofoni. Una particolare specificità di questo campione è proprio il fatto che gli apprendenti abbiano in comune non solo la L1, ma anche uno stesso background sociolinguistico e culturale. Queste ricerche hanno prevalentemente fatto una rassegna degli errori più frequenti dei francofoni nelle produzioni orali o scritte in lingua italiana, cercando di capire quali fossero i motivi della ricorrenza di tali errori, ma anche quali strategie operative mettere in atto per prevederli e conseguentemente evitarli.

La nostra riflessione nasce dalla constatazione che l'acquisizione e il successivo riutilizzo del linguaggio figurato non va di pari passo con le altre strutture linguistiche dell'italiano. In questo contributo ci interessiamo più specificatamente a come gli apprendenti camerunesi francofoni percepiscono e traducono le espressioni fisse dell'italiano e come le realizzano nelle loro produzioni scritte. In effetti, la presenza massiccia di queste espressioni nella comunicazione quotidiana dimostra la loro importanza per la conoscenza del lessico complessivo della lingua italiana e quindi anche per l'acquisizione della competenza linguistico-comunicativa.

Il corpus analizzato è composto principalmente da composizioni scritte su argomenti vari, raccolte durante tre semestri universitari presso un gruppo di studenti di italiano della Scuola Normale Superiore di Maroua ${ }^{1}$ in Camerun. Oltre alle composizioni scritte, le letture in classe nell'ambito delle esercitazioni previste durante le lezioni di traduzione e le attività del laboratorio di lingua italiana hanno permesso di raccogliere alcune delle espressioni analizzate nel presente articolo. I dati esaminati indicano una netta influenza del francese - e

1 La Scuola Normale Superiore dell’Università di Maroua è dal 2008 l'unica scuola professionale in Camerun dedicata alla formazione pedagogica e didattica degli aspiranti insegnanti di italiano per le scuole secondarie. 
soprattutto della sua variante locale - nell'impiego delle espressioni idiomatiche e altre locuzioni fisse dell'italiano. Si cercherà nel corso di questa trattazione di verificare se le devianze lessicali e strutturali dipendono solo dalla vicinanza tipologica del francese e dell'italiano oppure se sono frutto di una scarsa competenza nella lingua/ cultura di arrivo. Lo scopo di questo contributo è analizzare le cause di tale transfer negativo ed eventualmente trarne delle strategie didattiche utili per il nostro contesto di insegnamento.

\section{Tipologie e caratteristiche delle forme idiomatiche}

L'espressione idiomatica viene definita come una sequenza di parole il cui significato non è dato dalla somma dei significati delle parole che la compongono. Sono considerate forme idiomatiche le espressioni fisse, le frasi fatte, gli idioms, le locuzioni, alcuni modi di dire, ecc. Tutto sommato sono frasi tipiche di una lingua che appartengono al dominio della fraseologia. Queste sequenze fraseologiche sono caratterizzate da una particolare autonomia dal punto di vista lessicale e sintattico e sono difficilmente interpretabili proprio per la loro fissità semantica e strutturale. Perrin afferma a questo proposito che

la notion d'expression idiomatique renvoie à l'ensemble des idiotismes d'une langue, à l'ensemble des locutions perçues comme figées par les usages de cette langue. (Perrin 2003, 281)

La nozione di espressione idiomatica si riferisce all'insieme degli idiotismi di una lingua, all'insieme di locuzioni percepite come fisse dagli usi di tale lingua. (trad. dell'Autore)

Le espressioni idiomatiche sono molto usate nelle interazioni quotidiane. Se per un parlante nativo possono essere trasparenti e di facile interpretazione, per i parlanti non nativi sono spesso incomprensibili e difficilmente accessibili proprio perché il significato della somma delle parole che le costituiscono viene disatteso. In questo caso, per poter decodificare i modi di dire dell'italiano l'apprendente straniero ha bisogno di andare oltre il sistema linguistico e considerare anche gli aspetti culturali e i contesti referenziali. Casadei (2007) sostiene a questo proposito che l'interpretazione di un'espressione idiomatica in un'altra lingua richiede l'intervento di conoscenze aggiuntive relative alla cultura, oltre a quelle relative al vocabolario e alla grammatica di quella lingua. Comprendere gli idiotismi significa quindi addentrarsi profondamente nella conoscenza di una lingua e della relativa cultura.

Le espressioni idiomatiche sono caratterizzate da autonomia lessicale, sintattica e semantica. La loro particolarità risiede nel fatto 
che esse possiedono un significato convenzionale generalmente diverso da quello letterale, sono il prodotto di una convenzione sociolinguistica o di una forma linguistica generalmente e in gran parte fissa, con uno specifico significato condiviso tra i membri di una stessa collettività. Tali espressioni, se a volte sono flessibili, sono principalmente caratterizzate da diversi gradi di opacità che determinano il livello di idiomaticità e perciò anche la distanza dal loro valore semantico letterale.

Nel lessico dell'italiano esistono diverse tipologie di unità lessicali più o meno fisse a cui corrisponde un grado diverso di idiomaticità. Ad esempio, le collocazioni presentano una diversa valenza idiomatica rispetto ad altre espressioni fisse come le formule routinizzate che Lewis (1993) chiama i chunks lessicali. Le collocazioni sono delle unità lessicali in cui a un termine ' $x$ ' corrisponde un termine ' $y$ ' in modo lessicalmente determinato. In base all'uso consolidato all'interno della comunità di parlanti, nella collocazione 'capelli castani', l'aggettivo 'castano' è lessicalmente collegato a parti del corpo umano, 'occhi' e 'capelli' e, sempre nell'uso comune, non si adeguerebbe ad esempio a 'vestito' o 'scarpe'; mentre un'altra collocazione come 'patire la fame' consente la sostituzione di una delle sue componenti ('soffrire la fame') senza alterare il significato dell'espressione. I chunks lessicali invece sono, nel parlato, delle espressioni di routine del linguaggio quotidiano con una forte valenza pragmatica ('fatti vivo', 'quanto tempo', 'va tutto bene', 'facciamo quattro chiacchiere', ecc.) e nello scritto sono le espressioni usate per la coerenza e la coesione del testo quali 'in primo luogo...', 'a partire da...', ecc.

Secondo la classifica di Glucksberg (1993), ripreso da Cardona (2008), esistono due principali categorie di espressioni idiomatiche: la prima è costituita da espressioni il cui significato è dato in modo arbitrario ed è condiviso dalla comunità di parlanti e conservate nella memoria semantica come singole unità ('essere in gamba', 'ingoiare il rospo', 'perdere le staffe') e la seconda è costituita da espressioni che traggono origine da fatti storicamente accaduti e diventati casi esemplari in determinate situazioni come ad esempio 'una vittoria di Pirro', 'venire a Canossa' o 'fare i portoghesi'. In questo contributo ci occuperemo esclusivamente di esempi appartenenti alla prima categoria che, sotto il profilo glottodidattico, presentano difficoltà di natura diversa rispetto a quelli della seconda categoria, soprattutto in modalità LS. ${ }^{2}$

2 In effetti, una delle difficoltà dell'apprendimento di una lingua straniera fuori contesto è proprio il fatto che gli apprendenti non sono sempre a contatto con la storia, la cultura e la civiltà del Paese di cui studiano la lingua. 
Vicky Mazoua Megni Tchio

Apprendimento dell'italiano in Camerun

\section{Espressioni idiomatiche $\mathrm{e}$ insegnamento/apprendimento dell'italiano LS}

La lingua italiana è idiomatica quanto qualsiasi altra. (Porcelli 2004, 55)

Da quanto presentato nel paragrafo precedente, per la loro stessa natura, le espressioni idiomatiche presentano alcune difficoltà nel loro apprendimento. Sono strutture fisse che non rispettano sempre la struttura sintattica dell'italiano e acquisiscono il loro significato proprio dall'interdipendenza dei vari componenti. D’altra parte, queste espressioni sono delle rappresentazioni mentali che si costruiscono nel corso delle interazioni con altri membri della comunità dei parlanti. Fanno parte del lessico dell'italiano e quindi devono essere acquisite da chi impara la lingua italiana per poter comunicare in modo efficiente. Dato che comunicare significa trasmettere messaggi vincenti (Balboni, Caon 2015), l'efficacia della comunicazione è altamente condizionata dagli aspetti culturali e, nelle interazioni interculturali, la conoscenza degli idioms contribuirà a risolvere quella parte del messaggio che non viene sempre espressa esplicitamente. Inoltre, quando Johnson-Laird afferma che «Idioms are the poetry of daily discourse» (1993, ix), si può intendere che le unità lessicae li complesse che costituiscono l'universo del linguaggio figurato rispetto alle semplici parole, non siano l'appannaggio della retorica di poeti e scrittori né degli slogan pubblicitari, ma facciano assolutamente parte delle interazioni quotidiane tra membri della stessa comunità linguistica. Per questa ragione, la competenza lessicale non può prescindere dagli aspetti idiomatici.

Le prime riflessioni in ambito glottodidattico sulla natura, sullo sviluppo della competenza lessicale e sui fenomeni di lessicogrammatica $^{3}$ quali le collocazioni, le espressioni idiomatiche e i chunks lessicali compaiono negli anni Novanta con le ricerche di Lewis (1993, 1997). Levorato $(1993,104)$ definisce la «figurative competence» come l'abilità di comprensione e di produzione di metafore e di forme idiomatiche. Più in generale, si riferisce all'uso figurato e traslato del linguaggio supportato dallo sviluppo di una consapevolezza metalinguistica che consente al parlante di comprendere cosa l'interlocutore voglia trasmettere realmente, quale sia la vera intenzione comunicativa al di là di quanto viene letteralmente espresso attraverso il confronto con una forma idiomatica corrispondente nella madrelin-

3 Uno dei principi del Lexical Approach di Lewis recita che «la dicotomia grammati$\mathrm{ca} /$ lessico non è valida [...] la grammatica come struttura è subordinata al lessico [e] la lingua consiste di lessico grammaticalizzato e non di grammatica lessicalizzata» (Porcelli 2004, 41). 
gua dell'apprendente. Per la studiosa, questa competenza si acquisisce gradualmente attraverso una serie di abilità, ossia:

- conoscere il significato di una parola e le sue relazioni di carattere sintagmatico e paradigmatico;

- conoscere il valore denotativo e connotativo e i rapporti di significato tra le parole;

- saper sospendere la strategia referenziale;

- comprendere l'uso figurato di una parola e mettere in relazione il significato figurato e/o metaforico con il significato letterale;

- saper ricorrere alle informazioni testuali e contestuali per attribuire un significato coerente ad espressioni ambigue o non conosciute;

- saper produrre espressioni figurate.

Durante il processo di insegnamento/apprendimento della lingua straniera, l'acquisizione di tale competenza non è così palese. Ricordiamo che tra i problemi legati a un apprendimento fuori contesto (LS), si colloca nelle prime file la scarsa esposizione all'input linguistico-culturale nonché la limitatezza delle occasioni di interazione con i parlanti nativi. Questa situazione non è di certo favorevole a un contatto regolare e frequente con la lingua d'uso e quindi neanche con le espressioni idiomatiche più frequenti. In effetti, per l'apprendente di italiano in Camerun, il contatto con queste unità lessicali complesse avviene prevalentemente attraverso i testi presentati in classe o, dove le infrastrutture lo consentono, qualche film o canzone. Nonostante la vicinanza tipologica tra francese e italiano, l'apprendente francofono di italiano LS in Camerun incontra delle difficoltà nella decodifica e nella produzione delle espressioni idiomatiche dell'italiano.

\section{Contesto e strumenti di studio}

Il nostro corpus è stato raccolto durante tre semestri di corsi universitari di italiano LS presso studenti che hanno frequentato sia il laboratorio di lingua italiana il primo e il secondo anno sia il corso di traduzione del primo anno. ${ }^{4}$

Il campione di studio è composto da quattordici studenti del secondo anno universitario, iscritti a un corso professionale per futuri

4 Per approfondimenti sui contenuti del programma formativo dei futuri docenti di italiano per le scuole camerunesi alla Scuola Normale Superiore dell'Università di Maroua, si suggerisce il sito web dell'Unità d'italiano http://linguaitaliana-in-camerun.emonsite.com. 
insegnanti d'italiano nelle scuole secondarie del Camerun. ${ }^{5}$ Questi studenti hanno alle spalle cinque anni di studio dell'italiano a scuola per gli uni e per gli altri tre anni di studio universitario in un percorso di laurea triennale trilingue ${ }^{6}$ durante i quali hanno frequentato lezioni di lingua italiana a partire dal livello principianti. Al loro ingresso nella Scuola Normale Superiore presentavano un livello di competenza linguistico-comunicativa più o meno pari a un A2/B1 del Quadro Comune Europeo di Riferimento per le lingue. Secondo i testi che organizzano i programmi ufficiali di insegnamento delle lingue straniere nelle scuole secondarie, gli studenti provenienti dalle scuole dovrebbero raggiungere al termine del loro percorso un livello pari al $\mathrm{C} 1 .^{7}$

Il corpus preso in esame è costituito da una parte da 70 composizioni scritte su 5 temi diversi svolti nel corso di tre semestri universitari, dall'altra parte da frasi tratte da esercizi e attività di comprensione dei testi e di traduzione. Per raccogliere e analizzare le espressioni idiomatiche usate dai nostri studenti abbiamo preferito la composizione libera (da svolgere a volte in classe a volte come compito da fare wle sia frastica. Questo tipo di esercizio ci è sembrato adeguato anche per il fatto che nella produzione scritta libera gli studenti sono meno preoccupati dagli aspetti formali e tendono a focalizzare l'attenzione sul contenuto. Invece, per valutare la competenza nella comprensione e la traduzione delle espressioni idiomatiche, abbiamo proposto ai nostri studenti riflessioni su una settantina di espressioni idiomatiche raccolte nel corso delle nostre letture in classe $^{8} 0$, il più delle volte, liste di modi di dire raggruppati in categorie aventi in comune un tema o una parola, da tradurre e da inserire poi in un contesto. Durante queste attività le espressioni e le frasi idiomatiche venivano nella maggior parte dei casi presentate in modo decontestualizzato ${ }^{9}$ e poi inserite in frasi spontanee dopo o durante la loro spiegazione/traduzione.

5 L'italiano è inserito entro il sistema educativo camerunese dall'anno scolastico 2011-12 come lingua straniera a scelta, a parità di credito con lo spagnolo, il tedesco, l'arabo e il cinese.

6 L'università di Dschang offre dall'anno accademico 1995/1996 all'interno del Dipartimento di Lingue Straniere Applicate, un corso di Laurea Triennale con un'Unità di italiano.

7 A nostro parere, considerate le variabili ambientali e individuali che influenzano l'apprendimento dell'italiano nelle scuole secondarie in Camerun, quei testi ufficiali sono poco realistici nei tempi e negli obiettivi da raggiungere in cinque anni di studio. Cf. Kuitche Talé 2015; Mazoua Megni Tchio 2015.

8 I testi dialogici e i racconti brevi sono i principali tipi di testo che servono da corpus per le nostre lezioni.

9 Questa scelta è dovuta alla difficile reperibilità di testi contenenti forme idiomatiche diversificate. 


\section{$5 \quad$ Risultati}

\subsection{Problemi di comprensione e di traduzione delle espressioni idiomatiche}

Durante lo svolgimento degli esercizi di traduzione delle espressioni idiomatiche dall'italiano al francese abbiamo potuto analizzare 68 espressioni, le uniche che hanno visto la partecipazione di tutti i nostri quattordici studenti: 42 di queste espressioni erano presentate in campi tematici o lessicali (ad esempio espressioni fisse con la parola 'pane', con la parola 'luce', con i colori, con le parti del corpo umano, con il verbo 'essere', ecc.); le altre 26 espressioni sono state individuate in testi 0 attività occasionali. La maggior parte delle espressioni proposte presentano un minore grado di opacità e la trasparenza lessicale e semantica prende in considerazione il livello di competenza degli studenti e la vicinanza tipologica tra L1 e lingua target ossia il francese e l'italiano.

In questa sede abbiamo scelto di presentare gli esempi più significativi per illustrare i problemi di comprensione e di traduzione delle forme idiomatiche. Ė tuttavia necessario precisare che se alcune frasi idiomatiche dell'italiano trovano una corrispondenza fraseologica nella lingua francese, altre invece sono state descritte solo con il significato che l'espressione assume in francese, anche perché non tutte le espressioni idiomatiche, nel passaggio da una lingua a un'altra, mantengono lo stesso valore idiomatico. Del resto, come sostiene Porcelli, «lingue diverse non sono etichette diverse messe sugli stessi oggetti, ma modi diversi di classificare la realtà» $(2004,44)$.

\section{a. Dire pane al pane, vino al vino}

Traduzioni più frequenti degli studenti: ceil pour œil, dent pour dent aller droit au but

Espressione equivalente in francese: appeler chien chien et chat chat

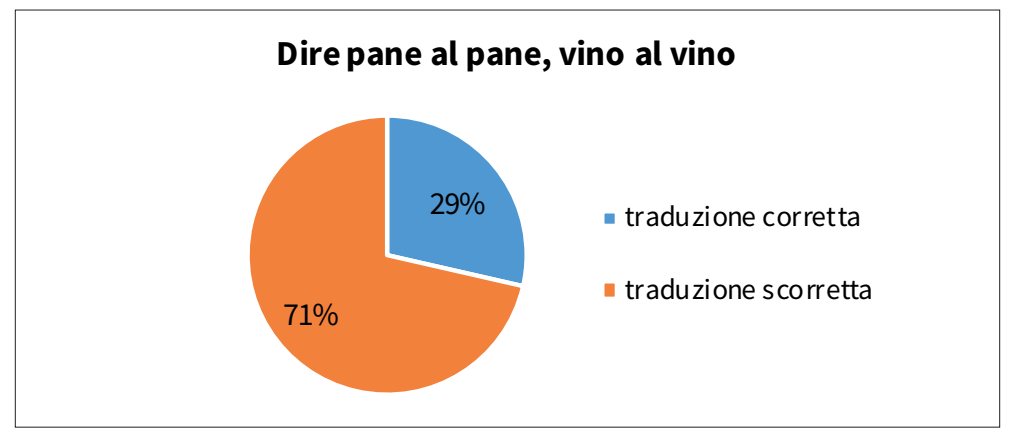


Per la traduzione di questa espressione, solo 4 studenti hanno risposto in modo corretto. La doppia presenza del termine 'pane' ha probabilmente indotto molti di loro a tradurre con un'espressione in lingua francese contenente un raddoppiamento del sostantivo.

\section{b. Vedere tutto nero}

Traduzioni più frequenti degli studenti: être fâché,

être dans la souffrance, voir du feu

Significato in francese: être pessimiste

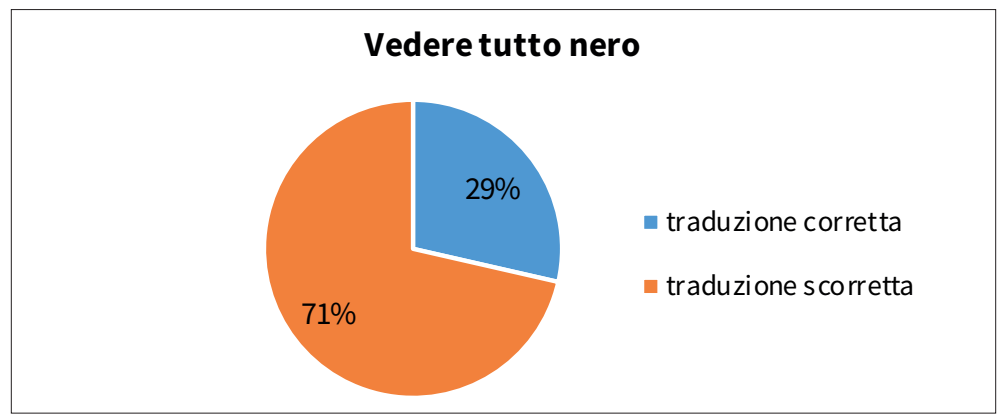

Anche per questa espressione solo 4 studenti hanno tradotto in modo corretto. Per influenza della cultura, tanti di loro hanno assimilato il 'nero' alla sofferenza.

\section{c. Darsela a gambe}

Espressione equivalente in francese: prendre ses jambes à son cou Traduzioni più frequenti degli studenti: se donner à fond (darci dentro), se remettre sur pied

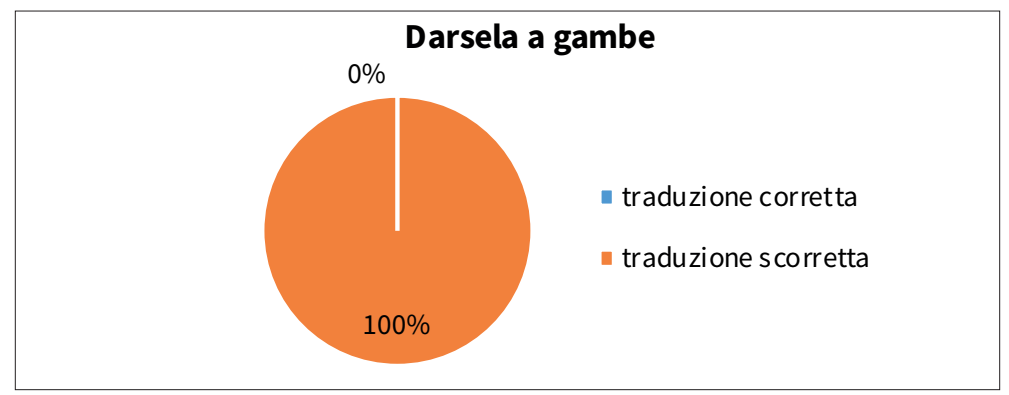


Per il suo grado di opacità, nessuno dei 14 studenti è stato in grado di decodificare questa espressione, nonostante esista un'espressione equivalente in francese. Questo è probabilmente dovuto anche al fatto che è un'espressione poco usata dagli studenti nelle loro interazioni quotidiane in $\mathrm{L} 1$.

\section{d. Alla luce del sole}

Espressione equivalente in francese: au vu et au su de tous

Traduzioni più frequenti degli studenti: à la lumière du soleil, du jour

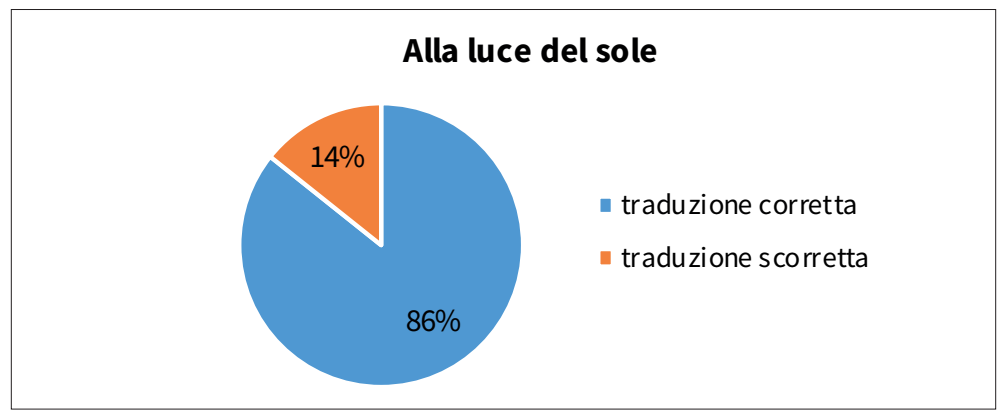

I 12 studenti che hanno interpretato l'espressione con il senso di 'à la lumière du jour' facendo così una traduzione letterale hanno saputo ugualmente inserirlo in un contesto, anche se non sono riusciti ad azzeccare l'espressione equivalente in francese.

\section{e. Fare orecchie da mercante}

Espressione equivalente in francese: faire la sourde oreille Traduzioni più frequenti degli studenti: avoir les oreilles de commerçant, parler toujours d'argent

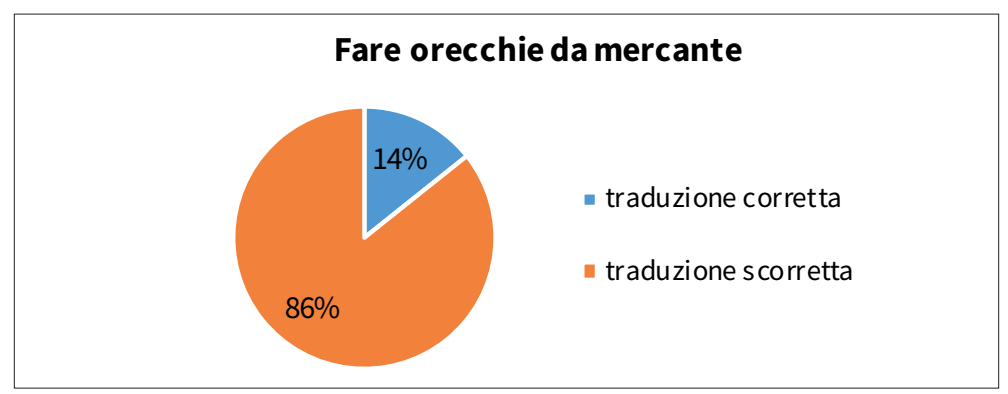


Solo 2 studenti sui 14 hanno decodificato l'espressione. Tutti gli altri hanno associato alle orecchie del mercante l'idea del commerciante a cui interessa solo guadagnare soldi.

\section{f. Fare complimenti}

Espressione equivalente in francese: faire des manières, se faire prier

Traduzioni più frequenti degli studenti: apprécier,

faire des compliments

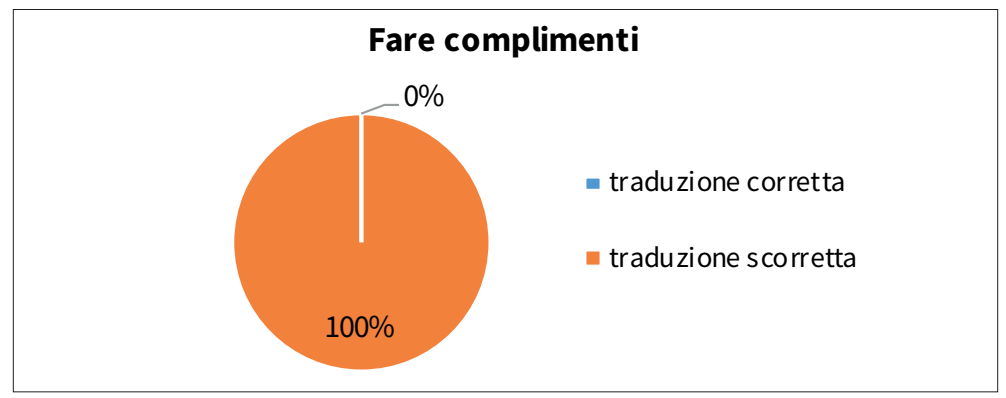

Tutti hanno dato un'interpretazione errata dell'espressione idiomatica. Anche se l'attività era esplicitamente centrata sulla comprensione dei modi di dire, quindi sugli usi figurati del linguaggio, nessuno l'ha percepita come tale e ne hanno fatto una traduzione letterale.

\section{g. Alzare i tacchi}

Espressione equivalente in francese: montrer les talons Traduzioni più frequenti degli studenti: se vanter,

se faire voir,

faire le mannequin

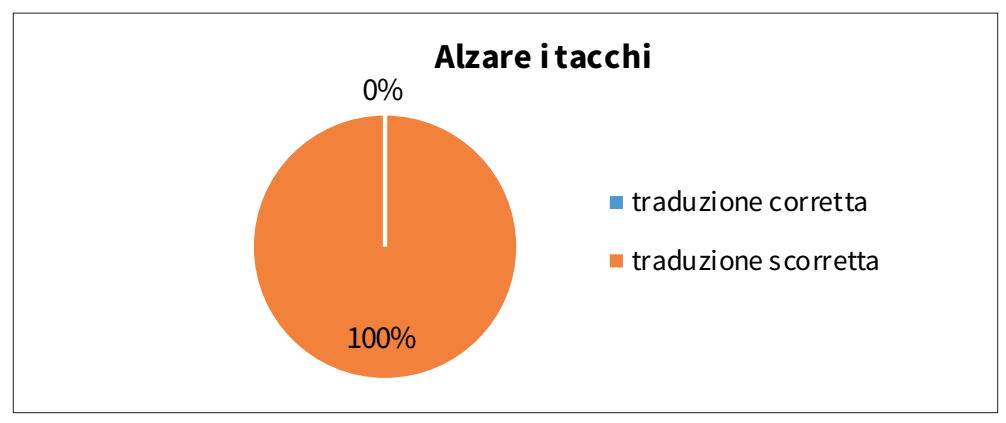


La totalità del nostro campione non è riuscita a tradurre questo idiotismo. Anche se, presi singolarmente gli elementi che lo costituiscono sembrano semplici e trasparenti, l'espressione in sé presenta un alto grado di opacità per cui nessuno è giunto al significato figurato.

La maggior parte delle frasi fisse ed espressioni idiomatiche capite e reimpiegate bene dagli studenti è abbastanza frequente nella comunicazione quotidiana in francese, sono ad esempio non avere una lira; mettere nero su bianco; passare una notte in bianco; dare alla luce; fare il doppio gioco; ecc. Gli studenti hanno probabilmente fatto riferimento ai livelli lessicali e semantici del francese, visto che i termini chiave delle espressioni sono da considerare nel loro senso denotativo.

Bisogna però rilevare che altre 3 espressioni idiomatiche sono risultate totalmente opache per i nostri studenti che non hanno fatto nessuna proposta di interpretazione nonostante le diverse sollecitazioni e strategie di facilitazione dell'insegnante. Si tratta delle seguenti espressioni: nuovo di zecca, tenere il broncio, mancare di una rotella.

\subsection{Problemi nella produzione delle espressioni idiomatiche}

Se la vicinanza strutturale e formale dell'italiano e del francese agevola l'acquisizione delle abilità ricettive, questa facilità non si verifica sempre quando si tratta di produrre un testo. In effetti, nelle produzioni scritte dei nostri studenti abbiamo potuto osservare la ricorrenza di enunciati che, seppur morfologicamente accettabili, avrebbero potuto avere una migliore incidenza semantica con l'impiego delle espressioni idiomatiche e di altre unità lessicali fisse. L'utilizzo di forme idiomatiche, oltre a dimostrare una buona competenza lessicale, rappresenta una risorsa linguistica importante. L'aspetto figurato, che crea uno scarto semantico, conferisce all'enunciato un valore stilistico che gli consente di sorprendere, di stupire o semplicemente di attrarre l'attenzione di chi legge o ascolta.

Abbiamo analizzato in questa ottica 70 composizioni scritte prodotte dal nostro gruppo di apprendenti. I testi prodotti hanno una lunghezza compresa tra 80 e 120 parole su temi vari. Le nostre osservazioni riguardano principalmente gli errori riconducibili a uno scorretto o mancato uso delle espressioni idiomatiche e altre forme fisse. Di seguito riportiamo le devianze dalla norma sintattica e pragmatica nell'uso di alcune locuzioni fisse. 
Vicky Mazoua Megni Tchio Apprendimento dell'italiano in Camerun

Tabella 1 Elenco di espressioni idiomatiche e altre locuzioni fisse usate in modo erroneo

\begin{tabular}{ll}
\hline Dati del corpus & Italiano corretto \\
\hline 'Amava tutto il mondo' & Voleva bene a tutti \\
\hline 'La mia amica viene di perdere suo padre' & La mia amica ha appena perso il padre \\
\hline 'Come d'abitudine' & Come al solito \\
\hline 'Lupare il mio aereo' & Perdere/mancare l'aereo \\
\hline 'Sono andata in ballata con gli amici' & Sono andata a fare una passeggiata \\
\hline 'Ho rimborsato i soldi' & Ho dato indietro/ ho restituito i soldi \\
\hline 'Torno a casa fino alle 17' & Non rincaso prima delle 17 \\
\hline 'Scopare la corte' & Passare la scopa/ pulire il cortile \\
\hline 'Siamo caduti d'accordo su delle & Ci siamo messi d'accordo su alcune \\
proposte' & proposte \\
\hline 'Non si intendono mai' & Non vanno mai d'accordo \\
\hline 'Rendere l'anima' & Morire \\
\hline 'Posare un lapino' & Fare un bidone \\
\hline 'Tagliare un bicchiere di vino' & Bere un bicchierino \\
\hline 'Ha fatto tutto per lui' & Si è fatto in quattro per lui \\
\hline 'Prendo una doccia' & Faccio la doccia \\
\hline 'Ha abbandonato' & Ha lasciato perdere/ Si è ritirato \\
\hline 'Faccio la mia cartella' & Preparo la mia cartella \\
\hline 'Vive ogni giorno come può' & Vive alla giornata \\
\hline 'Tirarsi dagli affari senza un graffio' & Cavarsela \\
\hline
\end{tabular}

Si nota che buona parte degli errori è riconducibile a due fenomeni principali:

- traduzione letterale/calco strutturale dal francese: in questi casi, non viene preso in considerazione il gruppo di parole interdipendenti che forma l'unità lessicale ma singole parole. Consapevoli delle somiglianze formali tra italiano e francese, gli studenti tendono a combinare le parole in italiano a partire da una semplice trasposizione del loro significato in francese. Questo calco strutturale si ha ad esempio in frasi quali 'amava tutto il mondo' per il francese 'il aimait tout le monde', 'tirarsi dagli affari' per 'se tirer d'affaire', 'venire di + infinito' per 'venir de + infinitif', 'rendere l'anima' per 'rendre l'âme', 'passare dal gallo all'asino' per 'passer du coq à l'âne', ecc. 
- influenza del francese popolare camerunese (FPC ${ }^{10}$ e del camfranglais: ${ }^{11}$ per quanto riguarda l'impiego di espressioni fisse, alcune deviazioni rilevate nelle composizioni scritte analizzate dimostrano una forte interferenza con il FPC e il camfranglais caratterizzati da una coloritura locale diversa dal francese normativo e standard. Si nota nelle espressioni così usate la presenza di molti calchi spesso conformi ad alcune strutture lessicali già stabilite dall'uso quotidiano di queste varianti. Presentiamo alcuni esempi:

Tabella 2 Errori da interferenza con il FPC e il camfranglais

\begin{tabular}{lll}
\hline Dati del corpus & $\begin{array}{l}\text { Espressione in camfranglais } \\
\text { e in FPC }\end{array}$ & Francese standard \\
\hline Ha fatto tutto per lui & Il a tout fait pour lui & Il lui doit tout/il l'a beaucoup aidé \\
\hline${ }^{*}$ Tagliare un bicchiere di vino & Couper une bière & Partager une bière \\
\hline${ }^{*}$ Vive ogni giorno come può & Il vit chaque jour comme il peut & Il vit au jour le jour \\
\hline${ }^{*}$ Torno a casa fino alle 17 & Je rentre à la maison jusqu'à 17h & $\begin{array}{l}\text { Je ne retourne pas à la maison } \\
\text { avant 17h }\end{array}$ \\
\hline
\end{tabular}

\section{Proposte didattiche per l'apprendimento delle espressioni idiomatiche in italiano LS}

Come già anticipato nel paragrafo 3 , la competenza linguistico-comunicativa, ovvero l'efficacia della comunicazione è altamente condizionata dalla conoscenza del linguaggio figurato. Si rivela quindi molto importante la profonda comprensione e il successivo reimpiego del senso figurato dei modi di dire e altre forme fisse nonostante la loro complessità.

L'analisi dei dati presentati rende conto del fatto che la comprensione, la traduzione e l'impiego delle espressioni idiomatiche da parte degli apprendenti di livello A2/B1 della Scuola Normale Superiore di Maroua si appoggia principalmente sulle loro conoscenze pregresse, sul significato letterale delle singole parole ma anche sull'esistenza o meno della stessa espressione nella loro L1, ossia il francese. Alcuni errori rilevati sono riconducibili alla somiglianza a livello formale dell'ita-

10 Con francese popolare camerunese (FPC) facciamo riferimento al francese parlato in Camerun, influenzato pesantemente dalle lingue e culture locali. Questa variante del francese è caratterizzata da alcune singolarità lessicali e morfosintattiche che possono essere all'origine di errori durante l'apprendimento dell'italiano (Kuitche Talé 2013).

11 Il camfranglais è un parlato misto che si è sviluppato dal contatto tra il francese, l'inglese e il pidgin English e che integra molti tratti delle parlate locali. È un parlato molto usato dai giovani in contesti informali. 
liano e del francese, altri alla trasposizione nella lingua di arrivo delle forme usate nelle varianti locali del francese che si discostano spesso dalla lingua standard. In effetti, si nota che gli apprendenti di lingua

tendono a trasferire le forme e i significati della lingua e cultura materna e la loro distribuzione nella lingua e cultura straniera. (Lado 1957, 2)

Si può dedurre proprio dalle loro produzioni e interpretazioni errate delle unità lessicali e sintattiche che, nonostante le due lingue siano tipologicamente affini, il transfer non è sempre positivo, anzi si può rivelare «il primo responsabile nella commissione di errori» (Spreafico 2006, 109).

La traduzione da una lingua a un'altra, per di più la traduzione delle espressioni fisse, richiama il concetto di traducibilità, relativo non solo agli aspetti linguistico-strutturali, ma anche agli usi e costumi, alle culture e civiltà dei popoli diversi. Nell'esercizio della traduzione è consueto notare la mancanza nell'altra lingua sia di un referente sia di una corrispondenza perfetta dell'enunciato proposto. Inoltre, trattandosi di unità lessicali complesse, la comprensione delle espressioni idiomatiche è un processo a diversi livelli. È quindi opportuno cercare di decrittare non le singole parole, bensì raggrupparle in base al senso globale dell'enunciato, proprio perché

la ricerca di senso deve superare i confini della parola isolata per dare conto della dimensione minima richiesta caso per caso. (Porcelli 2004, 50)

Cardona (2008) lamenta il fatto che nella riflessione teorico metodologica e nei testi per l'apprendimento dell'italiano si presti poca attenzione alle espressioni idiomatiche. Secondo il ricercatore, queste ultime vengono presentate in genere in un elenco decontestualizzato con la traduzione a fianco o confinato in brevi paragrafi alla fine delle varie unità didattiche in un confronto con una forma idiomatica corrispondente nella lingua madre dell'allievo. ${ }^{12}$ L'apprendimento delle forme idiomatiche sarebbe invece più efficace se venissero presentate in contesti significativi. Questo svilupperebbe negli allievi la competenza metalinguistica necessaria a far diventare tali espressioni patrimonio del lessico produttivo, oltre che ricettivo, giacché l'apprendimento delle forme idiomatiche rappresenta non solo un aspetto importante della competenza lessicale, ma assume anche una forte valenza pragmatica.

12 Come riferito prima nel paragrafo 3, anche per questa ricerca molte delle espressioni fisse presentate durante l'esperimento erano sia inserite in semplici frasi sia presentate sotto forma di lista avente in comune un tema o una parola. 
D’altro canto, in sede operativa, è sempre importante rispettare alcuni criteri di scelta delle espressioni idiomatiche da proporre in classe: la frequenza d'uso dell'espressione nella L2 e la difficoltà del vocabolario interno sono da considerare insieme al grado di trasparenza semantica e alla similarità dell'espressione nella L1 dell'apprendente. Inoltre, vista la loro pervasività nel lessico dell'italiano e considerati questi criteri di selezione, le attività per l'apprendimento delle espressioni idiomatiche andrebbero proposte sin dai livelli elementari.

Come riferito nei paragrafi precedenti, le espressioni idiomatiche sononella maggior parte dei casi delle unità lessicali costituite da parole che, prese singolarmente, non presentano ambiguità e possono essere comprensibili all'apprendente straniero. Quando invece vengono combinate con altre parole assumono spesso valori particolari. Una difficoltà nella comprensione delle espressioni così costituite potrebbe essere appunto come sapere quando parole semplici, che conosciamo bene, assumono un significato diverso se sono messe assieme. Consultare un dizionario sembra essere la prima evidenza, dato che, come ricorda Porcelli, «cercare sul dizionario anche le parole che si pensa di conoscere può riservare parecchie sorprese» (2004, 50). Sarà quindi compito del docente di lingua straniera addestrare gli apprendenti alla scelta e all'uso di dizionari validi.

Alcune tecniche (abbinamento, scelta multipla, completamento) ampiamente impiegate per l'apprendimento del lessico possono essere usate anche per l'apprendimento delle unità lessicali complesse.

Nello specifico, si potrebbe sperimentare in contesto di insegnamento dell'italiano LS, attività di lavoro sulle locuzioni fisse con la tecnica dell'abbinamento centrata sul significato e non sulla forma: in un'attività sui modi di dire con gli animali, si chiede agli studenti di abbinare le fotografie degli animali ai modi di dire (ad esempio, fotografia di un lupo con la bocca aperta e l'espressione 'in bocca al lupo'; fotografia di un rospo e l'espressione 'sputare il rospo' ecc.); si chiede poi agli studenti di collegare le stesse espressioni nella colonna A con le definizioni corrispondenti della colonna B. Un'attività successiva a questi esercizi di abbinamento potrebbe essere l'inserimento dell'espressione in un enunciato/contesto, o chiedere di trovare l'espressione corrispondente nella L1.

La scelta multipla è anche una tecnica valida per la comprensione delle espressioni idiomatiche: si chiede agli studenti di scegliere da una lista la parola corretta per completare l'espressione; si potrebbe diversificare questo esercizio con diversi campi lessicali. Ad esempio, si potrebbe lavorare su modi di dire con animali, con colori, con parti del corpo, con il cibo, con i verbi, ecc.

In un'altra attività, si presentano agli studenti delle fotografie che rappresentano i modi di dire in italiano con una parola specifica o un campo lessicale e si chiede loro di scrivere sotto ogni figura il 
modo di dire corrispondente, scegliendolo da una lista proposta. Si propone poi un esercizio di scelta binaria vero/falso sul significato dei modi di dire.

Le attività appena elencate servono a familiarizzare gli apprendenti con le espressioni idiomatiche, addestrandoli al loro riconoscimento e alla loro comprensione. Tuttavia, se aiutano a sviluppare una competenza ricettiva di queste strutture lessicali complesse, non sono così efficaci in fase di produzione, dato che questi esercizi, per la maggior parte, si basano su liste di espressioni decontestualizzate. Una migliore acquisizione delle espressioni idiomatiche che consenta un loro uso strategico e una conseguente rilevanza pragmatica in situazioni interattive, impone che siano inserite in un contesto. La loro raccolta potrebbe avvenire tanto in contesti didattici che extradidattici, ascoltando canzoni, guardando film o leggendo testi in lingua italiana, per una successiva analisi in classe.

Abbiamo riferito all'inizio di questa analisi che, in modalità LS, l'esposizione molto ridotta all'input linguistico in italiano e le scarse possibilità di interazione con i nativi costituiscono fattori limitanti per l'apprendimento delle espressioni idiomatiche. Per i teorici del Lexical Approach (Lewis 1993, 1997), molto lessico viene appreso in modo incidentale attraverso la lettura. Lo sviluppo dell'abilità di lettura rappresenta senza dubbio una componente fondamentale per lo sviluppo della competenza lessicale. La lettura è dunque uno strumento particolarmente adeguato per l'espansione lessicale. L'apprendente di italiano nel nostro contesto deve quindi necessariamente avvicinarsi alla lettura dei testi, principalmente dei testi autentici, per poter reperire, percepire e comprendere l'espressione idiomatica a partire da un contesto situazionale. In una prospettiva inferenziale, le attività di classe potranno vertere sulla rassegna e il riutilizzo di tali espressioni.

Essendo la conoscenza delle espressioni idiomatiche e dei riferimenti culturali ad esse sottese molto importante per il raggiungimento della competenza comunicativa, consigliamo a livello operativo in classe 0 anche a casa in autoapprendimento, oltre alle molteplici risorse online, alcuni manuali ${ }^{13}$ a nostro avviso dedicati nel loro assetto metodologico alla didattica delle espressioni idiomatiche. Possiamo segnalare Pichiassi, Zaganelli 2003 e Aprile 2008.

13 Ci limitiamo a questi due manuali perché sono immediatamente accessibili ai nostri studenti in quanto sono reperibili nella biblioteca del Dipartimento di Lingue Straniere della Scuola Normale Superiore dell'Università di Maroua. 


\section{Bibliografia}

Aprile, G. (2008). Italiano per modi di dire. Firenze: Alma.

Balboni, P.E.; Caon, F. (2015). La comunicazione interculturale. Venezia: Marsilio.

Cacciari, C.; Tabossi, P. (eds) (1993). Idioms. Processing, Structure and Interpretation. Hillsdale, $\mathrm{NJ}$ : Lawrence Erlbaum.

Cardona, M. (2008). «La comprensione e produzione di idioms. Aspetti psicolinguistici e riflessioni glottodidattiche». Studi di glottodidattica, 3, 45-64. http://ojs.cimedoc.uniba.it/index.php/glottodidattica/article/viewFile/200/71.

Casadei, F. (2007). Lessico e semantica. Roma: Carocci.

Covino Bisaccia, M.A. (1996). I discenti francofoni e l'apprendimento dell'italiano. Perugia: Guerra.

Glucksberg, S. (1993). «Idioms, Meanings and Allusional Content». Cacciari, Tabossi 1993, 3-26.

Johnson-Laird, P. (1993). «Introduction». Cacciari, Tabossi 1993, vii-x.

Kuitche Talé, G. (2013). «Variazione diatopica del francese e didattica dell'italiano L2: i transfer negativi degli apprendenti camerunensi nell'italiano». Italiano LinguaDue, 2, 79-95. http://riviste.unimi.it/index.php/ promoitals/article/view/3753.

Kuitche Talé, G.; Pallante, G. (a cura di) (2015). 1995-2015. 20 anni d'insegnamento dell'italiano L2 in Camerun. Bilancio e prospettive. Italiano LinguaDue, 2. https://doi.org/10.13130/2037-3597/6807.

Kuitche Talé, G. (2015). «Il peso delle variabili ambientali nella didattica dell'italiano L2 nelle scuole camerunensi». Kuitche Talé, Pallante 2015, 84-93.

Lado, R. (1957). Linguistics across Cultures. Applied Linguistics for Language Teachers. Ann Arbor: University of Michigan Press.

Levorato, M.C. (1993). «The Acquisition of Idioms and the Development of Figurative Competence». Cacciari, Tabossi 1993, 101-28.

Lewis, M. (1993). The Lexical Approach. Hove: Language Teaching Publication.

Lewis, M. (1997). Implementing the Lexical Approach. Hove: Language Teaching Publication.

Mazoua Megni Tchio, V. (2015). «Le variabili individuali e la percezione dell'italiano LS negli apprendenti delle scuole secondarie in Camerun». Kuitche Talé, Pallante 2015, 94-110.

Perrin, L. (2003). «Citations, lexicalisation et interprétation des expressions idiomatiques». Authier-Revuz, J.; Doury, M.; Reboul-Toure, S. (éds), Parlerdes mots: le fait autonymique en discours. Paris: Presses Sorbonne Nouvelle, 281-92.

Petrocelli, E. (2011). «Insegnare italiano L2 a adolescenti». Diadori, P. (a cura di), Insegnare italiano a stranieri. Milano: Le Monnier, 218-29.

Pichiassi, M.; Zaganelli, G. (2003). Contesti italiani. Viaggi nell'italiano contemporaneo attraverso i testi. Materiale per la didattica dell'italiano L2. Perugia: Guerra.

Porcelli, G. (2004). Comunicare in lingua straniera. Il lessico. Torino: UTET.

Spreafico, A. (2006). «Analisi contrastiva Italiano/Francese: il caso di discenti di italiano L2 Camerunesi». Itals, 12. Perugia: Guerra. 


\title{
EL.LE
}

Vol. 9 - Num. 1 - Marzo 2020

\section{The Use of the Verb Run in English Learner-Narratives An Analysis of Verb Constructions Influenced by Different L1s}

\author{
Martina Irsara \\ Libera Università di Bolzano, Italia \\ Ulrike Domahs \\ Institute for German Linguistics, Philipps-Universität Marburg, Deutschland
}

\begin{abstract}
The present paper investigates the use of the verb RUN in texts compiled in English by three groups of multilingual learners in the North of Italy. The participants spoke three different L1s: Ladin, Italian, and German. The main objective was to ascertain whether L1 imprints could be identified in the different groups, and whether the Ladin subjects behaved more similarly to their Italian or German counterparts. Despite considerable homogeneity found across all groups, the findings surprisingly suggested a stronger closeness between the texts of Ladin and German speakers, and not - as the typological relationship between Ladin and Italian would suggest - between Ladin and Italian speakers. The present data implicate that multilingual speakers bring to bear composite cognitive and typological systems when they construct texts in English as a third or further language, and text features are usually not easily attributable to a typologically clearly defined L1.
\end{abstract}

Keywords Learner-texts. English as a third or fourth language. Ladin frog story. Satellite-framed languages.

Summary 1 Introduction and Research Questions. - 2 Previous Investigations and Theories. -3 Hypotheses of the Current Study. - 4 Method. - 4.1 Participants and Writing Task. - 4.2 Procedure. -5 Results. - 5.1 Similarities between the Groups. - 5.2 Differences between the Groups. - 5.3 L1-Influence on English. - 6 Discussion and Conclusions.

\section{Peer review}

Submitted 2019-03-08

Accepted 2020-05-10

Published 2020-07-30

\section{Open access}

(c) 2020 | () Creative Commons Attribution 4.0 International Public License 


\section{Introduction and Research Questions}

It seems indisputable that multilingual language learners might be influenced in a dynamic way by more than one language in their acquisition process and language use (Cenoz, Hufeisen, Jessner 2001; Jessner 2006, 2008). However, the exact role of the background languages in further language (Ln) learning is controversial. According to the L1 factor hypothesis, the L1 is the privileged source of transfer at the initial state of L3 learning, while other hypotheses postulate that the L1 is favoured only when a particular property has not been acquired in the L2 yet, and that it is the L2 that takes on the strongest role in L3 learning (Bardel, Falk 2007; Falk, Bardel 2010; Hermas 2010, 2015). The typological primacy model suggests that L3 or Ln development is influenced by what the multilingual learner perceives to be the typologically closest language in his or her repertoire, whether or not such transfer is facilitative (Rothman 2011, 2015). The scalpel model of third language acquisition argues that transfer happens property by property from any previously acquired language and that it is influenced by "additional factors pertaining to the relevant properties, such as processing complexity, misleading input, and construction frequency in the target L3" (Slabakova 2017, 655).

The present study aimed to identify the level of similarity in the use of verb-phrases containing RUN across three learner groups representing three L1s: (i) Dolomitic Ladin, (ii) German (the Austrio-Bavarian dialect spoken in South-Tyrol), and (iii) Italian. ${ }^{1}$ The research participants learnt English in a region where English is taught mainly as an L3 and L4, after Ladin, Italian, and German. The question was raised whether the Ladin subjects, who were proficient in both Italian and German, were closer to the Italian or to the German speakers in their use of the verb RUN. The possible influence of Ladin, Italian, and German on the target language - English - was investigated, and a possible rationale for a number of similarities and anomalies between the three L1 groups was proposed. The findings of this study are explained from the perspective of cross-linguistic influence. ${ }^{2} \mathrm{An}$ swers to the following questions were sought:

1. What are the similarities between the Ladin, Italian, and German learners of English in the use of the verb RUN?

Ulrike Domahs carried out the statistical analysis and is responsible for sections 4.2 and 5.2. Martina Irsara initiated the study and is responsible for all other sections.

1 The form RUN in italicised small capitals is used to indicate different tense and person conjugations of the verb.

2 The terms 'cross-linguistic influence' and 'transfer' are used interchangeably in the current study. 
2. Which L1 group differentiates itself most? Do the Ladin speakers behave more similarly to the Italian or to the German speakers in the use of the verb RUN in their narratives?

3. Do the L1s Ladin, Italian, and German have an influence on the target language English?

\section{Previous Investigations and Theories}

The high-frequency verb RUN has been classified as a manner-of-motion verb, since its stem includes information about how the figure physically moves (i.e. move fast on foot). Unlike manner-of-motion verbs, path verbs (e.g. exit, enter, get) and their adnominal and adverbial encodings (e.g. into, away) describe the trajectory the figure follows. Talmy $(1985,1991,2000,2016)$ considers languages to be verb-framed (V) when they habitually express paths of motion in the main verb and manner optionally outside it with prepositional phrases, adverbs, or gerunds (e.g. Italian Entrò di corsa / correndo, 'He entered running'). Languages are called satellite-framed (S) when they characteristically encode manner-of-motion information in the verb root and paths in directional particles (e.g. English Peter ran out or German Peter rannte heraus). Although most Romance languages are seen as V-framed, Ladin displays S-framed characteristics. It possesses a restricted manner verb lexicon but a wide repertoire of path particles, which is arguably due to its mainly oral nature, as suggested by Irsara (2015).

The verb RUN can occur as a bare verb, providing no further elaboration of path beyond its inherent directionality, e.g. He started running (Slobin 1996a). V-framed languages have been shown to use bare motion verbs more often than S-framed languages (Berthele 2006; Slobin 1996a). Moreover, RUN can appear in a minus-ground or in a plus-ground clause:

"Minus-ground clauses" consist of bare verbs or verbs with satellites indicating direction of movement; "plus-ground clauses" have, in addition, one or more prepositional phrases encoding source and / or goal. (Slobin 1996a, 201)

In Slobin's (1996a) data, both the English (S-framed) and Spanish (Vframed) speakers use more plus-ground than minus-ground clauses. Moreover, Slobin (1996a) shows that English narratives tend to use more ground adjuncts than Spanish narratives. Similarly, Berthele (2006) shows that the number of plus-ground clauses exceeds the number of minus-ground clauses. However, his figures do not permit to draw conclusions about path elaboration with ground elements in $\mathrm{S}$-framed as opposed to V-framed languages. In line with the above, Spreafico (2009) shows that all the languages considered by him fa- 
vour the adnominal locus, represented mainly by prepositional phrases, to express paths of motion.

Complements of source and goal are often found with RUN. Endpoints are typically marked by the prepositions to, into, and onto, while sources are normally expressed by prepositional phrases headed by away, from, out, and off. The source marker away can occur alone (He ran away) or with a from phrase as complement (He ran away from him) (Huddleston, Pullum 2002). It is maintained by Von Stutterheim, Carroll, and Klein (2009) that German speakers refer to endpoints more often than speakers of other languages, e.g. English and Dutch. In general, goals are expressed more often than sources. Evidence has been found for the goal-over-source principle by Verkerk (2017) in 17 Indo-European languages.

Leaving a source or reaching a goal can involve crossing an enclosure or boundary. In S-framed languages (e.g. English and German), manner verbs like RUN can be used in boundary-crossing situations (He ran into the house / Er rannte ins Haus), whereas they are normally not employed to indicate the crossing of enclosures in V-framed languages (e.g. Italian), where path verbs seem to be preferred (Entrò in casa [correndo], 'He entered the house [running]') (Alonso-Alonso 2015; Filipović 2007; Slobin, Hoiting 1994).

The verb RUN can be preceded by the inceptive verbs START and BEGIN. Ladin and Italian have developed a rich repertoire of aspectual verbal periphrases, among which a number of periphrases for START / BEGIN, e.g. Ladin mëte man a, de / scomencè $a$, de / se mëte a or Italian (in)cominciare a / mettersi a / prendere a / venire a. Gallmann, Siller-Runggaldier, and Sitta (2007) note that German normally prefers adverbial expressions (Plötzlich regnete es, 'suddenly it rained') over periphrastic START / BEGIN expressions (e.g. Es fing an / begann $z$ u regnen 'It started / began to rain'). Although adverbial phrases can replace START and BEGIN in Ladin and Italian as well, this seems to be the case in German in particular.

\section{Hypotheses of the Current Study}

A high degree of similarity between the groups was expected in the present study, due to the subjects' analogous multilingual biographies. Because of the nature of the stimulus material used for the analysis, it was expected that all the groups would use RUN mainly in the sense of 'fast motion' and 'escape' (Glynn 2014; Gries 2006). It was foreseen that the sense of 'escape' would be expressed frequently with the phrasal verb RUN away and that RUN would therefore be found more often with sources than with goals.

The speakers of German (S-framed) were expected to use more locative adverbs and prepositions with the verb RUN than the speak- 
ers of Italian (V-framed). Similarly, the English texts by the Ladin speakers were expected to exhibit a large number of locative particles, due to the large use of these in Ladin. Vice versa, the narratives by the Italian subjects were expected to have a higher percentage of RUN as a bare verb, in line with Slobin's (1996a) and Berthele's (2006) findings.

Germans were expected to express targets more often than Italians, in line with findings by Von Stutterheim, Carroll, and Klein (2009). In the expression of the meaning of 'escape', the German speakers were expected to use the phrasal verb RUN away more often than the Italian and Ladin speakers. While German has forms that are similar to the English one, WEGLAUFEN, WEGRENNEN, 'away + run', corresponding verbs in Italian are normally FUGGIRE, SCAPPARE, 'escape' rather than the analytical form CORRERE VIA, 'run + away'. Similarly, the Ladin equivalent is a reflexive form of SCIAMPEे, 'escape', and not SALTE DEMEZ, 'run + away'. In this respect, the Ladin participants were therefore expected to behave like the Italian speakers.

If manner verbs (such as CORRERE, 'run') are normally not used in Italian to describe the crossing of enclosures, the Italian participants were supposed to use the manner verb RUN less often than the German subjects when the path involved crossing a boundary. Also due to an L1 influence, the Italian and Ladin speakers were assumed to have a stronger tendency than the German speakers to use START and $B E G I N$ in their English texts.

\section{Method}

\subsection{Participants and Writing Task}

The data were provided by 94 learners of English, who formed three groups according to their L1s: Ladin (41 participants); Italian (21 participants); German (32 participants). All participants were multilingual and could speak Italian, German, and English. The Italian and German subjects learnt English as an L2 and L3, whereas the Ladin speakers learnt it as an L4 after Ladin, Italian, and German.

The participants were estimated to be at a B1-B2 level of English according to the Common European Framework of Reference. At the moment of data collection, the study participants were attending the final year of upper secondary school, or they had just started their English courses at university.

The data were collected through a shortened version of Mayer's (1969) picture-story Frog, where are you?, which depicts the adven-

3 See Iacobini, Masini $(2007,2009)$ for the aspectual meaning of correre via, 'run away'. 
tures of a boy who undertakes a search for his lost pet frog. The writing task was carried out on the basis of 18 black-and-white pictures that had been selected out of 24 . During the task, which was anonymous and unrehearsed, collaboration and support materials were not permitted.

\subsection{Procedure}

After the digitization of the texts, the sentences that contained instances of RUN were isolated for ease of analysis. Answers to the questions in Section 1 were sought by observing: (i) the frequency of occurrence and distribution of the verb RUN; (ii) the locative prepositions and adverbs accompanying RUN; (iii) the occurrence of the inceptive verbs START and BEGIN; (iv) the nominal locative specification in the RUN-events.

Quantitative and statistical analyses were carried out on the basis of the raw data, and qualitative reflections were made in order to broaden understanding of the data and answer why-questions.

To test whether Ladin children produced constructions in written language production that were more similar to the constructions used by learners with German L1 than to those occurring in participants with Italian L1, we fitted generalized mixed effects logistic regression models (e.g. Baayen 2008; Baayen, Davidson, Bates 2008).

For the mixed effects analysis we used $\mathrm{R}$ and the 'lme4' package (Bates et al. 2015). We first fitted generalized mixed effects models for the overall occurrence of the verb RUN, before we calculated models for the occurrence of different constructions with RUN, like started running, as dependent variables. In each model, we included the predictor Language group with the levels Ladin, German, and Italian as fixed effect, where we defined the level Ladin as baseline.

In the following, we report $\mathrm{z}$ - and $\mathrm{p}$-values for the mixed effects models if factors contribute significantly or marginally significantly to the models. In order to keep participant variation under statistical control, the factor participant was included as random effect.

\section{$5 \quad$ Results}

\subsection{Similarities between the Groups}

In all the groups, RUN was used around twice per learner and corresponded to approximately $4-5 \%$ of the total number of verb-phrases. ${ }^{4}$ The verb was used most often to describe the events depicted in the

4 A grand total of 206 verb-phrases headed by RUN occurred in the learner-narratives analysed. 

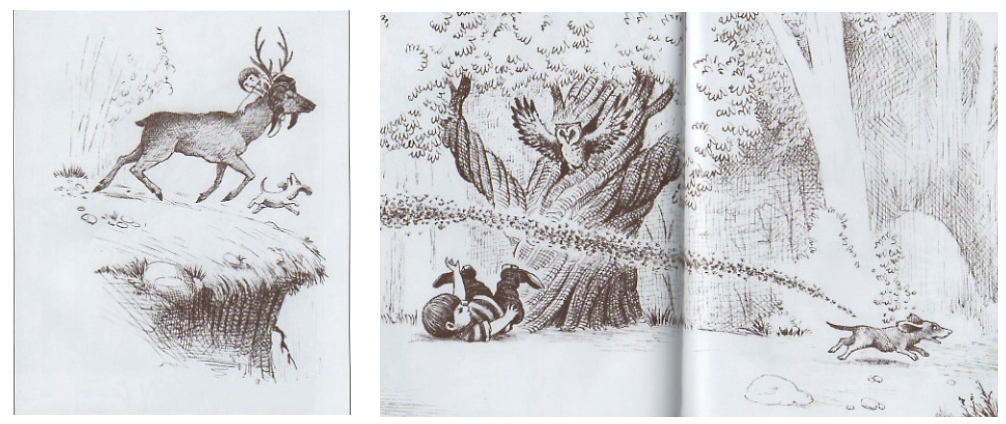

Figures 1-2 Deer approaching the riverbank and dog escaping from the bees (Mayer 1969)

images given below as figures 1 and 2, showing a deer and a dog fast approaching a high riverbank and the protagonist's dog escaping from a swarm of bees.

Table 1 shows that, in all the groups, more than $40 \%$ of the occurrences of RUN referred to figure 1. Figure 2 came next in terms of RUN-occurrences.

Table 1 Images with the highest number of occurrences of $R U N(100 \%=$ the total number of occurrences of the verb RUN)

\begin{tabular}{lrrr}
\hline Images & Ladin L1 & Italian L1 & German L1 \\
\hline Deer approaching the riverbank (fig. 1) & $43 \%$ & $49 \%$ & $51 \%$ \\
\hline Dog escaping from the bees (fig. 2) & $26 \%$ & $16 \%$ & $29 \%$ \\
\hline Total & $\mathbf{6 9 \%}$ & $\mathbf{6 5 \%}$ & $\mathbf{8 0 \%}$ \\
\hline
\end{tabular}

The verb RUN was most often followed by locative prepositions and adverbs, whereas it was a bare verb in less than half of its occurrences. The locative particle that accompanied the verb RUN most often was away. Table 2 illustrates that RUN was followed by away in more than $40 \%$ of its occurrences in the German and Ladin groups, whereas the percentage was lower in the Italian group. However, there was no statistically significant difference between the groups in the use of away with the verb RUN, although we saw differences when we compared the use of bare vs. non-bare verbs in general (see § 5.2). 
Martina Irsara, Ulrike Domahs The Use of the Verb Run in English Learner-Narratives

Table 2 RUN $+\operatorname{away}(x x)(100 \%=$ the total number of occurrences of the verb-phrase $R U N)$

\begin{tabular}{ll}
\hline L1 & Percentage of RUN + away $(\boldsymbol{x x})$ \\
\hline Ladin & $41 \%$ \\
\hline Italian & $29 \%$ \\
\hline German & $44 \%$ \\
\hline
\end{tabular}

In the majority of cases, RUN away was not post-modified by further elements, as in (1), where RUN away does not need an overt source adjunct, as the entity the subject is fleeing from is mentioned previously, namely an owl, referred to by the superordinate expression $a$ bird of prey.

(1) The boy hid from the bird of prey and ran away. (Ladin L1)

RUN away was accompanied by a prepositional or adverbial element in less than half of its occurrences, as table 3 shows. The percentage was the highest in the Italian group. Hence, when the Italian subjects used the phrasal verb RUN away, they often added further information. However, the difference between the groups did not reach statistical significance in the use of RUN away with further elements.

Table 3 RUN + away $+x x(100 \%=$ the total number of occurrences of RUN away)

\begin{tabular}{ll}
\hline L1 & Percentage of RUN + away $+\boldsymbol{x} \boldsymbol{x}$ \\
\hline Ladin & $23 \%$ \\
\hline Italian & $40 \%$ \\
\hline German & $30 \%$ \\
\hline
\end{tabular}

The verb-phrase RUN was most often followed by directive particles, but these were not always accompanied by noun-phrases specifying the $R U N$-trajectory further.

In (2), RUN is followed by the preposition of direction to and a noun-phrase indicating the deer's destination. The sentences in (3) and (4) exemplify the use of the verb RUN without any nominal locative specification.

(2) The deer ran to a river with the boy on its head. (Ladin L1)

(3) This animal began to run very fast. (Ladin L1)

(4) The dog ran away, so fast he was able to run. (Ladin L1)

Noun-phrases accompanied RUN in one quarter of the total number of occurrences of the verb, whereas in $75-76 \%$ of its occurrences, RUN was not accompanied by any nominal or pronominal locative specification. Table 4 shows similar percentages for each group, with a dif- 
ference which is far from being statistically significant. The minusground clauses therefore exceeded the plus-ground clauses.

Table 4 Percentages of RUN with and without locative particles and further nominal / pronominal locative specification $(100 \%=$ the total number of occurrences of the verb-phrase $R U N)$

\begin{tabular}{lrr}
\hline L1 & $\begin{array}{r}\text { Percentage of RUN + locative } \\
\text { particles + nominal / pronominal } \\
\text { locative specification }\end{array}$ & $\begin{array}{r}\text { Percentage of RUN (+ locative } \\
\text { particles) without nominal / } \\
\text { pronominal locative specification }\end{array}$ \\
\hline Ladin & $25 \%$ & $75 \%$ \\
\hline Italian & $24 \%$ & $76 \%$ \\
\hline German & $25 \%$ & $75 \%$ \\
\hline
\end{tabular}

The verb RUN was found more often with sources than with targets [tab.5]. In all the groups, RUN was used to describe a subject leaving a source location in more than $40 \%$ of its occurrences. Explicit and implicit references to sources were found especially in the Ladin and German groups, where the percentages were similar. However, neither group distinguished itself from the others significantly. As with the sources, the verb RUN was followed by targets especially among the German and Ladin speakers, whereas this was not often the case in the Italian group, as illustrated in the third column of table 5. However, the rather low figures in this column did not reach statistical significance.

Table 5 RUN + (implicit and explicit) source and $R U N+$ target $/$ direction $(100 \%=$ the total number of occurrences of the verb-phrase $R U N$ )

\begin{tabular}{lrr}
\hline L1 & Percentage of RUN + source & $\begin{array}{r}\text { Percentage of RUN + target / } \\
\text { direction }\end{array}$ \\
\hline Ladin & $48 \%$ & $17 \%$ \\
\hline Italian & $41 \%$ & $10 \%$ \\
\hline German & $47 \%$ & $20 \%$ \\
\hline
\end{tabular}

The source was marked mainly by away (from) and out of. While in (5) the dog has escaped from the bees, in (6) the boy has just left his house.

(5) The dog was afraid and ran away from the bees. (Ladin L1)

(6) He ran out of the house to help the dog. (German L1)

A frequent use of the lexical aspectual verbs START and BEGIN came to light in the analysis of the learner data. In the texts written by the Ladin and Italian speakers, RUN was preceded by START and BEGIN in one quarter of its occurrences. The verb RUN was accompanied by START 
and BEGIN less often in the German group [tab. 6]. However, the difference between the groups turned out to be statistically non-significant.

Table 6 Start $/$ begin running $/$ to run $(100 \%=$ the total number of occurrences of the verb-phrase RUN)

\begin{tabular}{ll}
\hline L1 & Percentage of start / begin running/to run \\
\hline Ladin & $25 \%$ \\
\hline Italian & $25 \%$ \\
\hline German & $18 \%$ \\
\hline
\end{tabular}

\subsection{Differences between the Groups}

The Ladin group was closer to the German than to the Italian group in the distribution of the verb RUN through the narratives. In the story sections accompanying 6 images out of 18, a similar concentration of $R U N$ verb-phrases was found in the Ladin and German groups, whereas the Ladin group was closer to the Italian group only in 3 images.

Table 1 above showed that the concentration of the verb RUN in figures 1 and 2 was strongest among the German speakers, who differentiated themselves from the Ladin speakers most (80\% in the German group vs. 69\% and 65\% in the Ladin and Italian groups). However, a consideration of figure 2 on its own made the Italian group stand out (16\% in the Italian group vs. $26 \%$ and $29 \%$ in the Ladin and German groups). Unlike the German and Ladin groups, the Italian speakers showed a reluctance to describe the dog's escape from the swarm of bees with a RUN verb-phrase.

The unwillingness among the Italian speakers to use a RUN verbphrase to describe an act of fleeing was confirmed in another story section. Ladin and German speakers used RUN verb-phrases in the escaping scene depicted in figure 3 below, which shows the story protagonist bracing himself against an owl with wings spread wide apart above him, whereas none of the Italian speakers used a RUN expression in this story section.

Vice versa, a stronger use of RUN verb-phrases among the Italian than among the Ladin and German speakers was ascertained in the story section referring to figure 4 below, which shows the story protagonist standing outside his bedroom window with his pet dog in his arms. The dog has just fallen from the window, smashing a jar where its head was stuck. In the Italian group, 10\% of the RUN occurrences were used in this story section, as exemplified in (7), whereas in the Ladin and German groups only $4 \%$ and $5 \%$ of the RUN instances were found in this scene.

(7) The boy was very worried and immediately he ran out to help his friend, the dog. (Italian L1) 


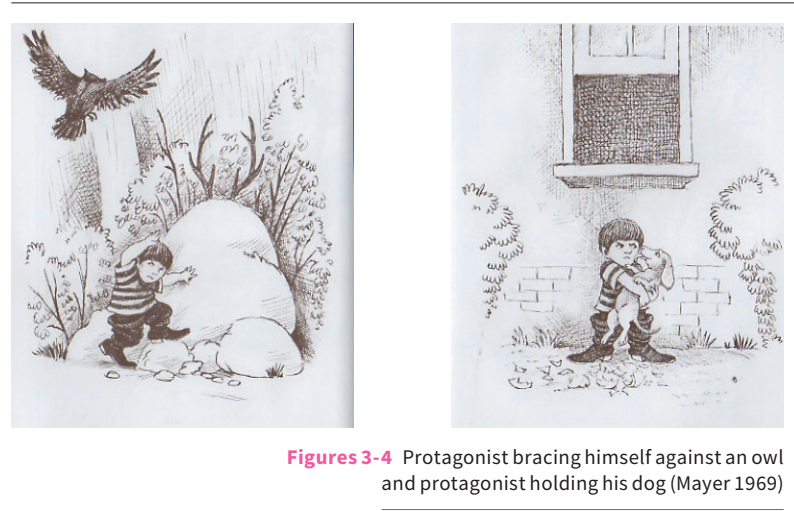

Despite the preference for non-bare verbs in all the groups, the Ladin and German subjects used locative prepositions and adverbs with the verb RUN more often than their Italian counterparts [tab. 7]. Vice versa, the Italian speakers had a higher percentage of RUN as a bare verb. For the occurrence of bare verbs vs. non-bare verbs, there was a trend for the Italian group to use RUN as a bare verb more often than the Ladin group [tab. 8].

Table 7 Percentages of RUN followed by locative particles and percentages of RUN occurring as a bare verb $(100 \%=$ the total number of occurrences of the verb-phrase $R U N)$

\begin{tabular}{lrr}
\hline L1 & $\begin{array}{r}\text { Percentage of RUN + locative } \\
\text { particle }\end{array}$ & Percentage of RUN as a bare verb \\
\hline Ladin & $66 \%$ & $34 \%$ \\
\hline Italian & $51 \%$ & $49 \%$ \\
\hline German & $66 \%$ & $34 \%$ \\
\hline
\end{tabular}

Table 8 Logistic regression model for the occurrences of the verb types bare vs. non-bare

\begin{tabular}{lrrrr}
\hline & Estimate & Std.Error & z.value & p.z \\
\hline (Intercept) & 0.68504107 & 0.2352883 & 2.91149684 & 0.0035 \\
\hline German group & -0.01243297 & 0.3637368 & -0.03418124 & 0.9727 \\
\hline Italian group & -0.64090845 & 0.3763734 & -1.70285248 & 0.0885 \\
\hline
\end{tabular}

The Italian group also differentiated itself in the analysis of boundary-crossing situations, as in (6) above, where the protagonist had to cross the doorway to go outside. The Italian group had the highest percentage of $R U N$-instances in boundary-crossing situations [tab. 9]. For the occurrence of RUN in boundary-crossing situations, the difference between the Ladin and Italian groups turned out to be significant [tab. 10]. 
Table 9 Boundary-crossing in the English learner-texts $(100 \%=$ the total number of occurrences of the verb-phrase RUN)

\begin{tabular}{ll}
\hline L1 & Percentage of $\boldsymbol{R}$ UN in boundary-crossing situations \\
\hline Ladin & $4 \%$ \\
\hline Italian & $20 \%$ \\
\hline German & $8 \%$ \\
\hline
\end{tabular}

Table 10 Logistic regression model for the occurrence of RUN in boundary-crossing situations

\begin{tabular}{lrrrr}
\hline & Estimate & Std.Error & z.value & p.z \\
\hline (Intercept) & 3.1135153 & 0.5109891 & 6.093114 & 0.000 \\
\hline German group & -0.6976015 & 0.6920736 & -1.007987 & 0.313 \\
\hline Italian group & -1.7025283 & 0.6208866 & -2.742092 & 0.006 \\
\hline
\end{tabular}

\subsection{L1-Influence on English}

The slightly larger use of directional particles by the German and Ladin speakers (66\%) than by their Italian counterparts (51\%) might be an L1 effect. The figures in table 7 seem to confirm the hypothesis that speakers of S-framed L1s (German) have a stronger tendency to employ locative particles than speakers of V-framed languages (Italian), who appear to favour bare motion verbs (Berthele 2006; Slobin 1996a). The statistically marginal difference between the Ladin and Italian groups of learners in their use of directional particles might find an explanation in the wide use of locative particles in the Ladin language.

Crosslinguistic influence from the learners' L1 was detected in the extent of the participants' use of RUN away. Although the group differences in the use of RUN away could not be statistically confirmed [tab. 2], the lower percentage in the Italian (29\%) than in the Ladin (41\%) and German (44\%) groups might nonetheless be interpreted as a possible L1 effect. Unlike the German and Ladin learners, the Italian speakers were unwilling to use RUN in the description of the dog escaping from the bees [fig. 2] and never used it to describe the boy hiding from a preying bird [fig. 3]. The one-word forms FUGGIRE and SCAP$P A R E$, 'escape' in Italian, might have restricted the use of the two-word phrase RUN away in the narratives written by the Italian speakers. Vice versa, the German prefixed verbs WEGLAUFEN and WEGRENNEN, 'away + run', might have prompted German participants to choose the similar phrase RUN away in their English texts. Since Ladin normally uses the form SCIAMPE to describe an act of fleeing, like Italian, the frequent use of RUN away in the Ladin group might be due to a German influence, or to the strong tendency to use syntagmatic verbs and locative adverbs in Ladin in general. 
Possible influence from their source language was assumed in the German learners' use of the verb RUN with targets. Low figures resulted and did not reach statistical significance [tab.5], but the hypothesis based on findings by Von Stutterheim, Carroll, and Klein (2009) that German speakers tend to indicate the target or goal of motion more often than speakers of other languages could be supported to some extent.

However, the minus-ground clauses exceeded the plus-ground clauses in each group [tab.4]. The results obtained by Berthele (2006), Slobin (1996a), and Spreafico (2009) could thus not be supported in the analysis presented here. It could not be confirmed that speakers of S-framed languages tend to add more ground adjuncts, as the separation of the German group from the Italian and Ladin groups was only $1 \%$.

Counter to expectations, the highest percentage of $R U N$-instances in boundary-crossing situations was found in the Italian group, with a statistically significant difference between the Italian and Ladin groups [tabs 9-10]. If it is true that verb-framed languages (including Italian) prefer main verbs of path (e.g. uscire, 'exit') rather than of manner (e.g. RUN) to indicate the crossing of enclosures, the frequent use of RUN in boundary-crossing situations in the Italian group is not explainable as an L1 effect.

As was foreseen, the Ladin and Italian research participants used START and BEGIN more often than the German-speaking subjects, which might be due to the stronger use of aspectual verbal periphrases in Ladin and Italian than in German and, therefore, explainable as an L1 effect. However, this could not be proved statistically, since the difference between the groups turned out to be non-significant [tab. 6].

\section{Discussion and Conclusions}

The comparative evaluation revealed considerable resemblance between the Ladin, Italian, and German groups of learners. The verb RUN was used to a similar extent and had an analogous distribution in the learner-narratives. Locative prepositions and adverbs most often followed RUN, which occurred as a bare verb in less than half of its occurrences. The directive particle away was the most common satellite that accompanied RUN, which most frequently occurred in a minus-ground clause, without further nominal locative specification. A preference for sources over goals was observed in all the groups, but neither sources nor targets were made explicit very often.

The study participants spoke different L1s, but they all learnt English in a multilingual context, so that the high degree of similarity between the groups might not be surprising. It was expected that 
learners at an intermediate level of proficiency would use the verb RUN widely to narrate a story based on a succession of events with numerous changes of place. The verb RUN is a basic verb that normally appears at pre-A1 Starters level and seems to be employed extensively at higher levels as well. Learners of English have been shown to overuse elementary verbs of motion and to ignore more specific manner-of-motion verbs that have a lower frequency of occurrence, and suggestions have been made to pay more explicit attention to motion-event descriptions in classrooms (Irsara 2017).

Although the research groups used RUN more often with sources than with targets, the findings cannot deny the sound goal-oversource principle revealed by Verkerk (2017). They rather confirm that language patterns are highly context-dependent, since chasing and escaping scenes that depict the protagonists fleeing from hazardous animals are central in Mayer's (1969) Frog, where are you? The findings also show that learners often leave information unexpressed when they narrate stories based on pictures, where a number of details are left to be inferred, e.g. sources and endpoints of motion events.

The research group that differentiated itself most was the Italian group, while the Ladin group of learners generally tended to be closer to the German speakers. The Italian participants showed a higher percentage of RUN as a bare verb (without locatives), confirming that speakers of V-framed languages (i.e. most Latin-based languages, e.g. standard Italian) have a stronger preference for bare verbs than speakers of S-framed languages (e.g. German) (Berthele 2006; Slobin 1996a). As expected, the Italian group of learners used the verb RUN less often than the other groups in escaping scenes. Despite the lack of statistical confirmation in this respect, the Italian speakers' reluctance to employ a RUN verb-phrase to indicate an act of fleeing was interpreted as a possible L1 effect, since it might be due to the formal dissimilarity between the English RUN AWAY and the Italian one-word form SCAPPARE. Against predictions, Italians used RUN more often than other speakers in the crossing of a boundary, where a statistically significant difference between the Ladin and Italian groups was found. However, the use of RUN in boundary-crossing situations was not always target-like in the Italian group. While running out of a house is possible and grammatically correct, the verb RUN is not appropriate to describe someone's exit from a window (e.g. run out of the window).

There might be a number of reasons why the Ladin speakers turned out to be closer to the German than to the Italian group of learners. Although Ladin belongs to the Romance language group, like Italian, it has also a number of characteristics in common with German. While certain Old Romance traits that are also present in current German varieties were lost in a number of later Romance 
languages, they survived in Ladin, probably due to linguistic contact with German-speaking communities (Benincà 1994). The widespread use of syntagmatic verbs in Ladin has for instance been suggested to be the result of different factors, i.e. the existence of similar constructions in vulgar Latin; language contact with German; and the alpine landscape, which seems to invite detailed descriptions of directions (Hack 2011). Referring to Rhaeto-Romance, Ascoli spoke of "materia romanza e spirito tedesco" (1880-83, 556). Ascoli's (188083) description of Ladin traits as Romance substance with a Germanic spirit points to the similarities that Ladin shares with both Italian and German. Moreover, Ladin speakers are proficient in Italian and German, so that they can be influenced by both languages in their production of English texts. However, German seems to be most often used for comparisons in English language teaching at school, so that this might encourage learners to draw upon German more than upon Italian. The Ladin speakers generally behaved more similarly to the German speakers, but they were closer to the Italian participants in the use of the inceptive verbs START and BEGIN, which they employed slightly more often in conjunction with RUN than the German speakers. Although statistically not confirmed, this was explained as a possible influence from the Ladin and Italian L1s, which appear to make wide use of verbal periphrases to express the beginning of events (Gallmann, Siller-Runggaldier, Sitta 2007).

Hence, few L1 effects could be identified in the multilingual groups of learners at an intermediate level of English proficiency. Although the L1 appeared to be somehow involved, it could not be argued to be the privileged source of transfer, in which case more inter-group differences would have been found. While it might be unproblematic to detect various forms of L1 influence in L2 learning, this becomes increasingly complex in L3 or Ln acquisition, particularly if the background languages are typologically related. In the acquisition of English as an L4 by speakers of Ladin, psychotypology might play a central role. Learners might perceive Ladin to be typologically distant from English because of its native status and regional nature, thus favouring German as the main source of influence. Given the high degree of similarity between the L3 and L4 groups of learners in this analysis, it might be ventured that L3 and L4 learners share more commonalities than sometimes assumed. 


\section{Bibliography}

Alonso-Alonso, R. (2015). "Boundary-Crossing Events in Spanish Learners of English. A Study on Motion into Events". Ibarretxe-Antuñano, I.; Hijazo-Gascón, A. (eds), New Horizons in the Study of Motion. Bringing together Applied and Theoretical Perspectives. Newcastle upon Tyne: Cambridge Scholars Publishing, 64-79.

Ascoli, G.I. (1880-83). "Annotazioni sistematiche al Barlaam e Giosafat soprasilvano: Saggio di morfologia lessicologica soprasilvana". Archivio Glottologico Italiano, 7, 406-602.

Baayen, R.H. (2008). Analyzing Linguistic Data. A Practical Introduction to Statistics. Cambridge: Cambridge University Press.

Baayen, R.H.; Davidson, D.J.; Bates, D.M. (2008). "Mixed-Effects Modeling with Crossed Random Effects for Subjects and Items". Journal of Memory and Language, 59(4), 390-412. https://doi.org/10.1016/j.jml.2007.12.005.

Bardel, C.; Falk, Y. (2007). "The Role of the Second Language in Third Language Acquisition. The Case of Germanic Syntax". Second Language Research, 23(4), 459-84. https://doi.org/10.1177/0267658307080557.

Bates, D. et al. (2015). Ime4: Linear mixed-effects models using Eigen and S4. $R$ package version 1.1-8. http://CRAN. R-project.org/package=lme4.

Benincà, P. (1994). La variazione sintattica: Studi di dialettologia romanza. Bologna: il Mulino.

Berman, R.A.; Slobin, D.I. (eds) (1994). Relating Events in Narrative. A Crosslinguistic Developmental Study. London; New York: Routledge.

Berthele, R. (2006). Ort und Weg. Die sprachliche Raumreferenz in Varietäten des Deutschen, Rätoromanischen und Französischen. Berlin; New York: Walter de Gruyter.

Berthele, R. (2013). "Disentangling Manner and Path. Evidence from Varieties of German and Romance". Goschler, Stefanowitsch 2013, 55-75.

Bylund, E.; Athanasopoulos, P. (2015). "Introduction. Cognition, Motion Events, and SLA". The Modern Language Journal, 99 (Supplement), 1-13. https:// doi.org/10.1111/j.1540-4781.2015.12175.x.

Cadierno, T.; Lund, K. (2004). "Cognitive Linguistics and Second Language Acquisition. Motion Events in a Typological Framework". Cadierno, T.; Lund, K. (eds), Form-Meaning Connections in Second Language Acquisition. Mahwah, $\mathrm{NJ}$; London: Lawrence Erlbaum Associates, 139-54.

Cadierno, T. (2008). "Learning to Talk about Motion in a Foreign Language". Robinson, P.; Ellis, N. (eds), Handbook of Cognitive Linguistics and Second Language Acquisition. New York: Taylor and Francis, 239-75.

Cadierno, T. (2017). "Thinking for Speaking about Motion in a Second Language. Looking back and forward". Ibarretxe-Antuñano 2017, 279-300. https:// doi.org/10.1075/hcp.59.12cad.

Cenoz, J.; Hufeisen, B.; Jessner, U. (eds) (2001). Cross-Linguistic Influence in Third Language Acquisition. Psycholinguistic Perspectives. Clevedon; Buffalo; Toronto; Sidney: Multilingual Matters.

Falk, Y.; Bardel, C. (2010). "The Study of the Background Languages in Third Language Acquisition. The State of the Art". International Review of Applied Linguistics in Language Teaching, 48(2-3), 185-219. https://doi. org/10.1515/iral.2010.009.

Filipović, L. (2007). Talking about Motion. A Crosslinguistic Investigation of Lexicalisation Patterns. Amsterdam; Philadelphia: John Benjamins. 
Filipović, L. (2013). "Typology as a Continuum. Intratypological Evidence from English and Serbo-Croatian”. Goschler, Stefanowitsch, 2013, 17-38. https://doi.org/10.1075/hcp.41.01fil.

Filipović, L.; Ibarretxe-Antuñano, I. (2015). “Motion”. Dabrowska, E.; Divjak, D. (eds), Handbook of Cognitive Linguistics. Berlin; Boston: Walter de Gruyter, 527-46. https://doi.org/10.1515/9783110292022-026.

Gallmann, P.; Siller-Runggaldier, H.; Sitta, H. (2007). Sprachen im Vergleich. Deutsch-Ladinisch-Italienisch, Bd. 1. Bozen-Bolzano: Ladin Pedagogic Institute.

Glynn, D. (2014). "The Many Uses of Run. Corpus Methods and Socio-Cognitive Semantics". Glynn, D.; Robinson, J.A. (eds), Corpus Methods in Cognitive Semantics. Quantitative Studies in Polysemy and Synonymy. Amsterdam; Philadelphia: John Benjamins, 117-44. https://doi.org/10.1075/ hcp.43.05gly.

Goschler, J.; Stefanowitsch, A. (eds) (2013). Variation and Change in the Encoding of Motion Events. Amsterdam; Philadelphia: John Benjamins.

Gries, S. (2006). "Corpus-Based Methods and Cognitive Semantics. The Many Senses of to Run". Gries, S.; Stefanowitsch, A. (eds), Corpora in Cognitive Linguistics. Corpus-Based Approaches to Syntaxand Lexis. Berlin; New York: Mouton de Gruyter, 57-99. https://doi.org/10.1515/9783110197709.57.

Hack, F.M. (2011). "Alcuni tratti sintattici particolari delle varietà retoromanze. Influssi del tedesco, costruzioni romanze oppure sviluppi paralleli di lingue confinanti?". Breu, W. (ed.), L'influsso dell'italiano sul sistema del verbo delle lingue minoritarie. Resistenza e mutamento nella morfologia e nella sintassi = Conference Proceedings (Konstanz, 10-13 December 2008). Bochum: Universitätsverlag Dr. N. Brockmeyer, 185-210.

Hermas, A. (2010). "Language Acquisition as Computational Resetting. Verb Movement in L3 Initial State". International Journal of Multilingualism, 7(4), 343-62. https://doi.org/10.1080/14790718.2010.487941.

Hermas, A. (2015). "The Categorization of the Relative Complementizer Phrase in Third-Language English. A Feature Re-Assembly Account". International Journal of Bilingualism, 19(5), 587-607. https://doi. org/10.1177/1367006914527019.

Hijazo-Gascón, A.; Ibarretxe-Antuñano, I. (2013). "Same Family, Different Paths. Intratypological Differences in Three Romance Languages". Goschler, Stefanowitsch 2013, 39-54. https://doi.org/10.1075/hcp.41.02hij.

Huddleston, R.; Pullum, G.K. (2002). The Cambridge Grammar of the English Language. Cambridge: Cambridge University Press.

lacobini, C.; Masini, F. (2007). "The Emergence of Verb-Particle Constructions in Italian. Locative and Actional Meanings". Morphology, 16(2), 155-88. https://doi.org/10.1007/s11525-๑०6-9101-7.

lacobini, C.; Masini, F. (2009). "I verbi sintagmatici dell'italiano fra innovazione e persistenza. Il ruolo dei dialetti”. Cardinaletti, A.; Munaro, N. (eds), Italiano, italiani regionali e dialetti. Milano: Franco Angeli, 115-35.

Ibarretxe-Antuñano, I. (ed.) (2017). Motion and Space across Languages. Theory and Applications. Amsterdam; Philadelphia: John Benjamins.

Ibarretxe-Antuñano, I.; Hijazo-Gascón, A.; Moret-Oliver, M.-T. (2017). “The Importance of Minority Languages in Motion Event Typology. The Case of Aragonese and Catalan". Ibarretxe-Antuñano 2017, 123-50. https://doi. org/10.1075/hcp.59.06iba. 
Irsara, M. (2015). "Ladin”. Jungbluth, K.; Da Milano, F. (eds), Manual of Deixis in Romance Languages. Berlin: Walter de Gruyter, 140-66.

Irsara, M. (2017). "Promoting Cross-Linguistic Awareness. English Motion Events in a Multilingual Teaching Model". Lingue e Linguaggi, 23, 121-32.

Jessner, U. (2006). Linguistic Awareness in Multilinguals. English as a Third Language. Edinburgh: Edinburgh University Press.

Jessner, U. (2008). "A DST Model of Multilingualism and the Role of Metalinguistic Awareness". The Modern Language Journal, 92(2), 270-83. https://doi. org/10.1111/j.1540-4781.2008.00718.x.

Mayer, M. (1969). Frog, Where Are You? New York: Dial Books for Young Readers.

Pavlenko, A. (ed.) (2017). Thinking and Speaking in Two Languages. Bristol; Buffalo; Toronto: Multilingual Matters.

Rothman, J. (2011). "L3 Syntactic Transfer Selectivity and Typological Determinacy. The Typological Primacy Model". Second Language Research, 27(1), 107-27. https://doi.org/10.1177/0267658310386439.

Rothman, J. (2015). "Linguistic and Cognitive Motivations for the Typological Primacy Model (TPM) of Third Language (L3) Transfer. Timing of Acquisition and Proficiency Considered". Bilingualism. Language and Cognition, 18(2), 179-90. https://doi.org/10.1017/s136672891300059x.

Slabakova, R. (2017). "The Scalpel Model of Third Language Acquisition". International Journal of Bilingualism, 21(6), 651-65. https://doi. org/10.1177/1367006916655413.

Slobin, D.I.; Hoiting, N. (1994). "Reference to Movement in Spoken and Signed Languages. Typological Considerations”. Gahl, S.; Dolbey, A.; Johnson, C. (eds), Proceedings of the Twentieth Annual Meeting of the Berkeley Linguistics Society. General Session Dedicated to the Contributions of Charles J. Fillmore (Berkeley, 18-20 February 1994). Berkeley: Berkeley Linguistics Society, 487-505. https://doi.org/10.3765/bls.v20i1.1466.

Slobin, D.I. (1996a). "Two Ways to Travel. Verbs of Motion in English and Spanish". Shibatani, M.; Thompson, S.A. (eds), Grammatical Constructions. Their Form and Meaning. Oxford: Oxford University Press, 195-220.

Slobin, D.I. (1996b). "From 'Thought and Language' to 'Thinking for Speaking”". Gumperz, J.J.; Levinson, S.C. (eds), Rethinking Linguistic Relativity. Cambridge: Cambridge University Press, 70-96.

Slobin, D.I. (2004). "The Many ways to Search for a Frog. Linguistic Typology and the Expression of Motion Events". Strömqvist, Verhoeven 2004, 219-57.

Spreafico, L. (2009). Problemi di tipologia lessicale. I verbi di moto nello Standard Average European. Roma: Bulzoni Editore.

Strömqvist, S.; Verhoeven, L. (eds) (2004). Relating Events in Narrative. Typological and Contextual Perspectives. New York; London: Psychology Press.

Talmy, L. (1985). "Lexicalization Patterns. Semantic Structure in Lexical Forms". Shopen, Timothy (ed.), Language Typology and Syntactic Description. Cambridge: Cambridge University Press, 57-149.

Talmy, L. (1991). "Path to Realization. A Typology of Event Conflation". Sutton, L.A.; Johnson, C.; Shields, R. (eds), Proceedings of the 17th Annual Meeting of the Berkeley Linguistics Society (Berkeley, 15-18 February 1991). Berkeley: Berkeley Linguistics Society, 480-519. https://doi.org/10.3765/ bls.v17iఠ.1620.

Talmy, L. (2000). Toward a Cognitive Semantics, vol. 2. Cambridge (MA): The MIT Press. 
Talmy, L. (2016). “Properties of Main Verbs”. Cognitive Semantics, 2(2), 133-63. https://doi.org/10.1163/23526416-00202001.

Treffers-Daller, J.; Tidball, F. (2016). "Can L2 Learners Learn New Ways to Conceptualise Events? Evidence from Motion Event Construal among EnglishSpeaking Learners of French". Guiarro-Fuente, P.; Schmitz, K.; Müller, N. (eds), The Acquisition of French in Multilingual Contexts. Bristol: Multilingual Matters, 145-84. https://doi.org/10.21832/9781783094530-009.

Verkerk, A. (2013). "Scramble, Scurry and Dash. The Correlation between Motion Event Encoding and Manner Verb Lexicon Size in Indo-European". Language Dynamics and Change, 3(2), 169-217. https://doi. org/10.1163/22105832-13030202.

Verkerk, A. (2014). "The Correlation between Motion Event Encoding and Path Verb Lexicon Size in the Indo-European Language Family". Folia Linguistica, 35(1), 307-58. https://doi.org/10.1515/flih.2014.009.

Verkerk, A. (2017). "The Goal-over-Source Principle in European Languages. Preliminary Results from a Parallel Corpus Study". Luraghi, S.; Nikitina, T.; Zanchi, C. (eds), Space in Diachrony. Amsterdam; Philadelphia: John Benjamins Publishing Company, 1-40. https://doi.org/10.1075/ slcs.188.01ver.

Von Stutterheim, C.; Carroll, M.; Klein, W. (2009). "New Perspectives in Analyzing Aspectual Distinctions across Languages". Klein, W.; Li P. (eds), The Expression of Time. Berlin; New York: Mouton de Gruyter, 195-216. https:// doi.org/10.1515/9783110199031.195.

Zlatev, J.; Yangklang, P. (2004). "A Third Way to Travel. The Place of Thai in Motion Event Typology”. Strömqvist, Verhoeven 2004, 159-90. 



\title{
EL.LE
}

Vol. 9 - Num. 1 - Marzo 2020

\section{Strategie motivazionali per la classe di lingue Rassegna e confronto delle ricerche empiriche sugli apprendenti}

\author{
Giacomo Cucinotta \\ Università Ca’ Foscari Venezia, Italia
}

\begin{abstract}
Motivation is considered as a pivotal component in successful foreign language learning, and has been the subject of increasing research attention in recent decades. This paper collected and reviewed the literature on motivational strategies in the language classroom. The review focusses on studies that investigate the importance student attached to strategies and juxtaposes the findings. The results confirm that motivation is context-dependent, however some strategies - especially those related to teacher behaviour, creating a positive classroom climate, and recognizing students' efforts - can transfer across contexts, and therefore should be emphasized in future teacher training.
\end{abstract}

Keywords L2 motivation. Motivational strategies. State of the art. SLA. Language teachers education.

Sommario 1 Introduzione.-2 Due approcci alla Motivazione L2. Motivation vs. Motivating. - 3 Strategie motivazionali. - 4 Studi sull'importanza percepita delle strategie motivazionali. - 5 Confrontro tra risultati. L'importanza delle MotS percepita dagli apprendenti. - 6 Conclusioni, limiti e proposte.

\begin{tabular}{ll}
\multicolumn{2}{c}{ Peer review } \\
$\begin{array}{ll}\text { Submitted } & 2019-12-02 \\
\text { Accepted } & 2020-04-23 \\
\text { Published } & 2020-07-30\end{array}$
\end{tabular}

\section{Open access}

(c) 2020 | ()( Creative Commons Attribution 4.0 International Public License 


\section{Introduzione}

Tra i fattori che possono influire sullo studio di una lingua, la motivazione ricopre un ruolo primario nel determinare il successo o il fallimento di un processo di apprendimento, trascendendo altri fattori come le differenze individuali degli apprendenti, gli obiettivi didattici o le risorse a disposizione (Dörnyei, Ryan 2015; Dörnyei 2018; Lamb 2017).

Da un punto di vista didattico, la motivazione è vista come una risorsa sulla quale è possibile agire in modo sistematico per favorire l'apprendimento linguistico (Guilloteaux, Dörnyei 2008; Bernaus, Wilson, Gardner 2009; Papi, Abdollahzadeh 2012; Moskovsky et al. 2013; Alrabai 2016), pertanto riuscire ad agire sulla motivazione degli apprendenti - saper suscitarla, aumentarla, mantenerla e difenderla da fattori negativi - diviene un'abilità centrale all'interno del bagaglio di competenze del docente di lingue.

Nonostante queste premesse, l'uso della motivazione per l'apprendimento linguistico (da qui Motivazione L2) viene lasciato alla sensibilità del singolo docente 0 alla maturazione di esperienze personali fatte sulla propria pelle - e su quella dei propri studenti - senza che vi sia una formazione specifica (cf. Cucinotta 2018; Waddington 2017) che possa fornire non solo aspetti teorici, ma anche indicazioni pratiche utili ad orientarsi nella gestione motivazionale della classe.

Negli ultimi vent’anni il numero delle ricerche sugli aspetti pratici della Motivazione L2 sono andati via via aumentando e in questa rassegna si cercherà di offrire una panoramica degli studi empirici che finora si sono concentrati sulle strategie motivazionali per l'apprendimento linguistico (L2 Motivational Strategies o MotS), ponendo particolare attenzione alle indagini sviluppate sulla percezione che gli apprendenti di lingue straniere hanno dell'importanza delle diverse strategie.

\section{Due approcci alla Motivazione L2. Motivation vs. Motivating}

L'origine della ricerca sulla Motivazione L2 coincide con gli studi sulle comunità di parlanti svolti dallo psicologo Robert Gardner (Gardner 2010; Dörnyei, Ushioda 2011), il quale rifiutava un approccio didattico all'apprendimento di una lingua affermando che

The learning of a second (or foreign) language in the school situation is often viewed as an educational phenomenon [...], such a perception is categorically wrong. (cit. in Au 1988, 75)

Anche per questa ragione, i primi tre decenni di studio della Motivazione L2 (1959-89) hanno visto sviluppare quasi solo ricerche in ot- 


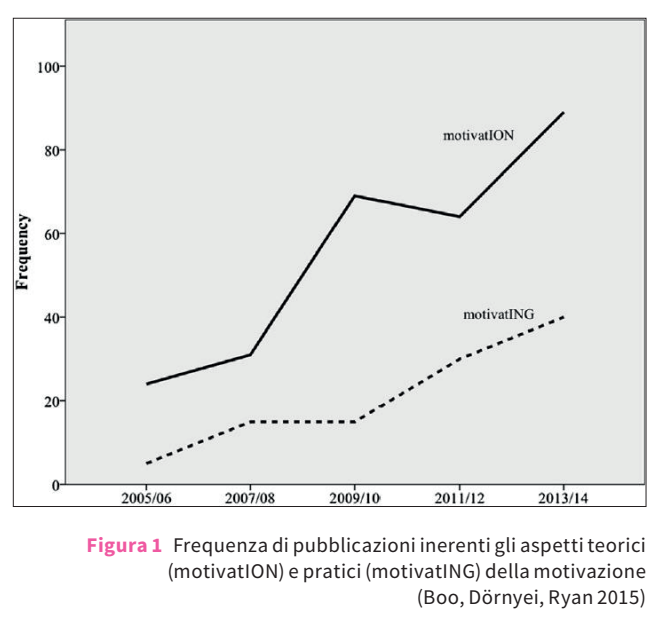

tica strutturalista, il cui fine era individuarne il funzionamento e i fattori costituenti attraverso l'applicazione di schemi provenienti da altri studi in ambito psicologico.

Questo approccio teorico non forniva supporto alla didattica delle lingue e i docenti potevano contare solo su «unsystematic 'bag-oftricks' approaches or on advice coming from questionable theorising» (cit. in Dörnyei 1998, 130).

A partire dalla prima metà degli anni Novanta, tra alcuni accademici emerge il desiderio di ampliare il protocollo di ricerca (Crookes, Schmidt 1991; Oxford, Shearin 1994), non solo per riagganciarsi a nuove teorie psicologiche sulla motivazione, ma anche per includere un nuovo punto di vista rivolto al microcontesto della classe, all'uso di tecniche e materiali didattici e al loro effetto motivazionale sugli apprendenti (Skehan 1991; Dörnyei 1994). Questa nuova attenzione alle pratiche motivazionali diede il via ad una quantità di ricerche sempre maggiore. Boo, Dörnyei e Ryan (2015) riportano come dal 2005 al 2015 il numero delle pubblicazioni sulla Motivazione L2 sia quadruplicato, ma anche come un terzo di queste riguardi l'applicazione di tecniche motivazionali in classe [fig. 1].

\section{Strategie motivazionali}

Quelle tecniche didattiche attuate col fine di contribuire a creare, sostenere, aumentare o mantenere la motivazione dell'apprendente di lingue sono dette strategie motivazionali (MotS) (Dörnyei 2001, 28; Dörnyei, Ushioda 2011, 103).

Prima che le MotS iniziassero ad essere oggetto di ricerche empiriche esistevano già dei lavori che presentavano cataloghi di tec- 
niche didattiche per agire sulla motivazione degli apprendenti; alcuni di questi sono elencati da Dörnyei (1994), ${ }^{1}$ il quale presenta a sua volta una lista di un centinaio di strategie secondo un'ottica «education-friendly» (Dörnyei 1994, 283) che mira a fornire indicazioni didattiche ai docenti. Queste, come le precedenti strategie, derivano dall'esperienza e dal buon senso dell'autore e hanno pertanto solo una valenza aneddotica e non scientifica. Proprio questo limite sarà sottolineato da Gardner, il quale inviterà a testare empiricamente le strategie in un reale contesto d'apprendimento (Gardner, Tremblay 1994a, 364).

Il filone di ricerca empirica sulle MotS parte dalla risposta a questa richiesta, quando Dörnyei deciderà di rendere più sintetico l'elenco di strategie ${ }^{2}$ andando ad analizzare la percezione di 116 docenti d'inglese in Ungheria (Dörnyei, Csizér 1998). Tale studio, oltre ad individuare un primo 'decalogo' di macrostrategie ${ }^{3}$ motivazionali, sarà d'importanza fondante poiché fornirà un esempio di questionario con elenco sistematico di 51 strategie (derivanti da quelle proposte in Dörnyei 1994) nonché un protocollo di ricerca empirica sulle strategie motivazionali che sarà ripreso da decine di altre indagini nei decenni successivi. La ricerca ungherese sarà anche d'ispirazione a Dörnyei per la compilazione di una ricca raccolta di MotS (Dörnyei 2001) che persegue ancora finalità education-friendly, ma nonostante questo, resterà uno studio isolato cui non seguiranno ulteriori validazioni in contesti culturali, etnolinguistici o istituzionali differenti, come invece chiesto dagli autori (Dörnyei, Csizér 1998, 224).

Un rinnovato interesse per le strategie motivazionali comincerà a mostrarsi solo a seguito delle ricerche sviluppate da due dottorande dello stesso Dörnyei e svolte a Taiwan (Cheng, Dörnyei 2007) e in Corea del Sud (Guilloteaux, Dörnyei 2008), dando via ad un numero sempre maggiore non solo di repliche della prima ricerca ungherese, ma anche di ricerche su nuovi e diversi aspetti delle MotS, così che il numero di ricerche in lingua inglese pubblicate nel primo decennio successivo allo studio ungherese (1999-2009) sarà quasi sestuplicato nel decennio successivo (2009-19) passando da 9 a 53 pubblicazioni.

Su 65 studi sulle MotS raccolti per questa rassegna, oltre la metà $(\mathrm{N}=38)$ si pone come obiettivo di indagare la percezione che i docenti o gli studenti (e in alcuni casi entrambi) hanno delle strategie motivazionali, sia in termini di importanza/efficacia $(\mathrm{N}=15)$ sia di

1 In particolare sono citati gli articoli di Keller (1983) e Brophy (1987) ed il volume Teaching by Principles di Brown (2001), la cui prima edizione risale proprio al 1994.

2 Un primo tentivo di riduzione delle categorie in un più conciso 'decalogo motivazionale' è stato proposto in Dörnyei 1996, pur senza alcuno studio empirico alla base.

3 Con macrostrategia si intende una categoria di MotS affini raggruppate attraverso metodi statistici, logici o misti. 
frequenza d'uso $(\mathrm{N}=12)$ o entrambi $(\mathrm{N}=11)$, perlopiù seguendo il solco tracciato da Dörnyei e colleghi. I restanti 26 studi indagano altri aspetti delle MotS, come l'influenza che queste possono avere sulla motivazione o sui risultati degli apprendenti o sulla motivazione dei docenti, ma anche l'analisi di aspetti demotivanti dell'apprendimento linguistico, o studi di caso su particolari contesti.

\section{Studi sull'importanza percepita delle strategie motivazionali}

Dei 26 studi che indagano l'importanza percepita delle MotS, quattordici (14) tengono conto esclusivamente dell'opinione dei docenti, sei (6) indagano esclusivamente il punto di vista dello studente, e sei (6) prendono in considerazione entrambi i pareri.

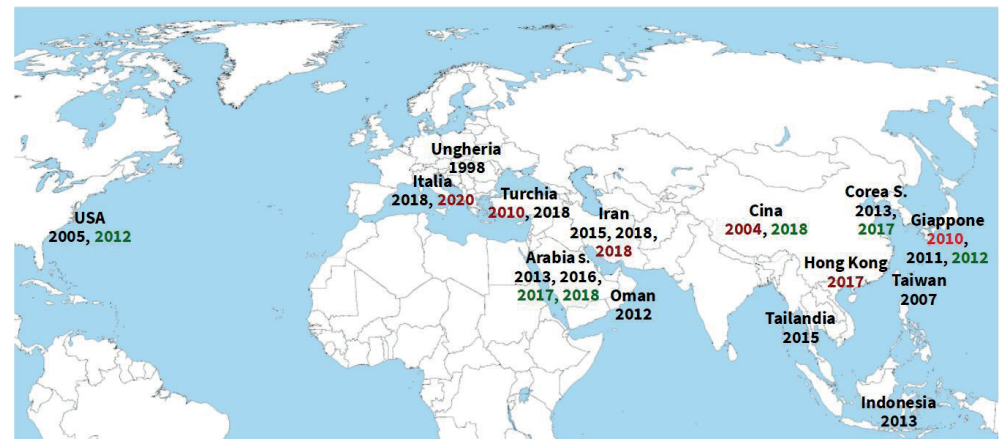

Figura 2 Distribuzione degli studi sull'importanza percepita delle MotS. In nero quelli che indagano l'opinione dei docenti, in rosso quelli che indagano l'opinione degli student e in verde quelli che prendono in considerazione entrambi i pareri

Nella figura 2 è possibile avere una visione d'insieme sulla distribuzione geografica di queste ricerche. Ciò che salta subito all'occhio è la concentrazione della maggior parte degli studi in due macroregioni: l'Asia Pacifica (11 studi) e il Medio Oriente (10 studi). Oltre a queste, altri due studi hanno avuto luogo negli Stati Uniti d'America e solo due (escludendo quello iniziale di Dörnyei e Csizér) in Europa. ${ }^{4} \mathrm{La}$ richiesta di validare lo studio del 1998 in contesti culturali ed etnolinguistici diversi sembra essere stata accolta, ma non ci sono state molte occorrenze di validazioni in contesti simili a quello originale.

Inoltre quasi tutte le ricerche hanno indagato solo l'apprendimento dell'inglese come lingua straniera (EFL) in contesti monolingue e mo-

4 Per i dettagli degli studi, si veda la tab. 1. 
noculturali in cui gli apprendenti hanno poche possibilità di contatto coi parlanti madrelingua e solo in poche eccezioni sono stati coinvolti anche apprendenti o docenti di altre lingue straniere. ${ }^{5}$

Nella tabella 1 sono riassunte, in ordine cronologico, le ricerche sull'importanza percepita delle strategie motivazionali. Oltre ai Paesi in cui si sono svolte, è indicato il tipo di ricerca, il numero di studenti e/o di docenti partecipanti, la tipologia di contesto d'apprendimento/insegnamento, le lingue oggetto di studio e infine il numero di item del questionario e le fonti sulle quali è basato.

Come si può notare, la maggioranza delle ricerche è stata costruita sul modello del questionario di Dörnyei e colleghi (Dörnyei, Csizér 1998; Dörnyei 2001; Cheng, Dörnyei 2007) e solo in pochi casi le fonti da cui è stato sviluppato il questionario non sono citate (Bell 2005; Lu 2018) o hanno origine diversa (Sugita, Takeuchi 2010; Hapsari 2013). In tre casi (Manning, Henneberry, Kobayashi 2012; Shousha 2018; Tavakoli, Yaghoubinejad, Zarrinabadi 2018) i questionari non proponevano un elenco di MotS, bensì chiedevano una valutazione su intere macrostrategie.

Tabella 1 Studi sull'importanza percepita della MotS. In viola gli studi che indagano l'opinione dei docenti, in giallo quelli che indagano l'opinione degli apprendenti e in verde quelli che indagano entrambe. [IMP = Studi sull'importanza delle MotS, IMP+FR = Studi su importanza e frequenza d'uso delle MotS]

\begin{tabular}{|c|c|c|c|c|c|c|c|c|}
\hline Autori & Anno & Paese & Ricerca & Stud & Doc & Contesto & Lingue & Fonti questionario \\
\hline $\begin{array}{l}\text { Dörnyei, } \\
\text { Csizér }\end{array}$ & 1998 & Ungheria & IMP & & 116 & vari & $\mathrm{EFL}$ & 51 MotS < Dörnyei 1994 \\
\hline You & 2004 & Cina & IMP & 130 & & vari & EFL & 43 MotS < Dörnyei 2001 \\
\hline Bell & 2005 & USA & IMP & & 457 & Università & $\begin{array}{l}\text { Fra, Spa, } \\
\text { Ted }\end{array}$ & 80 MotS $<$ fonti non specificate \\
\hline $\begin{array}{l}\text { Cheng, } \\
\text { Dörnyei }\end{array}$ & 2007 & Taiwan & IMP & & 176 & vari & $\mathrm{EFL}$ & $\begin{array}{l}48 \text { MotS < Dörnyei, Csizér } 1998 \\
\text { + Dörnyei } 2001\end{array}$ \\
\hline Deniz & 2010 & Turchia & $\mathrm{IMP}+\mathrm{FR}$ & 179 & & Università & $\mathrm{EFL}$ & 48 MotS < Cheng, Dörnyei 2007 \\
\hline $\begin{array}{l}\text { Sugita, } \\
\text { Takeuchi }\end{array}$ & 2010 & Giappone & IMP & 190 & & Secondaria & EFL & Sugita 2007 \\
\hline $\begin{array}{l}\text { Narikawa, } \\
\text { Okazaki }\end{array}$ & 2011 & Giappone & IMP & & 86 & Secondaria & EFL & 50 MotS < D\&C 1998 \\
\hline $\begin{array}{l}\text { Al-Mahrooqi, } \\
\text { Abrar-Ul- } \\
\text { Hassan, } \\
\text { Asante }\end{array}$ & 2012 & Oman & IMP+FR & & 286 & vari & $\mathrm{EFL}$ & 48 MotS < C\&D 2007 \\
\hline $\begin{array}{l}\text { Manning, } \\
\text { Henneberry, } \\
\text { Kobayashi }\end{array}$ & 2012 & Giappone & $\mathrm{IMP}+\mathrm{FR}$ & 70 & 2 & Università & $\mathrm{EFL}$ & $\begin{array}{l}35 \text { macrostrategie < Dörnyei } \\
2001\end{array}$ \\
\hline
\end{tabular}

5 Nello specifico, gli studi americani di Bell 2005, Ueno 2005 e Ruesch, Bown, Dewey 2012, quelli cinesi di Wang 2010 e Lu 2018 e quello italiano di Cucinotta 2018, che include anche docenti di italiano L2. 
Giacomo Cucinotta

Strategie motivazionali per la classe di lingue

\begin{tabular}{|c|c|c|c|c|c|c|c|c|}
\hline Autori & Anno & Paese & Ricerca & Stud & Doc & Contesto & Lingue & Fonti questionario \\
\hline $\begin{array}{l}\text { Ruesch, Bown, } \\
\text { Dewey }\end{array}$ & 2012 & USA & IMP & 126 & 30 & Università & Varie & $\begin{array}{l}49 \text { MotS < D\&C 1998+ Dörnyei } \\
2001\end{array}$ \\
\hline Guilloteaux & 2013 & Corea S. & IMP+FR & & 268 & Secondaria & EFL & 48 MotS < C\&D 2007 \\
\hline Hapsari & 2013 & Indonesia & IMP & & 28 & Secondaria & EFL & nessuna MotS specifica \\
\hline $\begin{array}{l}\text { Moskovsky } \\
\text { et al. }\end{array}$ & 2013 & Arabia S. & IMP & & 119 & vari & EFL & 53 MotS < D\&C 1998+ C\&D 2007 \\
\hline $\begin{array}{l}\text { Ghadiri, } \\
\text { Vahdani }\end{array}$ & 2015 & Iran & IMP & & 80 & vari & EFL & 48 MotS < C\&D 2007 \\
\hline $\begin{array}{l}\text { Wang, } \\
\text { Vibulphol }\end{array}$ & 2015 & Tailandia & IMP & & 38 & vari & EFL & 48 MotS < C\&D 2007 \\
\hline Alrabai & 2016 & Arabia S. & IMP & & 204 & vari & EFL & $\begin{array}{l}62 \text { MotS da D\&C 1998+ Dörnyei } \\
2001+\text { C\&D 2007+ Moskovsky } \\
\text { et al. } 2013\end{array}$ \\
\hline $\begin{array}{l}\text { Alshehri \& } \\
\text { Etherington }\end{array}$ & 2017 & Arabia S. & IMP & 353 & 105 & Università & EFL & $\begin{array}{l}65 \text { Mots da Dörnyei } 2001+C \& D \\
2007\end{array}$ \\
\hline Wong, Wong & 2017 & Hong Kong & IMP & 150 & & Secondaria & EFL & $\begin{array}{l}48 \text { MotS < Dörnyei 2001+ D\&C } \\
1998\end{array}$ \\
\hline Yeo & 2017 & Corea S. & IMP & 220 & 78 & Università & EFL & 48 MotS < C\&D 2007 \\
\hline Cucinotta & 2018 & Italia & IMP & & 101 & vari & $\begin{array}{l}\text { Varie + } \\
\text { EFL }\end{array}$ & 47 MotS < C\&D 2007 \\
\hline Lu & 2018 & Cina & IMP & 259 & 31 & Università & Varie & 26 Mots $<$ fonti non specificate \\
\hline Safdari & 2018 & Iran & IMP & 168 & & Università & EFL & 48 MotS < C\&D 2007 \\
\hline Shousha & 2018 & Arabia S. & IMP & 165 & 44 & Università & EFL & $\begin{array}{l}\text { Le prime } 10 \text { macrostrategie di } \\
\text { D\&C } 1998\end{array}$ \\
\hline $\begin{array}{l}\text { Tavakoli, } \\
\text { Yaghoubinejad, } \\
\text { Zarrinabadi }\end{array}$ & 2018 & Iran & IMP+FR & & 185 & vari & EFL & $\begin{array}{l}\text { Le } 10 \text { macrostrategie di C\&D } \\
2007\end{array}$ \\
\hline Uştuk & 2018 & Turchia & IMP & & 52 & Università & EFL & 48 MotS < C\&D 2007 \\
\hline Cucinotta & $\begin{array}{l}\text { in corso } \\
\text { di } \\
\text { stampa }\end{array}$ & Italia & IMP & & 162 & Secondaria & $\begin{array}{l}\text { EFL+ } \\
\text { Spa }+ \\
\text { Fra }\end{array}$ & 50 MotS < C\&D 2007 \\
\hline
\end{tabular}

Per quanto riguarda l'analisi dei risultati, in 19 studi su 25 sull'importanza percepita, gli autori optano per l'accorpamento delle MotS in cluster detti macrostrategie. Queste sono state utilizzate inizialmente per fornire un elenco sintetico delle strategie ritenute più importanti, ma oltre a questo, hanno reso più semplice il confronto tra ricerche anche molto diverse tra loro.

In Dörnyei, Csizér (1998) le MotS sono raggruppate in macrostrategie attraverso un'analisi della consistenza interna dei cluster in base all'alfa di Cronbach (Dörnyei 2007, 206-7), ma solo pochi studi [tab. 2] gli autori esplicitano un metodo di raggruppamento basato sulla consistenza interna combinata ad ulteriori metodi come l'analisi fattoriale o il raggruppamento in base ad ambiti concettuali; negli altri casi la scelta è stata meno rigorosa e si è limitata ricalcare le medesime macrostrategie degli studi precedenti, col notevole vantaggio di poter operare confronti più semplici, ma perdendo proprio quelle sfumature peculiari del contesto d'insegnamento/apprendimento analizzato. 
Giacomo Cucinotta

Strategie motivazionali per la classe di lingue

Tabella 2 Metodo di raggruppamento in macrostrategie negli studi sulle MotS

\begin{tabular}{|c|c|c|c|}
\hline $\begin{array}{l}\text { Macrostrategie } \\
\text { ottenute in base } \\
\text { all'alfa di Cronbach }\end{array}$ & $\begin{array}{l}\text { Macrostrategie simili a } \\
\text { Dörnyei, Csizér } 1998\end{array}$ & $\begin{array}{l}\text { Macrostrategie simili } \\
\text { a Cheng, Dörnyei } 2007\end{array}$ & Gruppi prestabiliti \\
\hline $\begin{array}{l}\text { Dörnyei, Csizér } 1998 \\
\text { Cheng, Dörnyei } 2007 \\
\text { You } 2004 \\
\text { Guilloteaux } 2013 \\
\text { Ghadiri, Vahdani } 2015 \\
\text { Cucinotta 2018, in corso } \\
\text { di stampa }\end{array}$ & $\begin{array}{l}\text { Narikawa, Okazaki } 2011 \\
\text { Ruesch, Bown, Dewey } \\
2012\end{array}$ & $\begin{array}{l}\text { Deniz } 2010 \\
\text { Al-Mahrooqi, Abr-ul- } \\
\text { Hassan, Asante } 2012 \\
\text { Wang, Vibulphol } 2015 \\
\text { Wong, Wong } 2017 \\
\text { Yeo } 2017 \\
\text { Safdari } 2018\end{array}$ & $\begin{array}{l}\text { Manning, Henneberry, } \\
\text { Kobayashi } 2012 \\
\text { Alshehri, Etherington } 2017 \\
\text { Shousha } 2018 \\
\text { Tavakoli, Yaghoubinejad, } \\
\text { Zarrinabadi } 2018\end{array}$ \\
\hline
\end{tabular}

Gli studi mancanti di Bell 2005; Sugita, Takeuchi 2010; Moskovsky et al. 2013; Hapsari 2013; Alrabai 2016; Lu 2018 e Uştuk 2018 non hanno operato alcun raggruppamento delle strategie indagate.

\section{Confrontro tra risultati. L'importanza delle Mots percepita dagli apprendenti}

Come già accennato, il raggruppamento in cluster permette di operare un confronto fra i vari studi in modo più semplice rispetto a quello per singole MotS.

La tabella 3 presenta un raffronto delle posizioni delle diverse macrostrategie in quegli studi che hanno esaminato il punto di vista dell'apprendente. Nonostante si tratti di una tabella piuttosto densa, grazie all'uso dei colori è possibile far notare come alcune categorie occupino le medesime posizioni anche in contesti molto distanti tra loro. 
Giacomo Cucinotta

Strategie motivazionali per la classe di lingue

Tabella 3 Riassunto dei risultati degli studi sulla percezione che gli studenti hanno dell'importanza delle MotS

\begin{tabular}{|c|c|c|c|c|c|c|c|c|c|}
\hline & $\begin{array}{l}\text { You } \\
2004\end{array}$ & $\begin{array}{c}\text { Deniz } \\
2010\end{array}$ & $\begin{array}{c}\text { Ruesch et } \\
\text { al. } 2012\end{array}$ & \begin{tabular}{|c|} 
Alshehri, \\
Etherington \\
2017
\end{tabular} & $\begin{array}{l}\text { Yeo } \\
2017\end{array}$ & $\begin{array}{l}\text { Wong, } \\
\text { Wong } \\
2017\end{array}$ & $\begin{array}{c}\text { Safdari } \\
2018\end{array}$ & \begin{tabular}{|c|} 
Shousha \\
2018
\end{tabular} & $\begin{array}{c}\text { Cucinotta } \\
\text { in corso } \\
\text { di stampa }\end{array}$ \\
\hline $\begin{array}{l}\text { Comportamento } \\
\text { DOCENTE }\end{array}$ & 6 & 1 & 2 & 4 & 2 & 2 & 1 & 1 & 1 \\
\hline Creare CLIMA & 1 & 3 & 5 & 3 & 3 & 1 & 3 & 4 & 1 \\
\hline $\begin{array}{l}\text { Riconoscere } \\
\text { IMPEGNO }\end{array}$ & & 5 & 8 & 5 & 1 & 3 & 2 & & 2 \\
\hline $\begin{array}{l}\text { Promuovere } \\
\text { FIDUCIA }\end{array}$ & 5 & 6 & 3 & 2 & 4 & 4 & 4 & 2 & 2 \\
\hline $\begin{array}{l}\text { CONSEGNE } \\
\text { appropriate }\end{array}$ & & 2 & 1 & 7 & 6 & 6 & 5 & 5 & \\
\hline $\begin{array}{l}\text { Attività } \\
\text { INTERESSANTI }\end{array}$ & & 4 & 4 & & 5 & 5 & 8 & 6 & 6 \\
\hline $\begin{array}{l}\text { Promuovere } \\
\text { AUTONOMIA }\end{array}$ & & 7 & 7 & 6 & 8 & 7 & 9 & 7 & \\
\hline $\begin{array}{l}\text { Orientare agli } \\
\text { OBIETTIVI }\end{array}$ & & 8 & 10 & 8 & 7 & 9 & 6 & 7 & 4 \\
\hline $\begin{array}{l}\text { Familiarizzare } \\
\text { coi VALORI L2 }\end{array}$ & 3 & 10 & 6 & 9 & 9 & 10 & 7 & 10 & 3 \\
\hline $\begin{array}{l}\text { Coesione del } \\
\text { GRUPPO }\end{array}$ & 7 & 9 & 9 & 10 & 10 & 8 & 10 & & 5 \\
\hline
\end{tabular}

Nel dettaglio, richiama l'attenzione la posizione delle macrostrategie inerenti il comportamento del docente, la creazione di un clima positivo all'interno della classe, il riconoscimento dell'impegno profuso dall'apprendente e il lavoro di promozione della fiducia in sé dello studente; queste, con poche eccezioni, si trovano sempre nella metà alta delle classifiche. Tali risultati sono simili (e a loro volta confrontabili) con quelli ottenuti negli studi che raccoglievano l'opinione dei docenti (Lamb 2017, 309; Cucinotta 2018, 455). Allo stesso modo, altre macrostrategie occupano quasi sempre le ultime posizioni; è il caso di quelle inerenti la proposta di lavori di gruppo, l'attenzione ai valori inerenti la lingua appresa (di tipo culturale, integrativo, strumentale ecc.), l'orientamento agli obiettivi e la promozione dell'autonomia dell'apprendente.

\section{Conclusioni, limiti e proposte}

Il confronto tra i risultati dei diversi studi sulle MotS permette di ricavare alcune indicazioni operative che possono contribuire alla realizzazione di un sillabo motivazionale per i futuri docenti di lingue.

Se, come dice Dörnyei (2001, 32), qualunque cosa il docente faccia in classe, questa avrà un'influenza motivazionale sugli studenti, allora l'educazione alla consapevolezza di questo strumento didattico dovrebbe costituire una delle basi dei programmi di formazione glottodidattica e non essere affidata solo alla sensibilità e alla buona volontà dell'individuo. 
Per operare scelte didattiche adeguate, oltre alle nozioni teoriche sarebbe utile equipaggiare i futuri docenti con un bagaglio di strategie (validate da prove) con cui possano agire sulla motivazione dei propri apprendenti; per questo la ricerca sulle MotS deve continuare ad ampliarsi, includendo un maggior numero di studi che riguardino lingue diverse dall'inglese (Ueno 2005) e che indaghino nuovi e diversi contesti di apprendimento/insegnamento, come ad esempio quelli che coinvolgono bambini e preadolescenti, i corsi rivolti agli adulti, la differenza tra studio dell'inglese e quello di altre lingue, i corsi L2, sia per adulti sia per figli di immigrati ecc.

Come già indicato poco sopra, le metodologie utilizzate per ottenere dei cluster di MotS sono molto varie (dalla determinazione a priori all'analisi fattoriale); pertanto, sebbene questi risultati mostrino tendenze comuni, è comunque importante operare un confronto - quando possibile - anche tra le singole MotS all'interno dei diversi cluster, tenendo conto del contesto. Come evidenziato da Lamb (2017, 310), quello che un docente iraniano intende con «act naturally in front of students» potrebbe differire (e anche molto) dall'idea che potrebbe farsene un docente cinese o statunitense.

Pertanto, per riuscire a delineare le basi di una proposta formativa sulla motivazione rivolta ai futuri docenti di lingue, la ricerca sulle MotS dovrà porre maggiore attenzione agli aspetti peculiari di ciascun contesto di apprendimento, opponendosi alla tendenza emersa negli studi più recenti di applicare le medesime strategie in $\mathrm{Pa}$ esi e contesti anche molto diversi tra loro e puntare invece alla localizzazione della ricerca, andando ad individuare anche strategie motivazionali tipiche del nuovo ambito di studio e arricchendo il catalogo delle MotS.

\section{Bibliografia}

Al-Mahrooqi, R.; Abrar-ul-Hassan, S.; Asante, C.C. (2012). «Analyzing the Use of Motivational Strategies by EFL Teachers in Oman». Malaysian Journal of ELT Research, 8(1), 36-76.

Al-Mahrooqi, R.; Denman, C.J.; Al-Hasani, F. (2017). «A Student Perspective on the Use of Motivational Strategies by Their English Language Literature Instructors in an Omani University». Language and Culture, 9, 85-103. https://doi.org/10.17223/24109266/9/11.

Alrabai, F. (2011). «Motivational Instruction in Practice. Do EFL Instructors at King Khalid University Motivate Their Students to Learn English as a Foreign Language?». Arab World English Journal, 2(4), 257-85.

Alrabai, F.h (2014). «Motivational Practices in English as a Foreign Language Classes in Saudi Arabia. Teachers Beliefs and Learners Perceptions». Arab World English Journal, 5(1), 224-46. 
Alrabai, F. (2016). «The Effects of Teachers' In-Class Motivational Intervention on Learners' EFL Achievement». Applied linguistics, 37(3), 307-33. https:// doi.org/10.1093/applin/amu021.

Alshehri, E.; Etherington, S. (2017). «Motivational Strategies. The Perceptions of EFL Teachers and Students in the Saudi Higher Education Context». International Journal of English Language Education, 5(2), 46-82. https:// doi.org/10.5296/ijele.v5i2.11727.

Amenu Terfa, M. (2018). «Assessing the Use of Motivational Strategies in English Language Instruction. The Case of Lafto Secondary School Grade Nine Students in Focus». International Journal of Scientific and Research Publications (IJSRP), 8(5), 27-37. https://doi.org/10.29322/ijsrp.8.5.2018.p7707.

Astuti, S.P. (2013). «Teachers' and Students' Perceptions of Motivational Teaching Strategies in an Indonesian High School Context». The Association for the Teaching of English as a Foreign Language in Indonesia (TEFLIN) Journal, 24(1), 14-31.

Astuti, S.P. (2016). «Exploring Motivational Strategies of Successful Teachers». The Association for the Teaching of English as a Foreign Language in Indonesia (TEFLIN) Journal, 27(1), 1-22. https://doi.org/10.15639/teflinjournal.v27i1/1-22.

Au, S.Y. (1988). «A Critical Appraisal of Gardner's Social-Psychological Theory of Second-Language (L2) Learning». Language learning, 38(1), 75-99. htt-

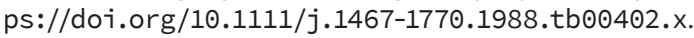

Cucinotta, G. (2018). «Teachers' Perception of Motivational Strategies in the Language Classroom: An Empirical Study on Italian FL and L2 Teachers». EL.LE, 7(3), 447-72. http://doi.org/10.30687/ELLE/2280-6792/2018/03/006.

Cucinotta, G. (in corso di stampa). «Motivare a motivare: strategie motivazionali per gli alunni di lingua straniera nella secondaria di primo grado». Caruana, S.; Chircop, K.; Gauci, P.; Pace, M. (a cura di), Politiche e pratiche per l'educazione linguistica, il multilinguismo e la comunicazione interculturale. Venezia: Edizioni Ca' Foscari.

Bell, T.R. (2005). «Behaviors and Attitudes of Effective Foreign Language Teachers. Results of a Questionnaire Study». Foreign Language Annals, 38(2), 259 70. https://doi.org/10.1111/j.1944-9720.2005.tb02490.x.

Bernaus, M.; Gardner, R.C. (2008). «Teacher Motivation Strategies, Student Perceptions, Student Motivation, and English Achievement». The Modern Language Journal, 92(3), 387-401. https://doi.org/10.1111/j.15404781.2008.00753.x.

Bernaus, M.; Wilson, A.; Gardner, R.C. (2009). «Teachers' Motivation, Classroom Strategy Use, Students' Motivation and Second Language Achievement». Porta Linguarum, 12, 25-36.

Boo, Z.; Dörnyei, Z.; Ryan, S. (2015). «L2 Motivation Research 2005-2014. Understanding a Publication Surge and a Changing Landscape». System, 55, 14757. https://doi.org/10.1016/j.system.2015.10.006.

Brophy, J. (1987). «Synthesis of Research on Strategies for Motivating Students to Learn». Educational Leadership, 45(2), 40-8.

Brown, H.D. (2001). Teaching by Principles. An Interactive Approach to Language Pedagogy. 2nd ed. White Plains (NY): Longman.

Cacho, R.; Frondoso, L. (2018). «Exploring the Motivational Strategies in Second Language Teaching». Dil Eğitimi ve Araştırmaları Dergisi (Rivista di formazione linguistica e Ricerca), 4(3), 229-43. 
Cheng, H.-F.; Dörnyei, Z. (2007). «The Use of Motivational Strategies in Language Instruction. The Case of EFL Teaching in Taiwan». Innovation in Language Learning and Teaching, 1(1), 153-74. https://doi.org/10.2167/ illto48. $\odot$.

Crookes, G.; Schmidt, R.W. (1991). «Motivation. Reopening the Research Agenda». Language Learning, 41(4), 469-512. https://doi. org/10.1111/j.1467-1770.1991.tb๑0690.x.

Deniz, S. (2010). «Student Teachers' Evaluation of the Motivational Strategies Used in Foreign Language Teaching». Social Behavior and Personality, 38(9), 1269-86. https://doi.org/10.2224/sbp.2010.38.9.1269.

Dörnyei, Z. (1994). «Motivation and Motivating in the Foreign Language Classroom». The Modern Language Journal, 78(3), 273-84. https://doi. org/10.2307/330107.

Dörnyei, Z. (1996). «Ten Commandments for Motivating Language Learners». Paper presented at the 30th TESOL convention (Chicago, 26-30 March 1996).

Dörnyei, Z. (1998). «Motivation in Second and Foreign Language Learning». Language Teaching, 31(3), 117-35. https://doi.org/10.1017/ s026144480001315x.

Dörnyei, Z. (2001). Motivational Strategies in the Language Classroom. Cambridge: Cambridge University Press.

Dörnyei, Z. (2007). Research Methods in Applied Linguistics: Quantitative, Qualitative and Mixed Methodologies. Oxford: Oxford University Press

Dörnyei, Z. (2018). «Motivating Students and Teachers». Liontas, John I. (ed.), The TESOL Encyclopedia of English Language Teaching, vol. 7. Alexandria (VA): TESOL, 4293-9.

Dörnyei, Z.; Csizér, K. (1998). «Ten Commandments for Motivating Language Learners. Results of an Empirical Study». Language Teaching Research, 2, 203-29. https://doi.org/10.1191/136216898668159830.

Dörnyei, Z.; Ryan, S. (2015). The Psychology of the Language Learner Revisited. New York: Routledge.

Dörnyei, Z.; Ushioda, E. (2011). Teaching and Researching. Motivation. 2nd ed. London: Routledge.

Gardner, R.C. (2010). Motivation and Second Language Acquisition. The SocioEducational Model. New York: Peter Lang.

Gardner R.C.; Tremblay, P.F. (1994a). "On Motivation, Research Agendas, and Theoretical Frameworks». The Modern Language Journal, 78(3), 359-68. https://doi.org/10.2307/330113.

Gardner, R.C.; Tremblay, P.F. (1994b). «On Motivation, Measurement and Conceptual Considerations». The Modern Language Journal, 78(4), 524-7. https://doi.org/10.1111/j.1540-4781.1994.tb@2073.x.

Ghadiri V.N.; Vahdani S.R. (2015). «Analyzing the Use of Motivational Strategies among Iranian In-Service EFL Teachers». Journal of Education and Management Studies, 5(1), 80-4.

Glas, K. (2016). «Opening Up ‘Spaces for Manoeuvre’. English Teacher Perspectives on Learner Motivation». Research Papers in Education, 31(4), 442-61. https://doi.org/10.1080/02671522.2015.1049287.

Guilloteaux, M.-J. (2013). «Motivational Strategies for the Language Classroom. Perceptions of Korean Secondary School English Teachers». System, 41(1), 3-14. https://doi.org/10.1016/j.system.2012.12.002.

Guilloteaux, M.-J.; Dörnyei, Z. (2008). «Motivating Language Learners. A Classroom-Oriented Investigation of the Effects of Motivational Strategies 
on Student Motivation». TESOL Quarterly, 42(1), 55-77. https://doi. org/10.1002/j.1545-7249.2008.tb00207.x.

Hadfield, J.; Dörnyei, Z. (2013). Motivating Learning. Harlow: Longman.

Hapsari, W. (2013). «Teachers' Perceived Characteristics and Preferences of Motivational Strategies in the Language Classroom». The Association for the Teaching of English as a Foreign Language in Indonesia (TEFLIN) Journal, 24(2), 113-34.

Heinz, M.S.; Kobylinski, C. (2017). «Korean University Students' Perceptions of Teacher Motivational Strategies». International Journal of Learning, Teaching and Educational Research, 16(9), 29-41. https://doi.org/10.26803/ ijlter.16.9.3.

Kakar, S.K.; Pathan, Z.H. (2017). «Exploring the Motivational Strategies Practiced by Pakistani EFL Teachers to Motivate Students in Learning English Language». International Journal of English Linguistics, 7(2), 117-23. https://doi.org/10.5539/ijel.v7n2p117.

Karimi, M.N.; Hosseini Z., S.S. (2018). «Teachers' Use of Motivational Strategies. Effects of a Motivation-Oriented Professional Development Course». Innovation in Language Learning and Teaching, 13(2), 194-204. https://doi. org/10.1080/17501229.2017.1422255.

Keller, J.M. (1983). «Motivational Design of Instruction». Reigelruth, C.M. (ed.), Instructional Design Theories and Models. An Overview of Their Current Status. Hillsdale (NJ): Lawrence Erlbaum, 383-434.

Kikuchi, K. (2009). «Listening to Our Learners' Voices. What Demotivates Japanese High School Students?». Language Teaching Research, 13(4), 453-71. https://doi.org/10.1177/1362168809341520.

Kobylinski, C.; Heinz, M. (2018). «A Qualitative Analysis of Korean Students' Awareness of Motivational Macro Strategies». Journal of Education and Development, 2(3), 1-7. https://doi.org/10.20849/jed.v2i3.469.

Lamb, M. (2017). "The Motivational Dimension of Language Teaching». Language Teaching, 50(3), 301-46. https://doi.org/10.1017/ S0261444817000088.

Lamb, M.; Wedell, M. (2015). "Cultural Contrasts and Commonalities in Inspiring Language Teaching». Language Teaching Research, 19(2), 207-24. https://doi.org/10.1177\%2F1362168814541716.

Lee, T.S.O.; Gardner, D.; Lau, K. (2019). «The Effects of L2 Motivational Strategies. Within and beyond the L2 Classroom». Innovation in Language Learning and Teaching, 13, 1-15. https://doi.org/10.1080/17501229.2019 .1620240.

Lee, T.S.O.; Lin, S.Y. (2015). «A Preliminary Study of the Use of L2 Motivational Strategies in a Business English Course». Asian EFL Journal Quarterly, $17(3), 38-58$.

Li, Q. (2015). «An Intervention Study on the Effectiveness of Motivational Strategy Training in Chinese EFL Context». Asian EFL Journal Quarterly, 17(3), 131-52.

Lu, Y. (2018). «Investigation of Learning Motivation and Motivating Strategies in Less Commonly Taught European Languages Teaching Context in China». Latvijas Universitāte Raksti. Izglītības vadība (Articoli scientifici dell'Università della Lettonia. Gestione della didattica), 817, 72-82. https://doi. org/10.22364/ped.luraksti.817.07.

Madrid, D. (2002). «The Power of the FL Teacher's Motivational Strategies». Cauce, 25, 369-422. 
Maeng, U.; Lee, S.-M. (2015). «EFL Teachers' Behaviour of Using Motivational Strategies. The Case of Teaching in the Korean Context». Teaching and Teacher Education, 46, 25-36. https://doi.org/10.1016/j.tate.2014.10.010.

Manning, C.; Henneberry, S.; Kobayashi, A. (2012). «Comparing Student and Teacher Perceptions of Motivational Teaching Strategies». Shimane Kenritsu Daigaku Sōgō Seisaku Ronsō 島根県立大学総合政策論叢 (Rivista di studi politici dell'Università di Shimane), 23, 75-86.

Moskovsky, C. et al. (2013). «The Effects of Teachers' Motivational Strategies on Learners' Motivation. A Controlled Investigation of Second Language Acquisition». Language Learning, 63(1), 34-62. https://doi.org/10.1111/ j.1467-9922.2012.00717.x.

Muslimin, A.I. (2018). «Lecturers' Motivational Strategy in ESP Classroom». Edulangue, 1(2), 10-23. https://doi.org/10.20414/edulangue.v1i2.285.

Narikawa, T.; Okazaki, H. (2011). «Effective Motivational Strategies for English Classes in Japan. Based on Questionnaires to Teachers and Students». Toyama Daigaku Ningen Hattatsu Kagaku-bu Kiyō 富山大学人間発達科学 部紀要 (Raccolta di saggi della facoltà di sviluppo umano dell'Università di Toyama), 6(1), 157-70.

Oxford, R.L; Shearin, J. (1994). «Language Learning Motivation. Expanding the Theoretical Framework». The Modern Language Journal, 78(1), 12-28. https://doi.org/10.1111/j.1540-4781.1994.tb02011.x.

Papi, M.; Abdollahzadeh, E. (2012). «Teacher Motivational Practice, Student Motivation, and Possible L2 Selves. An Examination in the Iranian EFL Context». Language Learning, 62(2), 571-94. https://doi.org/10.1111/ j.1467-9922.2011.00632.x.

Ruesch, A.; Bown, J.; Dewey, D.P. (2012). «Student and Teacher Perceptions of Motivational Strategies in the Foreign Language Classroom». Innovation in Language Learning and Teaching, 6(1), 15-27. https://doi.org/10.1080 /17501229.2011.562510.

Safdari, S. (2018). «Iranian EFL Learners' Perception of the Importance and Frequency of Teachers' Motivational Strategies». Eurasian Journal of Applied Linguistics, 4(1), 17-25. https://doi .org/10.32601/ejal.460625.

Saleem, M.A.; Ghani, M. (2019). «The Role of Motivational Teaching Strategies Used by English Language Teachers in Urdu Medium Secondary Schools in Pakistan». International Journal of English Linguistics, 9(2), 343-52. https://doi.org/10.5539/ijel.v9n2p343.

Shousha, A.I. (2018). «Motivational Strategies and Student Motivation in an EFL Saudi Context». International Journal of English Language Education, 6(1), 20-44. https://doi.org/10.5296/ijele.v6i1.12535.

Skehan, P. (1991). «Individual Differences in Second Language Learning». Studies in Second Language Acquisition, 13(2), 275-98.

Solak, E.; Bayar, A. (2014). «The Factors Influencing the Motivational Strategy. Use of Non-Native English Teachers». International Journal of Education and Research, 2(2), 1-12.

Soraya, I.; Setiawan, S. (2017). «The Implementation of Teacher's Motivational Strategies in EFL Classrooms». The Asian Conference on Language Learning 2017 = Atti del convegno (Kobe, 11-14 maggio 2017). Nagoya: The International Academic Forum (IAFOR), 257-61. 
Sucuoğlu, E. (2017). «Analysis of Motivational Strategies Used by English Language Teachers Teaching at Secondary Schools». Procedia Computer Science, 120,189-95. https://doi.org/10.1016/j.procs.2017.11.228.

Sugita, M. (2007). «Chūgakkō eigo jugyō ni okeru dōkitsuke hōryaku: kyōshi no shiten kara 中学校英語授業における動機づけ方略：教員の視点から (Strategie motivazionali per le lezioni di inglese nelle scuole medie: dal punto di vista del docente)». Takeuchi, O. et al. (a cura di), Eigo jugyō jissengaku no tenkai 英語授業実践学の展開 (Sviluppo degli studi pratici dei corsi di lingua inglese). Tokyo: Sanseido, 256-66.

Sugita, M.; Takeuchi, O. (2010). «What Can Teachers Do to Motivate Their Students? A Classroom Research on Motivational Strategy Use in the Japanese EFL Context». Innovation in Language Learning and Teaching, 4(1), 2135. https://doi.org/10.1080/17501220802450470.

Sugita McEown, M.; Takeuchi, O. (2014). «Motivational Strategies in EFL Classrooms. How Do Teachers Impact Students' Motivation?». Innovation in Language Learning and Teaching, 8(1), 20-38. https://doi.org/10.1080/17 501229.2012.741133.

Tavakoli, M.; Yaghoubinejad, H.; Zarrinabadi, N. (2018). «Using Motivational Strategies in L2 Classrooms. Does Culture Have a Role?». Current Psychology, 37(3), 477-87. https://doi.org/10.1007/s12144-016-9523-2.

Ueno, J. (2005). «An Analysis of Learner Motivation of Less Commonly Taught Languages». Journal of the National Council of Less Commonly Taught Languages, 2, 45-72.

Uştuk, Ö. (2018). «Turkish EFL Instructors' Perceived Importance of Motivational Strategies. A Descriptive Study». Journal of Foreign Language Education and Technology, 3(1), 215-33.

Vibulphol, J. (2016). «Students' Motivation and Learning and Teachers' Motivational Strategies in English Classrooms in Thailand». English Language Teaching, 9(4), 64-75. https://doi.org/10.5539/elt.v9n4p64.

Waddington, J. (2017). «Teacher Understanding and Implementation of Motivational Strategies in ELT». ELT Journal, 72(2), 162-74. https://doi. org/10.1093/elt/ccx044.

Wang, L.; Vibulphol, J. (2015). «Motivational Strategies for English Classrooms in Thailand. Perspectives of English Teachers». OJED. Online Journal of Education, 10(1), 429-43.

Wang, Y. (2010). «A Survey of the Foreign Language Learning Motivation among Polytechnic Students in China». Journal of Language Teaching and Research, 1(5), 605-13. https://doi.org/10.4304/jltr.1.5.605-613.

Wong, R.M.H. (2014). «An Investigation of Strategies for Student Motivation in the Chinese EFL Context». Innovation in Language Learning and Teaching, 8(2), 132-54. https://doi.org/10.1080/17501229.2013.777449.

Wong, R.M.H.; Wong, R. (2017). «Alignment of Motivational Strategies. The Perceptions of Teachers and Students». Educational Practice and Theory, 39(2), 57-85. https://doi.org/10.7459/ept/39.2.05.

Yeo, K. (2017). «Perceptions on Motivational Strategies in the Korean EFL Setting. Three Voices of Korean Instructors, Native English Speaking Instructors, and Their Learners». Hyeondaemunbeob-yeongu 현대문법연구 (Studio di grammatica moderna), 94, 99-133. https://doi.org/10.14342/ smog.2017.94.99. 
Giacomo Cucinotta

Strategie motivazionali per la classe di lingue

You, C.J.; Dörnyei, Z. (2016). «Language Learning Motivation in China. Results of a Large-Scale Stratified Survey». Applied Linguistics, 37(4), 495-516. https://doi.org/10.1093/applin/amu○46.

You, Z. (2004). «The Role of Motivational Strategies in English Language Learning. An Investigation into the Relationship between the Student Language Achievement Level at Jilin University and Their Motivational Strategies». Teaching English in China. CELEA Journal, 27(3), 70-1. 


\title{
EL.LE
}

Vol. 9 - Num. 1 - Marzo 2020

\section{Canzoni per l'insegnamento linguistico-culturale del tedesco e per la didattica della traduzione}

\author{
Barbara Delli Castelli \\ Università degli Studi «G. d’Annunzio» di Chieti - Pescara, Italia
}

\begin{abstract}
This paper aims to discuss the way in which song translation can be used as an effective way of language/translation teaching, focussing in particular on German as a Foreign Language in higher education at the university level. Starting from some considerations about the distinctive features of song lyrics and their use as a didactic tool in foreign language, culture and literature classes, a reflection is proposed on a way to introduce pop songs also in language/translation classes in relation to a variety of possible translation skopoi.
\end{abstract}

Keywords Foreign language. Culture and literature teaching. Translation teaching. German as a foreign language. Pop song translation. Translation and creative rewriting.

Sommario 1 Introduzione. - 2 La canzone. - 3 Le canzoni nell'educazione linguisticoculturale. -4 Canzoni fra traduzioni e riscritture. -4.1 Tradurre canzoni per la comprensione. - 4.2 Tradurre canzoni per la lettura. - 4.3 Tradurre canzoni per la performance canora. 5 Conclusioni.

\section{Peer review}

Submitted 2019-06-20

Accepted 2020-05-10

Published 2020-07-30

\section{Open access}

(c) 2020 | (9) Creative Commons Attribution 4.0 International Public License 


\section{Introduzione}

Da alcuni decenni nell'insegnamento delle lingue straniere si persegue l'obiettivo di migliorare le pratiche didattiche non solo improntandole allo sviluppo delle abilità comunicative degli apprendenti, ma mettendo anche in rilievo il concetto di interazione fra lingua, cultura e identità. ${ }^{1}$ Le competenze (inter)culturali che ne discendono sono state identificate e definite nel tempo in modi diversi in base a diverse prospettive e a diversi approcci teorici. ${ }^{2}$ Ciononostante il tema 'cultura' resta ancora una questione molto dibattuta fra gli studiosi, con conseguenze nella redazione dei curricula scolastici e universitari, fino alla didassi quotidiana.

A livello accademico si osserva, ad esempio, la tendenza a tenere nettamente separati l'insegnamento della lingua straniera da quello della relativa letteratura, in quanto la Sprachdidaktik, focalizzata su skills comunicative, conversazionale e discorsive, si distingue nettamente dalla Literaturdidaktik, votata piuttosto all'analisi e all'interpretazione di testi letterari. Si tratta, però, di una semplificazione smentita da numerosi studi, i quali hanno dimostrato come l'educazione linguistica e quella letteraria non si possano considerare come

1 A questo proposito Balboni osserva che: «dagli anni Sessanta, con l'inizio della globalizzazione che costringe ad apprendere lingue per scopi comunicativi e non più culturali (con la 'C' maiuscola), la glottodidattica diventa [...] una teoria dell'insegnamento delle lingue-culture, nozione duplice nell'espressione lessicale ma unitaria nella realtà: una cultura esiste in quanto si esprime in una lingua e la lingua è lo strumento di perpetuazione di una data cultura» (Balboni 2013, 61). La cultura, inoltre, è alla base della costituzione d'identità di ciascun parlante in quanto ogni persona nel suo agire opera secondo la propria identità culturale d'appartenenza che acquisisce, definisce, modifica e ridefinisce attraverso un processo che dura tutta quanta l'esistenza (Di Cristofaro Longo 1993, 33-5). Pertanto l'analisi dell'interazione tra identità e cultura/lingua costituisce una scelta necessaria per 'comprendere l'altro', una via privilegiata per individuare percorsi di formazione tali da rendere le persone capaci di un'interazione costruttiva con l'altro.

2 Studi quali quelli di Erdmenger, Istel (1973), Melde (1987), Thürmann (1994) e Bredella (2002) rappresentano, ad esempio, le diverse posizioni che hanno caratterizzato la teoria e la prassi della Fremdsprachenlehre in Germania a partire dagli anni Settanta: quella comunicativo-pragmatica, quella orientata all'educazione politica e quella letterario-interculturale. A partire dai primi anni del XXI secolo si è acceso poi un dibattito accademico pluridisciplinare atto a determinare il fine generale, gli scopi specifici, il campo di applicazione e lo statuto epistemologico del concetto stesso di 'competenza interculturale'. Tale dibattito si sviluppa intorno a due concezioni antitetiche delle quali una fa riferimento alla conoscenza specifica di una o più lingue e culture straniere, una sorta di competenza bi- o policulturale (Herzog 2003), mentre l'altra si appella ad una più universale abilità di gestire situazioni inconsuete, non familiari (Mecheril 2003). Infine, la pubblicazione nel 2001 del Common European Framework of Reference for Languages (CEFRL), contraddistinto da un orientamento pragmatico-orientativo che si pone come obiettivo la 'cittadinanza interculturale', ha influenzato grandemente la riflessione sulla didattica della cultura, inquadrandola in un'ottica transnazionale che caratterizza la ricerca contemporanea. Per una panoramica completa sul tema si veda Snaidero 2008. 
nettamente distinte, pur nelle rispettive autonomie. ${ }^{3}$ Tra le due, infatti, esiste un rapporto di reciproco scambio, in quanto la formazione linguistica è funzionale a quella letteraria, che a sua volta concorre allo sviluppo linguistico complessivo degli apprendenti. ${ }^{4}$

Inoltre, il termine 'cultura' non può esaurirsi esclusivamente nella letteratura, ma deve comprendere anche la storia, la geografia, gli usi e i costumi, il pensiero filosofico, le arti figurative e la musica, nonché forme di espressione multimediale quali cinema, televisione, fumetti ecc. ${ }^{5}$

In quest'ottica le competenze (inter)culturali risultano particolarmente varie e articolate in quanto coinvolgono aspetti culturologici generali e specifici che vanno dai gusti, la mentalità, i bisogni, le attitudini e i valori di un gruppo di parlanti alle sue forme artistiche, con riferimento tanto alla cultura 'alta' quanto alla cultura di massa. ${ }^{6}$

Tali aspetti, inoltre, trovano utilizzo in forme diverse anche nella Translationsdidaktik, alla quale da qualche tempo viene riservato ampio spazio nei curricula universitari. ${ }^{7}$ La traduzione, infatti, partendo da un'accurata analisi del testo fonte, implica una comparazione interlinguistica a tutti i livelli del sistema (grammaticale, lessicale,

3 Sul rapporto fra educazione letteraria ed educazione linguistica si vedano fra gli altri Caon, Spaliviero 2015; Lavinio 2005; Luperini 2013.

4 Se da un lato, infatti, appare evidente l'impossibilità di accostarsi alla complessità di un testo letterario senza un'adeguata formazione linguistica, dall'altro risulta chiaro che l'acquisizione di competenze letterarie offre agli studenti la possibilità di rafforzare le proprie abilità linguistiche (ricettive, produttive e integrate) e di approfondire gli aspetti lessicali, retorici e morfosintattici della lingua oggetto di studio (Spaliviero 2015, 10-11).

5 Negli ultimi anni alcuni lavori sull'acquisizione linguistica hanno aperto la strada all'utilizzo nella lezione di lingua straniera di testi non solo linguistici (immagini, annunci pubblicitari, film, serie televisive, canzoni pop, rock e rap ecc.) per sviluppare negli apprendenti capacità critiche non solo come lettori, ma anche come spettatori e ascoltatori. Si vedano fra gli altri Blell, Kupetz 2010; Gallo, Reeg, Simon 2016; Hecke, Surkamp 2010.

6 In particolare Badstübner-Kizik $(2010,1596)$ attribuisce al concetto di arte legato all'insegnamento delle lingue straniere un significato ampio, che abbraccia tutte le opere prodotte da una società dall'inizio della sua esistenza fino al momento attuale. Pertanto l'arte è intesa come qualsiasi espressione frutto di un processo creativo e, come tale, ogni prodotto di una cultura può trovare spazio nella lezione di lingua.

7 Benché la traduzione - intesa come metodo grammaticale-traduttivo - sia stata bandita per molti decenni dalla didassi delle lingue straniere, da qualche tempo essa gode di un rinnovato interesse da parte degli studiosi. Occorre, però, distinguere la traduzione professionale da quella didattica: la prima si basa su teorie, principi e metodi della Übersetzungswissenschaft e ha come obiettivo la formazione di mediatori linguistici professionali; la seconda, invece, punta piuttosto a una sensibilizzazione linguistica e culturale degli apprendenti che coinvolge il piano morfosintattico, testuale e discorsivo. In questo senso la traduzione a livello accademico non deve essere relegata alla sola formazione dei futuri traduttori professionali, ma può essere introdotta anche in altri percorsi finalizzati più genericamente all'apprendimento linguistico come supporto per la riflessione contrastiva. 
stilistico, pragmatico-culturale), favorendo la riflessione metalinguistica e sollecitando l'apprendente al controllo cognitivo delle operazioni di decodifica e ricodifica verbale, le quali richiedono necessariamente un'opportuna preparazione (inter)culturale. Se poi il testo fonte è un testo letterario o artistico in genere entreranno in gioco anche competenze interpretative e teorico-critiche, spostando il discorso su un piano comparatistico e interculturale polivalente.

Pertanto, a livello accademico, l'analisi di testi (artistici) autentici e l'esplorazione delle possibili strategie traduttive risulteranno particolarmente efficaci tanto per la didattica della lingua e della letteratura quanto per quella della traduzione, ponendo in un'ottica contrastiva le culture coinvolte.

Nel presente saggio ci si occuperà dell'utilizzo delle canzoni in quei corsi universitari nei quali all'apprendimento linguistico e alla landeskundliche Lehre (ivi compresa la letteratura) si lega anche l'attività traduttiva. Partendo da una riflessione complessiva sulle caratteristiche del testo canoro e sul suo possibile impiego, tanto nella lezione di lingua quanto in quella di letteratura, si passa a considerare la canzone come prodotto di una determinata cultura che può anche essere accolto in altri contesti linguisticamente distanti attraverso operazioni di traduzione in senso stretto e/o di 'traduzione creativa'. A livello esemplificativo vengono presentate alcune versioni tedesche di evergreen della musica leggera italiana che hanno riscosso relativo successo anche al di fuori dei confini nazionali.

\section{La canzone}

La canzone è un genere discorsivo declinato in diversi generi musicali (per una tassonomia dei generi musicali si veda Fabbri 2015) che appartiene di norma alla cultura di massa e con il quale ci si confronta quotidianamente attraverso diverse modalità di fruizione. ${ }^{8}$ La sua testualità si articola su un doppio livello, quello verbale - nei suoi diversi aspetti: fonologico/prosodico, lessicale, sintattico e semantico - e quello musicale, fra loro in stretto rapporto dialogico. A questi due livelli si aggiungono una serie di ulteriori aspetti extra-testuali legati all'interpretazione e all'esecuzione del brano (il timbro vocale, l'intonazione e gli accenti dei cantanti e dei musicisti) ciascuno dei quali ha un proprio peso nel processo di significazione (Middleton 1990, 256). La canzone costituisce, pertanto, un evento semiotico complesso nel quale:

8 A questo proposito Kaindl afferma che: «Popular music is part of our daily lives, both in its aural dimension, e.g. in underground stations, shops, and restaurants, and in its visualized form, in TV and in concerts» (Kaindl 2005a, 235). 
la sovrapposizione di parole e musica comporta che sul piano dell'espressione si venga a creare una situazione di "stratificazione multipla" del significante (testo, musica, esecuzione musicale, esecuzione canora), ulteriormente complicata dalla natura del sistema di articolazione della musica. (Garzone 2012, 17)

Nella composizione di un brano canoro due sono le possibili strade da percorrere: partire dal testo verbale musicandolo in un secondo momento oppure partire dal testo musicale aggiungendo successivamente le parole. A seconda del percorso intrapreso il condizionamento reciproco fra testo verbale e testo musicale si presenterà in modi diversi. Nello specifico, quando è la musica a essere ideata per prima, l'elemento linguistico ne risulterà fortemente vincolato, dovendo rientrare obbligatoriamente nella griglia della cosiddetta 'mascherina', che costituisce il disegno guida entro il quale il paroliere deve operare con conseguenze sul piano metrico e prosodico (allungamenti metrici, apocopi, inversioni sintattiche ecc.). Quando invece viene prima composto il testo verbale, sarà la musica a dover assecondare le esigenze delle parole (sulla stesura del testo canoro e le relative criticità si veda Bandini 1996).

La presenza di un testo verbale - oltre a quello musicale - rappresenta uno dei tratti distintivi della canzone rispetto ad altre forme di musica popolare. Tale testo può essere più o meno intelligibile a seconda dei generi: in alcuni (hard-rock, metal, noise ecc.) il livello musicale offre meno spazio alla fruibilità linguistica, mentre in altri (pop, rock melodico, rap ecc.) l'aspetto linguistico e il significato delle parole giocano un ruolo ben più centrale.

Un tratto essenziale della canzone risiede nella ripetitività, la quale può essere 'musematica', ovvero prevedere la ripetizione di elementi melodici portatori di significato ed estrapolabili dal proprio contesto, oppure 'discorsiva', vale a dire la ripetizione di frasi, periodi o sezioni di testo verbale:

the former, therefore, tends towards a one-levelled structural effect, the latter to a hierarchically ordered discourse. (Middleton 1990, 269)

Di norma le canzoni a carattere discorsivo sono organizzate in strofe e ritornelli. Nelle prime viene articolato un discorso, mentre ai secondi è affidato il compito di riassumere o commentare il discorso proposto nelle strofe;

allo stesso tempo il ritornello - quando c'è - si presenta come il culmine musicale di una preparazione avvenuta nel corso della strofa. (Fabbri 2001, 556) 
Nell'ambito della musica di più ampio consumo (musica leggera, popmusic, Schlager-Musik ecc.), i parolieri tendono a proporre un linguaggio quotidiano, vicino al parlato, attingendo a un lessico proprio della comunicazione spontanea (sintassi nominale, inserti dialogici, colloquialismi), accompagnato da un modo di organizzare il discorso che ricalca di norma quello realmente praticato negli spazi sociali. ${ }^{9}$ Non si tratta, però, di una lingua 'viva e vera', ma piuttosto di una lingua 'verosimile', grazie alla quale le canzoni, con il loro potere evocativo, concorrono a costituire un patrimonio linguistico e culturale condiviso,

un serbatoio di memoria collettiva che ci fa sentire tutti, al di là delle differenze regionali, generazionali, sociali, culturali, parte di una medesima comunità. (Coveri 2011, 75)

Infine, da un punto di vista tematico, benché l'argomento privilegiato della musica leggera sia quello dell'amore declinato nelle sue varie sfaccettature, a partire dagli anni Sessanta-Settanta molti artisti scoprono che la canzone può servire anche per far poesia, politica o meglio ancora per esprimere ansie, sogni e speranze della coscienza collettiva. Pertanto, attraverso la musica leggera, oltre a offrire spunti musicali e versetti spensierati, si possono affrontare anche argomenti culturali di valenza universale (famiglia, lavoro, solitudine, amicizia ecc.) o regionale (usi e costumi, episodi di storia nazionale, fatti di cronaca locale ecc.) che possono essere ampiamente condivisi oppure peculiari di singole culture e gruppi sociali.

\section{Le canzoni nell'educazione linguistico-culturale}

In base a quanto finora detto, si ritiene che l'idea di un metodo di educazione linguistica e culturale che contempli anche l'utilizzo di brani canori possa dare avvio a diversi percorsi didattici a seconda del tipo di utenza e del livello di competenza linguistica del gruppo-classe, risultando estremamente motivante tanto per il docente quanto per gli apprendenti.

9 In questo senso anche la deissi svolge un ruolo centrale nella misura in cui molte canzoni mettono in scena un discorso in cui un io si rivolge ad un tu attraverso un riferimento ad uno spazio e ad un tempo generici: «L'here e il there, il now e il then, sono presenti in un gran numero di testi, eppure attraverso il carattere drammatico, il tema (l'argomento) e l'atmosfera musicale del brano, questi avverbi assumono una pregnanza ed un'intensità assolute, al punto di essere in grado di lasciare la materia sonora, il racconto del e nel brano, per abitare nella coscienza e nella vita dell'ascoltatore» (Martino 2004, 193). In particolare sulle caratteristiche della lingua italiana delle canzoni a partire dagli anni Sessanta si vedano Coveri 2011 e De Mauro 1996. 
Guardando alla didattica delle lingue straniere in generale, le canzoni sono da considerarsi come materiali autentici, nei quali la componente verbale ricopre un ruolo fondamentale. Esse, infatti, in quanto testi audio, ben si prestano a esercitare e consolidare la comprensione orale in lingua straniera, oltre che a fornire stimoli relativi alla fonetica, al lessico e alle strutture linguistiche in genere. Le canzoni, inoltre, favoriscono la riflessione su aspetti sociolinguistici (registri linguistici, fraseologismi, regionalismi, espressioni gergali ecc.) e offrono lo stimolo per una comunicazione spontanea relativamente a specifiche tematiche che possono essere introdotte attraverso i brani proposti. ${ }^{10}$

L'utilizzo di musica e canzoni negli spazi didattici, però, non esaurisce la propria funzione nella sola facilitazione all'apprendimento linguistico in senso stretto, ma - al pari dei testi letterari in genere - offre un impulso all'analisi e all'interpretazione testuale. Il testo verbale, infatti, può essere analizzato anche a livello tematico e narrativo, indagando, ad esempio, come autori diversi affrontano gli stessi argomenti utilizzando stili e procedimenti discorsivi differenti. A partire dalla canzone, infatti:

è possibile evidenziare sia gli elementi di somiglianza e di continuità con il testo letterario, sia le differenze costitutive di ogni genere, stabilendo un confronto su più livelli: da quello semantico (ad esempio, sulle modalità di sviluppo dello stesso tema, come l'amore, la guerra, la morte ecc.), a quello espressivo (ad esempio, sull'utilizzo delle figure retoriche come la metafora, la similitudine, l'allitterazione ecc.). Gli echi letterari presenti nelle canzoni, infatti, possono rappresentare un punto di partenza adeguato per avviare allo studio contenutistico e formale delle opere canoniche. (Spaliviero 2015, 20)

10 Sull'uso didattico della canzone in termini di apprendimento significativo della lingua straniera si vedano Caon, Lobasso 2008 e Balboni 2018 
Considerando, dunque, le affinità esistenti fra le canzoni e la letteratura 'alta', ${ }^{11}$ è possibile ipotizzare dei percorsi didattici nei quali dei brani canori di successo possano fungere da trait d'union tra una cultura di massa e la letteratura,

tra esperienza estetica "informale" che i discenti fanno quotidianamente in contesto extrascolastico ed esperienza estetica "formale", vissuta in contesto scolastico. (Caon 2005, 78)

Infine, alle canzoni va riconosciuto un valore che è interculturale e al tempo stesso culturospecifico e che può offrire lo spunto per un proficuo confronto fra la cultura propria e quella straniera (Badstübner-Kizik 2010, 1600-1), in quanto:

Popmusik als globales Phänomen vermag wohl den Eindruck von Vertrautheit zu erwecken und könnte daher Hemmschwellen zur ,anderen' Kultur überwinden helfen. Popmusik ist nicht nur global, sondern durch die Landessprache(n), durch Textinhalte, Musiktradition und Inszenierung der Interpreten auch national oder regional geprägt, was wesentlich ihren ,fremdkulturellen' Aspekt ausmacht, der neugierig machen kann. (Bettermann 2013, 104)

La riflessione (inter)culturale sulle canzoni, pertanto, può avvenire sia a un livello interno al testo verbale (analisi linguistico-tematica, modalità di significazione, convenzioni poetiche ecc.), sia relativamente al rapporto instaurato fra testo verbale e testo musicale e ai relativi condizionamenti (metrica, accenti, apocopi, rime ecc.), sia su un piano extratestuale (interpreti, esecuzione canora e musicale, riconducibilità a un certo genere/tradizione musicale ecc.).

In questo senso si ritiene che anche l'esplorazione delle strategie traduttive relative a testi canori permetta di integrare in modo efficace la riflessione fin qui condotta, spostandola sul piano della mediazione interlinguistico-culturale. Infatti, il confronto fra canzoni in lingua originale e le rispettive versioni in una o più lingue straniere permette di osservare criticamente le modalità di 'esportazione' e di ricezione di un prodotto culturale dall'ambiente originario in nuovi ambienti linguisticamente distanti e diversamente convenzionalizzati, scoprendo che brani e melodie ritenuti patrimonio di un solo Paese hanno in realtà superato i confini nazionali, trovando una propria collocazione in un panorama musicale più vasto e condiviso.

11 A questo proposito Colombo precisa che: «la fruizione di canzoni [...] costituisce pur sempre un'esperienza estetica, non priva di derivazioni tematiche e formali dalla letteratura "alta"» (Colombo 2005, 56). 


\section{Canzoni fra traduzioni e riscritture}

La traduzione delle canzoni può essere ricondotta nella categoria della traduzione letteraria, evidenziando problematiche che sono in larga misura assimilabili a quelle della traduzione del testo poetico. ${ }^{12}$ Quella del testo canoro si propone, però, come una variante 'estrema' della trasposizione poetica, poiché qui il traduttore è chiamato a confrontarsi non solo con il significato, gli artifici espressivi e i parallelismi fonici e semantici del testo verbale, ma anche con i vincoli imposti dalla griglia musicale all'interno della quale esso è collocato. Per le canzoni, dunque, - al pari di altri testi multimediali - si può parlare di una medium-constrained translation, ${ }^{13}$ in cui la trasposizione delle parole è dipendente dal codice musicale sul quale il traduttore ha limitate possibilità di intervento, in quanto:

It is an established fact that, generally, during the process of lyrics translation, the music must not be changed to fit the translated text. That is, music cannot adjust to the rhythm of the words; it is always words that have to be adapted to the rhythm of the music. Therefore, the fact that the source text is embedded in music poses a great deal of problems for the translator, problems that are not to be encountered in other kinds of translation. (Surugiu 2010, 120)

Nel caso delle canzoni, dunque, il compito del traduttore è reso più complesso dalla concomitanza di sistemi intersemiotici che, nella maggioranza dei casi, preclude la possibilità di raggiungere il più alto grado di equivalenza dinamica nel testo di arrivo. ${ }^{14}$ La scelta dell'approccio traduttivo - traduzione letterale o libera oppure riscrittura creativa - dipenderà dunque dalla funzione e dalla situazione d'uso che il metatesto dovrà soddisfa-

12 Entrambi i tipi di testo, infatti, presentano versi strutturati in strofe, la ripetizione di alcune parti (ritornello, parole chiave ecc.), la rilevanza dell'aspetto ritmico (rime, accenti ecc.), nonché una resa originale del linguaggio perseguita attraverso un'accurata selezione e disposizione delle parole.

13 L'espressione è mutuata da Zanettin (1998), che la usa con riferimento alla traduzione dei fumetti. Il concetto di constrained translation è, però, da attribuirsi a Mayoral, Kelly, Gallardo (1988), i quali evidenziano come la traduzione di testi multimediali sia particolarmente complessa poiché l'aspetto verbale è vincolato ad altre forme di espressione (immagini, musica, voce ecc.) che creano limitazioni al traduttore, esercitando una forte influenza sulle sue scelte.

14 A questo proposito si veda la distinzione operata da Nida (1964) tra «equivalenza formale», attenta alle corrispondenze fra forma e contenuto, ed «equivalenza dinamica», che trasmette inalterata al pubblico di arrivo la globalità del significato testuale, determinando un effetto simile a quello prodotto dal testo di partenza sul fruitore originario. 
re nel contesto linguistico-culturale che lo andrà ad accogliere. ${ }^{15}$

Le principali ragioni per cui un brano canoro necessita di traduzione sono sostanzialmente le seguenti:

1. comprendere il significato del testo;

2. leggere il testo tradotto come una poesia;

3. cantare/incidere la canzone in un'altra lingua.

A questi tre punti sono riconducibili le cinque modalità di esportazione individuate da Franzon (2008). ${ }^{16}$ In particolare, se lo skopos traduttivo sarà quello della mera comprensione oppure della lettura del testo tradotto come se fosse una poesia, si sceglierà - con modalità diverse - di operare a livello verbale senza tener conto del codice musicale; se invece una canzone è destinata a un nuovo mercato, essa potrà essere presentata in lingua originale oppure in una lingua diversa. In questo secondo caso sarà necessario stabilire se: a) tradurre il testo, adattando la traduzione alle esigenze della musica; b) tradurre il testo, adattando la musica alla traduzione; ${ }^{17}$ c) scrivere un nuovo testo nella lingua di arrivo.

\subsection{Tradurre canzoni per la comprensione}

Tradurre per comprendere le parole di una canzone è un'attività che, di base, non richiede particolari competenze, se non la conoscenza dei due codici a confronto. Il prodotto finale sarà una Dienstübersetzung, una 'traduzione di servizio', in cui il significato di ogni parola e porzione di testo sarà reso in modo semplice e intellegibile, talvolta anche attraverso il ricorso a forme parafrastiche. Questo approccio traduttivo è legato al desiderio o alla necessità pedagogica di comprendere il testo ed è estraneo alle istanze della 'performabilità' canora, infatti:

15 Un principio basilare della Skopostheorie è quello secondo il quale: «a translation need not necessarily be retrospectively 'equivalent' to a source-text interpretation, but should be prospectively 'adequate' to a target-text skopos» (Vermeer 1996, 77). Pertanto, il fattore che determina le decisioni e le scelte di un traduttore risiede nello scopo stesso per cui è chiamato a tradurre quel testo. Tale principio appare come particolarmente opportuno se applicato alla traduzione delle canzoni per la quale la funzionalità va ravvisata non solo in relazione alla musica, ma anche alla situazione d'uso.

16 Franzon (2008, 376 ss.) distingue fra: 1) leaving the song untranslated; 2) translating the lyrics but not taking the music into account; 3 ) writing new lyrics to the original music with no overt relation to the original lyrics; 4) translating the lyrics and adapting the music accordingly; 5) adapting the translation to the original music.

17 Tale modalità, come si è già accennato in precedenza, presenta limitate possibilità di applicazione. Inoltre, necessita di competenze specialistiche nella composizione musicale e nella strumentazione, pertanto esula dalla riflessione glottodidattica del presente lavoro e, di conseguenza, si ritiene di non approfondirlo ulteriormente in questa sede. 
Despite what some writers seem to think, song-texts are frequently translated for purposes other than singing. For example, music teachers and singers may need translations for study, for reading silently in recital programmes, or for reading aloud before the song is sung in the source language. (Low 2008, 1)

Nell'ambito dell'insegnamento delle lingue, la traduzione di una canzone straniera verso la lingua madre degli apprendenti rappresenta un'attività che spesso viene proposta come rinforzo alla comprensione testuale e può essere svolta anche in situazioni di autoapprendimento. Volendo esplorare, invece, modalità traduttive dalla lingua-madre dei discenti (nel presente caso l'italiano) verso la lingua straniera (qui il tedesco), ${ }^{18}$ possono essere prese in considerazione alcune traduzioni di canzoni italiane reperibili in rete, anche mettendo a confronto fra loro più versioni di una stessa canzone:

\begin{tabular}{lll}
\hline Adesso tu (Eros Ramazzotti 1986) & (Songtexte.com) & (Lyricstranslate.com) \\
\hline Nato ai bordi di periferia & $\begin{array}{l}\text { Geboren an den Rändern der } \\
\text { Peripherie, }\end{array}$ & Geboren am äußersten Stadtrand, \\
Dove i tram non vanno avanti più & $\begin{array}{l}\text { Wo die Straßenbahn nicht mehr } \\
\text { weiterfährt, }\end{array}$ & Wo die Straßenbahnen nicht mehr \\
Dove l'aria è popolare & Wo das Lied volkstümlich ist & weiterfahren, \\
È più facile sognare & Und es einfacher ist, zu träumen die Gegend dicht besiedelt ist, \\
Che guardare in faccia la realtà & Als der Realität ins Gesicht zu & Ist es einfacher, zu träumen, \\
& schauen. & Als der Wirklichkeit ins Auge zu \\
& & blicken. \\
E ci sei, adesso tu & Und jetzt bist Du da, & \\
A dare un senso ai giorni miei & Meinen Tagen einen Sinn zu geben & Un meinen Tagen einen Sinn zu \\
Va tutto bene dal momento che & Alles geht gut, ab dem Zeitpunkt, & Alles ist gut seit dem Moment, da \\
ci sei & an dem Du da bist & du \\
Adesso tu & Jetzt bist es Du & Jetzt hier bist. \\
Ma non dimentico & Aber ich vergesse nicht & Doch ich vergesse nicht \\
Tutti gli amici miei & Alle meine Freunde, & All meine Freunde, \\
Che sono ancora là & Die dort sind. & Die noch dort sind.
\end{tabular}

L'utilizzo di questo tipo di testi/traduzioni negli spazi didattici si può configurare come un'analisi contrastiva di testi paralleli, volta a promuovere l'ampliamento e il consolidamento delle competenze lessicali degli apprendenti. Partendo dalla presentazione di due o più Dienstübersetzungen del testo italiano e proponendo un esercizio di

18 Gli esempi sono scelti in base alle loro possibilità di utilizzo didattico in corsi di Lingua Tedesca e Traduzione a livello universitario. 
traduzione inversa (retro-traduzione), si evidenzieranno ad esempio: 1) parole ed espressioni in tedesco legate tra loro da un rapporto di sinonimia o semi-sinonimia (an den Rändern der Peripherie / am äußersten Stadtrand; der Realität ins Gesicht zu schauen / der Wirklichkeit ins Auge zu blicken; ab dem Zeitpunkt / seit dem Moment ecc.) dalle quali far scaturire una riflessione sulle diverse sfumature di significato in lingua straniera e/o su aspetti sociolinguistici legati a diversi registri e situazioni comunicative; 2) eventuali errori d'interpretazione da parte dei traduttori causati dalla polisemia di alcuni termini e dall'asimmetria delle due lingue coinvolte («dove l'aria è popolare»: wo das Lied volkstümlich ist / wo die Gegend dicht besiedelt ist). Tali osservazioni, accompagnate da esercizi atti a fissare e richiamare il lessico acquisito calandolo in concrete situazioni comunicative, aiuterà a sviluppare negli apprendenti una certa sensibilità e flessibilità nell'uso della lingua straniera.

Inoltre, l'analisi contrastiva delle diverse traduzioni potrà facilitare una riflessione sulle scelte traduttive in genere - con particolare riferimento alle scelte lessicali - e agevolare l'introduzione dei concetti di traduzione comunicativa e di equivalenza connotativa.

A livello di interpretazione testuale, infine, la canzone potrà essere messa a confronto con altre canzoni italiane e tedesche che affrontano lo stesso argomento, ${ }^{19}$ focalizzandosi su analogie e differenze tanto nelle modalità di sviluppo del tema quanto negli aspetti più strettamente legati alle due culture a confronto.

\subsection{Tradurre canzoni per la lettura}

Mentre una traduzione/parafrasi del tipo mostrato sopra non prevede vincoli se non quelli imposti dalla capacità del traduttore di comprendere il testo fonte e renderne in modo chiaro il senso nel testo di arrivo, la traduzione delle canzoni destinata alla lettura potrà essere, invece, affrontata con le stesse modalità della poetische Übersetzung..$^{20} \mathrm{Nel}$ caso di traduzione poetica, infatti, il traduttore sarà costretto a confrontarsi con problemi legati alla polisemia delle parole, agli artifici retorici, alle licenze poetiche, ai riferimenti culturali ecc. Si tratta, dunque, di una traduzione di tipo semantico, che però si applica di preferenza ai testi di quei cantautori ai quali è ri-

19 Nel caso di Adesso tu (Eros Ramazzotti 1986), il tema è quello della vita dei giovani nelle periferie urbane, argomento affrontato, per esempio, anche in Anna e Marco (Lucio Dalla 1979), in Vorstadt (AberHallo 2019) e in molte altre canzoni in entrambe le lingue.

20 A proposito della traduzione del testo poetico Wittbrodt $(1995,116-21)$ distingue fra cinque differenti approcci: 1) strukturtreue Übersetzung; 2) sinntreue Übersetzung; 3) wirkungstreue Übersetzung; 4) reimlose Übersetzung; 5) adaptierende Übersetzung. 
conosciuta anche fama di poeti e i cui versi vengono raccolti in volumi antologici oppure diffusi in rete attraverso siti tematici: ${ }^{21}$

\begin{tabular}{ll}
\hline $\begin{array}{l}\text { La guerra di Piero } \\
\text { (Fabrizio De André 1968) }\end{array}$ & $\begin{array}{l}\text { (Antiwarsongs.org - } \\
\text { trad. ted. di Christoph Gerhard) }\end{array}$ \\
\hline $\begin{array}{l}\text { Dormi sepolto in un campo di grano } \\
\text { Non è la rosa non è il tulipano }\end{array}$ & $\begin{array}{l}\text { Auf einem Kornfeld ruhst du in Frieden, } \\
\text { Es schmückt keine Rose dein einsames } \\
\text { Grab, }\end{array}$ \\
$\begin{array}{l}\text { Che ti fan veglia dall'ombra dei fossi } \\
\text { Es sind nur Tausende roter Mohnblumen, } \\
\text { Die dort erinnern an den, der da starb. }\end{array}$ \\
$\begin{array}{l}\text { Lungo le sponde del mio torrente } \\
\text { Voglio che scendano i lucci argentati } \\
\text { Non più i cadaveri dei soldati }\end{array}$ & $\begin{array}{l}\text { In diesem Flusse, an seinen Gestaden, } \\
\text { Jetzt stranden hier Leichen von den }\end{array}$ \\
Portati in braccio dalla corrente & Soldaten, \\
\hline
\end{tabular}

Trattandosi in questo caso di canzoni/poesie e relative traduzioni (poetiche), il loro utilizzo didattico non può prescindere da un approccio di tipo estetico che parta dall'analisi dell'originale per poi estendersi alla versione tradotta in lingua straniera. Qui, dunque, l'attenzione non potrà essere focalizzata solo su aspetti morfosintattici e/o di vocabolario, ma piuttosto sul legame che intercorre fra le scelte linguistiche e stilistiche e il loro effetto sui fruitori, agevolando un confronto con la complessità del tessuto testuale delle due lingue e, al tempo stesso, favorendo una lettura critica della canzone come poesia. ${ }^{22}$ Ad esempio, non sorprende che, nella ricerca di un effetto estetico uguale o perlomeno simile a quello del testo originale, nella traduzione si assista all'eliminazione di alcuni elementi (il tulipano, l'ombra dei fossi ecc.), all'inserimento di nuove informazioni, oppu-

21 Nel panorama musicale italiano si pensi a Fabrizio De André, Francesco De Gregori, Francesco Guccini o, a livello internazionale, a Bob Dylan, Leonard Cohen, Jim Morrison ecc. In particolare i testi delle canzoni di Fabrizio De André sono considerati da alcuni critici come vere e proprie poesie, tanto da essere inseriti, già dai primi anni Settanta, in varie antologie scolastiche di letteratura. In internet è possibile reperire versioni in varie lingue dei suoi maggiori successi, sia come traduzioni di servizio, sia come traduzioni poetiche.

22 A questo proposito Balboni $(2008,127)$ afferma che: «L'analisi di canzoni può essere utile [...] per introdurre alla comprensione della letterarietà, cioè di quei tratti linguistici, formali, che differenziano un testo con finalità estetiche, come le canzoni, da un testo quotidiano; non ci riferiamo qui solo a canzoni ormai entrate nelle antologie, da Pisci spada di Modugno ai testi di De André, ma anche a canzoni come quelle di Mogol e Battisti, che giocano sull'evocatività del lessico, o quelle di Vasco Rossi che sono dense di metafore e altre figure profondamente innovate, ma che, come in Sally, possono facilmente introdurre alla riflessione sul concetto di opera aperta e quindi sul ruolo dell'ascoltatore nel completare il testo, nel giungere a comprendere quel che non è detto». 
re che il torrente si trasformi in un Fluss e i lucci argentati diventino Forellen (evocando, tra l'altro, un famoso Lied di Franz Schubert) senza che il testo di arrivo subisca perdite in termini di significato.

Pertanto, l'introduzione di questo tipo di testi nella didattica universitaria potrà risultare utile per sensibilizzare gli apprendenti nei confronti del linguaggio poetico, tanto nella lingua madre quanto in quella straniera, offrendo loro strumenti di analisi e strategie di lettura efficaci per affrontare testi complessi, nonché far maturare in loro la coscienza del proprio sviluppo di comprensione e di approfondimento linguistico, investendo una pluralità di piani (linguistico, cognitivo, culturale).

\subsection{Tradurre canzoni per la performance canora}

Nei casi fin qui presentati, come si è detto, la canzone è vista come testo esclusivamente linguistico e la traduzione è improntata alle aspettative di un fruitore modello (lo studente che vuole comprendere il testo di una canzone, il lettore di poesie) in grado di riconoscerla come traduzione.

Molto diverso, invece, è il caso in cui una canzone venga tradotta per essere cantata in lingua straniera. In tale circostanza, infatti, essa va considerata nella propria natura di testo plurisemiotico e le scelte traduttive, come si è già accennato, saranno vincolate alle esigenze della musica e della performance..$^{23}$

In questo senso diviene fondamentale il concetto di singability, ritenuto da Low come il principale criterio con il quale un traduttore di testi destinati all'esecuzione canora deve confrontarsi in quanto:

Just as drama-translation requires words that can be performed as part of an integrated whole (which also includes gestures, costumes, lighting etc.), so a singable song-translation requires 'performability'. (Low 2005, 192)

Pertanto, il principio di singability - definita da Franzon $(2008,373)$ come «musico-verbal fit of a text to music» - sarà determinante nell'individuazione delle strategie traduttive da adottare per ricreare nel prototesto quel rapporto di corrispondenza fra musica e parole presente nel metatesto e funzionale alla performabilità della can-

23 A questo proposito Kaindl sottolinea che: «Popular music is in a dialogic relationship with various types of verbal, musical, visual as well as social and cultural elements. [...] Semiotics would permit a holistic analysis of the interaction and interdependence of the various elements of popular songs and could permit a deeper understanding of their role and influence in translation» (Kaindl 2005a, 259). 
zone anche in una lingua diversa dall'originale. ${ }^{24}$ In questo senso il concetto di singability si sostituisce allo skopos tradizionale della 'fedeltà traduttiva' nel senso che il grado di fedeltà al testo verbale dell'originale deve essere valutato in relazione alle esigenze del testo musicale e all'effetto artistico che si intende ricreare, senza prescindere dalle reali possibilità espressive delle diverse lingue di arrivo. ${ }^{25}$

In questo senso, guardando alle versioni straniere di canzoni italiane e considerando il principio della singability come scopo ultimo dell'atto traslatorio, ci si imbatterà più di sovente in traduzioni totali o parziali in riferimento alle lingue romanze, mentre per altre lingue di arrivo (germaniche, slave ecc.) saranno più frequenti le operazioni di riscrittura del testo con richiami più o meno evidenti all'originale: ${ }^{26}$

\begin{tabular}{|c|c|c|}
\hline $\begin{array}{l}\text { Piccolo uomo } \\
\text { (Mia Martini 1972) }\end{array}$ & $\begin{array}{l}\text { Pequeño hombre } \\
\text { (Mia Martini 1974) }\end{array}$ & $\begin{array}{l}\text { Tout petit homme } \\
\text { (Mia Martini 1974) }\end{array}$ \\
\hline Due mani fredde nelle tue & Dos manos frías sobre ti & $\begin{array}{l}\text { Mes mains reposent dans les } \\
\text { tiennes }\end{array}$ \\
\hline Bianche colombe dell'addio & Blancas palomas de un destino & $\begin{array}{l}\text { Comme des colombes qui prend } \\
\text { froid }\end{array}$ \\
\hline Che giorno triste questo mio & Que en este día triste y frío & Pourquoi se faire tant de peine \\
\hline Oggi tu ti liberi di me & Dicen que ya no me quieres más & Tout au fond de nous on sait déjà \\
\hline Di me che sono tanto fragile & A mí que soy tan frágil que jamás & $\begin{array}{l}\text { Que l'un sans l'autre on se perd } \\
\text { dans la vie }\end{array}$ \\
\hline E senza te mi perderò & podré vivir en soledad & $\begin{array}{l}\text { Que moi sans toi j'ai peur. Je t'en } \\
\text { prie }\end{array}$ \\
\hline Piccolo uomo, non mandarmi via & Piccolo uomo, No me dejes hoy & Tout petit homme ne me quitte pas \\
\hline Io, piccola donna, morirei & Yo niña perdida moriré & Moi petite femmej'en mourrais \\
\hline È l'ultima occasione per vivere & Es mi última batalla por ser feliz & $\begin{array}{l}\text { L'amour pour nous sera ce qu'il } \\
\text { sera }\end{array}$ \\
\hline
\end{tabular}

24 Franzon (2008, 389 ss.) a tal riguardo opera una distinzione fra prosodic match, poetic match e semantic-reflexive match. A proposito delle conseguenze sul piano traduttivo si veda Delli Castelli 2019, 13 ss.

25 Il primo ostacolo che un traduttore di testi destinati all'esecuzione canora deve affrontare è legato proprio alle lingue coinvolte nel processo traduttivo. Fra lingue diverse, infatti, esiste sempre un'asimmetria che può coinvolgere più piani. Ciò comporta per il traduttore una sfida di notevole portata qualora sia chiamato a riprodurre nella lingua di arrivo versi che ricalchino il prototesto tanto nell'aspetto formale quanto nel significato. Non tutte le lingue, però, sono parimenti asimmetriche: lingue fra loro affini, infatti, presentano di norma maggiori similitudini, mentre lingue appartenenti a gruppi diversi sono per loro natura più distanti.

26 A proposito della riscrittura creativa del testo canoro Kaindl precisa che: «Aufgrund der im Zuge der Übersetzung häufig zu beobachtenden massiven Veränderungen, der oft schwierigen Etikettierung von Ausgangs - und Zieltext sowie der zahlreichen Bedingungsfaktoren, die die Übersetzung beeinflussen, stellt die Übersetzung von Popularmusik eine große Herausforderung für die Wissenschaft dar» (Kaindl 2005b, 119). 
Barbara Delli Castelli Canzoni per l'insegnamento linguistico-culturale del tedesco e per la didattica della traduzione

\begin{tabular}{|c|c|c|}
\hline Vedrai che non la perderò, no & $\begin{array}{l}\text { No me hagas que la pierda, amor, } \\
\text { no }\end{array}$ & Mais ne nous séparons jamais non \\
\hline È l'ultima occasione per vivere & Es mi última batalla por ser feliz & $\begin{array}{l}\text { L'amour pour nous sera ce qu'il } \\
\text { sera }\end{array}$ \\
\hline Avrò sbagliato, sì lo so & No me hagas que la pierda, amor & J'ai fait des erreurs oui je sais \\
\hline Ma insieme a te ci riuscirò & No vayas a dejarme amor & mais tout changera tu verras \\
\hline
\end{tabular}

\begin{tabular}{|c|c|}
\hline Auf der Welt (Mia Martini 1973) & Im Namen der Liebe (Su Kramer 1974) \\
\hline Warum bin ich nur so allein? & Schlaflose Nächte, weiß und kalt \\
\hline Warum muss ich so einsam sein? & $\begin{array}{l}\text { Wann kommt die Sonne - kommt sie } \\
\text { bald? }\end{array}$ \\
\hline $\begin{array}{l}\text { Ich habe niemand', der mich liebt } \\
\text { Und der mir die Hoffnung wiedergibt, } \\
\text { Damit ich einmal wieder lachen kann. } \\
\text { D’rum frag ich mich immer wieder: }\end{array}$ & $\begin{array}{l}\text { Deine Gedanken haben Angst, } \\
\text { sagen mir was Du nicht sagen kannst } \\
\text { Ich werde auch nicht weinen, glaube mir } \\
\text { Ich weiß, dass ich Dich bald verlier' }\end{array}$ \\
\hline $\begin{array}{l}\text { Wo auf der Welt gibt es ein Herz für mich? } \\
\text { (Oho!) }\end{array}$ & $\begin{array}{l}\text { Im Namen der Liebe, treib kein Spiel mit } \\
\text { mir! }\end{array}$ \\
\hline Wer auf dieser Welt reicht mir die Hand & Das macht mich so einsam und so leer \\
\hline Und zeigt mir seine Liebe tagaus, tagein, & $\begin{array}{l}\text { Die Wahrheit hilft, dass ich Dich } \\
\text { vergessen kann, }\end{array}$ \\
\hline Bei Regen und bei Sonnenschein? & sonst kann ich es vielleicht nie mehr \\
\hline $\begin{array}{l}\text { Ja, dann wär ich auf der Welt nicht mehr } \\
\text { so allein. }\end{array}$ & $\begin{array}{l}\text { Nur die Wahrheit hilft, dass ich Dich } \\
\text { einmal vergessen kann, }\end{array}$ \\
\hline Das wär zu schön, um wahr zu sein. & sonst kann ich es vielleicht nie mehr. \\
\hline Das wär zu schön, um wahr zu sein. & $\begin{array}{l}\text { D'rum mach den Abschied nicht so } \\
\text { schwer }\end{array}$ \\
\hline
\end{tabular}

Nell'esempio sopra riportato si può notare che la versione spagnola e quella francese affrontano il tema dell'amore e dell'abbandono con le medesime modalità del testo fonte, presentando alcuni versi che sono traduzioni letterali dei corrispettivi versi italiani e adattandone altri alle esigenze della mascherina musicale. In entrambi i casi, dunque, si può parlare di traduzioni, anche se la versione spagnola risulta più aderente al prototesto (traduzione totale), mentre quella francese si propone piuttosto come un adattamento (traduzione parziale). Le versioni tedesche, invece, sono esempi di due diverse riscritture del brano con testi di argomento simile, ma non identico all'originale, composti ex novo per la stessa partitura musicale.

Da un punto di vista didattico una comparazione delle traduzioni tra loro e con il testo di partenza dovrebbe in primo luogo consentire agli studenti di riflettere sulle impalcature delle diverse lingue e sul tipo di asimmetrie esistenti tra loro e, in secondo luogo, di: 1) individuare il tipo di approccio adottato dai traduttori (traduzione totale, parziale o riscrittura); 2) determinare quali livelli strutturali 
del testo fonte siano stati privilegiati (e quindi riprodotti) in sede di traduzione; 3) verificare se le scelte adottate siano riconducibili alle limitazioni imposte dalla lingua di arrivo e dagli aspetti linguistici non trasferibili, oppure siano imputabili al fatto che il traduttore/ paroliere abbia privilegiato un determinato livello rispetto ad altri considerati meno significativi.

In questo senso, infatti, oltre all'asimmetria fra lingue riscontrabile sul piano fonologico, prosodico, lessicale, sintattico ecc., nonché sul piano culturale, inteso sia come significati culturospecifici sia come convenzioni poetiche (tutti aspetti di interesse tanto per la lezione di lingua/cultura, quanto per quella di letteratura), si possono individuare vari altri elementi in grado di influenzare il passaggio di un testo canoro dalla lingua originale a una diversa, come per esempio:

- la prevalenza dell'aspetto ritmico: se il punto di forza di una canzone viene individuato nel suo impianto melodico e ritmico, il traduttore/paroliere sarà chiamato a concentrarsi primariamente sulla struttura dei versi, sulle sequenze sillabiche, nonché sull'uso rigoroso di accenti, pause e rime. Il testo di arrivo si presenterà come una strukturtreue Übersetzung, un adattamento o riscrittura che tenderà soprattutto a riprodurre gli aspetti ritmico-musicali dell'originale:

\begin{tabular}{ll}
\hline $\begin{array}{l}\text { Il paradiso } \\
\text { (Patty Pravo 1969) }\end{array}$ & $\begin{array}{l}\text { Das Paradies auf dieser Welt } \\
\text { (Patty Pravo 1969) }\end{array}$ \\
\hline Il paradiso tu vivrai & Das Paradies auf dieser Welt \\
Se tu scopri quel che hai & Nur mit Dir allein \\
Non ti accorgi che & kann ich glücklich sein \\
lo amo già te & \\
& Ich seh' dich vor mir, \\
La vita è così, & das Wunder beginnt \\
Tu quando non hai & Wir schweben dahin \\
Vuoi avere di più & Wie Blätter im Wind \\
E dopo che hai & Wir tanzen davon \\
Ti accorgi che tu & Und merken es kaum \\
Fermarti non puoi & Es ist wie ein Traum \\
E vuoi quel che vuoi &
\end{tabular}

L'uso didattico di questo tipo di testi/traduzioni richiederà un'attenta analisi della struttura metrica in termini di segmentazione versale, lunghezza delle parole, numero di sillabe, posizione degli accenti tonici e delle pause, schema rimico ecc., senza trascurare l'eventuale ricorso ad apocopi, elisioni o allungamenti che permettono alle parole di 'accomodarsi' all'interno dello schema musicale. Pertanto, la riflessione si focalizzerà più sugli aspetti formali del testo verbale che sulla sua organicità semantica. In questo caso l'obiettivo sarà, 
dunque, quello di facilitare gli studenti a familiarizzare con le norme metriche, cioè a confrontarsi con il concetto di musicalità del testo. Solo in un secondo momento si potrà passare ad analizzare corrispondenze e divergenze semantiche e a valutare il peso di eventuali elementi culturospecifici fra i due testi a confronto.

- La prevalenza dell'effetto sonoro di una parola o di una frase: nel caso in cui il testo di partenza sia caratterizzato dal suono e/o dalla ripetizione di una frase, di una parola o di una porzione di parola, anche il metatesto dovrà essere costruito con le medesime modalità, presentando sequenze verbali dal suono uguale, o comunque simile, a quelle del testo originale. Il risultato sarà una wirkungstreue Übersetzung, una riscrittura tesa soprattutto a riprodurre gli effetti sonori delle parole:

\begin{tabular}{ll}
\hline $\begin{array}{l}\text { Viva la pappa col pomodoro } \\
\text { (Rita Pavone 1964) }\end{array}$ & $\begin{array}{l}\text { Ich frage meinen Papa } \\
\text { (Rita Pavone 1965) }\end{array}$ \\
\hline Viva la pappa pappa & Ich frage meinen Papa \\
Col po-po-po-po-po-po-pomodoro & $\begin{array}{l}\text { Denn Papa-pa-pa-pa-pa weiß bescheid } \\
\text { Viva la pappa pappa }\end{array}$ \\
Ich frage meinen Papa \\
Che è un ca-po-po-po-po-po-polavoro & Denn Papa-pa-pa-pa-pa ist alright \\
Viva la pa-pa-pa-pa-pa & Ich frage meinen Papa-pa \\
Col po-po-po-modor & Ich hab' es nie bereut \\
\hline
\end{tabular}

Nel caso presentato qui sopra, ad esempio, il testo italiano è strutturato intorno al suono della parola 'pappa' e alla ripetizione delle sillabe 'pa' e 'po'. Il traduttore/paroliere tedesco, allo scopo di riprodurre lo stesso effetto sonoro, crea un testo semanticamente distante dall'originale, costruendolo intorno alla parola 'Papa' (papà) e alla ripetizione della sillaba 'pa'.

In questo caso, dunque, anche la riflessione didattica sulla musicalità dovrà spostare il proprio focus dal testo (inteso come l'insieme dei versi) al suono di singole parole. Pertanto, oltre a un'analisi della struttura metrica e a una ricognizione delle rime, sarà utile servirsi, ad esempio, di esercizi di categorizzazione del lessico in base alla prima o all'ultima sillaba e/o di esercizi mirati all'individuazione di nuove parole attraverso la sostituzione di un suono (iniziale, interno o finale), seguiti da una riflessione sulle possibili collocazioni e/o su altri tipi di fraseologismi. Ciò, oltre a facilitare l'apprendimento e la memorizzazione di nuovi elementi lessicali e a offrire un prezioso strumento di controllo dell'organizzazione del significato, sarà utile per avviare gli studenti a un'attività di scrittura creativa (poetica) in lingua straniera partendo da uno spunto sonoro e indirizzando questo elemento verso la produzione di senso.

- La prevalenza del carattere poetico del testo: se il tratto distintivo di una canzone risiede nella poeticità del testo, il tra- 
duttore/paroliere dovrà focalizzare la propria attenzione principalmente sul contenuto semantico-verbale. In questo caso il metatesto sarà frutto di una sinngetreue Übersetzung, una traduzione (poetica) fedele al significato delle parole, ma altresì attenta alla riproduzione degli aspetti formali e strutturali del testo di partenza:

\begin{tabular}{|c|c|}
\hline Il pescatore (Fabrizio De André 1968) & Der Fischer (Bierfiedler 2002) \\
\hline All'ombra dell'ultimo sole & Noch im letzten Tagesschein \\
\hline S'era assopito un pescatore & Schlief am Strand der Fischer ein \\
\hline E aveva un solco lungo il viso & Trug ein Lächeln im Gesicht \\
\hline Come una specie di sorriso & Wie die Furche von 'nem Schnitt \\
\hline Venne alla spiaggia un assassino & Ein Mörder kam am Strand entlang \\
\hline Due occhi grandi da bambino & Seine Augen schauten bang \\
\hline Due occhi enormi di paura & Kinderaugen voller Feuer \\
\hline Eran gli specchi di un'avventura & Auf der Flucht nach Abenteuer \\
\hline E chiese al vecchio 'dammi il pane & Bat den Alten: ,Gib mir Brot \\
\hline Ho poco tempo e troppa fame' & Hab kaum Zeit und bin in Not ${ }^{r}$ \\
\hline E chiese al vecchio 'dammi il vino & Bat den Alten: ,Gib mir Wein \\
\hline Ho sete e sono un assassino' & Ich könnt heut dein Mörder sein‘ \\
\hline
\end{tabular}

In questo caso, dunque, nella traduzione saranno coinvolti simultaneamente tutti i livelli, dalla struttura metrica, al suono delle parole fino all'aspetto semantico-evocativo, restituendoci nella lingua di arrivo un testo cantabile che sia il più vicino possibile a quello originale e che, al tempo stesso, possa costruire un 'ponte emozionale' tra culture differenti.

Infine, parlando di canzoni effettivamente interpretate in lingua straniera dagli stessi cantanti italiani o 'date in prestito' ad artisti locali (in questo caso tedeschi o di lingua tedesca), risulterà interessante approfondire le modalità di ricezione del testo nel nuovo ambiente, focalizzandosi in particolare sul successo ottenuto dal singolo brano, sul genere (o sub-genere) musicale nel quale esso è stato inquadrato nella cultura di arrivo e, nel caso di cantanti italiani, sulla notorietà ottenuta nel nuovo ambiente anche grazie all'interpretazione di altri brani. ${ }^{27}$

27 Un esempio emblematico in questo senso è quello offerto da Rita Pavone, ritenuta una delle interpreti più significative tanto della musica leggera italiana quanto della Schlager-Musik tedesca degli anni Sessanta. Oltre a versioni in tedesco di suoi brani italiani (Se fossi un uomo / Wenn ich ein Junge wär, 1963; Viva la pappa col pomodoro / Ich frage meinen Papa, 1965; Zucchero / Liebe könnte wie Zucker sein, 1969), Rita Pa- 
In base a quanto detto, dunque, il ricorso didattico a testi/traduzioni destinati alla performance canora può considerarsi come una sfida tanto complessa quanto affascinante, atta a favorire la riflessione sul rapporto semiotico fra musica e parole e su ciò che esso comporta in fase di trasferimento da un sistema linguistico-culturale ad un altro, tenendo conto delle peculiarità delle lingue coinvolte nel processo traduttivo, delle caratteristiche proprie del testo da trasferire e del grado di ricettività dell'ambiente di arrivo. Pertanto, l'analisi di tali testi/traduzioni permetterà di collocare il concetto stesso di traduzione in un contesto più ampio e meno prescrittivo, con pratiche variamente graduate che vanno dalla traduzione, appunto, all'adattamento, fino alla totale rielaborazione del testo, chiamando in gioco competenze linguistiche, traduttive e culturali.

\section{Conclusioni}

La musica leggera e le canzoni a essa riconducibili sono prodotti artistici di massa fra i più fruibili che possono essere culturospecifici e, al tempo stesso, trasversali a più culture. Le loro caratteristiche linguistico-formali (lessico, registri linguistici, modalità discorsive ecc.), nonché le peculiarità strutturali (versi organizzati in strofe e ritornelli, norme metriche, ripetitività di alcune porzioni di testo ecc.) e tematiche (temi universali o culturalmente connotati) ne fanno dei testi autentici, che ben si adattano a svariate esigenze didattiche, tanto nell'educazione linguistica quanto in quella letteraria. L'utilizzo delle canzoni, inoltre, può trovare ampio spazio anche nella didattica della traduzione, offrendo l'opportunità di lavorare con testi plurisemiotici per i quali la compresenza del codice verbale con quello musicale contribuisce a condurre la traduzione ai limiti delle sue stesse possibilità. Nel caso delle canzoni, infatti, il significato delle parole è solo una delle componenti che entrano in gioco nel processo di trasposizione e il suo peso andrà valutato in base ai diversi skopoi traduttivi, alle asimmetrie esistenti fra le lingue coinvolte, nonché alle caratteristiche artistico-estetiche del testo stesso. Pertanto, il passaggio di una canzone da una lingua/cultura a un'altra richiederà competenze linguistico-culturali che vanno al di là della sola abilità traduttiva, coinvolgendo anche la sfera della creatività e la capacità di sperimentazione in lingua straniera.

vone ha inciso anche brani esclusivamente per il mercato tedesco: Bye bye Blue Jeans (1964), Kiddy, Kiddy kiss me (1964), Arrivederci Hans (1968). Inoltre, alcuni suoi successi, negli anni, sono stati riproposti da altri cantanti come Nina Hagen (Wenn ich ein Junge wär, 1979) e Florian Haidt (Kiddy, Kiddy kiss me, 1983). 


\section{Bibliografia}

Badstübner-Kizik, C. (2010). «Kunst und Musik im Deutsch als Fremd- und Zweitsprache-Unterricht». Krumm, H.-J. et al. (Hrsgg), Deutsch als Fremdund Zweitsprache. Bd. 2. Berlin; New York: Walter de Gruyter, 1596-601. Handbücher zur Sprach- und Kommunikationswissenschaft 35/2.

Balboni, P.E. (2008). Fare educazione linguistica. Attività didattiche per Italiano L1 e L2, lingue straniere e lingue classiche. Torino: UTET.

Balboni, P.E. (2013). «Cultura e civiltà. Processi e prodotti». Baldi, B.; Borello, E.; Luise, M.C. (a cura di), Aspetti comunicativi e interculturali nell'insegnamento delle lingue. Cittadini europei dal nido all'università = Atti del Convegno $(\mathrm{Fi}-$ renze, 14 marzo 2013). Alessandria: Edizioni dell'Orso, 61-70.

Balboni, P.E. (2018). Sillabo di riferimento per l'insegnamento dell'italiano della musica. Venezia: Edizioni Ca' Foscari. http://dx.doi.org/10.30687/97888-6969-270-3. SAIL Studi sull'apprendimento e l'insegnamento linguistico 14

Bandini, F. (1996). «Una lingua poetica di consumo». Coveri, L. (a cura di), Parole in musica. Lingua e poesia nella canzone d'autore italiana. Novara: Interlinea, 27-35.

Bettermann, R. (2013). «Populäre Lieder im Deutschunterricht». Magali, M.; Chen, E.V. (Hrsgg), Kulturdidaktik im Unterricht Deutsch als Fremdsprache. Facetten der Vermittlung von Kultur und Landeskunde. Rio de Janeiro: APA, 103-21. https://bit.ly/2CFOoRG.

Blell, G.; Kupetz, R. (Hrsgg) (2010). Der Einsatz von Musik und die Entwicklung von audio literacy im Fremdsprachenunterricht. Frankfurt, Main u.a.: Peter Lang.

Bredella, L. (2002). Literarisches und interkulturelles Verstehen. Tübingen: Gunter Narr. Giessener Beiträge zur Fremdsprachendidaktik.

Caon, F. (2005). Un approccio umanistico affettivo all'insegnamento dell'italiano a non nativi. Venezia: Cafoscarina.

Caon, F.; Lobasso, F. (2008). «L'utilizzo della canzone per la promozione e l'insegnamento della lingua, della cultura e della letteratura italiana all'estero». Studi di glottodidattica, 2(1), 54-69. https://ojs.cimedoc.uniba. it/index.php/glottodidattica/article/view/218.

Caon, F.; Spaliviero, C. (2015). Educazione letteraria, linguistica, interculturale: intersezioni. Torino: Bonacci.

Colombo, A. (2005). «I valori, la lingua, l'immaginario. Trent'anni di dibattito sull'educazione letteraria». Lavinio, C. (a cura di), Educazione linguistica e educazione letteraria. Intersezioni e interazioni. Milano: Franco Angeli, 51-63.

Coveri, L. (2011). «Le canzoni che hanno fatto l'italiano». Benucci, E.; Setti, R. (a cura di), Italia linguistica. Gli ultimi 150 anni. Nuovi soggetti, nuove voci, un nuovo immaginario. Firenze: Accademia della Crusca; Le Lettere, 69-126.

Delli Castelli, B. (2019). «Canzoni italiane nella lezione di tedesco». Lend. Lingue e nuova didattica, 48(1), 8-20.

De Mauro, T. (1996). «Note sulla lingua dei cantautori dopo la rivoluzione degli anni '60». Coveri, L. (a cura di), Parole in musica. Lingua e poesia nella canzone d'autore italiana. Novara: Interlinea, 37-44.

Di Cristofaro Longo, G. (1993). Identità e cultura. Per un'antropologia della reciprocità. Roma: Studium.

Erdmenger M.; Istel, H.-W. (1973). Didaktik der Landeskunde. Ismaning: Max Hueber. 
Fabbri, F. (2001). «La canzone». Nattiez, J.-J. (a cura di), Enciclopedia della Musica. Il Novecento, vol. 1. Torino: Einaudi, 551-76.

Fabbri F. (2015). «Sui nomi delle musiche». Bonomi, I.; Coletti, V. (a cura di), L'italiano della musica nel mondo. Firenze: Accademia della Crusca; goWare, 168-77.

Franzon, J. (2008). «Choices in Song Translation. Singability in Print, Subtitles and Sung Performance». The Translator, 14(2), 373-99.

Gallo, P.; Reeg, U.; Simon, U. (Hrsgg) (2016). Sehen und Entdecken. Visuelle Darstellungen im DaF-Unterricht. Münster; New York u. a.: Waxmann.

Garzone, G.E. (2012). «Traduzione, riscrittura e manipolazione ideologica. Le 'cover' delle canzoni di Dylan negli anni '60». Altre modernità. Numero speciale: traduzione e riscrittura, 16-38. https://riviste.unimi.it/index. php/AMonline/article/view/2454/2678.

Gorlée, D.L. (ed.) (2005). Song and Significance. Virtues and Vices of Vocal Translation. Amsterdam; New York: Rodopi.

Hecke, C.; Surkamp, C. (Hrsgg) (2010). Bilder im Fremdsprachenunterricht. Neue Ansätze, Kompetenzen und Methoden. Tübingen: Gunter Narr. Giessener Beiträge zur Fremdsprachendidaktik.

Herzog, W. (2003). «Im Nebel des Ungefähren: Wenig Plausibilität für eine neue Kompetenz». Erwägen, Wissen, Ethik, 14(1), 178-80.

Kaindl, K. (2005a). «The Plurisemiotics of Pop Song Translation. Words, Music, Voice and Image». Gorlée 2005, 235-62.

Kaindl, K. (2005b). «Kreativität in der Übersetzung von Popularmusik». Lebende Sprachen, 50(3), 119-24.

Lavinio, C. (a cura di) (2005). Educazione linguistica e educazione letteraria. Intersezioni e interazioni. Milano: Franco Angeli.

Low, P. (2005). «The Pentathlon Approach to Translating Songs». Gorlée 2005, $185-212$.

Low, P. (2008). «Translating Songs that Rhyme». Perspectives. Studies in Translation Theory and Practice, 16(1-2), 1-20.

Luperini, R. (2013). Insegnare la letteratura oggi. Quinta edizione ampliata. Lecce: Manni.

Mayoral, R.; Kelly, D.; Gallardo, N. (1988). «Concept of Constrained Translation. Non-Linguistic Perspectives of Translation». Meta - Journal des trad ducteurs, 33(3), 356-67.

Martino, P. (2004). «La forma canzone tra teoria del discorso e della traduzione». Culture, 18 (2004), 191-206. http://www.club.it/culture/ culture2004/11culture2004.pdf.

Mecheril, P. (2003). «Behauptete Normalität: Vereinfachung als Modus der Thematisierung von Interkulturalität». Erwägen, Wissen, Ethik, 14(1), 198-201.

Melde, W. (1987). Zur Integration von Landeskunde und Kommunikation im Fremdsprachenunterricht. Tübingen: Gunter Narr. Tübinger Beiträge zur Linguistik 301.

Middleton, R. (1990). Studying Popular Music. Milton Keynes; Philadelphia: Open University Press.

Nida, E.A. (1964). Toward a Science of Translating: With Special Reference to Principles and Procedures Involved in Bible Translating. Leiden: Brill.

Snaidero, T. (2008). «L'insegnamento della cultura nella didattica delle lingue straniere in Germania». Studi di Glottodidattica, 2(1), 120-32. https:// ojs.cimedoc.uniba.it/index.php/glottodidattica/article/ view/222. 
Spaliviero, C. (2015). «Didattica della letteratura italiana attraverso le canzoni». Bollettino Itals. Supplemento EL.LE, 13(61), 8-25. https://bit. ly/390JvNg.

Surugiu, O. (2010). «From Music on the Stage to Music on the Page. Song Translation in Language Teaching». Luminiţa, A.-C.; Chiper, S.; Sandulovici, A. (eds), Language, Culture and Change, Higher Education between Tradition and Innovation, vol. 2. Iaşi: Editura Universităţii «Alexandru loan Cuza», 117-24.

Thürmann, E. (1994). «Fremdsprachenunterricht, Landeskunde und interkulturelle Erziehung». Die neueren Sprachen, 93(4), 316-34.

Vermeer, H.J. (1996). A Skopos Theory of Translation (some Arguments for and Against). Heidelberg: TEXTconTEXT.

Welke, T.; Faistauer, R. (Hrsgg) (2015). Film im DaF/DaZ-Unterricht. Wien: Praesens. Beiträge der 15. IDT Bozen.

Wittbrodt, A. (1995). Verfahren der Gedichtübersetzung. Definition, Klassifikation, Charakterisierung. Frankfurt am Main: Peter Lang.

Zanettin, F. (1998). «Fumetti e traduzione multimediale. Tra codice verbale e codice visivo». inTRAlinea, vol. 1. http://www.intralinea.org/archive/article/1622.

\section{Siti internet consultati}

Canzoni contro la guerra. https://www. antiwarsongs.org. Lyricstranslate. https://lyricstranslate.com. Songtexte. https://www.songtexte.com. 



\title{
EL.LE
}

Vol. 9 - Num. 1 - Marzo 2020

\section{Variabilità fonetico-sintattica nella Commercial Contemporary Music come oggetto di studio per le classi avanzate di inglese come L2}

\author{
Gianmarco Perna \\ Università di Roma La Sapienza, Italia
}

\begin{abstract}
The aim of this research is to set up a consistent theorical arrangement concerning the socio-linguistic and socio-phonetic aspects of contemporary commercial music (CCM), which appears to be more and more uneven and manifold, also taking into account the previous research by Trudgill (1983), Simpson (1999) and others. The massive use of phonetic variation and style switching in contemporary Western singing style is here analyzed mainly focussing on its phonetic, cognitive and semantic relevance. Some examples of metric, socio-linguistic and stylistic variation are provided afterwards, so that a global diagram of linguistic habits might be traced. Other collateral aspects are recollected, mostly concerning the effects of CCM experience on L2 users.
\end{abstract}

Keywords Sociolinguistics. Phonetics. Style switching. Popular music. Commercial contemporary music.

Sommario 1 Introduzione. -2 Metodo. - 3 Variabilità lessico-sintattica e possibili rischi. - 4 Variabilità fonetica. - 4.1 Variazione a fine articolatorio-performativo. - 4.2 Variazione a fine metrico. - 4.3 Variazione a fine stilistico. - 4.4 Variazione di carattere idiolettico e sociolinguistico. - 4.5 Esempi di variazione geografico-dialettale. - 5 Conclusioni.

\section{Peer review}

Submitted 2019-10-24

Accepted 2019-11-02

Published 2020-07-30

\section{Open access}

(c) 2020 | ()(1) Creative Commons Attribution 4.0 International Public License 


\section{Introduzione}

È possibile introdurre positivamente lo studio della produzione commerciale contemporanea nel discorso della linguistica e come sua applicazione pratica? A ben vedere, la Commercial Contemporary Music (CCM) si adatta facilmente a diverse inchieste della linguistica contemporanea, soprattutto quelle che riguardano l'utilizzo della musica nell'ambito educativo. Diversi linguisti (Dubin 1975; Schwartz 2013; Richards 1969; Israeli 2013; Nishina 2016, per citarne alcuni) hanno analizzato i contesti e le possibilità di insegnare tramite canzoni la lingua inglese come L2, vista la loro efficienza nel migliorare la consapevolezza linguistica dello studente in ogni aspetto formativo ( $v o$ cabulary, comprehension, listening, speaking e writing) come suggeriscono Boothe e West (2015).

Se gran parte degli studi proposti, pur indirizzati a studenti di ogni età, sembrano particolarmente adatti nelle classi di apprendimento inferiori e superiori (potrebbero essere destinati agli studenti italiani di primo e secondo grado) poiché incentrati sulla capacità imitativa dello studente di fronte alle soluzioni standardizzate presenti nelle canzoni e sull'implementazione incidentale delle routine linguistiche insite in esse, gli ambiti avanzati di studio e ricerca sembrano ancora parzialmente estranei a tale argomento. La musica commerciale in lingua inglese sembra però adatta anche a quelle situazioni didattiche e accademiche fortemente sperimentali e può trovare originali spazi di applicazione finora rimasti impensati rispetto a quanto i più recenti studi di linguistica in tutti i suoi aspetti (studi di fonetica e fonologia, sociolinguistica e stilistica, in maggioranza) hanno finora offerto, alla stregua delle innovative proposte educative riguardanti i prodotti audiovisivi (Ranzato 2016, 2019) sempre più presenti nelle classi universitarie italiane e globali.

In primo luogo, il testo di una canzone è inserito, senza dubbio, nel discorso della fonetica applicata, sia per ciò che concerne l'analisi qualitativa e quantitativa dello 'strumento voce', che per quella della ricezione auricolare del testo foneticamente espresso. Da questo punto di vista, la ricerca proposta coinvolge anche la fonetica comparativa e la socio-fonetica, con lo scopo principale di superare la 'soluzione americanizzante' individuata da Trudgill (1983) in seno al Brit Pop beatlesiano: piuttosto che individuare un'unica formula che risolva le più dispari rappresentazioni linguistiche, è maggiormente auspicabile arrendersi al fatto che, offrendo la musica commerciale di fatto diverse e disomogenee realizzazioni fonetiche, ad esse vadano a contribuire in cooperazione numerosi fattori.

In una ricerca con quest'obiettivo si rende necessario, innanzitutto, ridurre la natura linguistica della produzione musicale contemporanea al concetto singolo di variabilità: come in un fan deck di innumerevoli sfumature di un singolo colore, un solo elemento fonetico 
presente in una canzone in lingua inglese (la qualità della produzione fonica del fonema - $\alpha$ nella parola mama secondo la pronuncia standard americana, ${ }^{1}$ ad esempio) può presentarsi in espressioni numerose e internamente variabili (può coincidere con -æ o con -ə, ad esempio, nel caso di un artista britannico ${ }^{2}$ ). Muovendoci da un'artista ad un altro, o da una singola traccia ad un'altra, lo stesso elemento può variare, come può presentarsi in vesti diversi a seconda del genere musicale, della lunghezza dell'emissione di quel tratto analizzata, e così via. Ne recepiamo non di meno un possibile pericolo didattico, in quanto lo studente delle classi inferiori (a cui viene fatta solamente ascoltare o assegnata per casa una canzone) che sia privo di nozioni linguistiche avanzate, potrebbe essere indotto da una singola canzone a far coincidere la pronuncia di due fonemi, o a utilizzare la forma non-standard ain't per la negazione delle forme to be o to have, mentre gli stessi elementi in variazione sono estremamente utili per lo studente di sociolinguistica delle classi avanzate, poiché testimonianze di valore sociolinguistico, afferenti alla fonetica regionale o dovute al contesto sociale e culturale di provenienza o di destinazione della canzone stessa. Obiettivo della seguente ricerca è armonizzare i diversi aspetti di studio proposti, analizzando in maniera contrastiva la variabilità sintattica e fonetica interna alla CCM nella sua divergenza dallo Standard English (con received pronunciation come base didattico-normativa), per individuare una possibilità teorica di categorizzazione dell'intero e ricco corpus culturale che la collezione dei frammenti musicali del mondo presente può costituire per la società a venire.

\section{Metodo}

Limitandoci allo studio della canzone registrata (ulteriori studi potrebbero interessare le variazioni fonetiche che intercorrono tra la traccia registrata e l'esibizione dal vivo), e ricollegandoci a McGregor (2009), in cui si riconosce che «absolutely free variation is rare. Usually there are reasons for it, perhaps the speaker's dialect, perhaps the emphasis the speaker wants to put on the word» (55), saranno oggetto di discussione tracce registrate e pubblicate tra il $1978 \mathrm{e}$ il 2018. Il corpus di riferimento sarà un totale di 48 tracce registrate e diffuse tra il 1978 e il 2018, realizzate in maggioranza da americani $(66,6 \%)$, poi britannici $(27,1 \%)$, australiani $(4,2 \%)$ e infine inglesi L2 (2,1\%). Tra gli artisti americani inseriremo anche i performer di

2 https://www.macmillandictionary.com/pronunciation/british/mama. 
origine afroamericana, sia per una questione geografica, sia in quanto spesso le dinamiche fonetiche che sono evidenti nel loro speech vengono minimizzate o del tutto annullate nel canto. All'interno della categoria 'americani', in ogni caso, gli artisti espressamente AAVE (Afro-American Vernacular English) rappresentano il 37\% circa.

Distribuiremo la nostra speculazione secondo varie argomentazioni con un intento dimostrativo-esemplificativo: saranno innanzitutto individuati i macro-processi sintattici e fonetici, con i necessari raffronti teorici; quanto esposto sarà a seguire raffrontato con casi particolari in cui essi sono evidenziabili.

Individuiamo come target di riferimento delle soluzioni proposte gli studenti delle classi inferiori e superiori, per ciò che concerne la variazione sintattico-semantica, e studenti d'inglese come L2 in situazioni accademiche e/o avanzate, in merito alle variazioni fonetiche che necessitano di un consolidato sostegno teorico affinché siano coerentemente apprezzate. Riconosciamo che il lavoro che andiamo ad affrontare è un lavoro in itinere, poiché inerente ad una frazione culturale raramente affrontata dalla linguistica teorica o pragmatica (positivamente inclusa, invece, nella letteratura sociale e culturale, e nello studio della storia della musica: si veda Middleton 1990), ed è ricco di sfide in quanto si propone di evidenziare cambiamenti in atto nella cultura del nostro tempo, suggerire piste di ricerca sociolinguistica che possano aiutare gli studenti a riconoscere i caratteri teorici della comunicazione in lingua inglese nella loro applicazione commerciale al discorso musicale, arricchendo il mero studio della grammatica con una teoria sociolinguistica di background e introducendo nuovi elementi di analisi della CCM, quali la necessità di integrare fattori metrico-stilistici, fonetico-articolatori, nonché geografico-dialettali alla base delle idiosincrasie fonetiche (Trudgill 2000) riscontrabili nel canto commerciale contemporaneo.

\section{Variabilità lessico-sintattica e possibili rischi}

Come recentemente suggerito da Eiter (2017), la ricchezza della musica commerciale contemporanea in lingua inglese si esprime anche nella sua variabilità lessico-sintattica. Nel suo lavoro l'autore individua elementi di variazione non-standard ampiamente riscontrabili nel pop: sostituzione di doesn't con don't, in linea con la teoria sociolinguistica dell'inglese vernacolare di Trudgill (2009); la presenza di doppia negazione e ridondanze nelle costruzioni negative; l'uso dominante e multiforme di ain't, espressione sia del vernacolare afroamericano (Howe 2005) che dell'inglese orale informale. Lo studio analitico e comparativo del vocabolario e dei sistemi sintattici riscontrabili in quell'inglese 'astratto' che appartiene alla produzione musicale 
popolare contemporanea permette di qualificare il linguaggio d'uso e di riscontrarne variazioni rispetto al sistema linguistico quotidiano, analisi che si adattano ad essere generalmente implementate nei processi di second language acquisition in numerosi approcci didattici e per studenti di età differenti. Partendo dall'analisi induttiva di Eiter (2017, 20-7, 29-31), andiamo ad ampliare le forme di variazione lessico-sintattica in seno alla produzione commerciale contemporanea. La tabella 1 racchiude alcuni esempi delle abitudini succitate.

Tabella 1 Variazioni lessico-sintattiche riscontrabili nella CCM, suddivise per categoria

\begin{tabular}{|c|c|c|}
\hline \multirow[t]{7}{*}{ Riduzione della terza persona } & Nicki Minaj - Anaconda (2014) AAVE & $\begin{array}{l}{[\ldots] \text { My anaconda don't want none }} \\
\text { unless you got buns, hun }[\ldots]\end{array}$ \\
\hline & $\begin{array}{l}\text { Kesha - TiK Tok (2009) } \\
\text { GA }\end{array}$ & $\begin{array}{l}{[\ldots] \text { Tick-tock on the block, but the }} \\
\text { party don't stop [...] }\end{array}$ \\
\hline & $\begin{array}{l}\text { Jay-Z ft. Pharrel Williams- Excuse } \\
\text { me miss (2002) } \\
\text { AAVE }\end{array}$ & $\begin{array}{l}\text { [...] He don't wife 'em, he one } \\
\text { nights 'em!/Now she don't like } \\
\text { him [...] }\end{array}$ \\
\hline & $\begin{array}{l}\text { Genesis - Invisible Touch (1986) } \\
\text { Cockney }\end{array}$ & $\begin{array}{l}{[\ldots] \text { She don't like losing, to her it's }} \\
\text { still a game }[\ldots]\end{array}$ \\
\hline & $\begin{array}{l}\text { Shaggy ft. Ricardo Ducent- } I t \\
\text { Wasn't Me (2008) } \\
\text { AAVE }\end{array}$ & $\begin{array}{l}{[\ldots . .] \text { If she say a night, convince her }} \\
\text { say a day }[\ldots]\end{array}$ \\
\hline & $\begin{array}{l}\text { Ty Dolla Sign ft. B.o.B - Paranoid } \\
\text { (2014) } \\
\text { AAVE }\end{array}$ & $\begin{array}{l}{[\ldots] \text { She run her mouth so much }} \\
\text { she can't hear her own thoughts } \\
{[\ldots]}\end{array}$ \\
\hline & $\begin{array}{l}\text { Iggy Azalea ft. Jennifer } \\
\text { Hudson - Trouble (2014) } \\
\text { Australian Accent (cfr. Par.II) }\end{array}$ & $\begin{array}{l}{[\ldots] \text { See what he doin' to me make }} \\
\text { have to shout it out }[\ldots]\end{array}$ \\
\hline \multirow[t]{6}{*}{ Uso di ain’t } & $\begin{array}{l}\text { Taylor Swift - Delicate (2017) } \\
\text { GA }\end{array}$ & $\begin{array}{l}{[\ldots] \text { This ain't for the best, my }} \\
\text { reputation's never been worse, } \\
\text { so }[\ldots]\end{array}$ \\
\hline & $\begin{array}{l}\text { Train - Hey, Soul Sister (2009) } \\
\text { GA }\end{array}$ & $\begin{array}{l}{[\ldots] \text { Hey, soul sister, ain't that Mr. }} \\
\text { Mister on the radio, stereo? [...] }\end{array}$ \\
\hline & $\begin{array}{l}\text { Selena Gomez ft. Kygo - It Ain't Me } \\
(2017) \\
\text { GA }\end{array}$ & $\begin{array}{l}\text { [...] Who's gonna walk you through } \\
\text { the dark side of the morning? } \\
\text { It ain't me [...] }\end{array}$ \\
\hline & $\begin{array}{l}\text { Paula Abdul - The Way That You } \\
\text { Love Me (1988) } \\
\text { GA }\end{array}$ & $\begin{array}{l}{[\ldots] \text { It ain't the clothes that you }} \\
\text { wear / It ain't the things that you } \\
\text { buy [...] }\end{array}$ \\
\hline & $\begin{array}{l}\text { Alicia Keys - If I Ain’t Got You (2003) } \\
\text { AAVE }\end{array}$ & $\begin{array}{l}\text { [...] But everything means nothing } \\
\text { / If I ain't got you, yeah [...] }\end{array}$ \\
\hline & $\begin{array}{l}50 \text { Cent - Candy Shop (2005) } \\
\text { AAVE }\end{array}$ & $\begin{array}{l}{[\ldots] \text { You ain't never heard a sound }} \\
\text { like this before/ 'Cause I ain't never } \\
\text { put it down like this [...] }\end{array}$ \\
\hline
\end{tabular}




\begin{tabular}{|c|c|c|}
\hline $\begin{array}{l}\text { Ridondanza nelle costruzioni } \\
\text { negative }\end{array}$ & $\begin{array}{l}\text { Ariana Grande - No Tears Left to Cry } \\
\text { (2018) } \\
\text { GA }\end{array}$ & $\begin{array}{l}{[. . .] \text { Ain't got no tears left to cry, so }} \\
\text { I'm picking it up }[\ldots]\end{array}$ \\
\hline & $\begin{array}{l}\text { Lady Gaga - Born This Way (2011) } \\
\text { GA }\end{array}$ & $\begin{array}{l}{[\ldots] \text { Oh there ain't no other way, }} \\
\text { baby I was born this way [...] }\end{array}$ \\
\hline & $\begin{array}{l}\text { Simply Red - If You Don't Know Me } \\
\text { by Now (1989) } \\
\text { Manchester Accent }\end{array}$ & $\begin{array}{l}{[\ldots] \text { I ain't gonna do nothing to }} \\
\text { break up our happy home }[\ldots]\end{array}$ \\
\hline & $\begin{array}{l}\text { The Rolling Stones - (I Can't Get no) } \\
\text { Satisfaction (1965) } \\
\text { Cockney }\end{array}$ & $\begin{array}{l}{[\ldots] \text { I can't get no satisfaction / }} \\
\text { 'Cause I try and I try and I try and } \\
\text { I try [...] }\end{array}$ \\
\hline & $\begin{array}{l}\text { TLC - No Scrubs (1992) } \\
\text { AAVE }\end{array}$ & $\begin{array}{l}{[\ldots] \text { I don't want none of your time }} \\
\text { and, no/ I don't want no scrub [...] }\end{array}$ \\
\hline & $\begin{array}{l}\text { Gwen Stefani - Hollaback Girl } \\
\text { (2004) } \\
\text { GA }\end{array}$ & $\begin{array}{l}{[\ldots] \text { 'Cause I ain't no hollaback girl }} \\
\text { I ain't no hollaback girl [...] }\end{array}$ \\
\hline & $\begin{array}{l}\text { Haddaway - What Is Love (1993) } \\
\text { GA }\end{array}$ & $\begin{array}{l}{[\ldots] \text { Baby don't hurt me/Don't hurt }} \\
\text { me/ No more }[. . .]\end{array}$ \\
\hline & $\begin{array}{l}\text { Destiny's Child - Bills Bills Bills } \\
\text { (1999) } \\
\text { GA }\end{array}$ & $\begin{array}{l}{[\ldots] \text { Don't know where none of }} \\
\text { these calls come from }[\ldots]\end{array}$ \\
\hline & $\begin{array}{l}\text { Rihanna - Don't Stop the Music } \\
\text { (2007) } \\
\text { AAVE }\end{array}$ & $\begin{array}{l}{[\ldots] \text { I wasn't looking for nobody }} \\
\text { when you looked my way }[\ldots]\end{array}$ \\
\hline
\end{tabular}

Vi è, del resto, la possibilità che il testo di una canzone possa avere effetti inaspettati sulla platea di $\operatorname{arrivo}^{3}$ e che sia suscettibile di errata o mancata comprensione da parte del pubblico. Nel caso di Die Young di Ke\$ha (Warrior, 2012), la coincidenza temporale tra la diffusione radiofonica della traccia e la strage nella scuola americana di New Town ha portato Billboard ad escluderla dal palinsesto musicale. ${ }^{4}$ In If I Were a Boy di Beyoncé Knowles (I am...Sasha Fierce, 2008), scritta da Toby Gad e BC Jean, l'uso di una prospettiva ipotetica permette una definizione comportamentale di 'uomo' che, pur carica di femminismo, potrebbe non coincidere con la totalità della porzione di tessuto sociale di riferimento. Allo stesso modo, l'uso di terminologia sociale minoritaria in Born This Way di Lady Gaga (Born This Way, 2011), come orient e chola, nonché il gioco di parole ([...] don't be a drag, just be a queen) sembra far sfumare, infine, l'intenzione rivoluzionaria dell'artista (Williams 2014). All'insegnante che voglia utilizzare una canzone come oggetto didattico, suggeriamo di tenere a mente le diverse possibili risposte degli studen-

3 In merito all'uso di linguaggio violento nei testi musicali si veda Anderson 2003.

4 https://www.theguardian.com/music/2012/dec/19/kesha-die-young-us-radio. 
ti a stimoli d'indagine di questa natura, provando ad analizzarne il testo anche per come l'apparato semantico e sociale che essa evoca possa esser recepito. ${ }^{5}$

\section{Variabilità fonetica}

Trudgill (1983) individua sei caratteristiche del Brit Pop anni Cinquanta che avvicinano la pronuncia dei performer inglesi a quella del General American, da qui indicato solamente come GA. Giustificato come espediente per la diffusione anche nel mercato americano, e da qui globale, tale argomento, che Trudgill (1983) ha modernamente introdotto negli studi di sociolinguistica, è stato successivamente ripreso e ampliato da altri linguisti. Simpson $(1999,345)$ ha proposto un modello di cinque fattori fonetici americani che contribuiscono alla costruzione del cosiddetto «ibrido transatlantico» che accomuna molti artisti di lingua inglese, e che riguarda anche chi adopera l'inglese come L2, come individua Asgautsen (2017).

Analizzando i testi e la pronuncia del frontman degli Arctic Monkeys, Alex Turner, Beal $(2009,229)$ opera un confronto con le teorie dei due linguisti precedenti, constatando quanto segue: «within the language-ideological framework, linguistic features are seen to become associated with social values so that they acquire indexical meaning». A sostegno di questa ipotesi, il panorama musicale cona temporaneo in lingua inglese offre alcuni interessanti suggerimenti: l'appropriazione di taluni caratteri fonetici propri dell'AAVE da parte di artisti rap e hip hop, come si riscontra in Iggy Azalea e in Meghan Trainor, ma soprattutto in Rihanna, suggerisce che tratti fonetici e prosodici propri di un gruppo idiolettico siano, infatti, acquisibili o modificabili ad hoc.

Uno studio pubblicato da Jones, Schellenberg e Gick (2017) analizza i tratti articolatori distintivi di cantanti indie-pop per rintracciare le cause di variazioni fonetiche e di pronuncia di questo genere. Secondo quanto individuato dagli autori, alcuni artisti potrebbero adoperare uno stile di canto faringalizzato che porta a variazioni vocaliche. I risultati dello studio mostrano un abbassamento generale delle zone di emissione vocalica tra parlato e cantato, dovuto ad una chiusura della zona faringea, che non avviene invece con back vowels (di per sé difficilmente riducibili) e che è sintomatico di questo stile musicale e, viene suggerito, 'necessario' ai fini dell'inclusione dell'artista stesso nel genere musicale indie. Per altre tipologie di testo di riferimento, antecedenti all'apparizione mainstream di que-

5 Suggeriamo, a questo proposito, la lettura di Motschenbacher 2016, che mette in luce le divergenze lessicali presenti nelle tipologie di musica pop analizzate. 
sta tipologia stilistica, come la pronuncia di myself in Believe di Cher (Believe, 1998), corrispondente a /mise:/, possiamo prendere in considerazione l'adozione di strumenti di sintesi musicale come causa possibile di modifica del messaggio.

Solamente inserendo le possibilità di variazione fonetica in un diagramma unico è possibile riconoscere che un'impronta ibrida è al cuore degli episodi rintracciabili nel panorama musicale, di cui si compongono in larga parte elementi concernenti la produzione di materiale fonatorio in rapporto con il sottofondo musicale, ed elementi di retroscena, bagagli culturali di origine e a cui attingere per la resa di un personale stile di canto [tab. 2].

Tabella 2 Elementi alla base di variazioni fonetiche

\begin{tabular}{ll}
\hline Caratteri metrici della canzone & $\begin{array}{l}\text { Categoria socio-fonetica } \\
\text { di appartenenza (caratteri } \\
\text { sociolinguistici) }\end{array}$ \\
\hline Elementi stilistici della canzone (genere & $\begin{array}{l}\text { Categoria socio-fonetica di diffusione } \\
\text { musicale, cf. lo studio sull'autenticità di } \\
\text { Schulze 2014) }\end{array}$ \\
rappresentare)
\end{tabular}

Una porzione di giornalismo musicale e un'altra più modesta di teoria sociolinguistica hanno messo in evidenza la tendenza di perfomer internazionali a rendere il proprio approccio fonetico all'esecuzione emulando lo standard fonetico americano (Konert-Panek 2017b). Allo stesso tempo, una certa letteratura analitica in materia di teoria del canto (Neuerburg 2012; Story 2004) ha affidato alla qualità e alla condizione di emissione fonetica un importante ruolo di consegna di materiale fonologico. Chiaramente la sociolinguistica può prendere in considerazione tali supposizioni perché risulti più efficiente $e$ selettivo il suo stesso lavoro investigativo. Possiamo individuare diverse tendenze alla variazione fonetica che, ampliando gli studi già proposti, tentano di offrire una matrice essenziale il più possibile completa per quei meccanismi alla base dello shifting fonetico della Commercial Contemporary Music:

a. esigenze fisiologico-performative dell'atto locutorio; riuniamo in questo gruppo i processi di variazione fonetica dovuti all'aspetto performativo. Sottolineiamo soprattutto il processo di impostazione articolatoria delle vocali insieme al tono della voce, che non resta lo stesso per tutto l'arco della canzone, entrambi plausibili candidati a contribuire al diverso sfruttamento degli spazi di articolazione.

b. modulazione ai fini metrici; si ha, ad esempio, quando del materiale fonetico viene rimosso o arricchito per mantenere una certa coerenza metrica. L'aggiunta o l'eventuale riduzio- 
ne di tessuto fonetico risulterebbe la tendenza strumentale più efficiente perché il prodotto fonetico possa confarsi benevolmente allo sfondo musicale (un processo definito textsetting).

c. modulazione ai fini stilistici; in questo gruppo sono raccolte le trasformazioni fonetiche che si rendono necessarie nel caso in cui si voglia ottenere un effetto stilistico in generale, come una rima o un'assonanza/consonanza che, proprio per la loro natura occasionale e non stabile, sono accolte dall'ascoltatore non come trasformazioni fonetiche ma come semplici modulazioni. Anche l'appartenenza ad un genere musicale può portare a modifiche del tessuto fonetico.

d. caratteri idiolettici e sociolinguistici dell'atto locutorio; questo gruppo contiene variazioni fonetiche e fono-sintattiche che hanno alla base cause dialettali e geografico-linguistiche. La maggior parte della teoria sociolinguistica si è finora focalizzata su questo peculiare aspetto (Trudgill 2009). Diversi suggerimenti sono stati offerti dagli studiosi perché si rispondesse ai quesiti irrisolti inerenti al TAA (Trans-Atlantic Accent): in quali percentuali è necessario ricondurre al GA tutta la produzione musicale in lingua inglese? Fin dove è possibile uniformare tendenze fonetiche proprie dello standard americano a quelle di socioletti minoritari che pur presentano le medesime caratteristiche? E infine, la cosiddetta predominanza dell'accento americano ha semplicemente fondamenti socioculturali oppure è attribuibile ad altri fattori inerenti alle possibilità articolatorie della produzione cantata?

\subsection{Variazione a fine articolatorio-performativo}

Con variazione a fine articolatorio-performativo ci riferiamo a quei casi di variazione fonetica dovuti alle esigenze di pronuncia, postura, uso del diaframma, ecc. Tali fattori, la cui riuscita è alla base di una corretta performance canora, possono essere modulati rispetto alla necessità del performer e sono totalmente da riferire al contesto situazionale dell'esperienza. Già nel 2015 Gibson, ad esempio, descriveva la variazione fonetica di /ai/ per 'I' presente nel secondo chorus della traccia I will always love you di Withney Huston (1992), in cui l'artista forza la pronuncia realizzandola come /aid/ (Gibson 2015, 34). Allo stesso modo, se focalizziamo l'attenzione sul terzo chorus possiamo notare una ridondanza della medesima variazione che colpisce la pronuncia di 'always' (/'o:lweız/ $\rightarrow$ /a:lweiz/), variazione che si giustifica come 'conseguenza' articolatoria per una coerente e ottimale emissione sonora. 
And I will always love you (/a:Ia:/)

I will always love you (/a:wila:lweiz/)

Tale variazione risulta tuttavia efficace anche ai fini stilistici, poiché permette di produrre con successo la ripetizione del gruppo /a:Ia:/, pur separato dalla consonante /w/ ma recepita come un continuum. Si consideri anche la prossima tipologia di variazione presa in analisi:

- modulazione delle /ə/: il fonema /ə/ sembra diffusamente (RP: /'əひvə(r)/) sostituito da altri fonemi (in primis $/ \Lambda /$ per ragioni di comodità performativa. Come attestato per la pronuncia di 'over' (RP: /'əuvə(r)/) nel middle eight di When Love Takes Over di David Guetta, interpretata da Kelly Rowland nel 2009:

When love takes over, over, over, over...

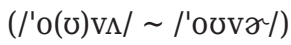

Le due forme fonetiche si alternano seguendo l'altezza della voce: la prima $(/ / \mathrm{o}(\circlearrowright) \mathrm{v} \Lambda /)$ si riscontra quando il tono della voce è più alto, la seconda (/'ouvr/) quando esso diminuisce. Ipotizziamo che questo tipo di variazione sia particolarmente apprezzabili nelle sezioni metriche finali del verso, quando il controllo sul prodotto fonetico e fonologico sembra venire meno, in versi più lunghi, o quando su di essi si posa tutta la forza fonologica, come accade al fonema $/ \partial /$, che viene sostituito da / / / in You're Beautiful di James Blunt (Back to Bedlam, 2004):

You're beautiful

You're beautiful

(/jor 'bjutəfیl/)

\subsection{Variazione a fine metrico}

Possiamo definire la variazione ai fini metrici come un processo di variazione del materiale fonetico dovuto a cause metriche, ovvero alla necessità di modulare l'emissione fonetica per mantenere un collegamento tra cantato e melodia (Nichols et al. 2009). In primo luogo, possiamo riscontrare una tendenza verso ciò che definiamo una hyper-articulation of $/ \mathrm{h} /$. Avviene per salvaguardare la prosodia del verso e ha come conseguenza diretta la creazione di un gruppo -/aha/ in White Mustang di Lana del Rey (Lust for Life, 2017):

The day I saw your white Mustang,

your white Mustang

(/ðə deı aı sə jə wahart ' $\mathrm{m} \Lambda_{1}$ stæy/

/jo wahaIt ' $m \Lambda_{1}$ stæy/) 
Un'altra tipologia di aggiunta di materiale fonetico (che definiamo qui epentesi vocalica) riguarda vocali di collegamento, ovvero fonemi privi di significato che sono utili a realizzare uniformemente la sequenza metrica del verso. In A pain that I'm used to dei Depeche Mode (Playing the Angel, 2005), l'inserimento della /a/ come vocale di collegamento permette maggiore fluidità metrica al verso:

All this running around, well it's getting me down just give me a pain that I'm used to (/pern ðæt arma-'-'ju:stu/)

Lo stesso fenomeno è riscontrabile nella sezione introduttiva, questa volta più lenta, di Violently Happy di Bjork (Debut, 1993), dove sono inseriti ben tre epentesi vocaliche (la schwa, la vocale centrale /a/ e il gruppo /ei/ per me):

Since I met you

This small town hasn't got room

(/sins ai me:tJju/

/ði:s smol -ə-taon//'h-a-æzənt ga:t ru:m/)

Si evidenziano, d'altra parte, casi di arricchimento fonetico in cui, pur col fine di sostenere la sillaba, il materiale aggiunto non modifica la qualità del suono successivo o anteriore emesso, ma viene inteso come un'aggiunta fonetica a sé stante, priva di significato, spesso ai fini della trascrizione, intesa come aggiunta esclamativa (oh, ah). Troviamo un esempio nel chorus di Promise This di Cheryl Cole (Messy Little Raindrops, 2010): «Promise this / If I die before I wake-oh», in cui la vocale in epentesi è una vera e propria aggiunta di carattere espressivo e non è da intendersi né come mispronunciation né tantomeno come una variazione della preposizione up.

Riscontriamo, inoltre, un'eliminazione di gruppi fonetici a fine metrico. Questo processo è proprio di pattern fonetici in cui non è possibile rispettare metricamente la corretta pronuncia di tutte le sillabe: si adopera una cesura del processo fonetico con conseguente riduzione o cancellazione di materiale fonologico. Come già individuato da Magnusson (2008), tale riduzione appare spesso nel rap e nell'hip-hop, come in Those Gurlz di Snoop Dogg (Ego Trippin', 2007):

(I know you remember me)

$\rightarrow$ I know you 'member me

O in Ni**as in Paris di Jay-Z e Kanye West (Watch the Throne, 2011):

(We aren't even supposed to be here)

$\rightarrow$ We ain't even 'pose to be here 
Altro fenomeno evidente riguarda la monottongazione dei dittonghi e trittonghi: spiegare il processo di riduzione dei dittonghi in unità monosillabiche o dittonghi deboli $\left(/ \mathrm{a}^{\mathrm{I}} /\right)$ può trovare una più coerente spiegazione se circoscritta nel più vasto gruppo di processi di modulazione fonetica che hanno alla base motivazioni metriche. Non sempre, infatti, la monottongazione è rispettata e spesso la scelta tra dittongo (ad esempio /ai/) completo e ridotto (/a $/ /)$ segue delle logiche interne alla presenza/assenza di peaks metrici nel verso ed è strettamente collegata al genere musicale della traccia. In Brooklyn Baby (soft rock) di Lana del Rey (Ultraviolence, 2011), ad esempio, la /i/ del gruppo /ei/ (/beibi/) fa parte del peak metrico e viene articolata correttamente, mentre in High by the Beach (pop/hip hop) della stessa cantante (Honeymoon, 2015) la pronuncia di baby - in questo caso al di fuori del peak metrico - corrisponde a /bebi/. Allo stesso modo agirebbe il processo di happy-tensing (alternanza tra allungamento e riduzione della pronuncia della vocale chiusa frontale) individuato dalla sociolinguistica comparativa (Wells 1982): l'estensione o la riduzione di un gruppo sillabico finale va rapportato alle possibilità metriche del verso analizzato.

\subsection{Variazione a fine stilistico}

Possiamo riscontrare variazioni nella pronuncia e idiosincrasie di natura stilistica, perfino aldilà di quanto presentato precedentemente in relazione all'accento indie contemporaneo. Questo tipo di variazione è spesso la meno inaspettata ma, al contempo, la più facilmente giustificabile da parte dell'ascoltatore. Riguarda soprattutto le vocali e i gruppi vocalici in funzione di assonanza e consonanza e ne modifica la qualità affinché le divergenze di pronuncia tra esse vengano sciolte e risolte in qualità convergenti. In Whatever It Takes degli Imagine Dragons (2017), ad esempio, la pronuncia di dangerous viene completamente modulata per creare una rima con this; ad essa si collega anche la modulazione vocalica del gruppo /-əs/ successiva:

Falling too fast to prepare for this (/ðIs/)

Tripping in the world could be dangerous (/'deindzəris/)

Everybody circling, it's vulturous (/'vəolt5əris/)

Negative, nepotist (/'negətıv/, /'nepə,tist/)

In Be the One di Dua Lipa (Be the One, 2015), riscontriamo la modulazione del fonema /mu:n/ (moon) in finale di parola, necessaria alla creazione di assonanza sia con sun (/s $\Lambda \mathrm{n} /)$ che con fool (/fu:l/):

I see the moon $(/ \mathrm{m} \Lambda \mathrm{n} /)$

I see the moon

I see the moon $(/ \mathrm{mu}: \mathrm{n} /)$ 
Oh, when you're looking at the sun (/sın/)

I'm not a fool (/fu:l/)

I'm not a fool

I'm not a fool

No, you're not fooling anyone (/عnIwın/)

McCulloch, linguista e scrittrice, individua nell'Indie Pop contemporaneo un processo di dittongazione automatica che caratterizza la pronuncia di alcuni monottonghi (aggiungiamo noi, in posizione tonica) e che non può essere interpretato se non come una variazione puramente stilistica, una vera e propria prestabilita mispronunciation. Nella collaborazione con Reggie Ugwu, giornalista per BuzzFeed, individua altri casi di questo tipo di variazione fonetica: ${ }^{6}$ riportiamo qui la modulazione della pronuncia di good (/god $\rightarrow / \mathrm{goId} /$ ) in Good for You di Selena Gomez feat. A \$AP Rocky (Revival, 2015) e quella di care (/keə/ $\rightarrow / \mathrm{k} \varepsilon \Lambda \mathrm{r} /$ ) in Royals di Lorde (Pure Heroine, 2013). Tali variazioni si potrebbero giustificare come iper-articolazioni, allo stesso modo delle aspirate viste in precedenza, originate però questa volta da cause stilistiche.

\subsection{Variazione di carattere idiolettico e sociolinguistico}

L'intuizione sociolinguistica di Labov (1963) sembra ancora valida: la variabilità fonetica di un artista, il suo attenersi a regole fonetiche proprie di una cultura maggioritaria e dominante (overt prestige) o il suo spingersi verso frontiere linguistiche minoritarie (covert prestige) sono da intendersi come fenomeni voluti, pur se a volte incoerenti e irregolari.

A questo proposito, riportiamo parte di una recensione di Alexis Petridis (The Guardian) per Semper Femina di Laura Marling (2017):

The only thing that doesn't sound assured is Marling's accent, which goes back and forth across the Atlantic like the QE2. One minute it's cut-glass English, the next it's drawling Laurel Canyon. At one juncture, it even seems to head off for a short break in the West Country: "It feels like they tart us," she sings on Next Time, by which she means "it feels like they taught us". The affectations are jarring but obviously deliberate [...]. But why she does so is an intriguing question. Is she playing characters? Adjusting her voice to suit the different musical backings? She art to tell us. ${ }^{7}$

6 https://www.buzzfeed.com/reggieugwu/what-is-indie-pop-voice.

7 https://www.theguardian.com/music/2017/mar/09/laura-marling-semper-femina-review-more-alarming. 
Se analizziamo il contesto fonetico della pronuncia constatiamo che la parola taught viene realizzata come /tor/ invece di /to:t/ (UK English). ${ }^{8}$ Questo carattere d'identità americana non viene riscontrato in Made by Maid (I Speak Because I Can, 2010), dove taught viene pronunciato in inglese standard. Il giornalista stesso, d'altra parte, suggerisce che questo effetto sia dovuto all'emozione che l'artista trasmette e tramite la quale tale parola viene pronunciata, ovvero una certa rabbia. La posizione dell'apparato articolatorio può portare a tanto evidenti effetti di pronuncia, non tanto su una vocale, quanto più su una vera e propria consonante? Se pensiamo che viene suggerito ai perfomer di simulare un sorriso per rendere più brillante il suono della propria voce, ${ }^{9}$ possiamo da una parte confermare questo aspetto teorico in merito alla performance canora, ma anche affermare il contrario, ovvero che un controllo emotivo sull'emissione fonetica può condurre a soluzioni divergenti dallo standard.

Essendo, però, il compito della sociolinguistica individuare il quid ultimo della produzione di un testo orale, non possiamo che riconoscere che ancora oggi, almeno in ambito musicale, sia particolarmente arduo individuare una teoria uniforme che sappia ricondurre ogni qualità di un testo in un più globale contesto fonetico di appartenenza. Già ponendo in primo piano l'occasione di esecuzione di un testo orale, come già visto, possiamo scorgere interessanti spunti di riflessione.

La sfida maggiore è, però, uniformare tutta la produzione musicale mainstream registrata, la cui occasione di esecuzione non prevede pubblico reale e il cui valore sociolinguistico si concentra nella personalità e nell'attitudine del performer e nello studio attuato da un'équipe di produzione. Non per questo dobbiamo restare inermi di fronte a certe evidenze fonetiche che assumono un valore di espressione di appartenenza socioculturale, o di intenzione ad appartenere ad un determinato contesto sociale di destinazione culturale. Possiamo riscontrare, tra le innumerevoli evidenze, casi di glottal stop in Galvanize dei Chemical Brothers (Push the Button, 2005), in cui la pronuncia di button diventa $/ \mathrm{b} \Lambda \mathrm{Pn} /$. Tale tendenza può essere assimilata alla pronuncia tipica del Cockney: gli artisti che compongono il duo sono entrambi nati a Londra. Sempre riguardo al dialetto londinese, diversi studi sono stati avanzati riguardo all'uso compiutone da Amy Winehouse e sul rapporto dell'artista riguardo l'esecuzione di una pronuncia standardizzata al GA (Konert-Panek 2017a). In Overshooting Americanisation della stessa autrice (2017), viene messa in luce la presenza di coda-r; LOT vowel unrounding; flapping e monottongazione del dittongo nel suo stile di canto più recente, $\mathrm{e}$

9 https://takelessons.com/blog/daily-vocal-exercises. 
non presenti nei primi album (2017a, 382). Tale variazione sarebbe attribuibile ad una necessità commerciale: adottare uno stile fonatorio standard per adattarsi ad un pubblico più mainstream. La sostituzione del fonema / / con lo standard /tt/ o addirittura la retroflessa americana sembra suggerire una capacità di shifting fonetico particolarmente adattivo alle esigenze di produzione, a riprova del fatto che, almeno nell'ambito della variazione per cause geograficodialettali, sarebbe più utile adoperare un metodo induttivo, piuttosto che deduttivo, ovvero tessere una tela normativa autonoma propria del TAH (Trans-Atlantic Hybrid) basata su peculiari regole fonetiche, cui talvolta si confanno le norme sociolettiche già note (British English e General American in rilievo).

Per altri artisti, tra cui Nial Tennat dei Pet Shop Boys, è difficile trovare una collocazione socio-fonetica definitiva. La spontanea ecletticità dell'artista - il cui accento viene definito 'Geordie ${ }^{\prime 10}$ - porta a soluzioni differenti: da una parte, in West End Girls, il frontman pronuncia better come /berr/, come nel GA; dall'altra, la loro produzione offre altri evidenti shifting dialettali: la pronuncia di boy come /buoI/ ${ }^{11}$ in Left to My Own Devices (Introspective, 1988); l'assenza di rotacismo in Can You Forgive her (Very, 1994) - in cui her è pronunciato come /h3:/ /ha:/ - e, per ragioni metriche, la presenza di happy tensing per memory (/memə'ri:/).

\subsection{Esempi di variazione geografico-dialettale}

Tra gli innumerevoli casi di variazione geografico-dialettale di interesse, prononiamo:

\section{a. Accento Essex nei Blur}

Il dialetto Essex (mescolato col cockney dell'East London) nei Blur, particolarmente notevole nei primi album (Leisure, 1991). Quello che intendiamo mettere in luce riguardo alle tendenze fonetiche del cantante del gruppo, Damon Albarn, è come esse siano spesso state interpretate come casi di Mockney, ovvero di falsificazione della pronuncia cockney. ${ }^{12}$

10 https://www.thesun.co.uk/archives/news/1100467/pet-shop-boys-this-isnot-a-comeback-or-return-to-form-because-weve-never-been-away.

11 Riguardo al Geordie, si veda Watt, Allen 2003, 268; la tendenza è comunque riscontrata anche nel GA: si veda Wells 1982, 486.

12 https://blogs.bl.uk/sound-and-vision/2018/06/london-dialect-in-pop-music.html. 


\section{b. Ipotesi di accento irlandese in Sinéad O'Connor}

La presenza di tracce fonetiche locali nella produzione di Sinéad O' Connor, seppur raramente evidente, può essere oggetto di studio. In Nothing Compares to You (I Do not Want What I Haven't Got, 1990) rileviamo la sostituzione del fonema $/ \Lambda /$ in fun. La pronuncia della parola appare chiusa e posta nella zona posteriore del palato. Ciò potrebbe coincidere con un'abitudine dialettale di pronuncia (ricordiamo che l'artista nasce a Dublino).

\section{c. Esempi di Black English}

Internamente alla distesa variabilità fonetica propria della lingua inglese, possiamo considerare il vernacolare afroamericano come la minoranza sociolinguistica di maggior rilevanza in merito al nostro studio sulla musica contemporanea. Quale realtà sociolettica autonoma, poiché esperienza linguistica secolare e maturata in contemporanea con la cultura di appartenenza e con le evoluzioni sociali interne ad essa e al suo rapporto con quella americana predominante (vale a dire quella bianca e di origine WASP), diversi aspetti qualitativi che le appartengono fanno pensare che il suo fascino non risieda meramente nella divergenza rispetto alle maggioritarie presenze linguistiche, quanto più nella sua possibilità di veicolare aspetti intenzionali fonetici, lessicali e sociali insiti nella cultura afroamericana stessa. L'AAVE è stato spesso, come abbiamo già suggerito, oggetto di appropriazione culturale da parte dei gruppi sociali maggioritari: gli episodi di style shifting e code switching rintracciabili in seno alla CCM suggeriscono con evidente realismo che l'universalità di tale apparato socio-fonetico non è limitato al gruppo sociale afroamericano, in quanto è stato col tempo universalizzato e generalizzato come strumento di identificazione culturale rispetto alla cultura suburbana. La storia della musica contemporanea sembra, d'altra parte, focalizzarsi fin da subito sulla diffusione culturale afroamericana e, ad oggi, diversi generi musicali sono di derivazione o di influenza black: si pensi al bepop, al jazz, all'RNB, all'hip-hop, al rap, alla trap.

Artisti e produttori di origine afroamericana sono tutt'oggi al vertice della creazione culturale musicale e in vetta alle classifiche degli artisti più remunerati della nostra epoca (Beyoncé, Kanye West, Jay-z, Tupac, Bob Marley, ecc.). Tanto è plausibile la tendenza (commerciale) ad assumere un accento americano nel canto, tanto è riscontrabile un moto continuo e ben poco uniforme di migrazioni e spostamenti da tale contesto, pur impercettibili o difficilmente gerarchizzabili che sia (del resto il nostro studio è limitato alla traccia registrata). Proprio in merito agli artisti di qualche decade fa è possibile interpretare l'uso del dialetto come strumento di coesione e appartenenza ad un gruppo etnico. Pensando a Rihanna Fenty, nata 
nel 1988 alle Barbados, ne riconosciamo una svolta dialettica particolarmente evidente negli ultimi anni. In Man Down (Loud, 2011) lo shifting fonetico da GA a Bajan è evidente alla fine del secondo chorus, quando nel cantato iniziano a subentrare fenomeni di evidente dentalizzazione, colpi di glottide, e modifiche in seno alla pronuncia delle vocali. In Work (Anti, 2016) l'uso del vernacolare creolo è ancora aumentato e risulta estremamente utile nella creazione di uno stile personale e carico d'orgoglio etnico e socioculturale.

\section{Conclusioni}

L'analisi sociolinguistica effettuata suggerisce diverse riflessioni riguardo alle modalità e alle necessità inerenti alla creazione di una teoria uniformata e coerente che prenda in analisi elementi di semiotica, linguistica pragmatica, sociolinguistica e studi linguistico-cognitivi. In qualità di oggetto culturale vero e proprio (Griswold 2012, 15), estremamente accessibile, sia nella trasmissione che nella diffusione, una canzone è testimonianza di società e storia culturale: solo riconoscendo quali e quanti elementi di significato e di referenza sono messi in atto all'interno di una traccia è possibile individuare le ragioni di tanta eterogenea variabilità interna, e per quali motivi molti di essi siano entrati a far parte della cultura storica occidentale.

Come precedentemente suggerito, l'ascolto e la memorizzazione di testi popolari ai fini dell'apprendimento dell'inglese come L2 (procedimenti positivamente classificati dalla linguistica e promossi dalla teoria didattica) possono essere inclusi in tale discorso: ci si chiede quali esiti sulla produzione fonetica e linguistica individuale dell'apprendente di inglese come L2 possa avere lo studio della musica popolare angloamericana e se esso finisca per comportare l'acquisizione di un sistema fonetico e linguistico standardizzato o di per sé settoriale. Riconosciamo che il lavoro affrontato è un lavoro in itinere, poiché inerente al mondo mediale contemporaneo, ed è ricco di sfide in quanto si propone di evidenziare cambiamenti in atto nella cultura del nostro tempo, suggerire piste di ricerca sociolinguistica che, applicando elementi di fonetica e linguistica pragmatico-cognitiva, finiscano per individuare i caratteri universali dell'applicazione didattica dell'espressione musico-testuale in lingua inglese, nonché la variabilità e l'uniformità insite in essa. Più che meramente riconoscere l'utilità della canzone pop nel contesto educativo di ogni livello ed età, arricchiamo la teoria linguistica in questo ambito riconfermando quei fattori di variation e style-shifting succitati e suggerendo possibili motivazioni di carattere occasionale-performativo, o geograficodialettale alla base delle idiosincrasie fonetiche. Tali innovativi elementi di analisi, quali la necessità di integrare fattori interni all'atto fonetico-articolatorio nel canto moderno e quelli dovuti a ragioni me- 
trico-stilistiche - rappresentano classi categoriche di ricerca particolarmente adattabili ai contesti educativi avanzati.

Ulteriori studi potrebbero sorgere dall'analisi dell'ottica sociale di provenienza di una canzone o in relazione al suo pubblico di riferimento e, nel caso esso fosse minoritario, come e quanto l'oggetto culturale preso come riferimento metta in evidenza caratteri fonetici, linguistici e semantici di una comunità non-standard. Bisognerebbe, d'altro canto, analizzare in parallelo ogni minoranza e il suo rapporto con una maggioranza di pubblico che potrà in seguito accettare e condividere, o rifiutare, il messaggio sociale espresso. Anche qui sarà necessario interrogarsi riguardo alle ragioni sociali del successo o dell'insuccesso di una canzone e da qui riconfermare la teoria di Trudgill, il quale vede nelle possibilità commerciali dell'industria musicale made in USA le ragioni di quello shifting fonetico che, se nel suo caso rappresentava un episodio innovativo, oggigiorno è tendenza quotidiana e universalmente accettata.

Laddove, poi, ci chiedessimo i perché delle diverse rese fonetiche inerenti a una canzone, dovremmo porre in relazione la storia di un testo e la biografia linguistica del suo autore, la sua conoscenza della dizione e chiederci se il suo cantato corrisponda foneticamente al suo parlato, se l'uso di elementi non-standard risulti costante nel tempo e nelle occasioni di realizzazione (potrebbe, d'altra parte rivelarsi situazionale: si veda Schulze 2014) e infine riconoscere il potere sociolinguistico di tali evidenze di variabilità. Pur essendo la ricerca nel nostro Paese ancora agli albori rispetto a questo ambito, non c'è da avvilirsi: finché avremo da ascoltare canzoni, la nostra ricerca potrà continuare.

\section{Bibliografia}

Anderson, C.A., Carnagey, N.L.; Eubanks, J. (2003). «Exposure to Violent Media. The Effects of Songs with Violent Lyrics on Aggressive Thoughts and Feelings». Journal of Personality and Social Psychology, 84(5), 960-71.

Asgautsen, R. (2017). American, British or Norwegian English? A Phonological Analysis of Songs by Norwegian Singers Sung in English [MA Thesis]. Bergen: University of Bergen.

Beal, J.C. (2009). "“You're not from New York City, You're from Rotherham”. Dialect and Identity in British Indie Music». Journal of English Linguistics, 37(3),

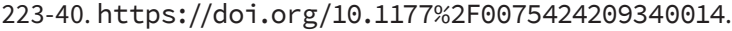

Boothe, D.; West, J. (2015). «English Language Learning through Music and Song Lyrics. The Performance of a Lifetime». The Future of Education = Conference Proceedings. 5th ed. (Florence, 11-12 June). Padova: Libreriauniversitaria.it, 63

Dubin, F. (1975). «An Overlooked Resource for English Language Teaching: Pop, Rock, and Folk Music». CATESOL (California Association of Teachers of English to Speakers of Other Languages) Occasional Papers, 2, 40-7. 
Eiter, A. (2017). "Haters Gonna Hate". A Corpus Linguistic Analysis of the Use of NonStandard English in Pop Songs [BA Thesis]. Innsbruck: University of Innsbruck.

Ferrante, J. [1992] (2014). Sociology. A Global Perspective. Belmont (CA): Cengage Learning.

Gibson, R.M. (2015).The Role of Accent in Popular Music. An Interdisciplinary Approach [MA Thesis]. Huddersfield: University of Huddersfield.

Griswold, W. (2012). Cultures and Societies in a Changing World. Sociology for a New Century Series. Los Angeles: SAGE.

Howe, D. (2005). Negation in African American Vernacular English [Masters thesis]. Calgary: University of Calgary.

Hughes, A.; Trudgill, P.E; Watt, D. (2005). English Accents and Dialects. An Introduction to Social and Regional Varieties of English in the British Isles. London: Hodder Arnold.

Israeli, H.F. (2013). «Language Learning Enhanced by Music and Song». Literacy Information and Computer Education Journal (LICEJ). Special Issue, 2(1), 1360-6.

Konert-Panek, M. (2017a). «Americanisation versus Cockney Stylisation in Amy Winehouse's Singing Accent». Kennedy, V.; Gadpaille, M. (eds), Ethnic and Cultural Identity in Music and Song Lyrics. Newcastle upon Tyne: Cambridge Scholars Publishing, 77-94.

Konert-Panek, M. (2017b). "Overshooting Americanisation. Accent Stylisation in Pop Singing. Acoustic Properties of the Bath and Trap Vowels in Focus». Research in Language, 15(4), 371-84. https://doi.org/10.1515/ rela-2017-0021.

Jones, C.; Schellenberg, M.; Gick, B. (2017). «Indie-Pop Voice: How a Pharyngeal/Retracted Articulatory Setting May Be Driving a New Singing Style». Canadian Acoustics - Acoustique Canadienne, 45(3), 180-1.

Labov, W. (1963). «The Social Motivation of a Sound Change». WORD, 1(3), 273309. https://doi.org/10.1080/00437956.1963.11659799.

Magnusson, M. (2008). Not Perfect Grammar, Always Perfect Timing. African American Vernacular English in Black and White Rap Lyrics [Bachelor Thesis]. Karlstads: Karlstads Universität.

McGregor, W. (2009). Linguistics. An Introduction. London; New York: Bloomsbury Publishing.

Middleton, R. (1990). Studying Popular Music. Milton Keynes: Open University Press.

Motschenbacher, H. (2016). «A Corpus Linguistic Study of the Situatedness of English Pop Song Lyrics». Corpora, 11(1), 1-28. https://doi.org/10.3366/ cor.2016.0083.

Mauch, M. et al. (2015). «The Evolution of Popular Music. USA 1960-2010». Royal Society Open Science, 2(5), 150081. https://doi.org/10.1098/ rsos.150081.

Neuerburg, T. (2012). The Impact of Vowels on Pitch Finding and Intonation in the Movable-Do Solmization System. Student Research in Creative Activity, and Performance - School of Music, 51. University of Nebraska, Lincoln. https://digitalcommons.unl.edu/musicstudent/51.

Nichols, E.; Morris, D.; Basu, S.; Raphael, C. (2009). «Relationships Between Lyrics and Melody in Popular Music». Proceedings of the 10th International Society for Music Information Retrieval Conference (ISMIR 2009) (Kobe, Japan, October 26-30, 2009). S.I.: International Society for Music Information Retrieval, 471-6. https://www.microsoft.com/en-us/research/publication/relationships-lyrics-melody-popular-music/. 
Nishina, Y. (2016). «Building a Theoretical Model for the Usefulness of Pop Culture Teaching Materials». JACET Kansai Journal, 18, 52-65.

Nishina, Y. (2017). «A Study of Pop Songs Based on the Billboard Corpus». International Journal of Language and Linguistics, 4(2), 125-34.

Ranzato, I. (2016). «Dialects in the L2 Classroom. Teaching Regional and Social Varieties of English through Screen Adaptations». Di Stefano, F.; Ghia, E.; Marcucci, G. (a cura di), Dallo schermo alla didattica di lingua e traduzione: otto lingue a confronto. Pisa: Edizioni ETS, 89-105.

Ranzato, I. (2019). «Gayspeak in the Translation Classroom». de Marco, M.; Toto, P. (eds), Gender Approaches in the Translation Classroom. Training the Doers. London: Palgrave Macmillan, 103-26.

Richards, J.C. (1969). «Songs in Language Learning». TESOL Quarterly, 3, 161-74.

Schulze, C. (2014). Identity Performance in British Rock and Indie Music: Authenticity, Stylization, and Glocalization [Unpublished Master's Thesis]. Lund: Lund University.

Schwartz, M. (2013). «Learning with Lady GaGa \& CO. Incidental EFL Vocabulary Acquisition from Pop Songs». VIEWS, 22, 17-48.

Simpson, P. (1999). «Language, Culture and Identity. With (Another) Look at Accents in Pop and Rock Singing». Multilingua, 18(4), 343-68. https://doi. org/10.1515/mult.1999.18.4.343.

Story, B. (2004). «Vowel Acoustics for Speaking and Singing». Acta Acustica united with Acustica, 90(4), 629-40.

Trudgill, P. (1983). «Acts of Conflicting Identity». Trudgill, P. (ed.), On Dialect Social and Geographical Perspectives. New York; London: New York University Press, 141-60.

Trudgill, P. (2000). Sociolinguistics. An Introduction to Language and Society. London: Penguin.

Trudgill, P. (2009). «Vernacular Universals and the Sociolinguistic Typology of English Dialects». Filppula, M.; Klemola, J.; Paulasto, H. (eds), Vernacular Universals and Language Contacts. Evidence from Varieties of English and Beyond. London: Routledge, 304-21.

Watt, D.; Allen, W. (2003). «Illustrations of the IPA: Tyneside English». Journal of the International Phonetic Association, 33(2), 267-71.

Wells, J. (1982). Accents of English. Cambridge: Cambridge University Press.

Williams, J. (2014). "'Same DNA, but Born this Way". Lady Gaga and the Possibilities of Postessentialist Feminisms». Journal of Popular Music Studies, 26(1), 28-46. https://doi.org/10.1111/jpms.12058. 


\title{
EL.LE
}

Vol. 9 - Num. 1- Marzo 2020

\section{Lo sviluppo della competenza traduttiva e l'uso della traduzione nella didattica delle lingue moderne}

\author{
Ruggiero Pergola \\ Università degli Studi di Bari «Aldo Moro», Italia
}

\begin{abstract}
In everyday communication, both in specialized and non-specialized contexts, translation plays a pivotal role. Although it was considered an outsider in language teaching for decades, translation has now been rehabilitated and should now be considered a major component for language teachers and students. This paper addresses the development of translation competence and the use of translation in teaching and learning modern languages and analyses the main theories and models which describe the acquisition of translation competence in translation training.
\end{abstract}

Keywords Language teaching. Second language learning. Translation competence. Translation training. Translation in language teaching.

Sommario 1 Tradurre: tra competenze e uso glottodidattico. - 2 La competenza traduttiva. - 3 Un modello di competenza traduttiva. - 4 L'acquisizione della competenza traduttiva. -5 La visione della traduzione in glottodidattica. -6 Le ragioni a favore della traduzione nell'insegnamento delle lingue straniere.

$\begin{array}{lll} & \text { Peer review } & \\ \text { Edizioni } & \text { Submitted } & 2019-12-15 \\ \text { Ca'Foscari } & \text { Accepted } & 2020-05-10 \\ & \text { Published } & 2020-06-23 \\ & \text { Open access } & \end{array}$

(ㄷ) 2020 | () Creative Commons Attribution 4.0 International Public License 


\section{Tradurre: tra competenze e uso glottodidattico}

La traduzione continua ad avere un ruolo secondario nell'insegnamento delle lingue straniere, nonostante una parte della glottodidattica più recente ne sostenga l'utilità come strumento di apprendimento. Dopo un lungo periodo di oscurantismo, peso e centralità della traduzione sono stati finalmente riconsiderati (cf. Malmkjær 1998, 2004; Cook 2010; Pym, Malmkjær, Gutiérrez-Colón Plana 2013), ma resta tuttora diffuso il pregiudizio secondo cui il saper tradurre costituirebbe «un corollario 'naturale' della conoscenza linguistica (se sai la lingua straniera, sei capace di tradurre) e non richieda, perciò, una programmazione didattica mirata e sistematica» (Mazzotta $2007,134)$. Tale condizione rappresenta forse una spia della limitata attenzione scientifica riservata dalla glottodidattica al fenomeno della traduzione. Nel presente scritto il discorso muoverà in due diverse direzioni: da una parte si analizzeranno le teorie e i modelli che descrivono l'acquisizione della competenza traduttiva, dall'altra si proverà a valorizzare ulteriormente la pratica della traduzione quale strumento didattico.

\section{La competenza traduttiva}

Nel suo erudito Le Ton Beau de Marot (1997) lo scienziato cognitivo Douglas Hofstadter - autore nel 1979 di uno dei testi più affascinanti sull'intelligenza artificiale, Gödel, Escher, Bach: un'eterna ghirlanda brillante - ha esplorato il significato profondo della traduzione, coi relativi punti di forza e di debolezza, per provare a tracciare una mappa inedita del linguaggio e della mente. Il tutto parte da una breve poesia di Clément Marot del 1537, intitolata A une Damoyselle Malade, che è dedicata quale augurio di pronta guarigione a una giovinetta di nome Jeanne d'Albret:

Ma mignonne,

Je vous donne

Le bon jour;

Le séjour

C'est prison.

Guérison

Recouvrez,

Puis ouvrez

Votre porte

Et qu'on sorte

Vitement,

Car Clément

Le vous mande. 
Va, friande
De ta bouche,
Qui se couche
En danger
Pour manger
Confitures;
Si tu dures
Trop malade,
Couleur fade
Tu prendras,
Et perdras
L'embonpoint.
Dieu te doint
Santé bonne,
Ma mignonne.

Hofstadter, che con un acuto anagramma sostiene che tradurre equivale a «perdersi in un'arte» (translation = lost in an art), decide di cimentarsi nella traduzione del brano in inglese e chiede di fare lo stesso a parenti, amici e colleghi, precisando che requisito imprescindibile è il rispetto dei seguenti vincoli della struttura metrica: 28 versi; versi di tre sillabe; l'accento di ogni verso sull'ultima sillaba; la rima baciata di ogni distico; il passaggio dal formale vous all'informale $t u$; l'uguaglianza del primo e dell'ultimo verso; l'inserimento del nome del poeta a circa metà dell'opera. Il risultato finale che ottiene dopo alcuni mesi ammonta a ben 88 versioni, da quella più letterale a quella in stile rap, in stile elisabettiano, ecc. in un continuum che ricorda i noti Exercices de style di Queneau. Tutte versioni diverse, ma tutte più o meno accettabili, eseguite, oltre che da lui stesso, da una moltitudine di figure che spaziano da chi traduce professionalmente fino ai software informatici. La variabilità delle traduzioni, concludeva Hofstadter, è forse tra le migliori prove della sconfinata creatività della mente umana.

Dalla nascita dei Translation Studies, che segna il secondo Novecento, ad oggi si è registrato un notevole impulso nella riflessione sulla traduzione e i problemi teorici e pratici legati a questo settore di ricerca, quali l'utilizzo delle nuove tecnologie informatiche, la critica delle traduzioni, la metodologia di insegnamento della traduzione e la formazione di chi traduce, hanno assunto una rilevanza senza precedenti, tanto da diventare argomento di un numero crescente di pubblicazioni internazionali (cf. Malmkjær, Windle 2011; Millán, Bartrina 2012; Baker, Saldanha 2020). Il tratto che maggiormente caratterizza il pensiero odierno è l'inquadramento della traduzione nel fenomeno più ampio della comunicazione interculturale (Bassnett 2002), che porta a prestare attenzione a fattori come la situazione dello scambio comunicativo, la personalità e il ruolo degli interlocutori, le pre- 
supposizioni, le intenzioni per cui si comunica, ecc. La valorizzazione del legame tra la lingua di un popolo e la sua cultura fa maturare la visione di chi traduce come mediatore socioculturale tra l'autore del testo di partenza e il lettore di arrivo, per cui le conoscenze relative ad usi, costumi, valori, credenze e stereotipi condivise dagli appartenenti alla comunità linguistica a cui il testo è destinato diventano di fondamentale importanza. Su questa linea si muove Arcaini $(1992,16)$, che descrive la traduzione come un'operazione complessa, radicata nel tempo e nello spazio, che consiste nel trasferire un'unità della comunicazione - il testo - dalla cultura di partenza alla cultura di arrivo, tenendo conto dei destinatari. Altri preferiscono parlare della traduzione come «processo» (Pierini 2001, 17), ossia un'attività che implica una pluralità di operazioni diverse, condensate in due fasi principali: comprensione e interpretazione del testo di partenza e scrittura del testo di arrivo. Secondo Bassnett $(2002,18)$, questi due orientamenti sono riconducibili a un sistema bipolare focalizzato ad un'estremità sul prodotto e all'altra sul processo.

Parimenti impegnativo risulta il ruolo di chi traduce, che, nella sua doppia veste di ricevente e produttore, deve mediare fra lingue e culture, con un compito che comporta una responsabilità etica, oltre che scientifica, legata all'adeguamento di un testo alle esigenze dei suoi successivi fruitori (Lefevere 1975, 53). Di chi traduce si può dire che legge per sé e per gli altri (Arcaini 1992, 16). Nella fase d'interpretazione, infatti, la padronanza della lingua interagisce con la conoscenza della cultura nell'analisi sia interna al testo sia esterna, cioè relativa al contesto socio-situazionale di produzione; nella fase di riformulazione/riscrittura, invece, la padronanza di una lingua diversa interagisce con la conoscenza della cultura, così da realizzare un testo finale che non sembri semplicemente una buona replica di quello precedente, ma abbia la stessa efficacia comunicativa di un testo prodotto direttamente in quella lingua e sia conforme agli stessi criteri che ne decidono la qualità nella cultura di accoglienza (Arcaini 1991, 85-95). Attraverso interventi di compensazione linguistica e/o culturale si viene a stabilire, insomma, una equivalenza pragmatica tra testi che, pur differenti in superficie, conservano i livelli semantici e comunicativi profondi.

In quest'ottica, nella formazione di chi traduce assume un ruolo di primo piano lo sviluppo della competenza traduttiva, in quanto mezzo per realizzare un valido prodotto (il testo di arrivo) nonché obiettivo didattico. La centralità della competenza traduttiva negli attuali filoni di ricerca è stata richiamata in più occasioni: Malmkjær (2009, 122) sostiene, infatti, che «the notion of translation competence is central in translation theory as well as in pedagogical approaches to translation» e Saldanha e O'Brien $(2013,112)$ sottolineano che «by far the largest focus in translation process research to date [...] has been translation competence and its acquisition». 
Utilizzato per descrivere l'insieme di conoscenze, abilità, capacità e atteggiamenti in possesso di chi traduce professionalmente e che intervengono nel caratterizzare la traduzione come attività esperta, il concetto di 'competenza' applicato alla traduzione è piuttosto recente. Se, per esempio, in sociolinguistica Hymes (1971) ha iniziato a parlare di «competenza comunicativa» già negli anni Sessanta del Novecento, il termine - con la sola eccezione di Wilss (1976) - inizia a essere impiegato in traduzione soprattutto a partire dalla seconda metà degli anni Ottanta, anche se assume denominazioni via via sempre diverse nei vari autori, cosa che mostra l'evidente incertezza nella scelta di una terminologia univoca. Per citare solo i casi più rappresentativi, si parla di «Translation Proficiency» (Wilss 1982), «Translation Competence» (Toury 1984), «Transfer Competence» (Nord 1992), «Translator's competence» (Kussmaul 1995), «Translation Performance» (Wilss 1998; Rothe-Neves 2007).

Occorre altresì rilevare che i vari autori che parlano di competenza traduttiva mancano tuttavia di fornirne una definizione esplicita. Le uniche eccezioni sono le seguenti, accomunate tutte dall'uso di parole-chiave come knowledge, skills e abilities per descrivere ciò che avviene all'interno dei processi di traduzione:

- «An interlingual supercompetence [...] based on a comprehensive knowledge of the respective SL and TL including the textpragmatic dimension, and consists of the ability to integrate the two monolingual competencies on a higher level» (Wilss 1982, 58);

- «Knowledge and skills the translator must possess in order to carry it out» (Bell 1991, 43);

- «The ability of knowing how to translate» (Hurtado Albir 1996a, 48);

- «The underlying system of knowledge and skills needed to be able to translate» (PACTE 2003, 44).

\section{Un modello di competenza traduttiva}

Condizione necessaria di qualunque competenza traduttiva non può che essere un'adeguata competenza linguistica; questa è però necessaria ma non sufficiente. I primi modelli di competenza traduttiva si affermano insieme alle prime definizioni sopra riportate. Gran parte di queste proposte si limita a scomporre la competenza traduttiva in sotto-elementi (cf. per es. Bell 1991; Neubert 1994; Kiraly 1995; Hurtado Albir 1996a, 1996b; Risku 1998). Tra i vari modelli proposti, quello di Bell (1991) occupa sicuramente un posto di primo piano, anche per l'indubbio ruolo di apripista svolto nel panorama teorico. Egli suggerisce che la competenza traduttiva può caratterizzarsi per tre aspetti: una competenza bilingue ideale, che 
considera chi traduce un sistema idealizzato impeccabile; un sistema esperto, che poggia su una base di conoscenze e su un meccanismo di inferenza; e infine una competenza comunicativa, che comprende componenti grammaticali, sociolinguistiche, di discorso e strategiche (Bell 1991, 38-41).

Da questi primi modelli si ricava che:

- la competenza traduttiva richiede altre competenze oltre quelle linguistiche;

- include componenti diverse (conoscenze linguistiche ed extralinguistiche, capacità di documentazione, abilità nell'uso di strumenti, competenza di transfer, ecc.);

- e di diverso tipo (conoscenze, abilità, capacità, attitudini);

- esistono differenze fra traduzione diretta e inversa (cf. Hurtado Albir 2015, 258).

Successivamente si sono affermati ulteriori modelli, basati per la prima volta su sperimentazioni empiriche, dai quali emerge un concetto di competenza traduttiva intesa come conoscenza esperta che poggia su conoscenze dichiarative e procedurali. Alcuni di questi modelli vanno in direzione didattica (Kelly 2005; González Davies 2004), altri si basano sulla teoria della pertinenza (Gutt 2000), altri ancora prendono le mosse dagli studi sull'expertise (Shreve 2006; Göpferich 2009), da quelli sulla gestione della conoscenza (Risku, Dickinson, Pircher 2010) e dal punto di vista professionale e comportamentalista (Gouadec 2007). Uno dei modelli più completi, nonché il più adoperato negli studi, è quello messo a punto da PACTE (Process of Acs quisition of Translation Competence and Evaluation), un gruppo di ricerca fondato nel 1997 presso l'Universitat Autònoma di Barcellona con la precisa finalità di studiare la competenza traduttiva e la sua acquisizione (PACTE 2000, 2003; cf. anche Hurtado Albir 2017). Il modello prevede cinque sotto-competenze:

1. Una sotto-competenza bilingue: conoscenze principalmente procedurali richieste per comunicare tra due lingue;

2. Una sotto-competenza extralinguistica: conoscenze principalmente dichiarative sia implicite che esplicite riguardo il mondo in generale;

3. Una sotto-competenza sulla traduzione: conoscenze principalmente dichiarative sia implicite che esplicite riguardo la traduzione e gli aspetti della professione;

4. Una sotto-competenza strumentale: conoscenze principalmente procedurali relative all'uso delle fonti di documentazione e delle tecnologie per la traduzione;

5. Una sotto-competenza strategica: conoscenze principalmente procedurali richieste per accertarsi dell'efficienza dei processi traduttivi e per risolvere eventuali problemi. 
Alle sotto-competenze appena citate si aggiungono poi le seguenti componenti psicofisiologiche: componenti cognitive come memoria, percezione, attenzione, emozione; aspetti attitudinali come curiosità intellettuale, perseveranza, rigore, spirito critico, conoscenza e fiducia nelle proprie capacità, conoscenza dei propri limiti, motivazione; meccanismi psicomotori; abilità come creatività, ragionamento logico, analisi, sintesi (PACTE 2003, 58).

Gli studi del Gruppo PACTE consentono di trarre le seguenti conclusioni:

- La competenza traduttiva è una competenza acquisita distinta dalla competenza bilingue;

- La competenza traduttiva influenza il processo traduttivo e il suo prodotto (qualità della traduzione);

- La sotto-competenza strategica è fondamentale;

- Esistono differenze relative alla direzionalità (traduzione diretta/inversa) (cf. Hurtado Albir 2015, 259).

\section{$4 \quad$ L’acquisizione della competenza traduttiva}

Come per la definizione stessa di 'competenza traduttiva', esistono diversi modelli che ne descrivono l'acquisizione. Se, in linea generale, la gran parte degli studiosi vede un legame indissolubile tra preparazione teorica e addestramento alla professione, tra competenza cognitiva e operativa (quest'ultima costituita da saperi dichiarativi e procedurali), non manca chi ha sostenuto l'ipotesi, oggi ritenuta poco credibile e rigettata nella sua forma estrema, di un'attitudine innata alla traduzione. Harris $(1977,1980)$, pioniere di questa prospettiva, ritiene che qualunque studio empirico sulla traduzione non possa che partire da quei traduttori inconsapevoli che sono i bambini bilingui, prendendo come modello di riferimento la «traduzione naturale»: si tratta di quella forma di traduzione spontanea effettuata dai bambini fin dalla tenera età in situazioni naturali e senza alcuna formazione specifica. In questo caso, la competenza traduttiva aumenterebbe automaticamente con l'evolversi nel bambino dell'abilità di uso delle due lingue (Harris, Sherwood 1978, 160).

Ma se una qualche forma di predisposizione innata alla traduzione può anche esistere, comunque essa non determinerebbe lo sviluppo automatico della competenza traduttiva, la quale deve essere invece costruita mediante l'insegnamento delle norme di traduzione condivise da un dato ambiente socioculturale e in un preciso momento storico: la bravura di chi traduce dipenderebbe dalla interiorizzazione, attraverso l'istruzione e l'esperienza, di tali norme (Toury 1995, 241-58).

A partire da tali considerazioni sulla competenza traduttiva, vari autori hanno sviluppato diversi modelli e approcci teorici che hanno in comune il duplice aspetto della decodifica del testo di partenza e 
della produzione del testo nella lingua di arrivo; tra essi ricordiamo in particolare Toury (1995), Shreve (1997), Chesterman (1997) e per l'appunto PACTE (2000).

Toury (1995) inquadra la questione da un punto di vista sociologico, sostenendo che l'acquisizione delle norme, che regolano tutti i processi traduttivi, avviene durante un processo di socializzazione di chi traduce:

Socio-culturally speaking, what emerging translators thus undergo is socialization as concerns translating. During this process, parts of the normatively motivated feedback they receive are assimilated by them, modifying their basic competence and gradually becoming part of it. At every phase of its development, a native translator's 'competence' therefore represents a characteristic blend of nature and nurture, of the humanly innate, the individually assimilated and the socially determined. (Toury 1995, 250-1)

Shreve (1997) considera invece il processo di acquisizione della competenza traduttiva come un percorso compiuto dallo studente, che parte dalla «traduzione naturale» (Harris 1977) per arrivare alla cosiddetta «traduzione costruita», composita, complessa, a cui si perviene solo tramite una lunga esperienza; la traduzione professionale è definita come «a form of constructed translation that can be acquired by only undergoing certain kinds of deliberately sought out communicative experiences» (Shreve 1997, 125). Basandosi su H.L. Dreyfus e S.E. Dreyfus (1986), Chesterman (1997, 147-9) ha proposto invece un modello che identifica cinque stadi nella formazione di chi traduce (novizio, principiante avanzato, competente, abile ed esperto). Di grande interesse è infine il modello dinamico messo a punto dal Gruppo PACTE $(2000,2003)$, cui già sopra si faceva riferimento, che definisce l'acquisizione della competenza traduttiva come:

1. A dynamic, spiral process that, like all learning processes, evolves from novice knowledge (pre-translation competence) to expert knowledge (translation competence); it requires learning competence (learning strategies) and during the process both declarative and procedural types of knowledge are integrated, developed and restructured;

2. A process in which the development of procedural knowledge and, consequently, of the strategic sub-competence, are essential;

3. A process in which the translation competence sub-competences are developed and restructured. (PACTE 2003, 49-50)

In questo modello va messo in luce che le sotto-competenze sono in relazione tra loro e si compensano a vicenda; non si sviluppano necessariamente in parallelo; sono organizzate gerarchicamente; pre- 
sentano variazioni in relazione alla direzione della traduzione (diretta o inversa), alle combinazioni di lingue, alla microlingua (legale, letterario, ecc.) e al contesto di apprendimento (formale, autodidattico, ecc.) (PACTE 2003, 50).

Nel novembre 2011, lo stesso Gruppo PACTE ha condotto un esperimento che ha visto la partecipazione di 130 studenti di traduzione e interpretariato dell'Universitat Autònoma di Barcellona (PACTE 2014, 2015). I dati analizzati mostrano che:

- La competenza traduttiva è una competenza acquisita che si evolve con il progredire dell'apprendimento;

- L'acquisizione della competenza traduttiva influenza il processo di traduzione e il suo prodotto;

- L'acquisizione della competenza traduttiva implica il passaggio da un concetto e un approccio alla traduzione di tipo statico a uno di tipo dinamico;

- In tale passaggio, il più alto grado di dinamismo corrisponde alla conoscenza procedurale;

- Quando la competenza traduttiva viene acquisita c'è un aumento dell'uso combinato di supporti interni (risorse cognitive) e di vari tipi di risorse esterne;

- Le nuove generazioni utilizzano le risorse documentarie più frequentemente e più efficacemente (cf. Hurtado Albir 2015, 260-1).

\section{$5 \quad$ La visione della traduzione in glottodidattica}

Nella tradizione scolastica e accademica italiana (e non solo) la traduzione ha rivestito un ruolo di secondaria importanza nel quadro generale dell'apprendimento delle lingue straniere (Titone 1986; Cook 2010) e a lungo è stata ritenuta un'operazione meramente linguistica di trasferimento di significati da una lingua in un'altra; una convinzione che ha avuto notevoli ripercussioni anche sulla messa a punto di efficaci modelli didattici per insegnare a tradurre (Vermeer 1998, 60). All'incirca fino alla metà del Novecento, o comunque finché il metodo grammaticale-traduttivo è rimasto in auge nelle nostre scuole e università, il tradurre è stato concepito esclusivamente come un esercizio di verifica delle conoscenze grammaticali, per cui la traduzione consisteva nella resa pedissequa del testo di partenza e non era affatto inclusa tra gli obiettivi didattici. Ciò non tiene tuttavia in debito conto che:

La padronanza della lingua straniera rappresenta [...] uno dei requisiti dell'abilità traduttiva, ma al tempo stesso può essere approfondita e perfezionata per mezzo della traduzione, perché nelle operazioni metalinguistiche implicate dall'attività del tradurre si esalta la funzione cognitiva della lingua straniera come strumento di definizione dell'esperienza. (Mazzotta 2008, 117) 
Con l'affermarsi degli approcci comunicativi viene bandito l'uso della traduzione come mezzo di insegnamento delle lingue straniere e bisognerà attendere l'inizio del nuovo millennio perché il ruolo della traduzione nella glottodidattica cominci, seppur timidamente, a essere riconsiderato (cf. Malmkjær 1998, 2004; Cook 2010; Pym, Malmkjær, Gutiérrez-Colón Plana 2013; sulla situazione in Italia, Balboni 2007, 2010; Di Sabato 2007). Zojer sostiene che:

it is time to shift the discussion away from the theoretical level and move on to the practical one in order to discuss methods and strategies of how translation could be used to help language teachers and to benefit language learners. $(2009,42)$

Cook, deciso a riportare in primo piano la traduzione, ha persino parlato esplicitamente di «Translation in Language Teaching» (TILT) come nuovo paradigma didattico, evidenziando come «being able to translate is a major component of bilingual communicative competence» (Cook 2010, XX).

\section{Le ragioni a favore della traduzione nell'insegnamento delle lingue straniere}

Nel difendere l'uso della traduzione come strumento per l'insegnamento delle lingue, Balboni indica tre pilastri concettuali ai quali ancorare la discussione:

1. La traduzione mira a produrre metacompetenza linguistica e culturale: ovvero non produce direttamente competenza, se non quale effetto collaterale, ma metacompetenza; la finalità non è quindi la competenza d'uso ma sull'uso delle due lingue coinvolte;

2. La traduzione sviluppa i processi controllati e non quelli automatici: ovvero, richiamando Krashen, i processi consapevoli legati all'apprendimento e non quelli inconsci connessi con l'acquisizione;

3. La traduzione sviluppa la capacità di scoperta induttiva di una lingua straniera: ovvero è uno strumento per apprendere autonomamente lingue tipologicamente vicine tra loro (cf. Balboni 2010, 185-7).

Una prospettiva alternativa a Balboni è quella di Di Martino (2012), che invece sostiene che la traduzione può effettivamente promuovere la competenza d'uso della lingua, e non solo la metacompetenza, attraverso esercizi specifici mirati allo sviluppo o al perfezionamento della competenza linguistico-comunicativa in percorsi specifica- 
mente dedicati all'insegnamento e all'apprendimento della lingua. A tal proposito va sottolineato che:

Un utilizzo efficace di esercizi di traduzione nella classe di lingua ha come prerequisito la consapevolezza, da parte del docente, dei vantaggi che questa pratica apporta, perlomeno con apprendenti di livello avanzato: la traduzione va intesa come un ambiente di apprendimento sinceramente comunicativo, efficace per la promozione della competenza comunicativa in senso lato; una preziosa occasione per lo sviluppo della competenza interculturale; nonché un valido strumento per l'acquisizione della padronanza lessicale e per lo sviluppo della conoscenza della grammatica. (Di Martino 2012, 309)

La studiosa definisce «Circolo Virtuoso della Traduzione» il complesso processo educativo soggiacente: si parte da un testo (per il quale sono necessarie abilità di comprensione scritta, conoscenze grammaticali e lessicali nella L2/L1) per arrivare a creare un altro testo (per il quale sono necessarie abilità di produzione scritta, conoscenze grammaticali e lessicali nella L1/L2), entrambi calati nel mondo. Le singole abilità necessarie alla comprensione del testo in L1/L2 e alla produzione del testo in L2/L1 sono potenziate e arricchite durante il processo traduttivo, insieme alla conoscenza generale del mondo, e quindi alla cultura, implicite nella lingua dei due testi (Di Martino 2012, 310-11).

In merito alla presenza della traduzione ai livelli iniziali di studio di una lingua straniera si registra tuttavia ancora una certa cautela. Balboni $(2007,58)$, per esempio, ha sostenuto che «fino alla padronanza di un livello B1 la traduzione non ha senso, anzi instaura dei vizi mentali che rallentano l'acquisizione e l'uso della lingua». Di altro parere Di Martino e Di Sabato (2012), che sono favorevoli a un uso intelligente della traduzione nelle fasi iniziali di apprendimento di una lingua: dalla loro analisi di due documenti cruciali nell'apprendimento e nell'insegnamento delle lingue straniere - il Quadro comune europeo di riferimento per le lingue (Consiglio d'Europa 2002) e le Indicazioni per il curricolo della scuola dell'infanzia e del primo ciclo di istruzione (Ministero della Pubblica Istruzione 2007) - emerge infatti una forte presenza della traduzione, in tutte le forme e a tutti i livelli, che ne conferma l'impiego in chiave glottodidattica. Lo stesso Quadro evidenzia come la competenza linguistico-comunicativa (cf. Mazzotta 2002) si esplichi in macro-abilità nel compiere attività linguistiche che coinvolgono la ricezione (leggere, ascoltare), la produzione (scrivere, parlare), l'interazione (attività ricettive e produttive per la costruzione di un discorso comune) e, infine, proprio la mediazione (che comprende quella orale, interpretariato, e quella scritta, la traduzione). 
In classe, le modalità di realizzazione della traduzione sono molteplici e vanno dalla traduzione scritta dalla lingua straniera all'italiano alla traduzione 'all'impronta' dalla lingua straniera scritta o orale all'italiano orale, alla traduzione dall'italiano alla lingua straniera (estremamente difficile, ma utile come esperienza didattica se limitata a poche esercitazioni), alla traduzione di testi non solo verbali (canzoni, film, ecc.), alla retroversione, alla traduzione diacronica (che consiste nell'attualizzare un testo classico, come per esempio la Divina commedia, in italiano contemporaneo), alla traduzione da LS1 a LS2 (Balboni 2010, 190-6).

In conclusione, da quanto si è detto in precedenza emergono due diversi aspetti. Da un lato, la competenza traduttiva si acquisisce nel tempo grazie a un'intensa pratica, anche su diversi tipi di testi, e al concorso di molteplici saperi e saper fare, tra i quali riveste un ruolo particolarmente cruciale l'ampliamento delle conoscenze relative al background culturale della L1 e della LS. Tale competenza non può tuttavia essere lasciata maturare per via naturale, ma è necessario un intervento didattico organico: un corso universitario, per esempio, dovrebbe fornire una preparazione di base tale da sviluppare in chi dovrà tradurre l'abilità di acquisire ulteriore expertise attraverso la documentazione e l'elaborazione di propri strumenti professionali (Shreve 1997, 125). Dall'altro, la traduzione andrebbe rivalutata come strumento di apprendimento delle lingue straniere in quanto, come sottolinea Balboni, quando si lavora in aula con la traduzione scritta dalla LS all'italiano:

Questo tipo di traduzione focalizza l'attenzione sia sulla LS che va compresa ed interpretata, sia sulla qualità dell'italiano in cui va stesa la traduzione, contribuendo così all'educazione linguistica complessiva - perché questa è una delle grandi potenzialità della traduzione scritta: agisce contemporaneamente su entrambe le lingue, le culture e le enciclopedie di riferimento. $(2010,191)$

Inoltre, essa contribuisce alla formazione della CALP, ossia di quella competenza linguistico-cognitiva sofisticata che secondo Cummins (1979) è alla base del ragionamento astratto, oltre a costituire una sfida stimolante per lo studente, soprattutto se viene inserita all'interno di attività didattiche ispirate ad un approccio per compiti (cf. Mazzotta 2014). Come sintetizza efficacemente Cook, «for most contemporary language learners, translation should be a major aim and means of language learning, and a major measure of success» (2010, XV). Su questa linea, Di Martino e Di Sabato $(2015,30)$ incitano a un «full theoretical re-appraisal of translation as a pedagogical tool» rimarcando due aspetti chiave: 
(1) when learning a FL, reformulating input is a spontaneous process, and as such it should be encouraged in order to make the best use of all communication mechanisms; (2) encouraging the use of different languages and codes in the class, by capitalising on all the different means at their disposal, prepares the learners to communicate effectively in a society where extra-linguistic devices are increasingly more present and exploited. Teaching a FL and disregarding the potentialities offered by translation in all its possible forms is certainly counter-productive, especially in the specific case of English as an International Language: individuals of different mother tongues worldwide naturally compensate for their vocabulary gaps by resorting to reformulation and extra-linguistic devices. $(2015,30)$

Nonostante i diversi studi autorevoli nazionali e internazionali analizzati in questo scritto, dobbiamo ahinoi constatare che gli effetti di una riabilitazione della traduzione nella didattica quotidiana delle lingue straniere ancora non si avvertono sufficientemente: vanno dunque ancora sostenuti da ulteriori appoggi accademici.

\section{Bibliografia}

Arcaini, E. (1991). Analisi linguistica e traduzione. Bologna: Patron.

Arcaini, E. (1992). «Modelli teorici per la traduzione», in «La traduzione, Saggi e Commenti, vol. 1», Quaderni di Libri e Riviste d'Italia, 28, 15-25.

Baker, M.; Saldanha, G. (eds) (2020). Routledge Encyclopedia Of Translation Studies. 3rd ed. London: Routledge.

Balboni, P.E. (2007). «Didattica delle microlingue e uso veicolare della lingua: il ruolo della traduzione». Mazzotta, P.; Salmon, L. (a cura di), Tradurre le microlingue scientifico-professionali: Riflessioni teoriche e proposte didattiche. Torino: UTET, 49-63.

Balboni, P.E. (2010). «La traduzione nell'insegnamento delle lingue: dall'ostracismo alla riscoperta». De Giovanni, F.; Di Sabato, B. (a cura di), Tradurre in pratica: riflessioni, esperienze, testimonianze. Napoli: Edizioni Scientifiche Italiane, 179-200.

Bassnett, S. (2002). Translation Studies. 3rd ed. London: Routledge.

Bell, R.T. (1991). Translation and Translating. London: Longman.

Chesterman, A. (1997). Memes of Translation. Amsterdam: Benjamins.

Cook, G. (2010). Translation in Language Teaching: An Argument for Reassessment. Oxford: Oxford University Press.

Consiglio d'Europa (2002). Quadro comune europeo di riferimento per le lingue: apprendimento, insegnamento, valutazione. Milano: La Nuova Italia-Oxford.

Cummins, J. (1979). «Cognitive/Academic Language Proficiency, Linguistic Interdependence, the Optimum Age Question and Some Other Matters». Working Papers on Bilingualism, 19, 121-9.

Di Martino, E. (2012). «La traduzione come strumento per l'apprendimento delle lingue». Di Sabato, B.; Mazzotta, P.; Di Martino, E.; Pergola, R., Apprendere a 
tradurre. Tradurre per apprendere. La traduzione come obiettivo e strumento di apprendimento in ambito microlinguistico. Lecce: Pensa MultiMedia, 307-39.

Di Martino, E.; Di Sabato, B. (2012). «ll ruolo della traduzione nella didattica dell'inglese (e non solo) nella scuola primaria». Santipolo, M. (a cura di), Educare i bambini alla lingua inglese. Teoria e pratica nell'insegnamento dell'inglese nella scuola primaria e dell'infanzia. Lecce: Pensa MultiMedia, 319-60.

Di Martino, E.; Di Sabato, B. (2015). «Translation in SLA: A Complete Re-Appraisal». Language in Society and Professional Domains: Linguistic and Educational Issues, 5, 26-32.

Di Sabato, B. (2007). «La traduzione e l'apprendimento/insegnamento delle lingue». Studi di Glottodidattica, 1, 47-57.

Dreyfus, H.L.; Dreyfus, S.E. (1986). Mind over Machine. The Power of Human Intuition and Expertise in the Era of the Computer. Oxford: Blackwell.

González Davies, M. (2004). «Undergraduate and postgraduate translation degrees: Aims and expectations». Malmjkær, K. (ed.), Translation as an Undergraduate Degree. Amsterdam: Benjamins, 67-81. https://doi. org/10.1075/btl.59.06gon.

Göpferich, S. (2009). «Towards a Model of Translation Competence and Its Acquisition: The Longitudinal Study TransComp». Göpferich, S.; Jakobsen, A.L.; Mees, I.M. (eds), Behind the Mind: Methods, Models and Results in Translation Process Research. Copenhagen: Samfundslitteratur, 12-38.

Gouadec, D. (2007). Translation as a Profession. Amsterdam: Benjamins.

Gutt, E.-A. (2000). «Issues of Translation Research in the Inferential Paradigm of Communication». Olohan, M. (ed.), Intercultural Faultlines: Research models in Translation Studies I: Textual and Cognitive Aspects. Manchester: St. Jerome, 161-79.

Harris, B. (1977). «The Importance of Natural Translation». Working Papers in Bilingualism, 12, 96-114.

Harris, B. (1980). «How a Three-Year-Old Translates». Afendras, E.A. (ed.), Patterns of Bilingualism. Singapore: RELC, 370-93.

Harris, B.; Sherwood, B. (1978). «Translating as an Innate Skill». Gerver, D.; Sinaiko, H.W. (eds), Language Interpretation and Communication. Boston (MA): Springer, 155-70. NATO Conference Series 6. https://doi. org/10.1007/978-1-4615-9077-4_15.

Hofstadter, D.R. (1979). Gödel, Escher, Bach: An Eternal Golden Braid. New York: Basic Books.

Hofstadter, D.R. (1997). Le Ton Beau de Marot: In Praise of the Music of Language. New York: Basic Books.

Hurtado Albir, A. (1996a). «La enseñanza de la traducción directa 'general.' Objetivos de aprendizaje y metodología». Hurtado Albir, A. (ed.), La enseñanza de la traducción. Castellón: Universitat Jaume I, 31-55. https://doi. org/10.6035/estudistraduccio.1996.3.

Hurtado Albir, A. (1996b). «La cuestión del método traductor. Método, estrategia y técnica de traducción». Sendebar, 7, 39-57.

Hurtado Albir, A. (2015). «The Acquisition of Translation Competence. Competences, Tasks, and Assessment in Translator Training». Meta, 60(2), 256-80. https://doi.org/10.7202/1032857ar.

Hurtado Albir, A. (ed.) (2017). Researching Translation Competence by PACTE Group. Amsterdam: Benjamins. https://doi.org/10.1075/btl.127.

Hymes, D. (1971). On Comunicative Competence. Philadelphia: University of Pennsylvania Press. 
Kelly, D. (2005). A Handbook for Translator Trainers. Manchester: St Jerome.

Kiraly, D.C. (1995). Pathways to Translation: From Process to Pedagogy. Kent (Ohio): Kent State University Press.

Kussmaul, P. (1995). Training the Translator. Amsterdam: Benjamins.

Lefevere, A. (1975). Translating Poetry: Seven Strategies and a Blueprint. Amsterdam: Van Gorcum.

Malmkjær, K. (ed.) (1998). Translation and Language Teaching: Language Teaching and Translation. Manchester: St. Jerome.

Malmkjær, K. (ed.) (2004). Translation in Undergraduate Degree Programmes. Amsterdam: Benjamins.

Malmkjær, K. (2009). «What is Translation Competence?». Revue française de linguistique appliquée, 14, 121-34. https://doi.org/10.3917/ rfla.141.0121.

Malmkjær, K.; Windle, K. (eds) (2011). The Oxford Handbook of Translation Studies. Oxford: Oxford University Press.

Mazzotta, P. (2002). «Le direttive della Comunità Europea per l'insegnamento delle lingue». Mazzotta, P. (a cura di), Europa, lingue e istruzione primaria. Torino: UTET, 3-29.

Mazzotta, P. (2007). «Riflessioni glottodidattiche sulla traduzione delle microlingue». Montella, C.; Marchesini, G. (a cura di), I saperi del tradurre: analogie, affinità, confronti. Milano: Franco Angeli, 133-45.

Mazzotta, P. (2008). «Una proposta didattica per l'apprendimento della traduzione specializzata». De Giovanni, F.; Di Sabato, B. (a cura di), Imparare ad imparare. Imparare ad insegnare. Napoli: Edizioni Scientifiche Italiane, 115-27.

Mazzotta, P. (2014). «Lo sviluppo della competenza traduttiva attraverso l'approccio per compiti». Di Sabato, B.; Perri, A. (a cura di), I confini della traduzione. Padova: libreriauniversitaria.it, 115-26.

Millán, C.; Bartrina, F. (eds) (2012). The Routledge Handbook of Translation Studies. London: Routledge.

Ministero della Pubblica Istruzione (2007). Indicazioni per il curricolo della scuola dell'infanzia e del primo ciclo di istruzione. Roma: Ministero della Pubblica Istruzione.

Neubert, A. (1994). «Competence in Translation: A Complex Skill, How to Study and How to Teach It». Snell-Hornby, M.; Pöchhacker, F.; Kaindl, K. (eds), Translation Studies: An Interdiscipline. Amsterdam: Benjamins, 411-20. https://doi.org/10.1075/btl.2.48neu.

Nord, C. (1992). «Text Analysis in Translator Training». Dollerup, C.; Loddegaard, A. (eds), Teaching Translation and Interpreting: Training, Talent, and Experience. Amsterdam: Benjamins, 39-48.

PACTE (2000). «Acquiring Translation Competence: Hypotheses and Methodological Problems in a Research Project». Beeby, A.; Ensinger, D.; Presas, M. (eds), Investigating Translation. Amsterdam: Benjamins, 99-106. https:// doi.org/10.1075/btl.32.13pac.

PACTE (2003). «Building a Translation Competence model». Alves, F. (ed.), Triangulating Translation: Perspectives in Process Oriented Research. Amsterdam: Benjamins, 43-66. https://doi.org/10.1075/btl.45.06pac.

PACTE (2014). «First Results of PACTE Group's Experimental Research on Translation Competence Acquisition: The Acquisition of Declarative Knowledge of Translation», in Muñoz Martín, R. (ed.), «Minding Translation. Con la traducción en mente», special issue 1, MonTl: Monografías de Traducción e Interpretación, 85-115. https://doi.org/10.6035/monti.2014.ne1.2. 
PACTE (2015). «Results of PACTE's Experimental Research on the Acquisition of Translation Competence: The Acquisition of Declarative and Procedural Knowledge in Translation. The Dynamic Translation Index». Translation Spaces, 4(1), 29-53. https://doi.org/10.1075/ts.4.1.02bee.

Pierini, P. (2001). Lo sviluppo della competenza traduttiva: Orientamenti, problemi e proposte. Roma: Bulzoni.

Pym, A.; Malmkjær, K.; Gutiérrez-Colón Plana, M. (2013). Translation and Language Learning: The Role of Translation in the Teaching of Languages in the European Union. Luxembourg: Publications Office of the European Union.

Risku, H. (1998). Translatorische Kompetenz: Kognitive Grundlagen des Übersetzens als Expertentätigkeit. Tübingen: Stauffenburg.

Risku, H.; Dickinson, A.; Pircher, R. (2010). «Knowledge in Translation Practice and Translation Studies: Intellectual Capital in Modern Society». Gile, D.; Hansen, G.; Pokorn, N.K. (eds), Why Translation Studies Matters. Amsterdam: Benjamins, 83-96. https://doi.org/10.1075/btl.88.08ris.

Rothe-Neves, R. (2007). «Notes on the Concept of Translator's Competence». Quaderns: revista de traducció, 14, 125-38.

Saldanha, G.; O’Brien, S. (2013). Research Methodologies in Translation Studies. Manchester: St. Jerome.

Shreve, G.M. (1997). «Cognition and the Evolution of Translation Competence». Danks, J.H.; Shreve, G.M.; Fountain, S.B. et al. (eds), Cognitive Processes in Translation and Interpreting. Thousand Oaks: SAGE, 120-36.

Shreve, G.M. (2006). «The Deliberate Practice: Translation and Expertise». Journal of Translation Studies, 9(1), 27-42.

Titone, R. (1986). Cinque millenni di insegnamento delle lingue. Brescia: La Scuola.

Toury, G. (1984). «The Notion of 'Native Translator' and Translation Teaching». Wilss, W.; Thome, G. (eds), Translation Theory and its Implementation in the Teaching of Translating and Interpreting. Tubingen: Gunter Narr, 186-95.

Toury, G. (1995). Descriptive Translation Studies - and beyond. Amsterdam: Benjamins.

Vermeer, H.J. (1998). «Didactics of Translation». Baker, M. (ed.), Routledge Encyclopedia of Translation Studies. London: Routledge, 60-3.

Wilss, W. (1976). «Perspectives and Limitations of a Didactic Framework for the Teaching of Translation». Brislin, R.W. (ed.), Translation. New York: Gardner, 117-37.

Wilss, W. (1982). The Science of Translation: Problems and Methods. Tubingen: Gunter Narr.

Wilss, W. (1998). «Decision Making in Translation». Baker, M. (ed.), Routledge Encyclopedia of Translation Studies. London: Routledge, 57-60.

Zojer, H. (2009). «The Methodological Potential of Translation in Second Language Acquisition: Re-Evaluating Translation as a Teaching Tool». Witte, A.; Harden, T.; Ramos de Oliveira Harden, A. (eds), Translation in Second Language Learning and Teaching. Bern: Peter Lang, 31-51. 



\section{Rivista quadrimestrale}

Centro di Ricerca sulla Didattica delle Lingue, Dipartimento di Studi Linguistici e Culturali Comparati, Università Ca' Foscari Venezia

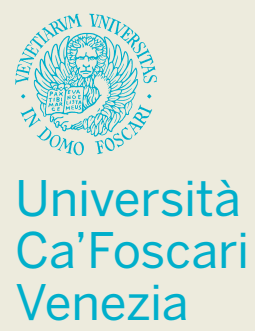

